\title{
Optimization of radiotherapy in lung cancer treatment : balance between survival and quality of life
}

Citation for published version (APA):

Pijls-Johannesma, M. (2008). Optimization of radiotherapy in lung cancer treatment : balance between survival and quality of life. [Doctoral Thesis, Maastricht University]. Datawyse / Universitaire Pers Maastricht. https://doi.org/10.26481/dis.20081209mp

Document status and date:

Published: 01/01/2008

DOI:

10.26481/dis.20081209mp

Document Version:

Publisher's PDF, also known as Version of record

\section{Please check the document version of this publication:}

- A submitted manuscript is the version of the article upon submission and before peer-review. There can be important differences between the submitted version and the official published version of record.

People interested in the research are advised to contact the author for the final version of the publication, or visit the DOI to the publisher's website.

- The final author version and the galley proof are versions of the publication after peer review.

- The final published version features the final layout of the paper including the volume, issue and page numbers.

Link to publication

\footnotetext{
General rights rights.

- You may freely distribute the URL identifying the publication in the public portal. please follow below link for the End User Agreement:

www.umlib.nl/taverne-license

Take down policy

If you believe that this document breaches copyright please contact us at:

repository@maastrichtuniversity.nl

providing details and we will investigate your claim.
}

Copyright and moral rights for the publications made accessible in the public portal are retained by the authors and/or other copyright owners and it is a condition of accessing publications that users recognise and abide by the legal requirements associated with these

- Users may download and print one copy of any publication from the public portal for the purpose of private study or research.

- You may not further distribute the material or use it for any profit-making activity or commercial gain

If the publication is distributed under the terms of Article $25 \mathrm{fa}$ of the Dutch Copyright Act, indicated by the "Taverne" license above, 


\section{Optimization of radiotherapy in lung cancer treatment:}

Balance between survival and Quality of Life 
ISBN 9789052787879

(c) Copyright M. Pijls-Johannesma, Maastricht 2008

Druk: Datawyse | Universitaire Pers Maastricht

Cover Illustrations: Mirthe \& Lotte Pijls 


\title{
Optimization of radiotherapy in lung cancer treatment: \\ Balance between survival and Quality of Life
}

\author{
Proefschrift \\ ter verkrijging van de graad van doctor \\ aan de Universiteit Maastricht, \\ op gezag van de Rector Magnificus \\ Prof. mr. G.P.M.F. Mols, \\ volgens het besluit van het College van Decanen, \\ in het openbaar te verdedigen, \\ op dinsdag 9 december 2008 om 16.00 uur \\ door
}

Madelon Pijls-Johannesma

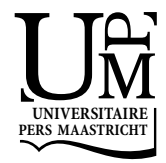




\section{Promotor}

Prof. dr. Ph. Lambin

\section{Copromotores}

Dr. D.K.M. De Ruysscher

Dr. L.J. Boersma

\section{Beoordelingscommissie}

Prof. dr. E.F. Wouters (voorzitter)

Dr. A.M. Dingemans

Prof. dr. J.A. Langendijk (UMCG)

Prof. dr. Y. Lievens (UH Leuven)

Prof. dr. J.L. Severens 


\section{CONTENTS}

Chapter 1 Introduction and outline of the thesis

Chapter 2 Timing of chest radiotherapy in patients with limited disease small cell lung cancer: A systematic review and meta-analysis of randomised controlled trials

Cancer Treatment Reviews (2007), 33: 461- 473

Chapter 3 Time between the first day of chemotherapy and the last lay of chest radiation Is the most important predictor of survival in limited-disease small-cell lung cancer Journal of Clinical Oncology (2007), 24:1057-1063

Chapter 4 Health related quality of life assessment instruments:

A prospective study on preference and acceptability among cancer patients referred for radiotherapy

European Journal of Cancer (2005), 41: 2250-2256

Chapter 5 Impact of fatigue on overall quality of life in lung and breast cancer patients selected for high-dose radiotherapy Annals of Oncology (2007), 18: 940-944

Chapter 6 High dose radiotherapy or concurrent chemo-radiation in lung cancer patients only induces a temporary, reversible decline in QoL.

Submitted

Chapter 7 Particle therapy in lung cancer: were do we stand?

Cancer Treatment Reviews (2008), 34:259-67

Journal of Clinical Oncology (2007), 25:956-970

Radiotherapy and Oncology (2007), 83:110-122

Chapter 8 Cost-effectiveness of particle therapy: current evidence and future needs

Radiotherapy and Oncology (2008), in press

Chapter 9 General Discussion and Future Perspectives 
Samenvatting

Dankwoord

Curriculum Vitae

175

Publications

Notes 


\section{ABBREVIATIONS}

\begin{tabular}{|c|c|c|c|}
\hline AUC & Area under the curve & MEC & Medical Ethical Committee \\
\hline BED & Biological Effective Doses & MFI & $\begin{array}{l}\text { Multidimensional Fatigue } \\
\text { Inventory }\end{array}$ \\
\hline BMI & Body mass index & ML & Maximum Likelihood \\
\hline СBA & Cost-benefit analysis & MQ & Maastricht Questionnaire \\
\hline CEA & Cost-effectiveness analysis & NS & Not significant \\
\hline CEAC & $\begin{array}{l}\text { Cost-effectiveness acceptability } \\
\text { curves }\end{array}$ & NSCLC & Non-small cell lung cancer \\
\hline CGE & Cobalt Gray Equivalent & OR & Odds ratio \\
\hline CHART & $\begin{array}{l}\text { Continuous hyperfractionated } \\
\text { accelerated radiotherapy }\end{array}$ & os & Overall survival \\
\hline $\mathrm{Cl}$ & Confidence Interval & OTT & Overall treatment time \\
\hline C-ion & Carbon-ion & $\mathrm{PCl}$ & Prophylactic cranial irradiation \\
\hline CMA & Cost-minimization analysis & PET & Positron emission tomography \\
\hline CRT & Chest radiotherapy & PFS & Piper Fatigue Scale \\
\hline css & Cause-specific survival & PRO & Patient-reported outcome \\
\hline CT & Chemotherapy & PS & Performance status \\
\hline Ст & Computer tomography & PT & Particle therapy \\
\hline СтC & Common Toxicity Criteria & QALY & Quality adjusted life years \\
\hline CTR & Concurrent chemo-radiation & QLQ-C30 & $\begin{array}{l}\text { Quality of Life Questionnaire } \\
\text { Core } 30\end{array}$ \\
\hline CUA & Cost-utility analysis & QoL & Quality of Life \\
\hline E/L & Early /late & REML & Restricted Maximum Likelihood \\
\hline EORTC & $\begin{array}{l}\text { European Organization for Re- } \\
\text { search and Treatment of Cancer }\end{array}$ & $\mathbf{R R}$ & relative risk \\
\hline EQ-5D & Euroqol 5D & RSCL & Rotterdam Symptom Checklist \\
\hline EVPI & $\begin{array}{l}\text { Expected value of perfect infor- } \\
\text { mation }\end{array}$ & RT & Radiotherapy \\
\hline FACT & $\begin{array}{l}\text { Functional Assessment of Cancer } \\
\text { Therapy }\end{array}$ & rRTOG & $\begin{array}{l}\text { Radiation Therapy Oncology } \\
\text { Group }\end{array}$ \\
\hline FDG & ${ }^{18} \mathrm{~F}$-deoxyglucose & SBRT & Stereotactic body radiotherapy \\
\hline HR & Hazard ratio & SCLC & Small-cell lung cancer \\
\hline HRQoL & Health related quality of life & SD & Standard deviation \\
\hline ICER & $\begin{array}{l}\text { Incremental cost-effectiveness } \\
\text { ratio }\end{array}$ & SE & Standard error \\
\hline IMRT & $\begin{array}{l}\text { Intensity Modulated Radio Ther- } \\
\text { apy }\end{array}$ & SER & $\begin{array}{l}\text { Start of any treatment until the } \\
\text { End of Radiotherapy }\end{array}$ \\
\hline KPS & Karnofsky performance score & SRC & $\begin{array}{l}\text { Standardized regression coeffi- } \\
\text { cient }\end{array}$ \\
\hline KvL & Kwaliteit van Leven & SRT & Stereotactic radiotherapy \\
\hline LC & Local control & sWOG & Southwest Oncology Group \\
\hline LD-SCLC & Limited disease-SCLC & VAS & Visual Analogue Scale \\
\hline LYG & Life year gained & WHO & World Health Organization \\
\hline
\end{tabular}





\section{CHAPTER}

Introduction and outline of the thesis 



\section{INTRODUCTION AND OUTLINE OF THE THESIS}

\section{Background}

Overall, 1.7 million cancer deaths are estimated in Europe in $2006^{1}$, with lung cancer being one of the most lethal malignancies, with one-fifth of the total number of cancer deaths. Although the incidence of lung cancer for men has decreased by one third between 1989 and 2003, for women the incidence has increased with $80 \%$ in the same period. This can be explained by increasingly similar smoking habits of men and women which have resulted in a convergence of lung cancer incidence between men and women. The majority of lung cancers (80\%) are non small cell lung cancer (NSCLC), whereas the rest is small cell lung cancer (SCLC).

Radiotherapy (RT) is one of the most important curative and palliative treatment modalities for both NSCLC and SCLC. RT has made significant progress in the past years, due to more advanced technologies, better staging, insights in radiation (molecular) biology and combined modality approaches. It has consistently been shown that increased local tumor control by concurrent chemo-radiation (CTR) is translated in increased survival ${ }^{2,3}$. Moreover, increasing the radiation dose leads to an increased local tumor control ${ }^{4,5}$. Fletcher ${ }^{4}$ estimated that radiation doses of $60 \mathrm{~Gy}$ in 30 fractions of $2 \mathrm{~Gy}$ are effective in controlling post-operative microscopic disease, but up to 100 Gy might be necessary to sterilize the tumor as a whole. Theoretically, a dose of $>84 \mathrm{~Gy}$ is necessary to obtain a chance for a definite local control in small tumors of $50 \%{ }^{6}$. However, delivery of high-dose RT requires restrictions of the total tumor dose and tumor volume in order to minimize the risk of complications in the surrounding normal tissues. Higher doses to the normal tissue will cause more treatment related side effects which are expected to result in a reduction of a patients' Quality of Life (QoL). Since overall survival is improving, QoL is increasingly being recognized as a very important endpoint to evaluate the treatment effect. Worldwide, many research groups are investigating RT or CTR for lung cancer, with the aim to improve survival with preservation of a patients' QoL. At present, this goal has still not been achieved.

This thesis therefore examines curative radiation treatment for lung cancer in order to get insight in the balance between the beneficial and adverse effects of intensive radiotherapy both with respect to survival and QoL. The ultimate goal is to enable adequate decision making. For this latter, the role of new technologies in lung cancer is also investigated.

The thesis is divided in three parts (figure 1).

1. Determination of radiation treatment factors that improve survival.

2. QoL of lung cancer patients before, during and after intensive radiotherapy.

3. Decision making on new innovative technologies: effectiveness, QoL, and costs. 

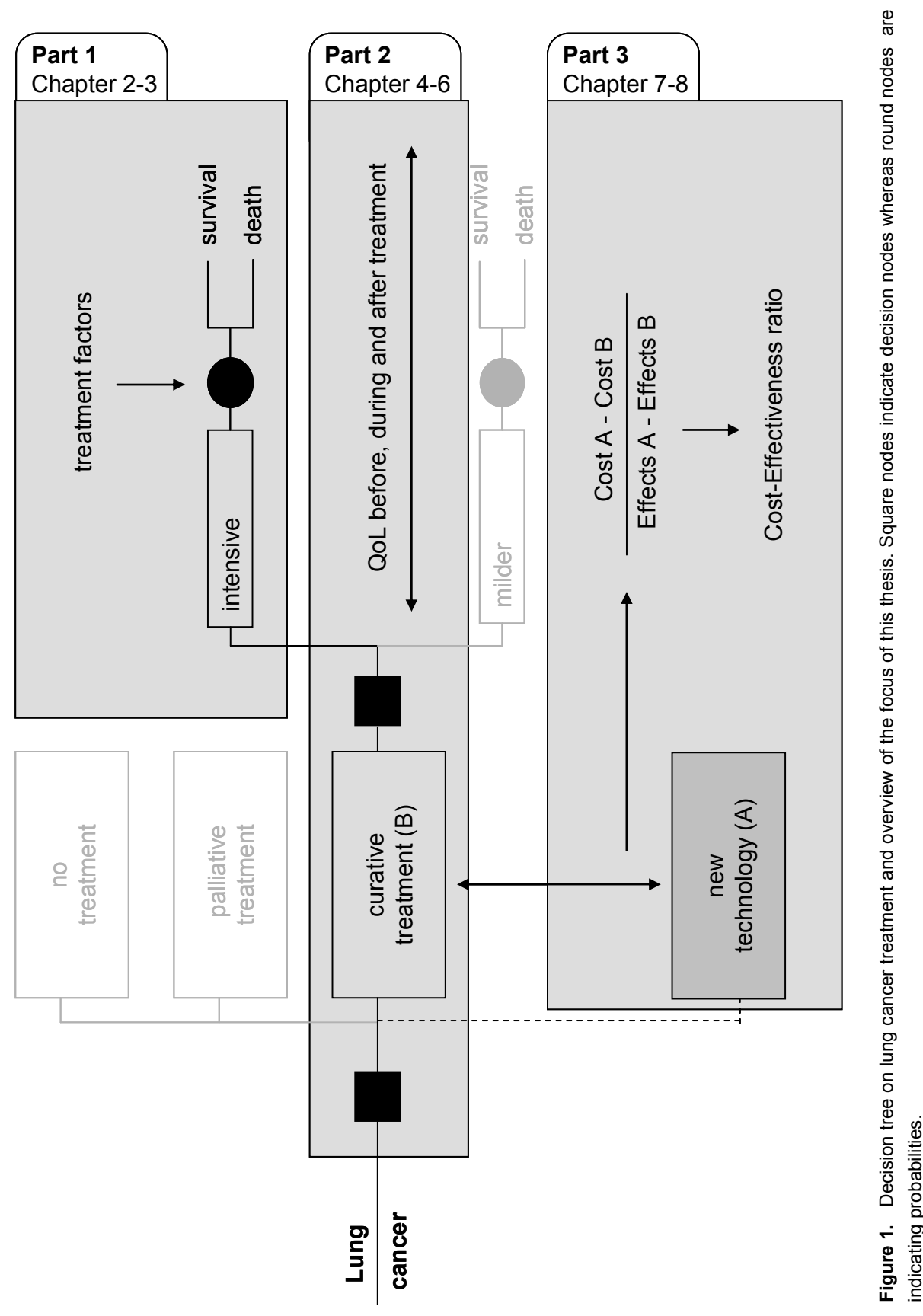


\section{PART 1}

\section{Determination of radiation treatment factors that improve survival}

\section{Patient selection and survival}

The standard treatment for stage I and II operable NSCLC is surgical resection followed by adjuvant chemotherapy in stage II, whereas the standard of care for medically inoperable stage I-II NSCLC is radiotherapy. Stage III NSCLC is treated with chemo-radiation, with surgery in selected cases ${ }^{7}$. Outcomes after conventional radiotherapy in patients with inoperable stage I NSCLC are modest, with a literature review reporting 3 - and 5-year disease-specific survivals of $39 \pm 10 \%$ and $25 \pm 9 \%$, and overall survivals around $34 \pm 9 \%$ and $21 \pm 8 \%$, respectively ${ }^{8}$, whereas after surgery the 5 -year overall survival is around $67 \%$ for stage IA tumors and $57 \%$ for stage IB tumors ${ }^{9}$. In view of the relatively poor survival rates after radiotherapy, it would be of highly interest to get insight in the benefits from new RT technologies on outcome of lung cancer treatment, and to examine the clinical evidence from new technologies. In the case of lung cancer, stereotactic body radiotherapy and particle therapy would be of particular interest.

Limited disease-SCLC (LD-SCLC), comprises only one third of the total SCLC patients ${ }^{10,11}$. Without treatment, tumor progression in patients with SCLC is rapid, with a median survival of 2 to 4 months. Chemotherapy has improved the median survival time substantially, but long-term survival remains rare after chemotherapy alone ${ }^{12}$. Two meta-analyses in LD-SCLC ${ }^{2,3}$ have shown an improvement of $5.4 \%$,in absolute survival at 2-years and 3-years, in patients who received chest irradiation and chemotherapy versus those receiving chemotherapy alone. The 5-year survival rate remains disappointingly low at $10-15 \%^{2}$. Although evidence for a significant survival benefit of adding chest radiotherapy was provided by the meta-analyses, no conclusions could be drawn regarding the optimal timing and sequencing of chemotherapy and radiation ${ }^{13,14}$. Establishing the most effective way of combining chest radiotherapy with chemotherapy and to identify time factors for patients with limited-stage small cell lung cancer could help to eventually improve long-term survival.

For stage IV NSCLC and SCLC -extensive disease, no curative treatment options are available. These patients are usually treated with palliative chemotherapy or sometimes palliative radiotherapy and with best supportive care. 
As described above, the treatment usually consists of surgery, radiotherapy, or chemotherapy, or a combination of these treatment modalities ${ }^{13,14}$. Next to it, targeted therapies are highly under development such as Avastin ${ }^{\mathrm{TM}}$ (bevacizumab) which enhances the efficacy of front-line chemotherapy ${ }^{15}$. The choice for a certain therapy is based on the balance between the expected probability of tumor control and expected probability and severity of toxicity and estimated ability of the patient to cope with toxicities. The probability of tumor control and toxicity in turn depend on the general health status of the patients (patient factors), the tumor histology, and the stage of the disease (tumor factors) Finally, apart from guidelines, also physician and patients preferences will play also a role in selecting the final treatment of choice.

\section{Prognostic and predictive factors}

Prognostic factors are defined as those at the time of diagnosis that are able to give information on clinical outcome. Predictive factors are those that are able to provide information useful in the selection of patients likely to respond to therapies. The predictability of population survival outcomes may be of limited usefulness to clinicians due to the heterogeneity of patients comprising the overall population.

Prognostic and predictive factors are, thus, used to divide the population into subgroups in order to realize the benefits of stratification ${ }^{16}$, including improved medical decision making ${ }^{17}$, improved personal decision making beyond the treatment decision, more appropriate research design and analysis, and more appropriate health policy development ${ }^{18}$.

Clinical factors such as the extent of disease, tumor volume, weight loss, and the performance status have been identified as prognostic factors and have a significant impact on survival ${ }^{19,20}$. However, several studies ${ }^{21-24}$ found that global quality of life was to be the strongest prognostic factor for survival. Remarkably, if pre-treatment QoL is taken into account, it appears that clinical factors like performance status are no independent predictors of survival anymore. It would therefore be important to identify determinants that influence pre-treatment QoL in order to anticipate on the prognosis of a specific patient and to adequately consider the most optimal treatment. 


\section{PART 2}

\section{QoL of lung cancer patients before, during and after intensive radiotherapy}

\section{Quality of Life}

Quality of life (QoL) in lung cancer patients is an important treatment outcome in addition to length of survival. Patients' assessments of their QoL are shown to differ from their physicians ${ }^{25}$. Getting insight in patients' own perspective on QoL can add important information in decision-making for the most adequate treatment strategy. Acknowledgements of the need to capture patient's perspectives of the impact of illness and health care interventions has grown rapidly over the last decades ${ }^{26}$.

Therefore, the evaluation of QoL in treated patients is nowadays getting more important within medical practice. Also the shift from acute to chronic diseases and the process of optimizing medical care demands a growing concern for the QoL of patients. Long term adverse consequences of cancer treatment are limited reported, due to the high mortality rate which has made it difficult to assess treatment related toxicity ${ }^{27}$. The most reported complication after surgery is chronic pain, known as postthoracotomy pain syndrome ${ }^{28}$, whereas many chemotherapy agents cause cardiopulmonary or other toxicities ${ }^{27}$. Frequently reported adverse effects after radiotherapy are pneumonitis and reduction of pulmonary function. Radiation pneumonits (SWOG, grade $\geq 2$ ) was reported in 15 to $20 \%$ of the patients after receiving externalbeam radiotherapy ${ }^{29,30}$.

There is a general agreement that patient-reported outcome (PRO) assessment in health care should precede from a strong conceptual basis, with a-priori definitions describing clearly how to be measured and how to be accomplished ${ }^{26,31}$.

The usual method of collecting QoL information is by self-assessment questionnaires. During the last two decades, a number of these questionnaires have been developed ${ }^{32-36}$. Over the last decade, QoL measures have been routinely incorporated in oncological clinical trials. QoL is generally considered as a multidimensional construct, which includes several key dimensions ${ }^{37}$.

Drummond et al. ${ }^{38}$ describe three main types of QoL scales:

1. (disease) specific measures

2. general health profiles

3. preference-based measures.

The advantage of disease-specific questionnaires is that they are likely to be responsive to changes in a patients condition and that they are likely to be seen as most relevant to patients and physicians and therefore more accepted ${ }^{38}$. Therefore most implications of this type of scale are seen in clinical studies. General health profiles 
(generic) are comprehensive measures of QoL. However, from an economist's viewpoint there are some disadvantages using this type of scale. The scoring of general health profiles is not based on preferences of individuals for various possible outcomes, by which it is not clear that higher scores are necessarily associated with outcomes that are more preferred. In addition, it is not possible to calculate a QALY with the score of this scale. On the other hand, scores of preference-based (or utility) measures do have these possibilities and are therefore extensively used in cost-utility analysis.

Generic questionnaires can be applied to a variety of health conditions, impairments and populations. Examples are the Medical Outcomes Study form health surveys, SF-36 and MOS-24 ${ }^{39,40}$, and the Euroqol 5D(EQ-5D) ${ }^{41}$. These generic instruments can be useful for comparing the QoL of a group of physically impaired persons with that of a healthy population. However, as they contain little or no relevance for specific diseases, questionnaires have been developed that are tailored to patients suffering from a particular disease.

Examples of disease specific questionnaires are the MOS-HIV ${ }^{42}$ for those with HIVIAIDS and the EORTC-QLQ-C $30^{32}$ for cancer patients. Disease specific questionnaires are expected to be more sensitive to clinically significant changes.

So far, evaluations have mainly focused on psychometric properties of such QoL scales and not on the patient's preferences and acceptability of these scales. Preference and acceptability are nevertheless important issues for several reasons. First, the assessment of QoL in cancer patients is often hampered by the incompletion of scales, a phenomenon which can be caused by questions that are unclear to patients or questions that are considered as offensive to patients. Second, if these instruments are not filled out in the correct manner, this may lead to incorrect conclusions. Third, in the setting of a busy outpatient clinic with limited staff, QoL instruments must be self-instructive, easily understandable and reasonably convenient for the patient in terms of time and effort.

Fatigue (defined as a general feeling of debilitating tiredness or loss of energy) is one of the most common quality of life related symptoms in cancer today ${ }^{43-48}$. The occurrence of fatigue varies across tumor sites ${ }^{46,49,50}$ and treatment stage ${ }^{46,49,51-54}$. Lung cancer patients undergoing radiotherapy had the highest levels of fatigue relative to patients of other tumor types ${ }^{46}$. For patients and oncologists, improving the quality of life of cancer patients requires a heightened awareness of fatigue, a better understanding of its impact, and improved communication and familiarity with interventions that can reduce its debilitating effects ${ }^{55}$. However, despite previous surveys on patients' subjective and conscious valuation of the impact of fatigue on daily life, few if any studies so far have made an attempt to quantify the impact of fatigue, relatively to other QoL domains, on overall QoL in cancer patients prior to high-dose radiotherapy. 
In this thesis QoL is examined for curative radiation treatment for lung cancer, in order to get insight in the balance between the beneficial and adverse effects of intensive radiotherapy both with respect to survival and QoL. To be able to pronounce upon the mechanism of the complex multidimensional construct of QoL in lung cancer it is necessary to have knowledge about adequate QoL assessment. Next to it, as described earlier, assessment of pre-treatment QoL is important to identify which factors are influencing the overall QoL in cancer patients preceding radiotherapy. In addition, pre-treatment QoL could be useful to identify subgroups of patients before treatment in order to enable adequate decision making between different treatment options. Finally, for the aim of this thesis, the evolution of QoL in patients with lung cancer, selected for intensive RT must become noticeable.

\section{PART 3}

Decision making on new innovative technologies: effectiveness, QoL and costs

In this era of fast growing technologies, which are mostly accompanied by higher costs, an increasingly important issue in the field of radiation oncology is how the costs of treatments relate to their effects. Typically, the extra cost of a new intervention (compared to the standard treatment) must be balanced with its additional gain in clinical effect (i.e., better local control and/or survival, decreased morbidity/better QoL) by means of economic evaluations. From Table 1 it will be clear that only evaluations investigating both costs and effects, with a comparison between alternatives, can claim to provide full economic evaluation.

Table 1: Requirements needed for different types of economic evaluations.

\begin{tabular}{|c|c|c|c|c|}
\hline \multirow{6}{*}{$\begin{array}{l}\text { Is there a comparison between } \\
\text { two alternatives? }\end{array}$} & & \multicolumn{3}{|c|}{ Are both costs (input) and effects (output) examined? } \\
\hline & & \multicolumn{2}{|c|}{ no } & \multirow[t]{2}{*}{ yes } \\
\hline & & Only effects & Only costs & \\
\hline & yes & Outcome & & Cost-outcome description \\
\hline & & description & description & \\
\hline & no & Efficacy or Effectiveness & Cost analysis & Full economic evaluation* \\
\hline
\end{tabular}

Effectiveness can be measured using various measures of outcome, such as life years gained, percentage reduction in blood pressure or event-free days. In a costeffectiveness analysis (CEA) the incremental effectiveness of an intervention is quantified and compared with its incremental costs. A cost-utility analysis (CUA) is rather similar to a CEA, but in a CUA the measure of effectiveness is quality adjusted life years (QALY). QALYs are a combined measure of life expectancy and quality of life. QoL is expressed in a utility score, ranging from $0-1$, where 0 represents death and 1 
represent perfect health ${ }^{38}$. The measure of utilities is due to preference-based scales.

\section{Particle therapy: introduction of a new innovative technology}

A major problem in the treatment of lung cancer remains local tumor control ${ }^{56,57}$. Therefore, new ways to deliver radiotherapy beyond photons $s^{8,9}$ have been sought for, including protons ${ }^{58,59}$ and carbon ions ${ }^{60-62}$. Because of their superior dosedistribution, and for $\mathrm{C}$-ions also their biological characteristics, a therapeutic gain can be expected with charged particles ${ }^{63-74}$. Accordingly, particle therapy should help to minimize the extent and severity of pulmonary injury. This could benefit patients with localized lung cancer and severe underlying pulmonary disease, but in addition it could permit an increased dose to the primary tumor, thereby improving the local tumor control probability. In order to limit the (escalation in) health care expenses, new techniques must prove that additional higher costs are worthwhile, thus costeffective.

This puts the introduction of (expensive) new irradiation treatments such as particle therapy in a difficult situation: firm data on effectiveness can only be obtained after the implementation and clinical evaluation of the treatment, which in the case of radiotherapy often implies that important capital investments must be made. Yet data on effectiveness will only become available years after the necessary investment in equipment, buildings and highly qualified personnel.

\section{Cost-effectiveness}

As an example of a current radiotherapy treatment option in lung cancer, Lievens et al ${ }^{75}$ investigated, using a Markov model, the cost-effectiveness and cost-utility of continuous hyperfractionated and accelerated radiotherapy (CHART). CHART appeared to be 1.5 more expensive than conventional external radiotherapy, due to more fractions, more hospitalization, etc. However, the incremental societal cost of $€ 2300$ at 4 years for CHART compared to conventional high-dose radiotherapy, translated in a cost-effectiveness ratio of $€ 9164$ per life-year gained and a cost-utility ratio of $€ 11,576 /$ per quality adjusted life year (QALY). Taking into account a ceiling ratio of $€ 20.000$, CHART could be considered as a cost-effective treatment option.

As mentioned, future radiotherapy treatment options like particle therapy (PT) seem due to physical and biological advantages promising. However, as this new treatment modality is relatively expensive as compared to conventional radiotherapy with photons, the gain in effects must be worthwhile with respect to the additional costs. The cost-effectiveness of PT is still under investigation. 


\section{Objectives and outline of the thesis}

In this thesis the curative radiotherapy treatment of lung cancer is examined. In order to get insight in the most optimized treatment option for a (sub) group of lung cancer patients, we searched for potential factors which influence survival as well as factors that have an impact on patients QoL. To obtain this goal we proceeded from two perspectives. The first perspective is mainly focused on improvement of survival. Different treatment factors have been examined, like the overall treatment time, and the timing of radiotherapy delivery in the modality treatment with chemotherapy to investigate which factors in the radiation treatment could contribute to improved survival. In the second perspective, i.e. the patients' experience, the focus has been pointed towards quality of life issues. With help from self-reported questionnaires insight has been gained on patients' preferences regarding QoL measurements. Furthermore, factors are investigated that may have a high impact on the overall QoL of lung cancer patients before, during, and after treatment with radiotherapy.

Finally, the impact of a new radiotherapy modality, particle therapy, on survival and QoL in the treatment of lung cancer is examined based on literature. As in this era for all new treatment modalities it has to be proven that the extra investment costs are worthwhile with respect to the expected gain, also the cost-effectiveness of particle therapy is taken into account.

The main aim of this thesis is to get insight in the most optimized radiotherapy treatment for different groups of lung cancer patients, useful in medical decision making. To obtain this goal the following research questions were described:

1. What is the most effective way of combining chest radiotherapy with chemotherapy for patients with small cell lung cancer in order to improve long-term survival?

2. What is the best way to asses QoL in patients with lung cancer?

3. Which factors have the highest impact on the QoL in lung cancer patients?

4. What is the impact of high dose radiotherapy on QoL in lung cancer patients?

The first chapters are dedicated to treatment factors that may have influence on the long-term survival. In Chapter 2 we investigate which is the most effective way of combining chest radiotherapy with chemotherapy for patients with Limited DiseaseSCLC in order to improve long-term survival. Following the results of the research performed in Chapter 2, in Chapter 3 we analyze the time factors for combined chemotherapy and radiotherapy in more detail, in order to obtain long-term survival of patients with LD-SCLC.

In the next three chapters several QoL issues are discussed regarding lung cancer patients treated with radiotherapy. The aim of the study described in Chapter $\mathbf{4}$ is to determine the preferences for and acceptability of existing Quality of Life (QoL) ques- 
tionnaires among cancer patients. Based on criteria like the percentage of missing values, difficult items and the preferences for the answer options, generally used questionnaires are examined.

Using a patient preferred assessment tool, in Chapter $\mathbf{5}$ we evaluate the relative impact of different QoL domains/subscales on overall QoL in lung cancer patients preceding radiotherapy. Next to it we also analyze the evolution of QoL in patients with lung cancer selected for curative radiotherapy or chemo-radiation. Since we hypothesized that esophageal toxicity may have a considerable impact on the QoL of the selected study population, we besides study the relationship between QoL and esophagus toxicity. These results are described in Chapter 6.

The final two chapters are dedicated to future possibilities of new radiotherapy technologies and the impact on lung cancer treatment regarding survival and QoL. Since from a theoretical point of view, charged particles should lead to superior results compared to photons, in Chapter 7 we searched for clinical evidence that protons or C-ions are really beneficial to patients with lung cancer. Finally, Chapter 8 focuses on different costing issues related to particle therapy in order to detect future needs and recommend a strategy that allows for the evidence-based determination of the cost-effectiveness of particle therapy as compared to conventional radiotherapy. 


\section{REFERENCES}

1. Ferlay J, Autier P, Boniol M, et al: Estimates of the cancer incidence and mortality in Europe in 2006. Annals of oncology 18:581-92, 2007

2. Pignon JP, Arriagada R, Ihde DC, et al: A meta-analysis of thoracic radiotherapy for small-cell lung cancer. N Engl J Med 327:1618-24, 1992

3. Warde P, Payne D: Does thoracic irradiation improve survival and local control in limited-stage smallcell carcinoma of the lung? A meta-analysis. J Clin Oncol 10:890-5, 1992

4. Fletcher $\mathrm{GH}$ : Clinical dose response curves of human malignant epithelial tumours. Br J Radiol 46:151, 1973

5. Perez CA, Pajak TF, Rubin P, et al: Long-term observations of the patterns of failure in patients with unresectable non-oat cell carcinoma of the lung treated with definitive radiotherapy. Report by the Radiation Therapy Oncology Group. Cancer 59:1874-81, 1987

6. Martel MK, Ten Haken RK, Hazuka MB, et al: Estimation of tumor control probability model parameters from 3-D dose distributions of non-small cell lung cancer patients. Lung Cancer 24:31-7, 1999

7. SIGN: Management of patients with lung cancer: A national clinical guidline, in NHS (ed): Quality Improvement Scotland, 2005

8. Qiao X, Tullgren O, Lax I, et al: The role of radiotherapy in treatment of stage I non-small cell lung cancer. Lung Cancer 41:1-11, 2003

9. Mountain CF: Revisions in the International System for Staging Lung Cancer. Chest 111:1710-7, 1997

10. Bunn PA, Jr., Carney DN: Overview of chemotherapy for small cell lung cancer. Semin Oncol 24:S769-S7-74, 1997

11. Stupp R, Monnerat C, Turrisi AT, 3rd, et al: Small cell lung cancer: state of the art and future perspectives. Lung Cancer 45:105-17, 2004

12. Kelly K: New chemotherapy agents for small cell lung cancer. Chest 117:156S-162S, 2000

13. Kumar $\mathrm{P}$ : The role of thoracic radiotherapy in the management of limited-stage small cell lung cancer: past, present, and future. Chest 112:259S-265S, 1997

14. De Ruysscher D, Vansteenkiste J: Chest radiotherapy in limited-stage small cell lung cancer: facts, questions, prospects. Radiother Oncol 55:1-9, 2000

15. Reck M, Crino L: Advances in anti-VEGF and anti-EGFR therapy for advanced non-small cell lung cancer. Lung Cancer, 2008

16. Feinstein AR: Clinical biostatistics. XIV. The purposes of prognostic stratification. Clin Pharmacol Ther 13:285-97, 1972

17. Brundage MD, Feldman-Stewart D, Cosby R, et al: Phase I study of a decision aid for patients with locally advanced non-small-cell lung cancer. J Clin Oncol.19:1326-35, 2001

18. Brundage MD, Davies D, Mackillop WJ: Prognostic factors in non-small cell lung cancer: a decade of progress. Chest 122:1037-57, 2002

19. Dehing-Oberije $\mathrm{C}$, De Ruysscher $\mathrm{D}$, van der Weide $\mathrm{H}$, et al: Tumor volume combined with number of positive lymph node stations is a more important prognostic factor than TNM stage for survival of nonsmall-cell lung cancer patients treated with (chemo)radiotherapy. Int J Radiat Oncol Biol Phys 70:103944, 2008

20. Blanchon F, Grivaux M, Asselain B, et al: 4-year mortality in patients with non-small-cell lung cancer: development and validation of a prognostic index. Lancet Oncol 7:829-36, 2006

21. Montazeri A, Milroy R, Hole D, et al: Quality of life in lung cancer patients: as an important prognostic factor. Lung Cancer 31:233-40, 2001

22. Brown GC, Brown MM, Sharma S, et al: Incremental cost-effectiveness of laser therapy for visual loss secondary to branch retinal vein occlusion. Ophthalmic Epidemiol 9:1-10, 2002

23. Montazeri A, Gillis CR, McEwen J: Quality of life in patients with lung cancer: a review of literature from 1970 to 1995 . Chest $113: 467-81,1998$ 
24. Langendijk $\mathrm{H}$, Aaronson NK, de Jong JM, et al: The prognostic impact of quality of life assessed with the EORTC QLQ-C30 in inoperable non-small cell lung carcinoma treated with radiotherapy. Radiother Oncol 55:19-25, 2000

25. Stephens RJ, Hopwood P, Girling DJ, et al: Randomized trials with quality of life endpoints: are doctors' ratings of patients' physical symptoms interchangeable with patients' self-ratings? Qual Life Res 6:22536, 1997

26. Rothman ML, Beltran P, Cappelleri JC, et al: Patient-reported outcomes: conceptual issues. Value Health 10 Suppl 2:S66-75, 2007

27. Sugimura H, Yang P: Long-term survivorship in lung cancer: a review. Chest 129:1088-97, 2006

28. Sarna L, Evangelista L, Tashkin D, et al: Impact of respiratory symptoms and pulmonary function on quality of life of long-term survivors of non-small cell lung cancer. Chest 125:439-45, 2004

29. Hernando ML, Marks LB, Bentel GC, et al: Radiation-induced pulmonary toxicity: a dose-volume histogram analysis in 201 patients with lung cancer. Int J Radiat Oncol Biol Phys 51:650-9, 2001

30. Rancati T, Ceresoli GL, Gagliardi G, et al: Factors predicting radiation pneumonitis in lung cancer patients: a retrospective study. Radiother Oncol 67:275-83, 2003

31. Revicki DA, Erickson PA, Sloan JA, et al: Interpreting and reporting results based on patient-reported outcomes. Value Health 10 Suppl 2:S116-24, 2007

32. Aaronson NK, Ahmedzai S, Bergman B, et al: The European Organization for Research and Treatment of Cancer QLQ-C30: a quality-of-life instrument for use in international clinical trials in oncology. J Natl Cancer Inst 85:365-76, 1993

33. Cella DF, Bonomi AE, Lloyd SR, et al: Reliability and validity of the Functional Assessment of Cancer Therapy-Lung (FACT-L) quality of life instrument. Lung Cancer 12:199-220, 1995

34. Ganz PA, Schag CA, Lee JJ, et al: The CARES: a generic measure of health-related quality of life for patients with cancer. Qual Life Res 1:19-29, 1992

35. de Haes JC, van Knippenberg FC, Neijt JP: Measuring psychological and physical distress in cancer patients: structure and application of the Rotterdam Symptom Checklist. Br J Cancer 62:1034-8, 1990

36. Schipper $\mathrm{H}$, Clinch J, McMurray A, et al: Measuring the quality of life of cancer patients: the Functional Living Index-Cancer: development and validation. Journal of Clinical Oncology 2:472-483, 1984

37. Velikova G, Stark D, Selby P: Quality of life instruments in oncology. Eur J Cancer 35:1571-80, 1999

38. Drummond M, Sculpher M, Torrance G, et al: Methods for the Economic Evaluation of Health Care Programmes (ed third). Oxford, Oxford medical publications, 2005

39. Stewart AL, Hays RD, Ware JE, Jr.: The MOS short-form general health survey. Reliability and validity in a patient population. Med Care 26:724-35, 1988

40. Ware JE, Jr., Sherbourne CD: The MOS 36-item short-form health survey (SF-36). I. Conceptual framework and item selection. Med Care 30:473-83, 1992

41. EuroQol-Group: EuroQol--a new facility for the measurement of health-related quality of life. Health Policy 16:199-208, 1990

42. Wu AW, Rubin HR, Mathews WC, et al: A health status questionnaire using 30 items from the Medical Outcomes Study. Preliminary validation in persons with early HIV infection. Med Care 29:786-98, 1991

43. Curt GA: Fatigue in cancer. Bmj 322:1560, 2001

44. Morrow GR, Shelke AR, Roscoe JA, et al: Management of cancer-related fatigue. Cancer Invest 23:229-39, 2005

45. Donovan KA, Jacobsen PB, Andrykowski MA, et al: Course of fatigue in women receiving chemotherapy and/or radiotherapy for early stage breast cancer. J Pain Symptom Manage 28:373-80, 2004

46. Smets EM, Visser MR, Willems-Groot AF, et al: Fatigue and radiotherapy: (A) experience in patients undergoing treatment. Br J Cancer 78:899-906, 1998

47. Richardson A: Fatigue in cancer patients: a review of the literature. Eur J Cancer Care (Engl) 4:20-32, 1995

48. Winningham ML, Nail LM, Burke MB, et al: Fatigue and the cancer experience: the state of the knowledge. Oncol Nurs Forum 21:23-36, 1994 
49. Monga U, Kerrigan AJ, Thornby J, et al: Prospective study of fatigue in localized prostate cancer patients undergoing radiotherapy. Radiat Oncol Investig 7:178-85, 1999

50. King KB, Nail LM, Kreamer K, et al: Patients' descriptions of the experience of receiving radiation therapy. Oncol Nurs Forum 12:55-61, 1985

51. Borthwick D, Knowles G, McNamara S, et al: Assessing fatigue and self-care strategies in patients receiving radiotherapy for non-small cell lung cancer. Eur J Oncol Nurs 7:231-41, 2003

52. Faithfull S: Fatigue in patients receiving radiotherapy. Prof Nurse 13:459-61, 1998

53. Visser MR, Smets EM: Fatigue, depression and quality of life in cancer patients: how are they related? Support Care Cancer 6:101-8, 1998

54. Irvine D, Vincent L, Graydon JE, et al: The prevalence and correlates of fatigue in patients receiving treatment with chemotherapy and radiotherapy. A comparison with the fatigue experienced by healthy individuals. Cancer Nurs 17:367-78, 1994

55. Vogelzang NJ, Breitbart W, Cella D, et al: Patient, caregiver, and oncologist perceptions of cancerrelated fatigue: results of a tripart assessment survey. The Fatigue Coalition. Semin Hematol 34:4-12, 1997

56. Kim YS, Yoon SM, Choi EK, et al: Phase II study of radiotherapy with three-dimensional conformal boost concurrent with paclitaxel and cisplatin for Stage IIIB non-small-cell lung cancer. Int J Radiat Oncol Biol Phys 62:76-81, 2005

57. Farray D, Mirkovic N, Albain KS: Multimodality therapy for stage III non-small-cell lung cancer. J Clin Oncol 23:3257-69, 2005

58. Bush DA, Slater JD, Bonnet R, et al: Proton-beam radiotherapy for early-stage lung cancer. Chest 116:1313-9, 1999

59. Shioyama $\mathrm{Y}$, Tokuuye $\mathrm{K}$, Okumura $\mathrm{T}$, et al: Clinical evaluation of proton radiotherapy for non-small-cell lung cancer. Int J Radiat Oncol Biol Phys 56:7-13, 2003

60. Koto M, Miyamoto T, Yamamoto N, et al: Local control and recurrence of stage I non-small cell lung cancer after carbon ion radiotherapy. Radiother Oncol 71:147-56, 2004

61. Miyamoto $\mathrm{T}$, Yamamoto $\mathrm{N}$, Nishimura $\mathrm{H}$, et al: Carbon ion radiotherapy for stage I non-small cell lung cancer. Radiother Oncol 66:127-40, 2003

62. Nishimura H, Miyamoto T, Yamamoto N, et al: Radiographic pulmonary and pleural changes after carbon ion irradiation. Int J Radiat Oncol Biol Phys 55:861-6, 2003

63. Krengli M, Hug EB, Adams JA, et al: Proton radiation therapy for retinoblastoma: comparison of various intraocular tumor locations and beam arrangements. Int J Radiat Oncol Biol Phys 61:583-93, 2005

64. St Clair WH, Adams JA, Bues M, et al: Advantage of protons compared to conventional X-ray or IMRT in the treatment of a pediatric patient with medulloblastoma. Int J Radiat Oncol Biol Phys 58:727-34, 2004

65. Weber DC, Trofimov AV, Delaney TF, et al: A treatment planning comparison of intensity modulated photon and proton therapy for paraspinal sarcomas. Int J Radiat Oncol Biol Phys 58:1596-606, 2004

66. Baumert BG, Lomax AJ, Miltchev $\mathrm{V}$, et al: A comparison of dose distributions of proton and photon beams in stereotactic conformal radiotherapy of brain lesions. Int J Radiat Oncol Biol Phys 49:1439-49, 2001

67. Zurlo A, Lomax A, Hoess A, et al: The role of proton therapy in the treatment of large irradiation volumes: a comparative planning study of pancreatic and biliary tumors. Int J Radiat Oncol Biol Phys 48:277-88, 2000

68. Miralbell R, Crowell C, Suit HD: Potential improvement of three dimension treatment planning and proton therapy in the outcome of maxillary sinus cancer. Int J Radiat Oncol Biol Phys 22:305-10, 1992

69. Miralbell R, Lomax A, Russo M: Potential role of proton therapy in the treatment of pediatric medulloblastoma/primitive neuro-ectodermal tumors: spinal theca irradiation. Int J Radiat Oncol Biol Phys 38:805-11, 1997 


\section{Chapter 1}

70. Cozzi L, Fogliata A, Lomax A, et al: A treatment planning comparison of 3D conformal therapy, intensity modulated photon therapy and proton therapy for treatment of advanced head and neck tumours. Radiother Oncol 61:287-97, 2001

71. Lee $\mathrm{CH}$, Tait $\mathrm{D}$, Nahum $\mathrm{AE}$, et al: Comparison of proton therapy and conformal X-ray therapy in nonsmall cell lung cancer (NSCLC). Br J Radiol 72:1078-84, 1999

72. Fogliata A, Bolsi A, Cozzi L: Critical appraisal of treatment techniques based on conventional photon beams, intensity modulated photon beams and proton beams for therapy of intact breast. Radiother Oncol 62:137-45, 2002

73. Isacsson $\mathrm{U}$, Montelius A, Jung B, et al: Comparative treatment planning between proton and X-ray therapy in locally advanced rectal cancer. Radiother Oncol 41:263-72, 1996

74. Isacsson $\mathrm{U}$, Hagberg $\mathrm{H}$, Johansson $\mathrm{KA}$, et al: Potential advantages of protons over conventional radiation beams for paraspinal tumours. Radiother Oncol 45:63-70, 1997

75. Lievens Y, Kesteloot K, Van den Bogaert W: CHART in lung cancer: economic evaluation and incentives for implementation. Radiother Oncol 75:171-8, 2005 


\section{PART 1}

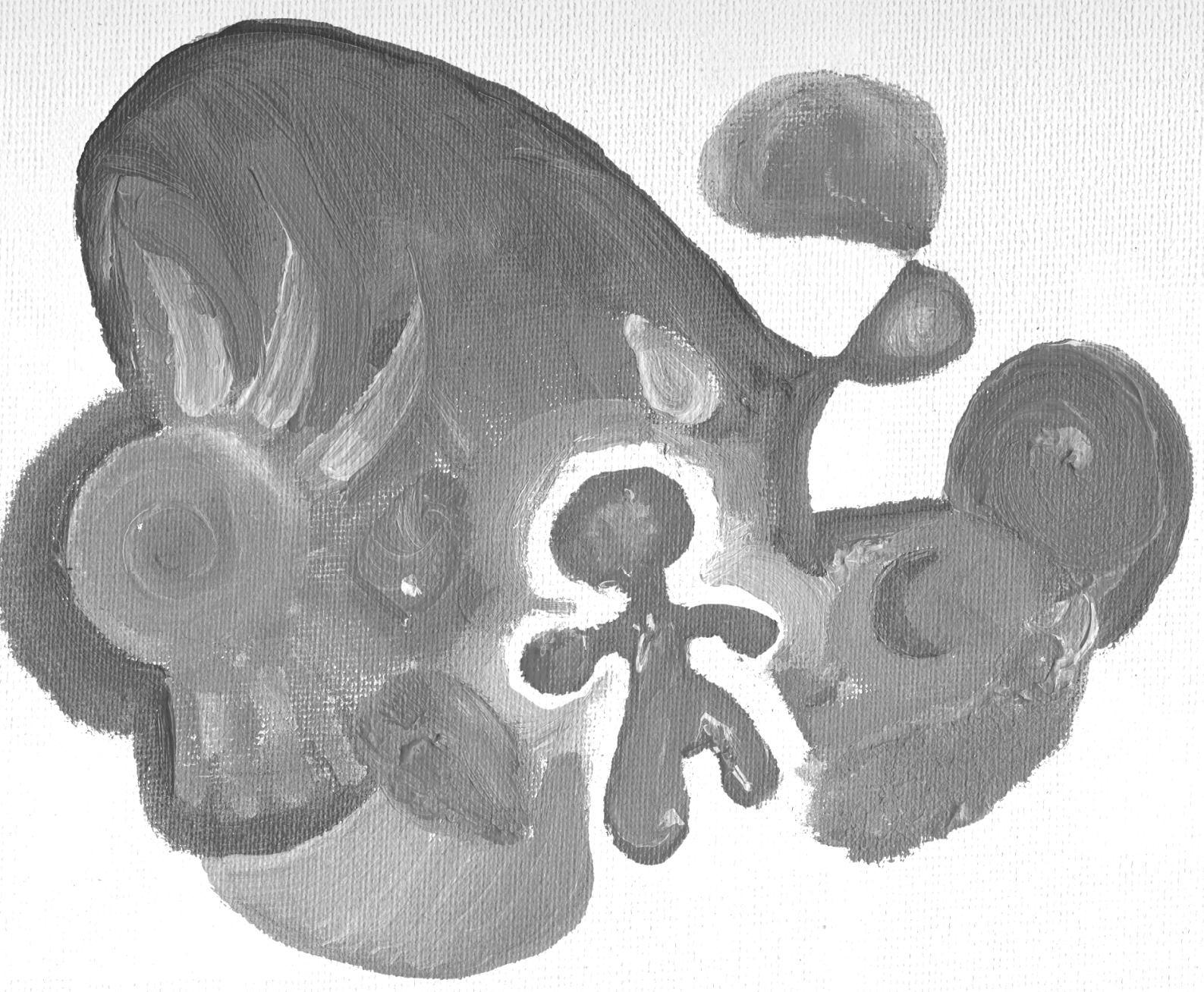




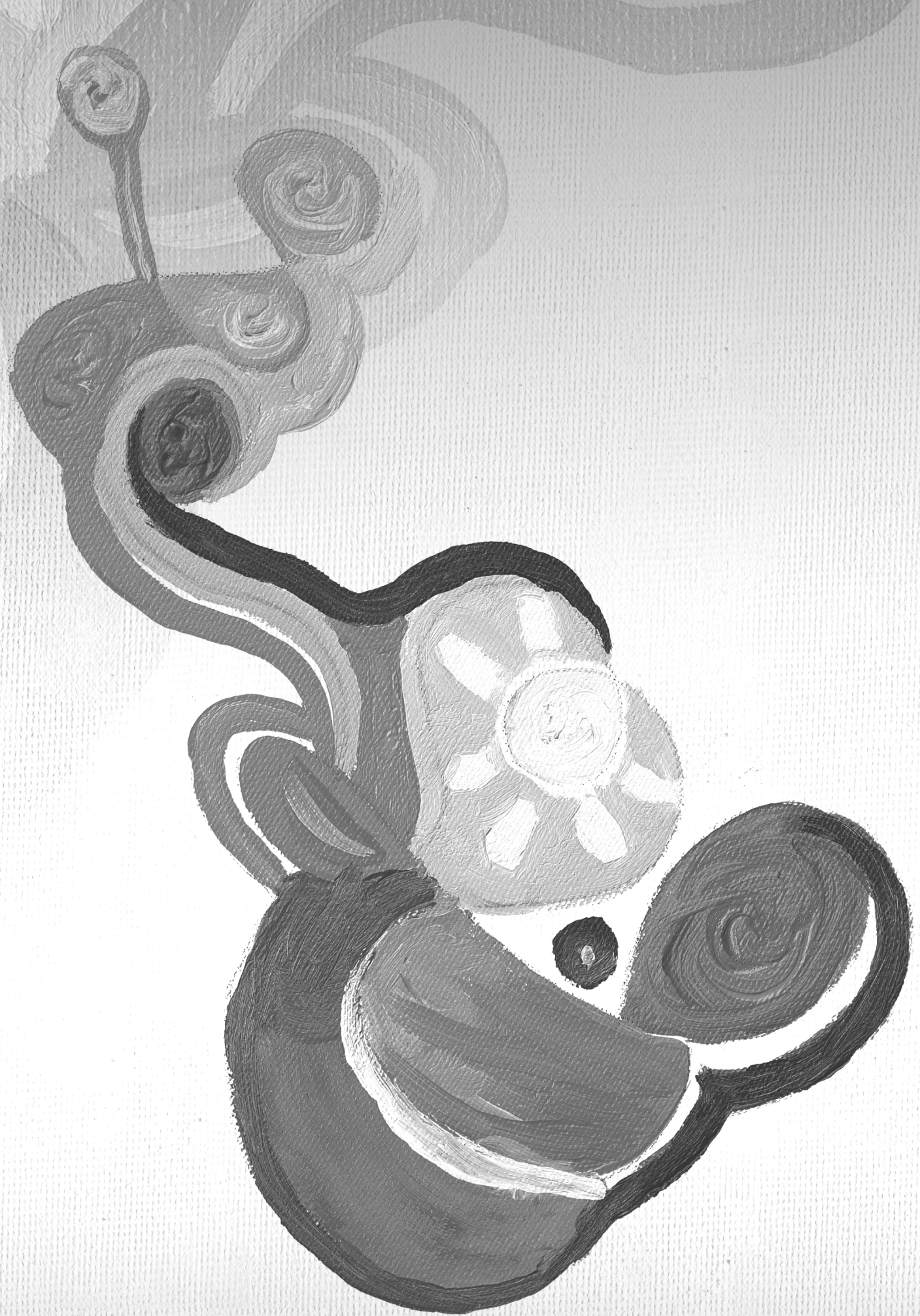




\section{CHAPTER}

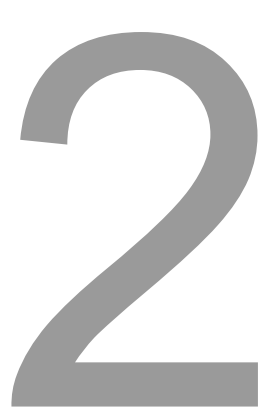

Timing of chest radiotherapy in patients

with limited disease small cell lung

cancer: A systematic review and metaanalysis of randomised controlled trials

Cancer Treatment Reviews (2007), 33: 461- 473

Madelon Pijls-Johannesma

Dirk De Ruysscher

Johan Vansteenkiste

Arnold Kester

Isabelle Rutten

Philippe Lambin 


\section{ABSTRACT}

Background: We undertook a systematic review and literature-based meta-analysis to determine whether the timing of chest radiotherapy may influence the survival of patients with limited disease small cell lung cancer (LD-SCLC).

Objectives: To establish the most effective way of combining chest radiotherapy with chemotherapy for patients with limited-stage small cell lung cancer in order to improve long-term survival.

Materials: Eligible studies were identified according to the Cochrane Collaboration Guidelines and were randomised controlled clinical trials comparing different timing of chest radiotherapy in patients with LD-SCLC. Early chest irradiation was defined as beginning within 30 days afterthe start of chemotherapy.

Results: Seven randomised trials were eligible. The overall survival at 2 years or at 5 years was not significantly different between early or late chest radiotherapy. When only trials were considered that used platinum-based chemotherapy concurrent with chest radiotherapy, significantly higher 2 and 5-year survival rates were observed when chest radiotherapy (RT) was started within 30 days after the start of chemotherapy (2-year survival: HR: $0.73,95 \% \mathrm{Cl} 0.57-0.94, p=0.01$; 5 -year survival: HR: $0.65,95 \% \mathrm{Cl} 0.45-0.93, \mathrm{p}=0.02$ ). This was even more pronounced when the overall treatment time of chest radiotherapy was less than 30 days. In studies that did not show a survival advantage by early chest radiation, a lower dose-intensity of chemotherapy in the early vs. late arm was observed.

Conclusions: When platinum-based chemotherapy concurrently with chest RT is used, the 2-and 5-year survival rates of patients with LD-SCLC may be in favour of early chest radiotherapy, with a significant difference if the overall treatment time of chest radiation is less than 30 days. 


\section{INTRODUCTION}

Small cell lung cancer (SCLC) accounts for about $20 \%$ of all lung cancer cases, with only one third of the patients presenting with limited disease (LD). ${ }^{1,2}$ Without treatment, tumour progression in patients with SCLC is rapid, with a median survival of 2 to 4 months. Chemotherapy has improved the median survival time substantially, but long-term survival remains rare after chemotherapy alone. ${ }^{3}$

Two meta-analyses in LD-SCLC ${ }^{4,5}$ have shown an improvement of $5.4 \%$, in absolute survival at 2-years and 3-years, in patients who received chest irradiation and chemotherapy versus those receiving chemotherapy alone. The 5-year survival rate remains disappointingly low at $10-15 \% .{ }^{4}$ Although evidence for a significant survival benefit of adding chest radiotherapy was provided by the meta-analyses, no conclusions could be drawn regarding the optimal timing and sequencing of chemotherapy and radiation. ${ }^{6,7}$

Using meta-analysis techniques, it appeared that a short time between the first day of chemotherapy and the last day of chest radiotherapy was associated with improved survival in LD-SCLC. ${ }^{8}$ Furthermore, the results of a recently published meta-analysis, suggest that it is essential to ensure that the delivery of chemotherapy is optimal when administered with early chest radiotherapy. ${ }^{9}$

Despite these recent findings, several issues about the administration of chest radiotherapy in LD-SCLC are still unresolved, including its timing with chemotherapy, the optimal overall treatment time of chest radiotherapy, the optimal dose and doseintensity of chemotherapy, and whether or not to deliver radiotherapy concurrent with chemotherapy.

The focus of this systematic review and meta-analysis is the best timing of chest radiotherapy with chemotherapy. Because it has become clear in recent years hat the overall treatment time of radiation plays an important role in the outcome, this variable was also examined. ${ }^{8,10,11}$ Since the type of chemotherapy delivered together with radiotherapy may affect outcomes, we also stratified for this factor. ${ }^{6,12}$

\section{METHODS}

\section{Criteria for selecting studies}

The protocol was peer reviewed and published in the Cochrane Database of Systematic Reviews ${ }^{13}$. Analyses planned in the protocol were: survival, local tumour control, toxicity and compliance. Analyses were stratified according to total treatment time of chest irradiation and by type of combination (sequential or concurrent). Studies eligible for inclusion were randomised controlled clinical trials fully published in journals and those identified from other sources (abstracts and proceedings of rele- 
vant scientific meetings, and contacts with investigators) for which full details are available from investigators. Patients of any age had to have histologically and cytologically proven LD-SCLC and a performance status 0-2. For this review we used the following definition of LD: cancer confined to one hemi-thorax including contralateral mediastinal and hilar lymph nodes as well as ipsilateral and/or bilateral supraclavicular involvement, but excluding malignant pleural effusion.

Early chest radiotherapy was defined as beginning radiation within 30 days after the start of chemotherapy. This choice is made because it has become clear in recent years that accelerated proliferation of tumour clonogens during both radiotherapy and chemotherapy ${ }^{8,10,11,14-16}$, starting from 30 days after the beginning of treatment onwards, affects prognosis.

\section{Search strategy}

A search for identification of studies on the review topic was undertaken using the following electronic databases: the Cochrane Central Register of Controlled Trials (CENTRAL, 2006 Issue 3), MEDLINE (1966 to present), EMBASE (1974 to present), CINAHL (1982 to present). Furthermore, the Cochrane Lung Cancer Groups Specialised Register was consulted. Searches were performed without a language restriction of studies retrieved.

Reference lists from identified studies were scrutinised for any other additional studies. The electronic searches for clinical trials were complemented with manual searches from the following oncology journals: International Journal of Radiation, Oncology, Biology and Physics (1985-present); Radiotherapy and Oncology (1985present); Journal of Clinical Oncology (1985-present); Clinical Oncology (1999present); Lung Cancer (1985-present); and Thorax (1985-present). Abstracts were hand searched from the principal oncology conferences from 1985 onwards with a minimum follow up of 3 years. Colleagues, collaborators, and other experts in the field were asked to identify possibly missing and unreported trials.

\section{Data extraction}

Randomised trials identified by the search were assessed to determine if they met the inclusion criteria. They were assessed by three independent reviewers (DDR, MPJ, JV) both for the quality of the methods against pre-determined criteria (see below) and for the results of key outcomes, which were identified and tabulated.

Two reviewers (DDR, JV) extracted the data independently to ensure validity. Discrepancies were resolved by a third referee (MPJ). All data extracted from randomised trials were included in these analyses. The following data were collected from the manuscript: identifiers, gender, age, performance status at the time of randomisation, initial disease stage, definition of chemotherapy regimen, induction treatment 
that led to a complete response, start date of induction treatment, randomisation timing, treatment allocated, overall treatment time of chest irradiation according to the protocol and updated information on survival, brain metastasis, other metastases and loco-regional recurrence.

\section{Quality assessment}

Methodological quality was assessed according to the following criteria:

Was the randomisation process adequate? Was there adequate allocation concealment? Were the analyses performed according to intention to treat? Were the groups similar at baseline for the most important prognostic indicators? Were eligibility criteria specified? Were losses to follow up fully accounted for and was the withdrawal/drop-out rate unlikely to cause bias? Were co-interventions which may have influenced the results controlled for?

The methodological quality of the included studies is depicted in Table 1.

For local tumour control, there was no uniform definition of local tumour control or of the methods of how it was to be assessed. Analyses were only performed on an intention to treat basis in four of seven studies. ${ }^{17-20}$ The co-interventions that may have influenced the results were not controlled for. We therefore chose the cumulative incidence of local tumour control, which was, if extractable from the paper, derived from the actuarial chest recurrences at 5 years according to randomisation. Only the 5 -year rates were included because these will probably not be influenced by second line chemotherapy given for recurrence after primary treatment.

\section{Statistical Analysis}

We performed a literature-based meta-analysis. A weighted estimate of the typical treatment effect across studies was computed for 2-year survival data as well as the 5 -year survival data, the cumulative local tumour control at 5 years and toxicities (incidence of haematological, lung- and esophageal grade 3-4 toxicity, if available or extractable from the manuscript).

For survival, the hazard ratio (HR) was used as the effect measure, whereas for all other outcomes the odds ratio (OR) was used. Chi-square heterogeneity tests were used to test for statistical heterogeneity among trials. Analysis was performed using the fixed effect model, but in case of heterogeneity, a random effect model was used. For the study of Spiro ${ }^{9}$, only 3-year survival data were available. For the clarity of the tables and text, these were set under the denominator "2-year survival". 


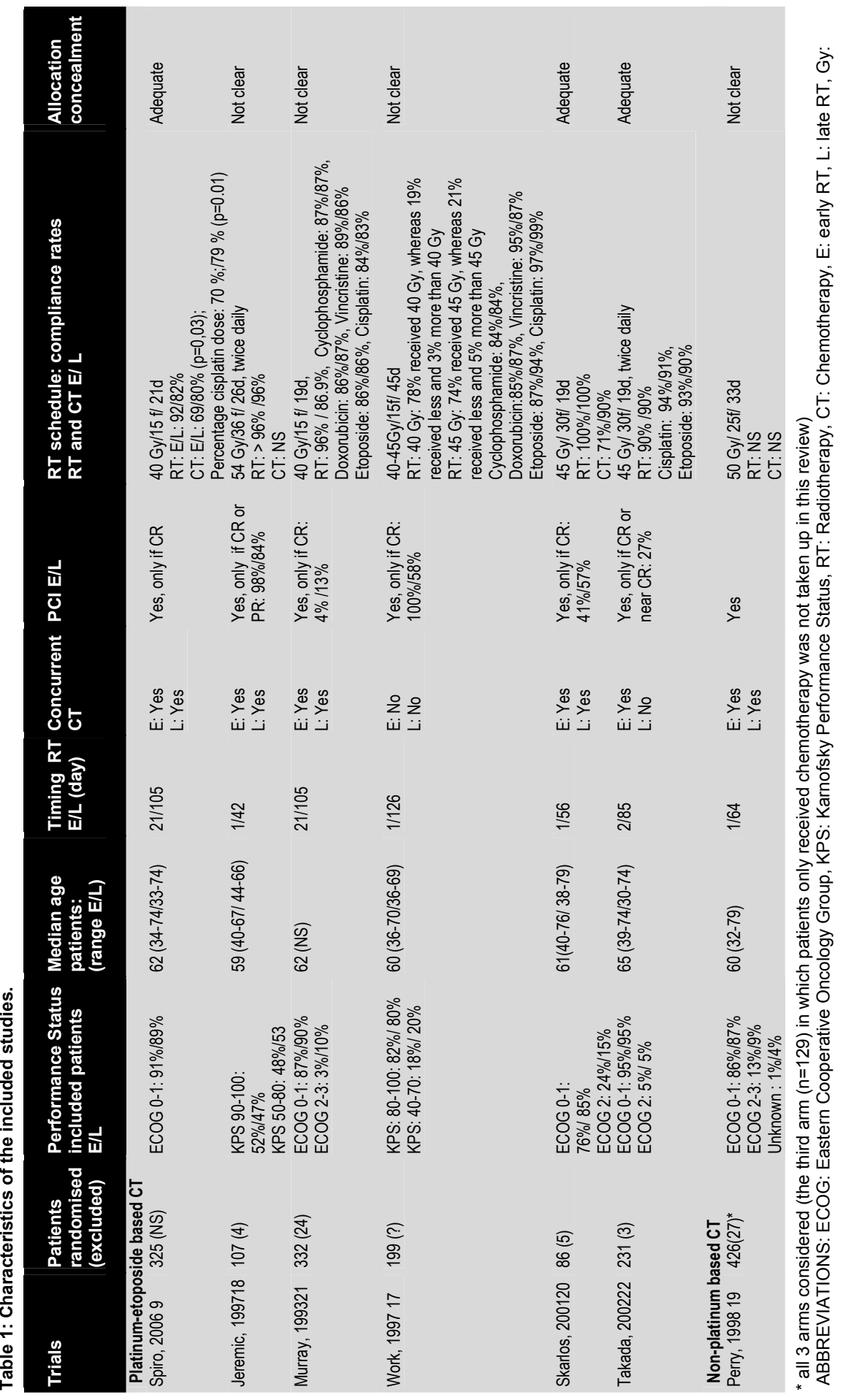


Post-hoc analysis were carried out according to whether platinum-based chemotherapy was used during chest radiotherapy or not. We also investigated whether or not chemotherapy was delivered with a reasonable total dose and dose-intensity. 'Reasonable' total dose and dose intensity of chemotherapy was defined as a total dose and dose intensity of $85 \%$ or more.

Sources of heterogeneity in the assessment of the primary outcome measure were explored by random effects meta-regression. These analyses assessed the effect of the overall treatment time of chest irradiation and of the concurrent administration of chemotherapy. This procedure gives an indication of the robustness of the results.

All statistical analysis were performed with RevMan (Review Manager (RevMan) software Version 4.2 for Windows. Oxford, England: The Cochrane Collaboration, 2003) and STATA version 8.0 for Windows.

\section{RESULTS}

The search strategy identified eleven studies. From these 11 studies, 7 were suitable for survival analysis ${ }^{9,17-22}$ and four trials were excluded. ${ }^{23-26}$ Two were excluded because chest radiotherapy started on the same day, thus making comparison between early and late radiation impossible. ${ }^{24,26}$ The study of the EORTC ${ }^{23}$ was not suitable for this analysis because in one arm chest radiotherapy started on day 49 and in the other arm on day 91, implying that according to our inclusion criteria in both arms late radiotherapy was delivered. Likewise, the trial of Lebeau ${ }^{25}$ was excluded because the start of chest radiotherapy varied between day 30-64 in one arm and day 36-47 or day 64-75 in the other arm.

From the seven studies suitable for survival analysis, five gave enough information for local toxicity analysis, i.e. severe pneumonitis and severe oesophagitis ${ }^{9,18-21}$ and six studies for haematological toxicity evaluation. ${ }^{9,17-20,22}$ Local tumour control data were available from 5 of these 7 studies $^{17-19,21,22}$. However, in the study from Japan 22 , only the site of first recurrence was available, whereas in the other trials, the cumulative rate of local tumour failure at 2 and 5 years was available. Compliance, defined as the percentage of intended total radiation dose completed, was registered in 5 of these 7 studies. ${ }^{17,18,20-22}$ In only one of the six studies, chemotherapy was not delivered concomitantly with chest radiotherapy. ${ }^{17}$ In another trial ${ }^{22}$, the early chest radiotherapy group received concurrent radiation and chemotherapy, whereas the late radiation group only had their radiotherapy after chemotherapy. As a consequence, there are not enough trials available to investigate the effect of the sequencing of chemotherapy and thoracic radiotherapy on survival. As in all studies, prophylactic cranial irradiation ( $\mathrm{PCl}$ ) was given to at least a group of patients, it was not possible to stratify for this parameter. 


\section{Compliance}

\section{Compliance with chest radiotherapy}

The percentage of intended total radiation dose completed was registered in six studies. $9,17,18,20-22$ In the study of Spiro et al, RT according to the protocol was completed significantly more frequently in the early group $147 / 159$ (92\%) compared to the late group136/166 (82 \%) $(p=0.01)$. The lowest compliance $(74 \%$ to $78 \%)$ was observed in the Danish Trial ${ }^{17}$, whereas the highest $(100 \%)$ was observed in the study of Skarlos et $\mathrm{al}^{20}$ The results of compliance, both for radiotherapy and chemotherapy, are shown in table 1.

\section{Compliance with chemotherapy}

Overall, the compliance rates were high and similar between early and late thoracic irradiation (Table 2). However, in the studies of Skarlos et al ${ }^{20}$ and Spiro et $\mathrm{al}^{9}$, the compliance rate in the early group was considerably lower compared to that of the late radiation group in two studies $(71 \%$ vs $90 \%$ and $69 \%$ vs $80 \%(p=0.03)$ respectively). In this latter study, the percentage of the delivered cisplatin dose was significantly lower in the early compared to the late radiotherapy group, $70 \%$ versus $79 \%$ respectively $(p=0.01)$. A post-hoc analysis showed that the 2 -year survival was not statistically different between these low and high compliance groups (HR:1.12, 95\% Cl 0.69-1.82).

\section{Dose-intensity chemotherapy}

The dose and intensity of the chemotherapy of the included studies is depicted in Fig.5.

In three studies the required delivered dose of $85 \%$ was not reached. In the study of Spiro et al, cisplatin and etoposide , were delivered at a dose-intensity of $74 \% / 81 \%$ (early /late, E/L) and $70 \% / 79 \%$ (E/L) respectively ${ }^{9}$. Cisplatin was below $85 \%$ in trial of Murray et al ( $E / L 84 \% / 83 \%)^{21}$, and this was the same for cyclophosphamide in the study of Work et al ( $\mathrm{E} / \mathrm{L} 84 \% / 84 \%) .{ }^{17}$ Because the variance of the delivered doses between both arms was low, it was not possible to stratify for this factor. 


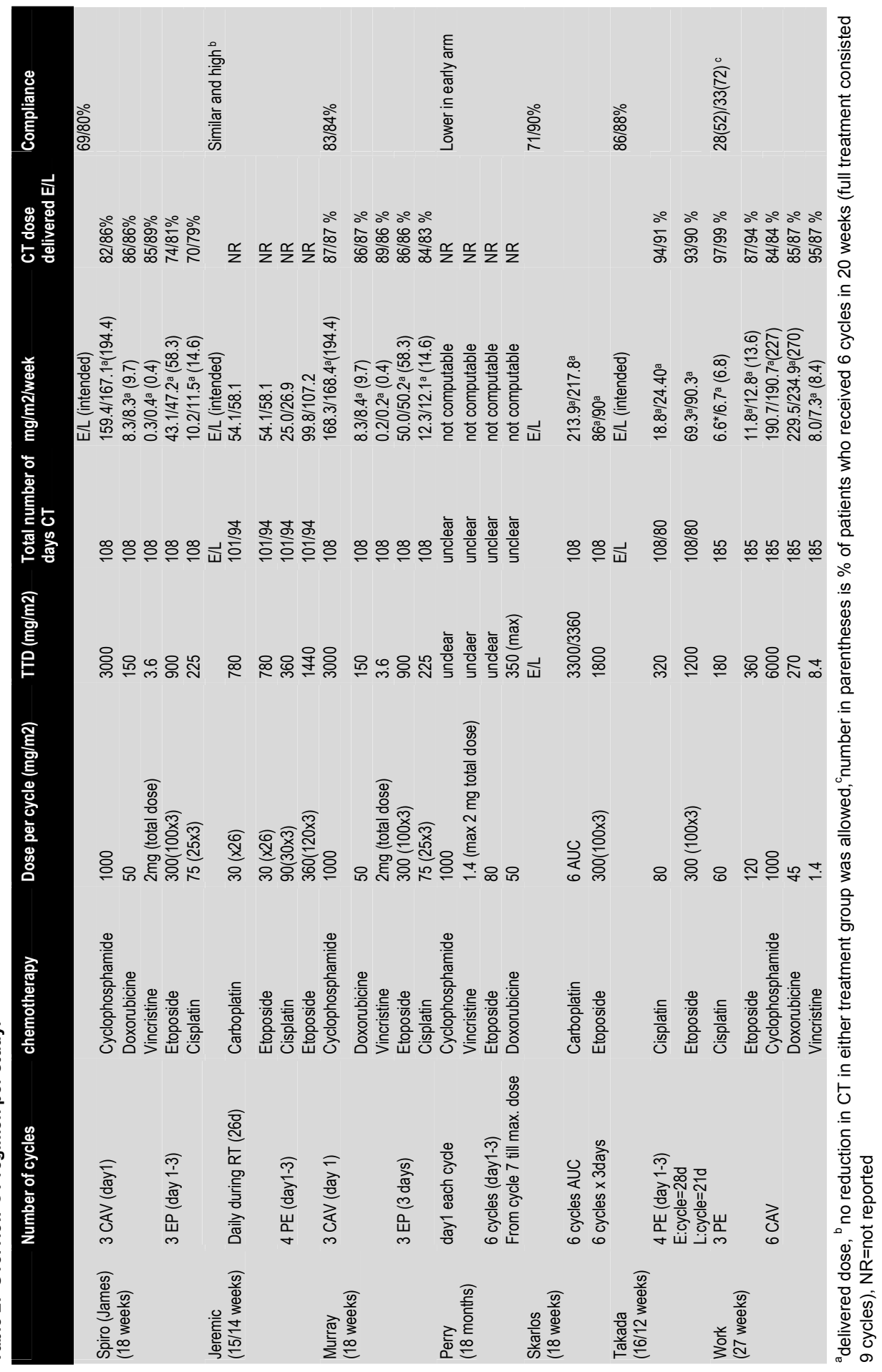




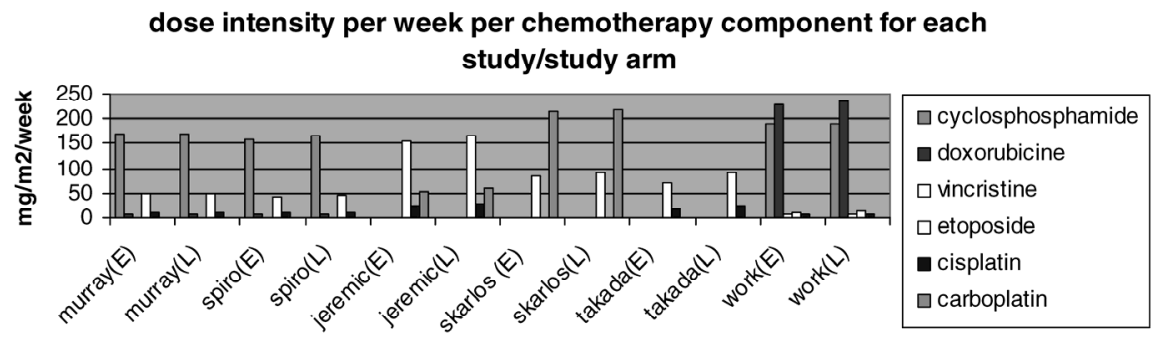

Figure 5 Distribution chemotherapy dose per study arm

\section{Survival}

Taking all seven studies into account, the overall survival at 2 years or at 5 years was not significantly different between early or late chest radiotherapy (Figure 1a). When the one trial that delivered non-platinum chemotherapy concurrently with chest radiation ${ }^{19}$ was excluded, the hazard ratio (HR) was significantly different at 2 years (HR: $0.73,95 \% \mathrm{Cl} 0.57-0,94, \mathrm{p}=0.01$ ), and was also significantly in favour of early chest radiotherapy at 5 years (HR: $0.65,95 \% \mathrm{Cl} 0.45-0.93, \mathrm{p}=0.02)$ (Figure $1 \mathrm{~b})$. Considering studies with an overall treatment time of chest radiation of less than 30 days 9,18,20-22, the 5-year survival (HR: $0.57,95 \% \mathrm{Cl}: 0.38-0.85$; $\mathrm{p}=0.005$ ), but not the 2 year survival, was significantly higher for early chest radiotherapy (Figures $2 a$ and $2 b)$.

\section{Local tumour control}

All included studies determined the local tumour control by chest X-rays or CT-scans. No significant effect of delivering chest radiotherapy early or late on local tumour control was observed (Figure 3). The same held true when the study combining nonplatinum based chemotherapy with thoracic radiotherapy ${ }^{19}$ was omitted.

\section{Toxicity}

Only a trend for a higher incidence for severe pneumonitis was observed for early chest radiotherapy compared with late irradiation when all four studies ${ }^{18-21}$ were considered (OR: 2.02: 95\% Cl: 1.01-5.05; p=0.05) (Figure 4a). A higher incidence of severe esophagitis was observed for early chest radiotherapy when all five studies 9,18-21 were taken into account (OR: 1.49: 95\% Cl: 1.02-2.16; $p=0.04$ ) (Figure 4a). 


\section{A}

$\begin{array}{ll}\text { Review: } & \text { Early versus Late Chest Radiotherapy in patients with limited stage small cell lung cancer (Version CTR febr.07) } \\ \text { Comparison: } & 01 \text { Early versus Late Chest RT }\end{array}$

Outcome: $\quad 15$ 2-3-yrs Survival (death within 2 or 3 years)

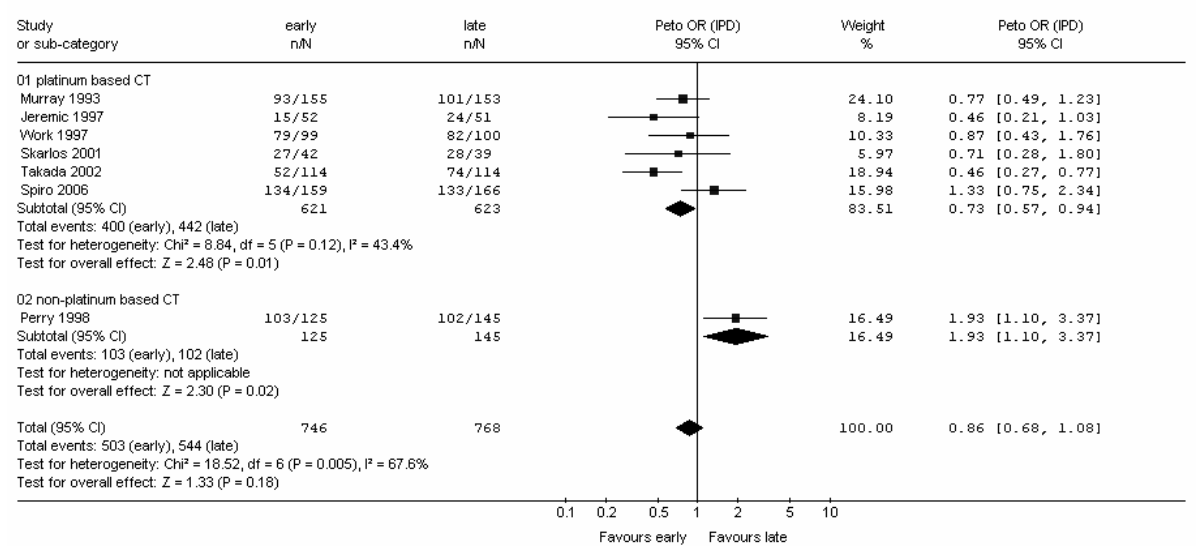

\section{B}

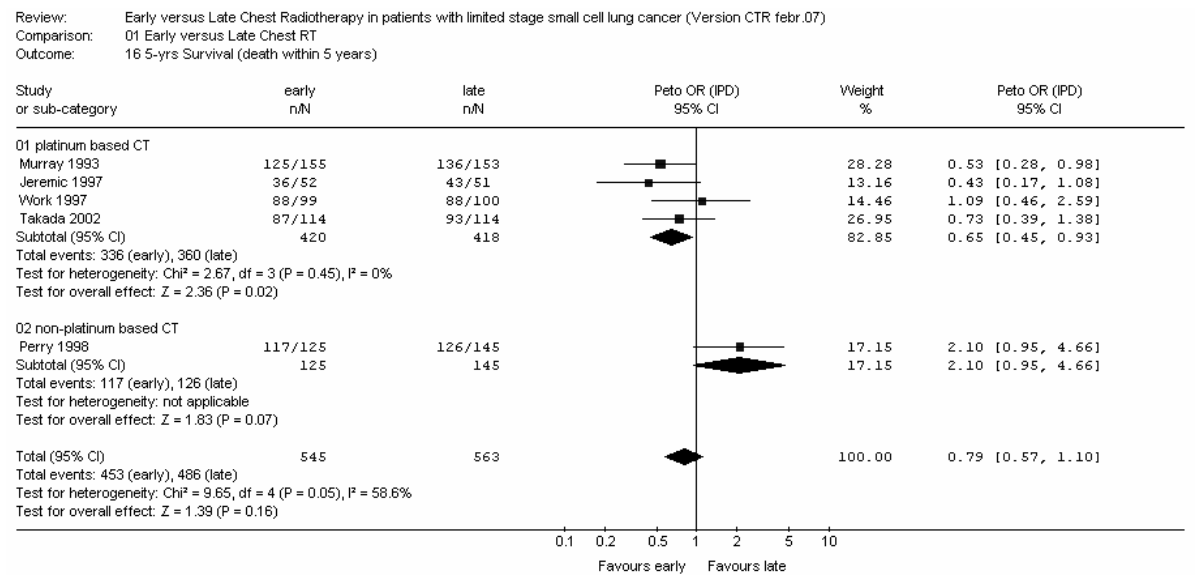

Figure 1 Meta-analysis of survival as a function of the timing of chest radiotherapy. (A) 2-3 year survival, (B) 5year survival

The same was observed when the one trial that delivered non-platinum chemotherapy concurrently with chest radiation ${ }^{19}$ was excluded (Figure $4 b$ ), or when taking into account studies with an overall treatment time of chest radiation of less than 30 days (Figure 4c) ${ }^{9,18,20,21}$. Taking all studies into account ${ }^{9,17-22}$, severe leukopenia was significantly more frequent in patients receiving early chest radiotherapy (OR: 2.56: 95\% Cl: 2.00-3.27; $p<0.00001$ ), whereas severe thrombocytopenia was not (Figure 4a). The same observation was found when only platinum chemotherapy delivered concurrently with radiotherapy was investigated (Figure $4 \mathrm{~b}$ ), or only studies with an over- 
all treatment time of chest radiation of less than 30 days were taken into account (Figure 4c).

\section{Meta-regression}

Sources of heterogeneity in the assessment of the primary outcome measure were explored by random effects meta-regression. The effect of the overall treatment time of chest irradiation and the concurrent or non-concurrent administration of chemotherapy were assessed. Since there was only one study that delivered non-platinum based chemotherapy concurrently with chest radiation ${ }^{19}$, no meta regression analyses could be performed to determine the association between delivering nonplatinum based or platinum based chemotherapy concurrently with chest radiation on overall survival, local control, local toxicities and haematological toxicities.

However, we identified the concurrent delivery of non-platinum based chemotherapy with chest radiation, as a potential source of the observed heterogeneity. Regarding the effect of the overall treatment time on survival, five studies delivered the radiation with an overall treatment time less than 30 days ${ }^{9,18,20-22}$ and in two studies the overall treatment time was more than 30 days. ${ }^{17,19}$ We found a significant association between overall treatment time and overall survival in favour of an overall treatment time less than 30 days, though only on the 5-years survival, not on 2-years survival (5-year survival: regression coefficient -1.02 ; SE $0.38 ; p=0.007 ; 95 \% \mathrm{Cl}:-1.75$, 0.28 ). This finding is in line with our findings in an earlier meta-analysis that a short time between the first day of chemotherapy and the last day of chest radiotherapy was associated with improved survival in LD-SCLC. ${ }^{8}$ We found no association between overall treatment time of chest radiotherapy on local tumour control, local toxicity and haematological toxicity.

\section{DISCUSSION}

From two meta-analyses, it has become clear that adding chest radiotherapy to chemotherapy in patients suffering from limited disease small cell lung cancer (SCLC) improves survival. ${ }^{4,5}$

However, several issues about the administration of thoracic radiotherapy in LDSCLC are still unresolved, including timing with chemotherapy., 7

We therefore focussed this review on timing of chest radiotherapy in conjunction with chemotherapy. In recent years it has become clear that the overall treatment time of radiation plays an important role in the outcome of radiotherapy ${ }^{10,11}$, in addition to the timing of chest radiotherapy. ${ }^{8}$ Therefore, we decided to examine the overall treatment time of chest irradiation as well. 
A

Review: Early versus Late Chest Radiotherapy in patients with limited stage small cell lung cancer (Version CTR febr. 07)

Comparison: $\quad 01$ Early versus Late Chest $R T$

Outcome: 17 2-3-yrs Survival (death within 2 or 3 years) only studies with OTT of chest RT less than 30 days

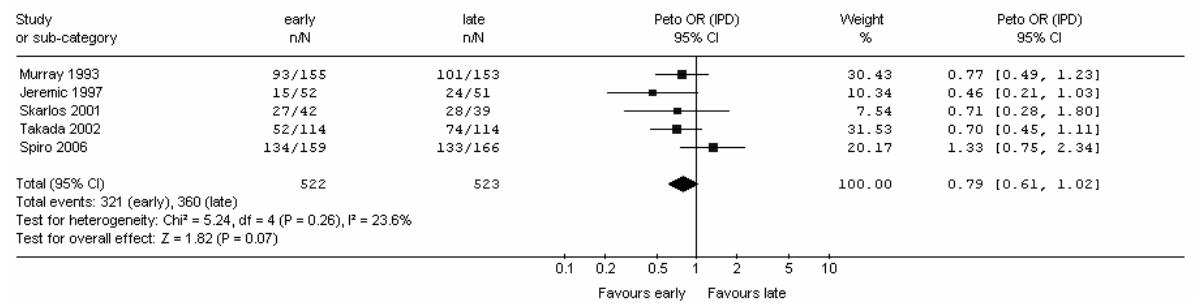

B

Review: Early versus Late Chest Radiotherapy in patients with limited stage small cell lung cancer (Version CTR febr. 07)

Comparison: 01 Early versus Late Chest RT

Outcome: 185 -yrs Survival (death within 5 years) only studies with OTT of chest RT less than 30 days

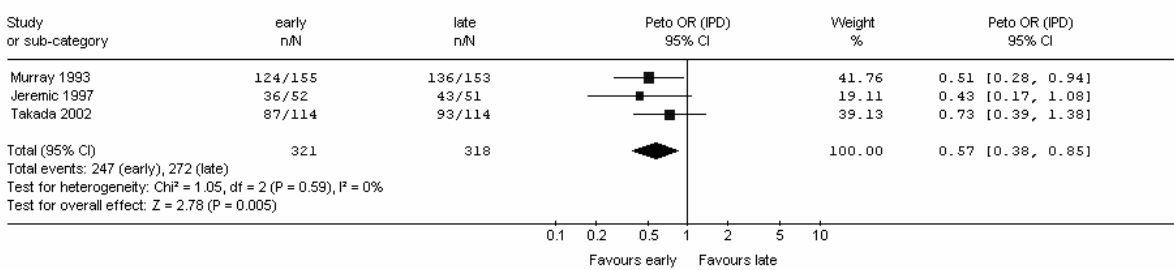

Figure 2 Meta-analysis of survival as a function of the timing of chest radiotherapy. Overall treatment time of thoracic radiation less than 30 days. (A) 2-3 year survival, (B) 5-year survival

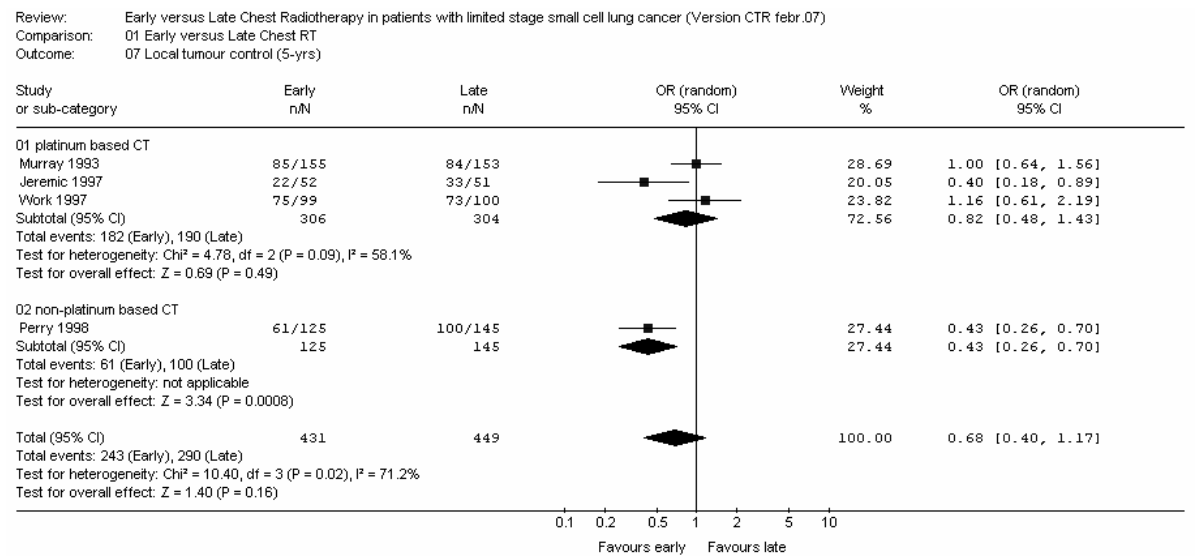

Figure 3 Meta-analysis of local tumor recurrence as a function of the timing of chest radiotherapy.

Only scarce data are available on tumour cell clonogen proliferation of SCLC during and after chemotherapy and radiotherapy. ${ }^{8}$ The results suggest that as in many common solid tumours, accelerated tumour cell clonogen proliferation occurs approximately 30 days after the start of effective cytotoxic therapy. ${ }^{8,10,11,14}$ We therefore defined early chest radiotherapy as starting chest irradiation before 30 days after the 
A Review: $\quad$ Early versus Late Chest Radiotherapy in patients with linited stage small cell lung cancer (Version CTR febr. 07)
Comparison: 01 Early versus Late Chest RT Outcome: $\quad 03$ toxicity, all studies

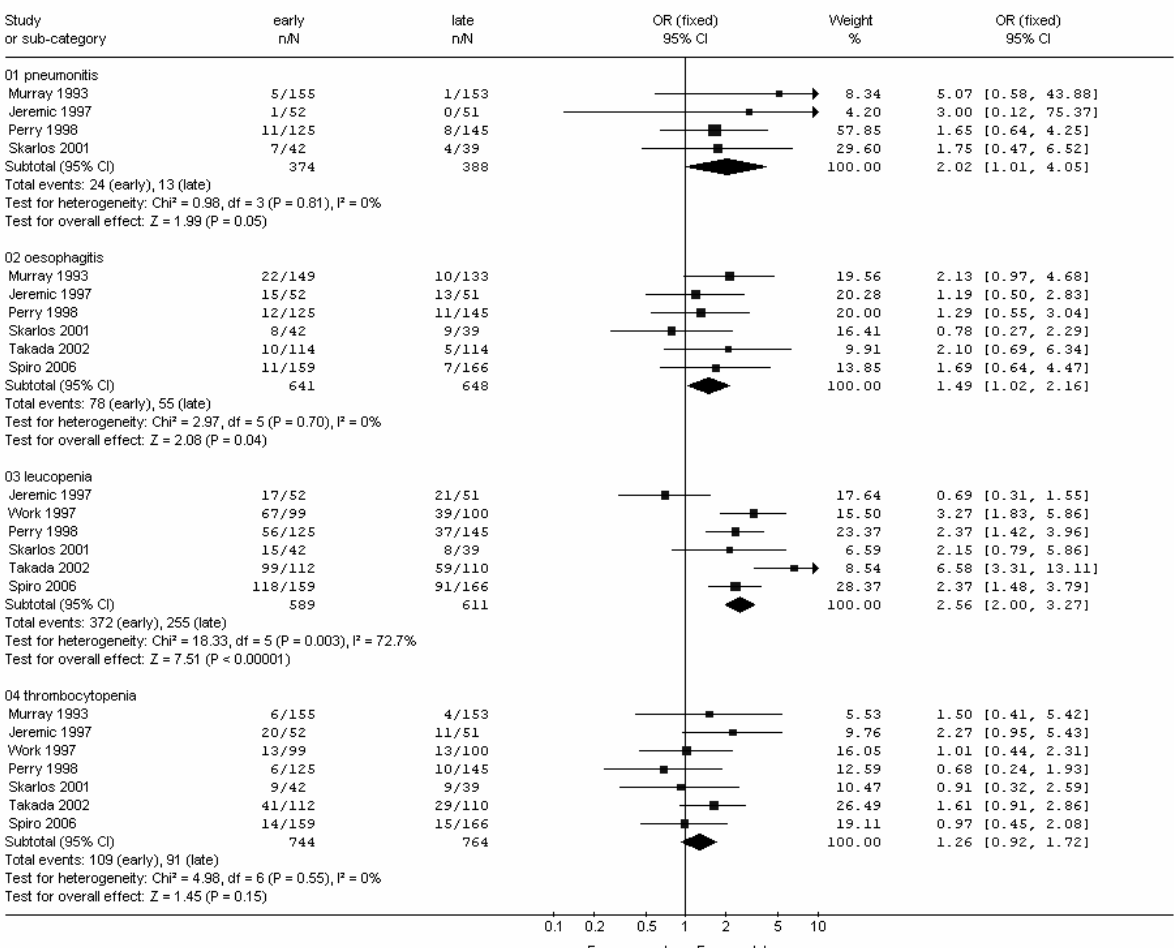

Figure 4 Meta-analysis of severe pneumonitis, esophagitis, leukopenia and thrombocytopenia as a function of the timing of chest radiotherapy. (A) All studies, (B) Only Platinum concurrent with chest radiotherapy, and (C) Only chest radiotherapy with an overall treatment time of less than 30 days.

start of chemotherapy. Late radiotherapy was defined as starting chest irradiation more than 30 days after the start of chemotherapy. Since the type of chemotherapy delivered together with radiotherapy may affect outcomes, we also stratified for this. ${ }^{6,12}$

Seven studies enabling investigating the best timing of chest radiotherapy for survival were identified. ${ }^{9,17-22}$ Four well-known trials were excluded for this analysis. In two of them, chest radiotherapy started on the same day in both arms of the trial, thus making comparison between early and late radiation impossible. ${ }^{24,27}$ In another two studies, thoracic radiotherapy started in both arms after day 30 , implying that, according to our inclusion criteria, in both arms late radiotherapy was delivered. ${ }^{23,25}$

From the seven studies suitable for survival analysis, four gave enough information for severe pneumonitis analysis ${ }^{18-21}$; five for severe esophagitis analysis ${ }^{9,18-21}$; and six studies for haematological toxicity evaluation. .,17-20,22 $^{2}$ 


\section{B}

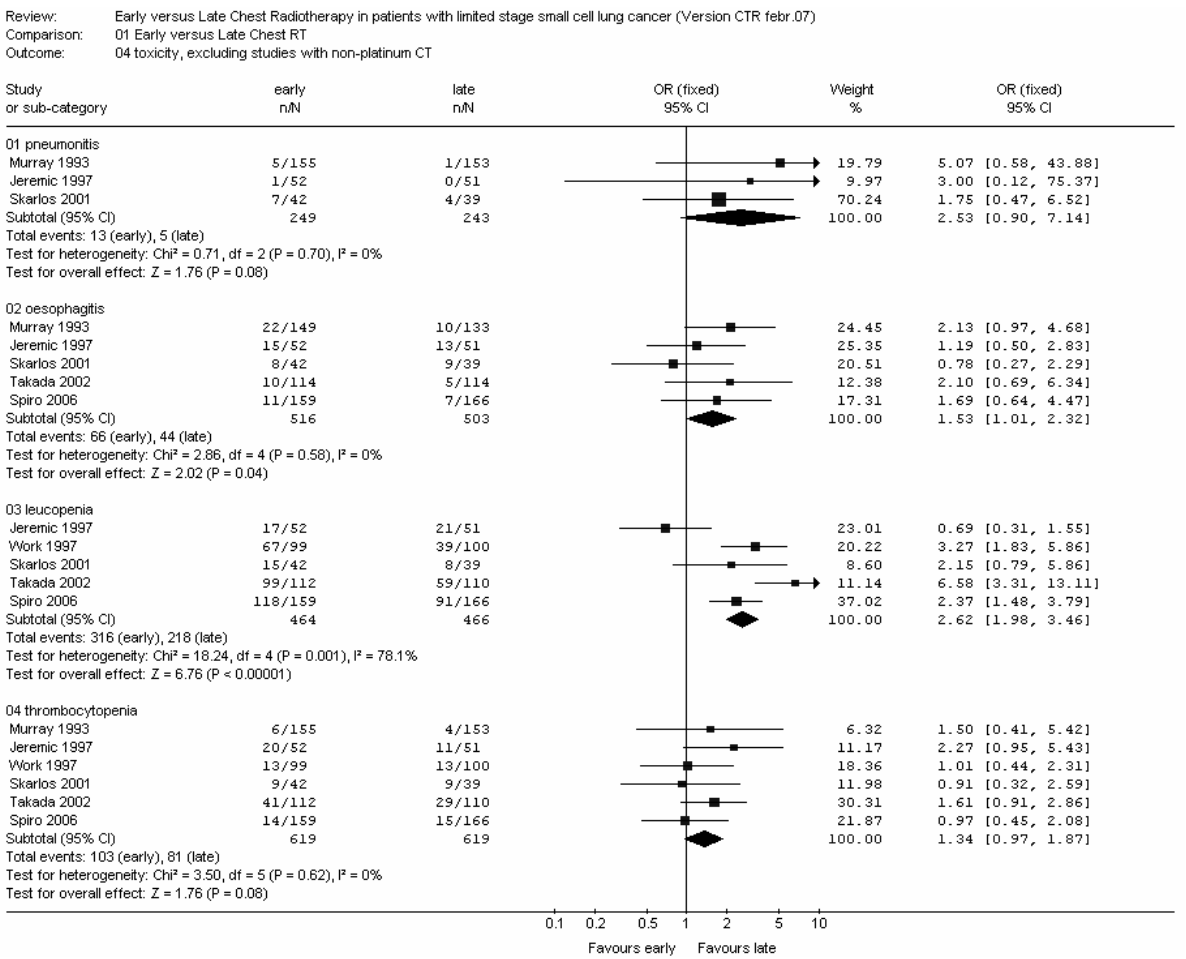

Figure 4 (continued)

Local tumour control data were available from five of these seven studies. ${ }^{17-19,21,22}$ However, in the study from Japan ${ }^{22}$, only the site of first recurrence was available, whereas in the other trials, the cumulative rate of local tumour failure at both two and five years was available.

The five-year data were considered to be the most suitable for analysis, for they are the least influenced by other confounding factors such as chemotherapy given for recurrence.

In one of the seven studies, chest radiotherapy was only partly delivered during chemotherapy cycles in one arm, while in the second arm it was given after the end of chemotherapy. $^{17}$

In another trial ${ }^{22}$, the early chest radiotherapy group received concurrent radiation and chemotherapy, while the late radiation group only received radiotherapy after chemotherapy. As a consequence, there are not enough trials available to investigate the effect of the sequencing of chemotherapy and thoracic radiotherapy on survival. As in all studies $\mathrm{PCl}$ was given to at least one group of patients, it was not possible to stratify for this variable either. 
C

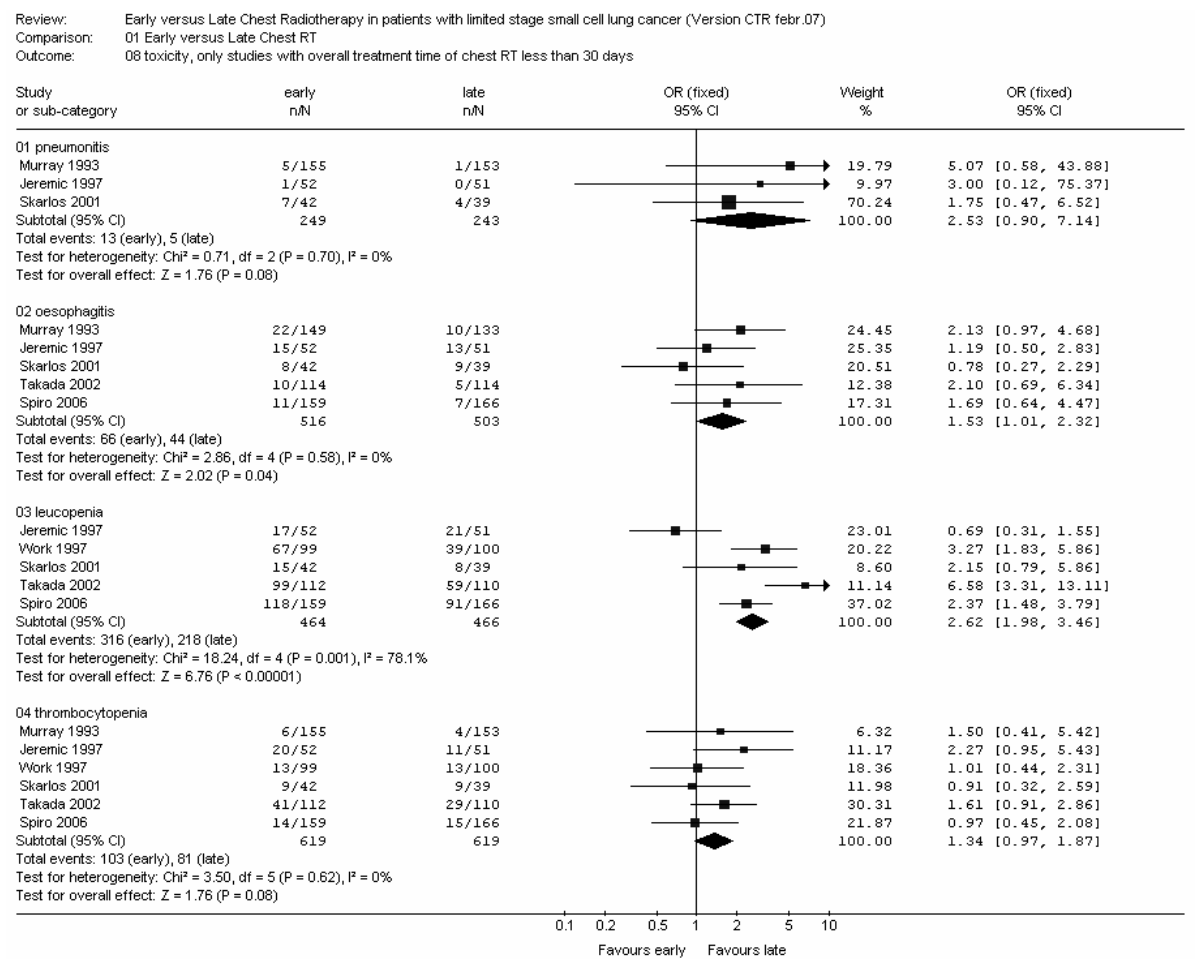

Figure 4 (continued)

The effect of the timing of chest radiotherapy on survival is not clear. For all studies together, no significant differences in either the two-year or the five-year survival rates were observed. When the only trial in which non-platinum chemotherapy was delivered concurrently with chest radiotherapy was excluded ${ }^{19}$, survival was significantly in favour of beginning thoracic radiation within 30 days after the start of chemotherapy. This finding would represent a five-year survival rate of $20.2 \%$ for early versus $13.8 \%$ for late chest radiotherapy. However, these results should be interpreted with caution. First, the subgroups based on non-platinum chemotherapy delivered during radiotherapy versus platinum-based chemotherapy were a post-hoc analysis by our group, and secondly, based on a comparison with only one trial that did not use platinum-based CT. Thirdly, the five-year data rely to a large extent on the $\mathrm{NCl}-\mathrm{C}$ trial ${ }^{21}$. A subsequent large study ${ }^{9}$ with the same design and therapeutic regimen as the $\mathrm{NCl}-\mathrm{C}$ trial failed to show a survival difference at three years between early and late chest radiotherapy. In this trial, however, patients randomised to early 
chest radiation received significantly less chemotherapy than in the late arm. Furthermore, the five-year survival rates of this study are still awaited. Fourthly, the studies with the highest reported dose intensity of chemotherapy seem to have the highest survival rates and show the largest differences between early and late chest radiotherapy. ${ }^{18,20-22}$ The same studies also report the highest compliance rates. It seems that in order to have a benefit of early chest radiotherapy, an effective systemic treatment as well as a two-year survival of at least $30 \%$ should be achieved. It is difficult to explain these findings, which may be interrelated. It is not unlikely that the beneficial effect of early chest radiation can only be observed in those patients in a good general condition, thoroughly staged, and thus well compliant for an intensive, but effective chemotherapy regimen combined with chest radiotherapy. However, this hypothesis cannot be proved by the available data. At present, it is unclear whether early chest radiotherapy confers a survival benefit. In the five studies with an overall chest radiation treatment time of fewer than 30 days ${ }^{9,18,20-22}$, the five-year survival rates significantly indicated the benefits of early chest radiotherapy, but not the twoyear rates. As the results at five years are to a large extent driven by the study of Murray et al (1993), whose findings have not been confirmed by the study of Spiro et al (2006) at three years, the results should be interpreted with caution. No firm conclusion can be drawn as to whether the overall treatment time of chest radiotherapy affects survival or not.

The different result in outcome between the study of Spiro et al and Murray et al, which used the same study design and treatment schedules, cannot not be explained by differences in the delivered dose intensity per treatment arm, since these were similar per study. However, the delivered dose of etoposide-cisplatin was considerable lower in the study of Spiro et al (Table 2). The most obvious factor to explain the different results between these two studies is thus the compliance to chemotherapy. To determine the effect of the dose-intensity of chemotherapy on treatment outcome in the combined modality treatment with radiotherapy, further research is required.

Local tumour control was not significantly different between early and late chest radiotherapy. This may seem surprising in considering the five-year results, as a significant survival benefit was observed in favour of early chest radiotherapy. This discrepancy may be due to the fact that local control figures were much influenced by the data of the study of Murray et al (1993), who observed a significantly better rate of survival for patients receiving early chest radiotherapy versus late radiation, but no significant difference in local tumour control. In the Danish trial ${ }^{17}$, no differences between early and late radiotherapy were observed, but chest radiotherapy was not delivered concomitantly with chemotherapy, which might have influenced the results. Moreover, two of the four studies amenable for local tumour control evaluation had an overall treatment time of chest radiation of more than 30 days ${ }^{17,19}$ which also may 
have increased the heterogeneity among the studies. At present, no 5 year data on the local tumour control rate are available from the largest trial. ${ }^{9}$

Keeping all caveats for comparing local toxicity between studies in mind, severe pneumonitis and severe esophagitis were not significantly different between early and late chest radiotherapy, whether non-platinum chemotherapy was administered during chest radiotherapy or not ${ }^{9,18-22}$, but a trend for a higher incidence of pneumonitis when delivering early chest radiotherapy was observed. It may be that larger radiation fields were used when irradiation was carried out during the first or the beginning of the second chemotherapy cycles, at a time when the primary tumour and the lymph node metastases were still larger than after later chemotherapy cycles. Even when the overall treatment time of chest radiation was less than 30 days $9,18,20,21$, severe local toxicity was not significantly different between early and late chest radiotherapy.

Compliance rates of the studies, except for the early arm of the studies of Skarlos et al and Spiro et al, were high $(87 \% \text { to } 100 \%)^{18,21,22}$, which implies that these radiotherapy schedules are well tolerated.

Overall, the delivered dose intensity per week of the chemotherapy appeared similar in both treatment arms per study, but considerable differences between dose schedules and regimens of the included studies were observed (Figure 5).

Severe leukopenia was significantly more frequent in patients receiving early chest radiotherapy, regardless of whether all studies were taken into account, or the study delivering concurrent non-platinum chemotherapy during chest radiation ${ }^{19}$ was excluded, or when the overall chest radiotherapy treatment time was fewer than 30 days. This finding, however, may be attributed to the increased bone marrow damage when delivering concurrent chemotherapy and chest radiotherapy, which both cause leukopenia. Severe thrombocytopenia was not significantly different between early and late chest irradiation when all studies were considered. When the one trial that delivered non-platinum chemotherapy concurrently with chest radiation ${ }^{19}$ was excluded, or when taking into account only studies with an overall treatment time of chest radiation of fewer than 30 days ${ }^{9,17,18,20,22}$, a trend for more severe thrombocytopenia for early chest radiotherapy appeared. Once again, this may be due to bone marrow suppression caused by earlier chemotherapy treatments.

In conclusion, the possible benefit of early chest radiotherapy (beginning within 30 days after the start of chemotherapy) on 5-year survival should be viewed with caution. Early chest radiotherapy could be more effective - but as the compliance rate appeared to be very important - the influence of patient selection and of the systemic treatment should be taken into account. Early chest radiotherapy leads to a higher incidence of severe leukopenia, while late chest radiotherapy showed a trend for more thrombocytopenia in some subgroups. 
In view of the many uncertainties, more research is needed to investigate the optimal timing of chest radiotherapy, the best radiotherapy schedule in relation to the type of chemotherapy chosen, the optimal delivering of chemotherapy, the volumes to be treated and the patient groups for whom these treatments apply. 


\section{REFERENCES}

1. Bunn PA, Jr., Carney DN. Overview of chemotherapy for small cell lung cancer. Semin Oncol 1997;24(2 Suppl 7):S7-69-S7-74.

2. Stupp R, Monnerat C, Turrisi AT, 3rd, Perry MC, Leyvraz S. Small cell lung cancer: state of the art and future perspectives. Lung Cancer 2004;45(1):105-17.

3. Kelly K. New chemotherapy agents for small cell lung cancer. Chest 2000;117(4 Suppl 1):156S-162S.

4. Pignon JP, Arriagada R, Ihde DC, et al. A meta-analysis of thoracic radiotherapy for small-cell lung cancer. N Engl J Med 1992;327(23):1618-24.

5. Warde $P$, Payne D. Does thoracic irradiation improve survival and local control in limited-stage smallcell carcinoma of the lung? A meta-analysis. J Clin Oncol 1992;10(6):890-5.

6. Kumar $P$. The role of thoracic radiotherapy in the management of limited-stage small cell lung cancer: past, present, and future. Chest 1997;112(4 Suppl):259S-265S.

7. De Ruysscher D, Vansteenkiste J. Chest radiotherapy in limited-stage small cell lung cancer: facts, questions, prospects. Radiother Oncol 2000;55(1):1-9.

8. De Ruysscher D, Pijls-Johannesma M, Bentzen SM, et al. Time between the first day of chemotherapy and the last day of chest radiation is the most important predictor of survival in limited-disease smallcell lung cancer. J Clin Oncol 2006;24(7):1057-63.

9. Spiro SG, James LE, Rudd RM, et al. Early compared with late radiotherapy in combined modality treatment for limited disease small-cell lung cancer: a London Lung Cancer Group multicenter randomized clinical trial and meta-analysis. J Clin Oncol 2006;24(24):3823-30.

10. Bentzen SM, Thames HD. Clinical evidence for tumor clonogen regeneration: interpretations of the data. Radiother Oncol 1991;22(3):161-6.

11. Withers HR, Taylor JM, Maciejewski B. The hazard of accelerated tumor clonogen repopulation during radiotherapy. Acta Oncol 1988;27(2):131-46.

12. Harari PM, Mehta MP, Ritter MA, Petereit DG. Clinical promise tempered by reality in the delivery of combined chemoradiation for common solid tumors. Semin Radiat Oncol 2003;13(1):3-12.

13. Pijls-Johannesma MCG, De Ruysscher DKM, Rutten I, Vansteenkiste JF, Lambin P. Early versus late chest radiotherapy for limited stage small cell lung cancer (Cochrane review). The Cochrane Library (issue 1) 2005.

14. Davis AJ, Tannock JF. Repopulation of tumour cells between cycles of chemotherapy: a neglected factor. Lancet Oncol 2000;1:86-93.

15. Davis AJ, Tannock IF. Tumor physiology and resistance to chemotherapy: repopulation and drug penetration. Cancer Treat Res 2002;112:1-26.

16. Wu L, Tannock IF. Repopulation in murine breast tumors during and after sequential treatments with cyclophosphamide and 5-fluorouracil. Cancer Res 2003;63(9):2134-8.

17. Work E, Nielsen OS, Bentzen SM, Fode K, Palshof T. Randomized study of initial versus late chest irradiation combined with chemotherapy in limited-stage small-cell lung cancer. Aarhus Lung Cancer Group. J Clin Oncol 1997;15(9):3030-7.

18. Jeremic B, Shibamoto $\mathrm{Y}$, Acimovic L, Milisavljevic S. Initial versus delayed accelerated hyperfractionated radiation therapy and concurrent chemotherapy in limited small-cell lung cancer: a randomized study. J Clin Oncol 1997;15(3):893-900.

19. Perry MC, Herndon JE, 3rd, Eaton WL, Green MR. Thoracic radiation therapy added to chemotherapy for small-cell lung cancer: an update of Cancer and Leukemia Group B Study 8083. J Clin Oncol 1998;16(7):2466-7.

20. Skarlos DV, Samantas E, Briassoulis E, et al. Randomized comparison of early versus late hyperfractionated thoracic irradiation concurrently with chemotherapy in limited disease small-cell lung cancer: a randomized phase II study of the Hellenic Cooperative Oncology Group (HeCOG). Ann Oncol 2001;12(9):1231-8. 
21. Murray N, Coy P, Pater JL, et al. Importance of timing for thoracic irradiation in the combined modality treatment of limited-stage small-cell lung cancer. The National Cancer Institute of Canada Clinical Trials Group. J Clin Oncol 1993;11(2):336-44.

22. Takada M, Fukuoka M, Kawahara M, et al. Phase III study of concurrent versus sequential thoracic radiotherapy in combination with cisplatin and etoposide for limited-stage small-cell lung cancer: results of the Japan Clinical Oncology Group Study 9104. J Clin Oncol 2002;20(14):3054-60.

23. Gregor A, Drings $P$, Burghouts $\mathrm{J}$, et al. Randomized trial of alternating versus sequential radiotherapy/chemotherapy in limited-disease patients with small-cell lung cancer: a European Organization for Research and Treatment of Cancer Lung Cancer Cooperative Group Study. J Clin Oncol 1997;15(8):2840-9.

24. Bonner JA, Sloan JA, Shanahan TG, et al. Phase III comparison of twice-daily split-course irradiation versus once-daily irradiation for patients with limited stage small-cell lung carcinoma. J Clin Oncol 1999;17(9):2681-91.

25. Lebeau B, Urban $\mathrm{T}$, Brechot JM, et al. A randomized clinical trial comparing concurrent and alternating thoracic irradiation for patients with limited small cell lung carcinoma. "Petites Cellules" Group. Cancer 1999;86(8):1480-7.

26. Turrisi AT, 3rd, Kim K, Blum R, et al. Twice-daily compared with once-daily thoracic radiotherapy in limited small-cell lung cancer treated concurrently with cisplatin and etoposide. $\mathrm{N}$ Engl $\mathrm{J}$ Med 1999;340(4): 265-71.

27. Turrisi AT, Sherman CA. The treatment of limited small cell lung cancer: a report of the progress made and future prospects. Eur J Cancer 2002;38(2):279-91. 



\section{CHAPTER}

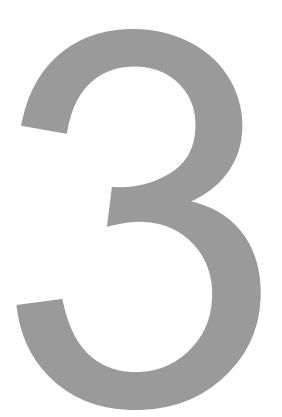

Time between the first day of chemotherapy and the last day of chest radiation is the most important predictor of survival in limited-disease small-cell lung cancer

Journal of Clinical Oncology (2007), 24:1057-1063

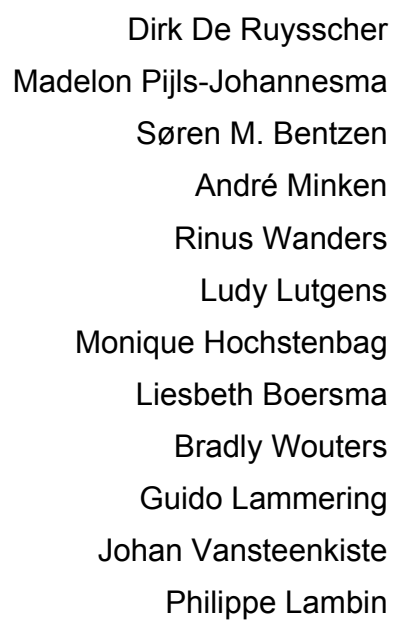




\section{ABSTRACT}

\section{Purpose}

To identify time factors for combined chemotherapy and radiotherapy predictive for long-term survival of patients with limited-disease small-cell lung cancer (LD-SCLC).

\section{Methods}

A systematic overview identified suitable phase III trials. Using meta-analysis methodology to compare results within trials, the influence of the timing of chest radiation and the start of any treatment until the end of radiotherapy (SER) on local tumor control, survival, and esophagitis was analyzed. For comparison between studies, the equivalent radiation dose in 2-Gy fractions, corrected for the overall treatment time of chest radiotherapy, was analyzed.

\section{Results}

The SER was the most important predictor of outcome. There was a significantly higher 5-year survival rate in the shorter SER arms (relative risk [RR] $0.62 ; 95 \% \mathrm{Cl}$, 0.49 to $0.80 ; P$.0003), which was more than $20 \%$ when the SER was less than 30 days (upper bound of $95 \% \mathrm{Cl}, 90$ days). A low SER was associated with a higher incidence of severe esophagitis ( $R R=0.55 ; 95 \% \mathrm{Cl}, 0.42$ to $073 ; P=.0001$ ). Each week of extension of the SER beyond that of the study arm with the shortest SER resulted in an overall absolute decrease in the 5-year survival rate of $1.83 \%-0.18 \%$ $(95 \% \mathrm{Cl})$.

\section{Conclusion}

A short time between the first day of chemotherapy and the last day of chest radiotherapy is associated with improved survival in LD-SCLC patients. The novel parameter SER, which takes into account accelerated proliferation of tumor clonogens during both radiotherapy and chemotherapy, may facilitate a more rational design of combined-modality treatment in rapidly proliferating tumors. 


\section{INTRODUCTION}

Small cell lung cancer (SCLC) accounts for about $20 \%$ of all lung cancer cases, with only one third of the patients presenting with limited disease (LD-SCLC) $)^{1,2}$. Without treatment, tumor progression in patients with SCLC is rapid, with a median survival of 2 to 4 months. Although chemotherapy and the combination of chemotherapy and chest radiotherapy have improved the prognosis substantially, long-term survival remains poor ${ }^{1-7}$. The optimal combination of chemotherapy and chest radiation remains controversial ${ }^{5-7}$.

Accelerated proliferation of tumor clonogens during radiotherapy has been shown to affect the outcome in squamous cell carcinoma of the head and neck both in literature overviews $s^{8,9}$ as well as in randomized controlled trials ${ }^{10,11}$. Although the evidence is not as strong for a number of other solid tumors there are good reasons to believe that accelerated proliferation is an universal response to fractionated radiotherapy ${ }^{12}$. The possibility that cytotoxic chemotherapy also induces accelerated repopulation has attracted less attention ${ }^{13,14}$. However, several studies in murine tumor models show that chemotherapy may induce accelerated repopulation after a delay of 0 to 14 days ${ }^{15-18}$. Clinically, a few studies have shown an increase in cellular proliferation after chemotherapy ${ }^{19,20}$, at least in some patients ${ }^{21}$.

All of these observations suggest that the overall duration of the 'radiochemotherapy package deal' is the most relevant for outcome and this has specifically been proposed for LD-SCLC due to the natural history of this disease ${ }^{7,14}$.

We propose to use the time from the start of any treatment to the end of chest irradiation (SER) as a quantitative measure reflecting proliferation of cells in the primary tumor. This is based on two assumptions: (1) the first cytotoxic insult (whether it is from chemotherapy or radiotherapy) will trigger accelerated tumor repopulation; (2) bulky disease must be controlled by the end of radiotherapy, or the treatment will fail in nearly all patients.

Here, we test the prognostic value of the SER in an analysis of outcome data from six randomized controlled trials of various combinations of chemotherapy and radiotherapy in patients with LD-SCLC.

\section{METHODS}

\section{Identification of studies}

The data were based on a recent systematic review and meta-analysis from our group in the Cochrane Database of Systematic Reviews ${ }^{22}$. Briefly, eligible studies were randomized controlled clinical trials, fully published in journals or identified from 
other sources (abstracts and proceedings of relevant scientific meetings, and contacts with investigators) for which full details were available from investigators. Patients of any age had to have histologically or cytologically proven LD-SCLC and a performance status 0-2. We used the following definition of 'limited disease': cancer confined to one hemi-thorax including contralateral mediastinal and hilar lymph nodes as well as ipsilateral and/or bilateral supraclavicular involvement, but excluding malignant pleural effusion.

A search for studies was undertaken in the following electronic databases: the Cochrane Central Register of Controlled Trials (CENTRAL, 2003 Issue 4), MEDLINE (1966 to present), EMBASE (1974 to present), CINAHL (1982 to present). Furthermore, the Cochrane Lung Cancer Groups Specialized Register was searched. Searches were performed without language restrictions. Reference lists from identified studies were scrutinized for references to any additional studies. The electronic searches for clinical trials were complemented with manual searches of the following oncology journals: International Journal of Radiation, Oncology, Biology and Physics (1985-present); Radiotherapy and Oncology (1985-present); Journal of Clinical Oncology (1985-present); Clinical Oncology (1999-present); Lung Cancer (1985present); and Thorax (1985-present). Abstracts from the principal oncology conferences from 1985 and onwards with a minimum follow up of 3 years were hand searched. Colleagues, collaborators, and other experts in the field were asked to identify missing and unreported trials.

\section{Parameters}

A priori, we identified the following parameters to be analyzed for their influence on local tumor control, survival, esophagitis and pneumonitis: the total radiotherapy dose, the overall treatment time of chest radiotherapy, the day of start of radiotherapy as an indicator of early versus late radiation, the SER as defined above, concurrent versus sequential radiotherapy and chemotherapy and the Equivalent Radiation Dose in 2-Gy fractions, corrected for the overall treatment time of chest radiotherapy $\left(E Q D_{2, T}\right)$.

As individual patient data were not available, we calculated the SER from the published data of each original paper. In case the start of radiotherapy was not required at a specific day, we took the mean between the permitted time limits in the study protocol. This was the case in the trial of Turrisi et $\mathrm{al}^{23}$, where the start of chest radiotherapy was allowed during the first cycle of chemotherapy, i.e. between days 1 and 20. The start of chest radiotherapy was thus assumed to be day 10 for this study. 
For local tumor control comparisons, we omitted the trial of Takada et $\mathrm{al}^{24}$ because only data on the first site of recurrence were available, whereas in all other studies cumulative local tumor control rates were provided.

A quantitative estimate of a 1-week increase in SER was obtained from the overall odds ratio between the two arms of a specific trial. The change in the log odds ratio per week was assumed to be constant, when calculating this quantity.

As a measure of the intensity of chest radiotherapy, we used the equivalent dose in 2-Gy fractions with correction for overall treatment time, $E Q D_{2, T}$, which was calculated in two steps ${ }^{25}$. First, an adjustment for dose per fraction was made:

$E Q D_{2}=D \cdot \frac{d+\alpha / \beta}{2+\alpha / \beta}$

where $\mathrm{D}$ is the total radiation dose, $\mathrm{d}$ the dose per fraction and $\alpha / \beta=10 \mathrm{~Gy}$.

To take into account proliferation of tumor cells, we used the formula

$E Q D_{2, T}=E Q D_{2}-\operatorname{MAX}\left(\left(T-T_{K}\right), 0\right) . D_{\text {prolif }}$

where $T_{K}$ is the kick-in time of accelerated repopulation, assumed in our case to be 30 days. The function $\operatorname{MAX}\left(\left(T-T_{K}\right), 0\right)$ will have the value of $\mathrm{T}-\mathrm{T}_{K}$ if this difference is positive, and zero in all other cases. The parameter $D_{\text {prolif }}$ is the dose recovered per day. This parameter is not well estimated for SCLC, but we assumed that the dose lost per day was at least that estimated for squamous cell carcinoma of the head and neck and we therefore assumed $D_{\text {prolif }}=0.7$ Gy per day ${ }^{25}$.

Possible incomplete repair between multiple fractions per day was not considered as there are no reliable data for SCLC.

\section{Chemotherapy}

Two types of chemotherapy were used in the studies: platinum-based or anthracyclin-based. In only one trial, a non-platinum-based chemotherapy regimen was administered concurrently with chest irradiation ${ }^{26,27}$. In all other studies, platinumetoposide was given during chest radiotherapy ${ }^{23,24,28-32}$.

\section{Statistics}

A weighted estimate of the typical treatment effect across studies was computed for the 5-year survival data and the SER. The relative risk (RR) was used as the effect 
measure. Possible censoring of survival data could not be taken into account as not enough information could be derived from the published data to calculate this. Chisquare heterogeneity tests were used to test for statistical heterogeneity among trials. As we anticipated that the trial results would be heterogeneous, all analyses were performed using a random effects model. Two-tailed P-values of $<0.05$ were considered to be significant. Dose-response relationships were analyzed using logistic regression.

All statistical analysis were performed with RevMan (Review Manager (RevMan) [Computer program], Version 4.2 for Windows. Oxford, England: The Cochrane Collaboration, 2003.) and STATA version 8.0 for Windows.

\section{RESULTS}

\section{Identification of phase III trials}

Eight phase III trials with the same chemotherapy regimen in each arm, comparing two different radiation schedules and reporting long-term survival data were identified $23,24,26-32$. Because in two major trials ${ }^{23,28}$, the difference in survival between radiation schedules only became apparent after 2 years or more, we only analyzed 5-year survival data from these phase III studies. Five-year survival data are also very likely not meaningfully influenced by the use of second line chemotherapy, whereas 2-year data may be. The trial of Bonner et $\mathrm{al}^{32}$ was omitted because the time factors in both arms of the trial were the same and because patients were only included when they had a remission after induction chemotherapy. Therefore, comparison with the other studies where all patients were included upfront was not possible. The EORTC trial of Gregor et $\mathrm{al}^{30}$ was not included as no 5-year survival data were available.

One trial used doxorubicin-based chemotherapy concomitantly with chest radiotherapy ${ }^{26,27}$. This type of chemotherapy is currently not used, because of its lower efficacy $^{33}$ and higher toxicity ${ }^{34}$ in comparison with platinum-based chemotherapy. The trial of Work et $\mathrm{al}^{29}$ was omitted because no concurrent chemotherapy and chest radiation was used, which is at present considered to be the standard therapy. We therefore only analyzed the four trials where platinum combined with etoposide was administered during chest radiotherapy ${ }^{23,24,28,31}$, and for which 5 -year survival data were available. In total, the 4 studies comprised 1,056 patients. Table 1 summarizes the main features of the included trials. 


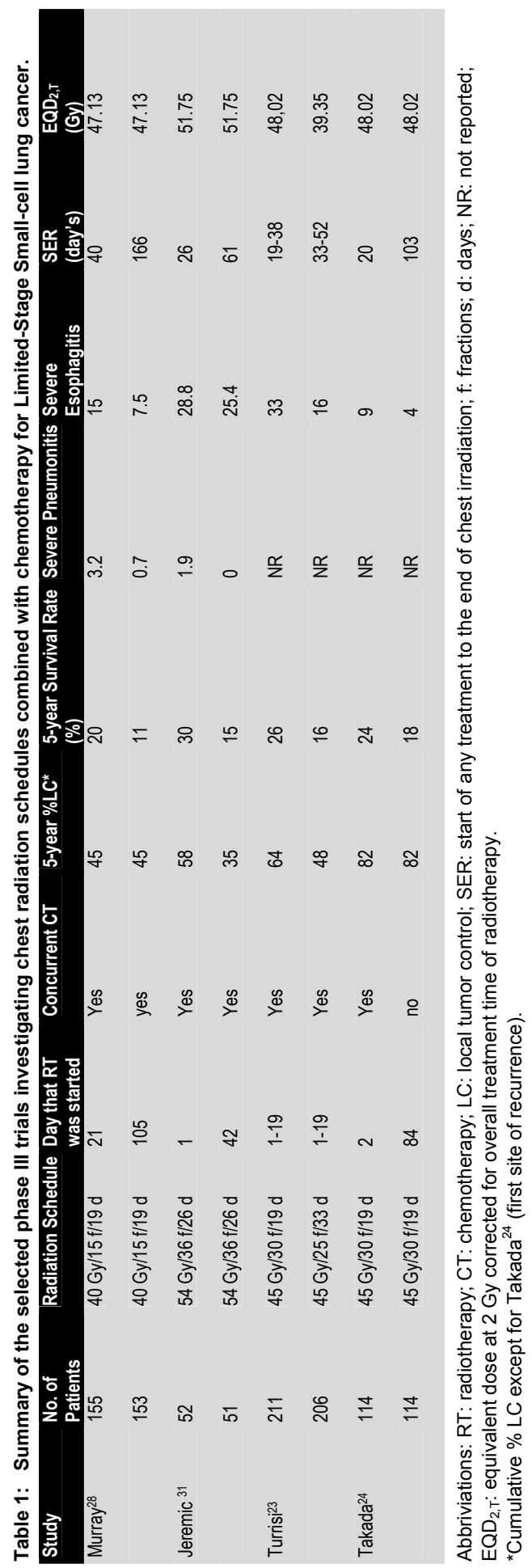


Of these four trials, the study of Takada et $\mathrm{al}^{24}$ only provided data on the first site of tumor recurrence. In the other trials, the cumulative proportion of local tumor recurrence was given ${ }^{23,28,29,31}$. We therefore included only the latter studies for this analysis.

Only two trials used radiopneumonitis as an endpoint ${ }^{28,31}$. In the study of Turrisi et al ${ }^{23}$, radiopneumonitis was not specifically mentioned, only "pulmonary effects", which may include other types of lung injury. The meta-analysis technique could therefore not be applied for radiopneumonitis.

The total radiation dose, expressed as nominal doses or as $E Q D_{2}$, differed only slightly between the studies and no trials used different total radiation doses between the study arms. This parameter was therefore not investigated as a variable in the comparisons within trials.

As the overall treatment time of thoracic radiotherapy was identical in all study arms, except in one trial ${ }^{23}$, this variable could not be investigated using meta-analysis techniques.

The timing of radiotherapy was different in three studies ${ }^{24,28,31}$. Meta-analysis techniques could thus be used.

Only one trial ${ }^{24}$ compared concurrent or sequential chemotherapy and radiotherapy, and therefore the potential importance of the sequencing of chemotherapy and radiotherapy could not be tested using meta-analysis techniques.

\section{Analysis}

\section{Comparisons within trials}

Comparisons were calculated using meta-analysis techniques.

Two parameters were predictive for the 5-year survival: the SER and the timing of chest radiotherapy, the SER being the strongest predictor. The 5-year survival was highest in the shorter SER arms (RR:0.62; 95\% Cl: 0.49-0.80; $p=0.0003$ ) or when chest radiotherapy was delivered early after the start of chemotherapy compared to late (RR:0.63; 95\% Cl: 0.45-0.88; $p=0.007$ ) (figures $1 \mathrm{~A}$ and $1 \mathrm{~B}$ ).

Neither the SER (RR:0.81; 95\% Cl: 0.64-1.02; $p=0.07$ ) nor the timing of chest radiation (RR:0.83; $95 \% \mathrm{Cl}: 0.55-1.27 ; p=0.39$ ) were predictive for local tumor control (figures $2 \mathrm{~A}$ and $2 \mathrm{~B})$. 


\section{A}

Review: Early versus Late Chest Radiotherapy in patients with limited stage small cell lung cancer (SER)

Comparison: $\quad 02$ High SER VS LOW SER

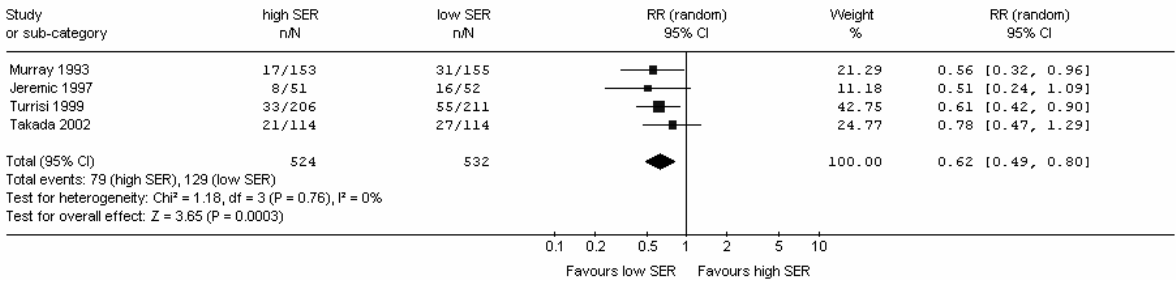

\section{B}

Review: $\quad$ Early versus Late Chest Radiotherapy in patients with limited stage small cell lung cancer (SER) Comparison: 01 Early versus Late Chest RT

Outcome: $\quad 035$-yrs Survival

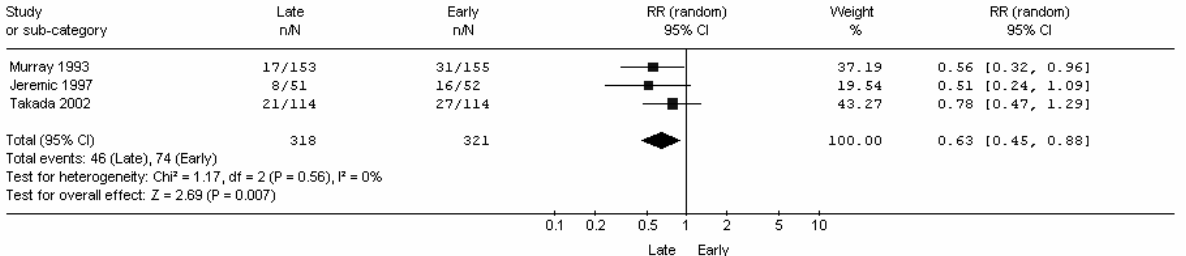

Figure 1 (A) The survival at 5 years as a function of the time from Start of any treatment and the End of Radiotherapy (SER).The RR for the 5-year survival is significantly in favor of the study arms with the lowest SER ( $p=0.0003)$. (B) The survival at 5 years as a function of the timing of the chest radiotherapy. The RR for the 5 -year survival is significantly in favor of the study arms with early radiotherapy $(p=0.007)$. 
A

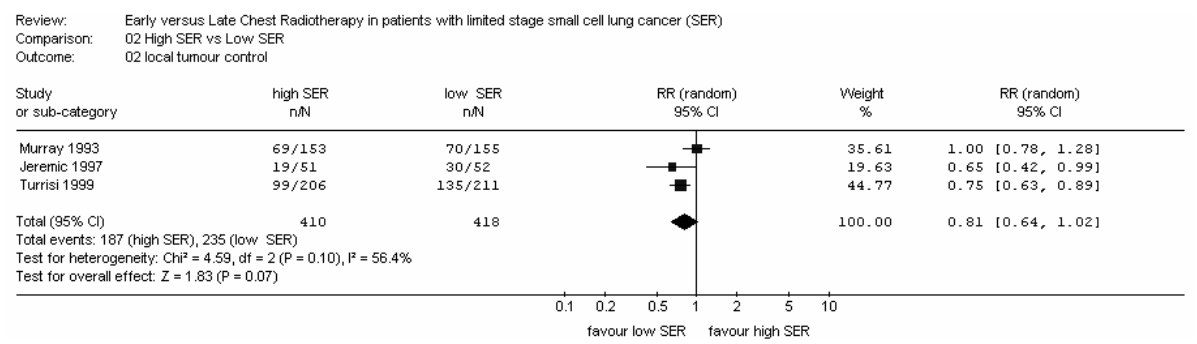

\section{B}

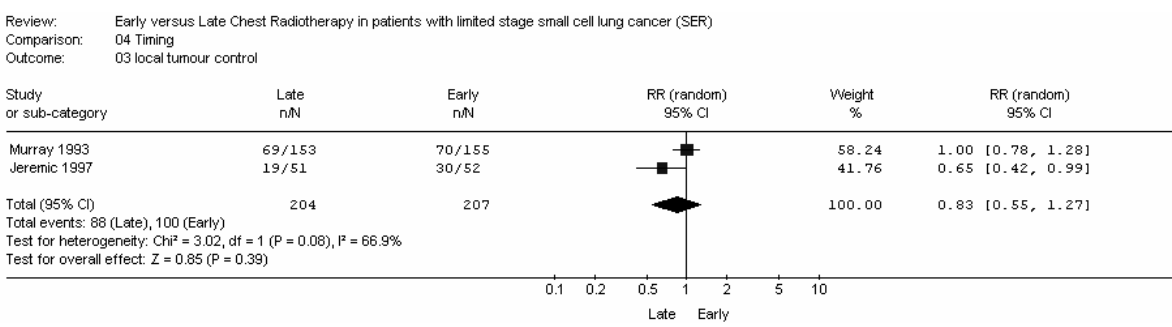

Figure 2 (A) Local tumor control at 5 years as a function of the time from Start of any treatment and the End of Radiotherapy (SER). The RR for the local tumor control is not significantly different between study arms with a low or a high SER $(p=0.07)$. (B) Local tumor control at 5 years as a function of the timing of chest radiotherapy. The RR for the local tumor control is not significantly different between study arms with early or late chest radiotherapy $(p=0.39)$.

Both a short SER and early chest radiotherapy were associated with a higher incidence of severe esophagitis (RR:0.55; 95\% Cl: 0.42-0.73; $p<0.0001$; RR:0.63; 95\% Cl: 0.40-1.00; $p=0.05$; respectively) (figures $3 \mathrm{~A}$ and $3 \mathrm{~B}$ ).

Each week of extension of the SER beyond that of the study arm with the shortest SER, resulted in a decrease of the 5-year survival with an overall RR of 0.96 (95\% Cl:0.94-0.98; $p<0.0001$ ) (figure 4).

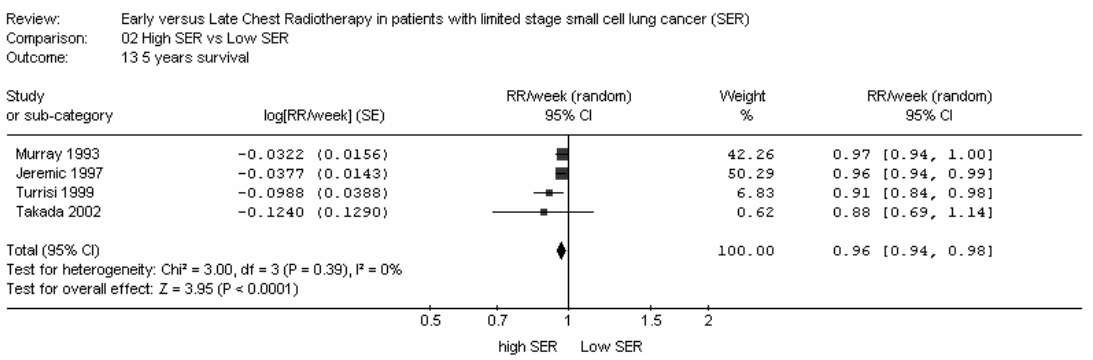

Figure 4 Relative Risk (RR) of the 5-year survival per week extension of the time from the Start of any treatment and the End of Radiotherapy (SER; comparison within trials: arm 2 - arm 1). 


\section{A}

Review: Early versus Late Chest Radiotherapy in patients with linited stage small cell lung cancer (SER) Comparison: $\quad 02$ High SER vs Low SER Outcome: $\quad 04$ severe oesophagitis

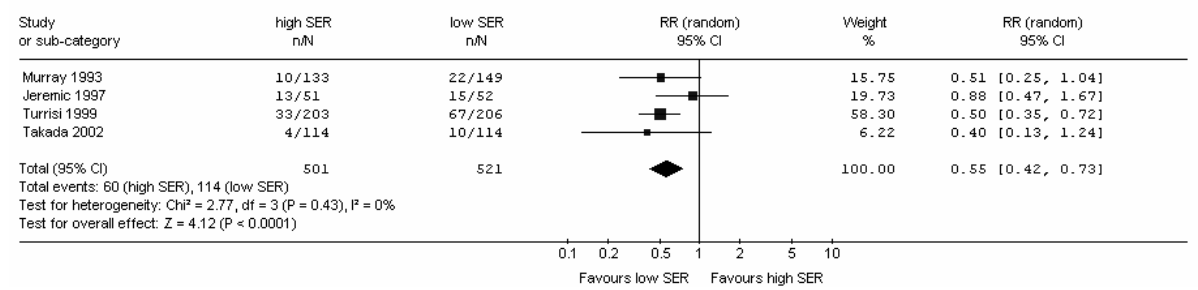

\section{B}

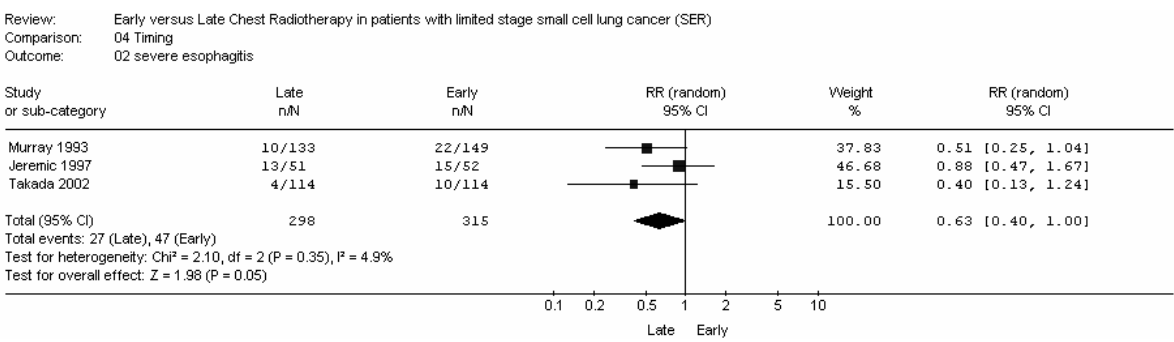

Figure 3 (A) Severe esophagitis as a function of the time from Start of any treatment and the End of Radiotherapy (SER). The RR is significantly different between study arms with a low or a high SER ( $<<0.0001)$, with a low SER associated with a higher incidence of severe esophagitis. (B) Severe esophagitis as a function of the timing of chest radiotherapy.The $R R$ is significantly different between study arms with early or late radiation $(p=0.03)$, with early radiation associated with a higher incidence of severe esophagitis.

\section{Comparisons between trials}

The 5 -year survival was more than $20 \%$ when the SER was less than 30 days (upper bound $95 \% \mathrm{Cl}$ : 90 days) (figure 5).

No significant correlation between the local tumor control and the $E_{2, T}(p=0.76)$ was found (figure 6).

\section{DICUSSION}

Accelerated proliferation of tumor clonogens is a well-recognized cause of failure after radiotherapy and chemotherapy in several malignancies ${ }^{8-22}$. These considerations stimulated us to look for time factors reflecting both chemotherapy and radiotherapy associated accelerated proliferation of tumor clonogens. Such factors could facilitate a more rational design of combined modality treatment optimizing anti-tumor effect and normal tissue sparing. We chose to investigate LD-SCLC, because this is a rapidly growing cancer where both chemotherapy and chest radiotherapy play a role ${ }^{1-7}$. 


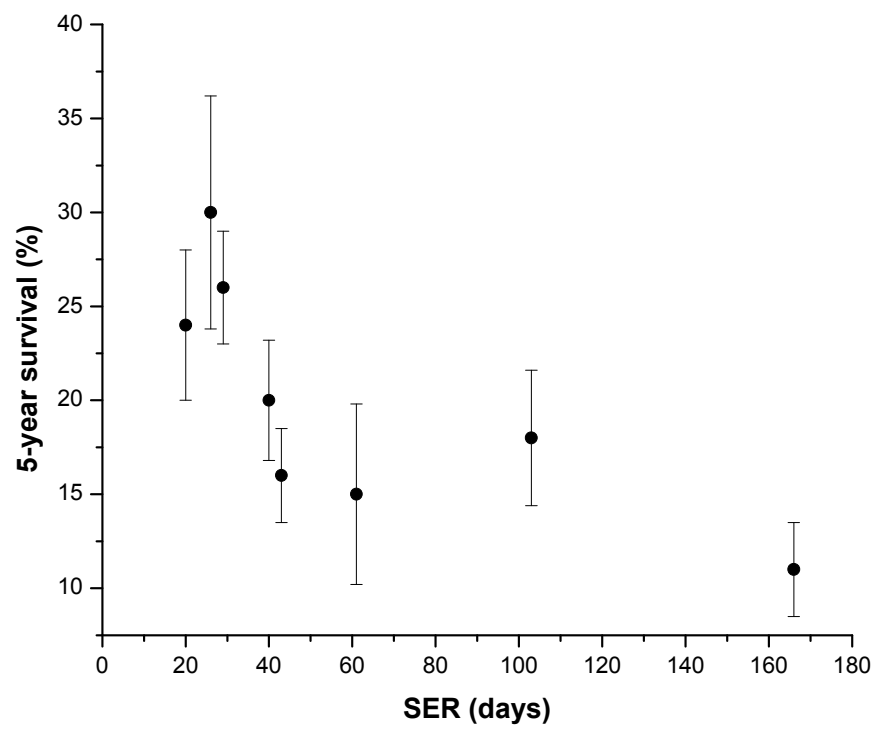

Figure 5 The survival at 5 years as a function of the time from S⿱ therapy (SER). Each dot represents a single trial \pm S.E.

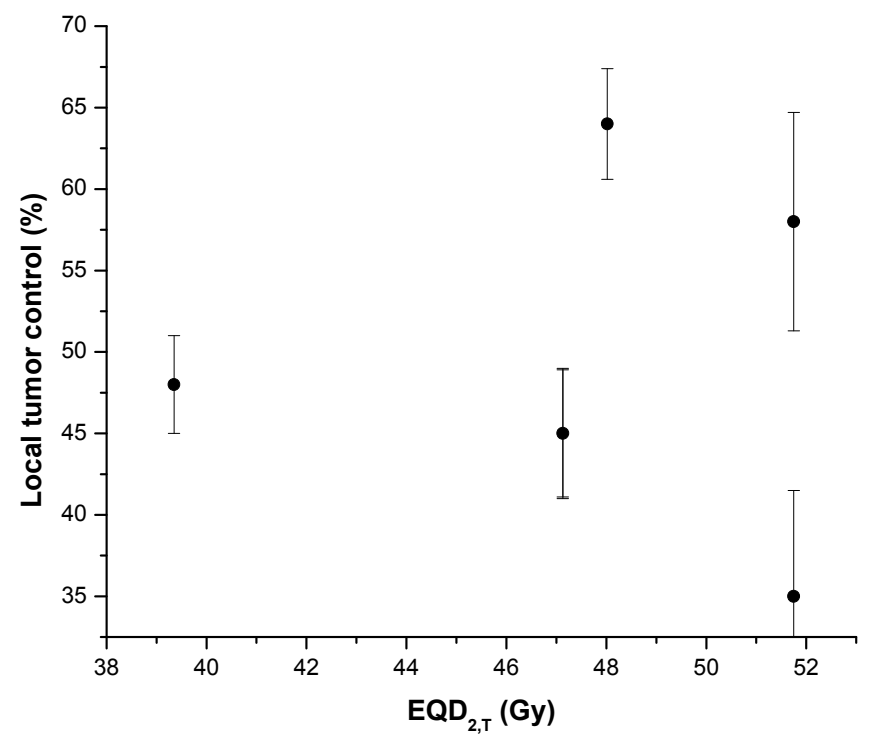

Figure 6 Local Tumor Control as a function of the Equivalence Dose at $2 \mathrm{~Gy}$, corrected for the overall treatment time of chest radiotherapy $\left(E Q D_{2, T}\right)$. Each dot represents a single trial \pm S.E. $(p=0.76)$. 
We decided to include only trials where platinum-based chemotherapy was delivered concurrently with chest radiotherapy, as this is at present the standard treatment $23,24,28,29,31$. Only five-year survival data were considered, as the difference in survival between radiation schedules only became apparent after 2 years or more in two major trials ${ }^{23,28}$. Moreover, this also avoided possible interference with second line chemotherapy. Trials in which there was no difference in time factors between the arms were omitted. Prophylactic cranial irradiation (PCl) was given to at least a group of patients in all studies and it was therefore not possible to stratify for this parameter in the analysis.

We used the time from the start of any treatment to the end of chest irradiation (SER) as a quantitative measure reflecting proliferation of cells in the primary tumor.

In order to avoid the biases of comparing results between trials, we first analyzed the results within studies. Both the SER and the timing of chest radiotherapy correlated significantly with the long-term survival, the latter finding being in agreement with two recent meta-analyses ${ }^{22,35}$. Although early chest radiation was correlated with a short SER in all ${ }^{24,28,29,31}$, but one ${ }^{23}$ study, the SER, a parameter that takes into account time factors of both radiotherapy and chemotherapy, correlated more strongly with the long-term survival than the timing of radiation. From a biological point of view, the SER is a logical approach to investigate the integration of radiotherapy and drugs as it considers not only whether a drug was administered concurrently or early during radiation or not, but also the time interactions when agents are delivered intermittently at days when no radiotherapy is given, or even when drugs are administered before the start of radiotherapy. This may be increasingly important when targeted agents are used ${ }^{36}$.

In order to quantify the effect of a change in SER on the 5-year survival, we estimated that each week of extension of the SER beyond that of the study arm with the shortest SER, resulted in an absolute decrease of the 5-year survival of $1.83 \%$ corresponding to an overall $\mathrm{RR}$ per week of 0.96 (95\% Cl:0.94-0.98; $\mathrm{p}<0.0001)$. There are two caveats here. First of all, these estimates are strongly influenced by a single trial, that of Turrisi et $\mathrm{al}^{23}$. Secondly, treatment is continued in the long-SER arm during the period when SER is estimated. Thus, the effect of an increased SER may depend on the exact temporal distribution of further therapy as well as, of course, on its effectiveness. The simple interpretation of the SER estimate requires that therapeutic effects of cycles of chemotherapy and fractions of radiotherapy are nonoverlapping and independent.

The highly significant relation between the SER and the 5-year survival supports the occurrence of accelerated proliferation of tumor clonogens during chemotherapy and radiotherapy. As a consequence, the best survival of patients with fast proliferating tumors may be achieved when 2 or 3 full-dose cycles of chemotherapy and chest radiotherapy are delivered before accelerated tumor cell proliferation starts, whether 
triggered by chemotherapy or by chest radiation. Of course, this has to be balanced with acute side-effects which also occur more frequently in schedules with a short SER. Indeed, a short SER or the early delivery of chest radiotherapy were associated with a higher incidence of severe esophagitis. This is in line with previous findings, indicating that intensifying chemotherapy and radiotherapy regimen increase the cytotoxic effects on rapidly proliferating cells, including the mucosa ${ }^{11,12}$. As too few studies used pneumonitis as an endpoint, no meta-analysis could be performed for this side-effect.

No significant relation between the SER, the timing of chest radiotherapy or the $E Q D_{2, T}$ and the local tumor control was found. This may be explained by the inadequate assessment of local tumor control ${ }^{7}$.

When comparing the results between trials, which is obviously fraught with errors, we found that a 5 -year survival of more than $20 \%$ could be achieved when the SER was less than 30 days, with an upper bound of the $95 \% \mathrm{Cl}$ of 90 days. In the absence of for that purpose designed randomized trials, the exact value of the optimal SER thus remains uncertain.

Our results are in line with an earlier trial ${ }^{37}$ in which non-platinum chemotherapy was administered that concluded that the optimal treatment of patients with LD-SCLC was concurrent chemotherapy and chest radiation given in 3-4 weeks.

We conclude that the present results introduce the SER as a novel parameter that could make rational design of trials combining chemotherapy and radiotherapy more feasible. However, confirmation of the value of the SER should be done in prospective studies or by a meta-analysis with individual patient data from previous phase III trials. 


\section{REFERENCES}

1. Bunn PA Jr, Carney DN. Overview of chemotherapy for small cell lung cancer. Semin Oncol 24:S69S74, 1997

2. Stupp R, Monnerat C, Turrisi AT, et al. Small cell lung cancer: state of the art and future perspectives. Lung Cancer 45:105-117, 2004

3. Kelly K. New chemotherapy agents for small cell lung cancer. Chest 117:S156-S162, 2000

4. Pignon JP, Arriagada R, Ihde DC, et al. A meta-analysis of thoracic radiotherapy for small-cell lung cancer. N Engl J Med 327:1618-1624, 1992

5. Warde $P$, Payne D. Does thoracic irradiation improve survival and local control in limited-stage smallcell carcinoma of the lung? A meta-analysis. J Clin Oncol 10:890-895, 1992

6. Kumar $P$. The role of thoracic radiotherapy in the management of limited-stage small cell lung cancer: past, present, and future. Chest 112:S259-S265, 1997

7. De Ruysscher D, Vansteenkiste J. Chest radiotherapy in limited-stage small cell lung cancer: facts, questions, prospects. Radiother Oncol 55:1-9, 2000

8. Withers HR, Taylor JM, Maczejewski B. The hazard of accelerated tumor clono gen repopulation during radiotherapy. Acta Oncol 27: 131-146, 1998

9. Bentzen SM, Thames HD. Clinical evidence of tumor clonogen regeneration: in terpretations of the data. Radiother Oncol 22: 161-166, 1991

10. Overgaard J, Hansen HS, Specht L, et al. Five compared with six fractions per week of conventional radiotherapy of squamous-cell carcinoma of head and neck: DAHANCA 6 and 7 randomised controlled trial. Lancet 362:933-940, 2003 Erratum in: Lancet 362:1588, 2003

11. Bernier J, Bentzen SM. Altered fractionation and combined radio-chemotherapy approaches: pioneering new opportunities in head and neck oncology.

Eur J Cancer 39:560-571, 2003

12. Bentzen SM. Repopulation in radiation oncology: perspectives of clinical research. Int $J$ Radiat Biol 79:581-585, 2003

13. Davis, A. J., and Tannock, I. F. . Repopulation of tumor cells between cycles of chemotherapy: a neglected factor. Lancet Oncol 1:86-93, 2000

14. Davis AJ, Tannock IF. Tumor physiology and resistance to chemotherapy: repopulation and drug penetration. Cancer Treat Res 112:1-26, 2002

15. Wu L, Tannock IF. Repopulation in Murine Breast Tumors during and after Sequential Treatments with Cyclophosphamide and 5-Fluorouracil. Cancer Res 63: 2134-2138, 2003

16. Stephens TC, Peacock JH. Tumour volume response, initial cell kill and cellular repopulation in B16 melanoma treated with cyclophosphamide and 1-(2-chloroethyl)-3-cyclohexyl-1-nitrosourea. Br J Cancer 36:313-321, 1977

17. Rosenblum ML, Knebel KD, Vasquez DA, et al. In vivo clonogenic tumor cell kinetics following 1,3bis(2-chloroethyl)-1-nitrosourea brain tumor therapy. Cancer Res 36:3718-3725, 1976

18. Milas L, Nakayama T, Hunter N, et al. Dynamics of tumor cell clonogen repopulation in a murine sarcoma treated with cyclophosphamide. Radiother Oncol 30:247-253, 1994

19. Budach W, Paulsen F, Welz S, et al. Mitomycin C in combination with radiotherapy as a potent inhibitor of tumor cell repopulation in a human squamous cell carcinoma. $\mathrm{Br} \mathrm{J}$ Cancer 86: 470-476, 2002

20. Bourhis J, Bosq J, Wilson GD, et al. Correlation between p53 gene expression and tumor-cell proliferation in oropharyngeal cancer. Int J Cancer 57:458-462, 1994

21. Nijman HW, Kenemans P, Poort-Keesom RJ, et al. Influence of chemotherapy on the expression of p53, HER-2/neu and proliferation markers in ovarian cancer. Eur J Obstet Gynecol Reprod Biol 83:201206, 1999

22. Pijls-Johannesma MCG, De Ruysscher DKM, Rutten I, et al. Early versus late chest radiotherapy for limited stage small cell lung cancer. In: The Cochrane Library, Issue 1, 2005. Chichester, UK: John Wiley \& Sons, Ltd. 
23. Turrisi AT, Kim K, Blum R, et al. Twice-daily compared with once-daily thoracic radiotherapy in limited small-cell lung cancer treated concurrently with cisplatin and etoposide. N Engl J Med 340:265-271, 1999

24. Takada M, Fukuoka M, Kawahara M, et al. Phase III study of concurrent versussequential thoracic radiotherapy in combination with cisplatin and etoposide for limited-stage small-cell lung cancer: results of the Japan Clinical Oncology Group Study 9104. J Clin Oncol 20:3054-3060, 2002

25. Hendry JH, Bentzen SM, Dale RG, et al. A modelled comparison of the effects of using different ways to compensate for missed treatment days in radiotherapy. Clin Oncol (R Coll Radiol) 8:297-307, 1996

26. Perry MC, Eaton WL, Propert KJ, et al. Chemotherapy with or without radiation in limited stage smallcell carcinoma of the lung. N Engl J Med 316: 912-918, 1987

27. Perry MC, Herndon JE, Eaton WL, et al. Thoracic radiation therapy added to chemotherapy for smallcell lung cancer: an update of Cancer and Leukemia Group B Study 8083. J Clin Oncol 16:2466-2467, 1998

28. Murray N, Coy P, Pater JL, et al. Importance of timing for thoracic irradiation in the combined modality treatment of limited-stage small-cell lung cancer. The National Cancer Institute of Canada Clinical Trials Group. J Clin Oncol 11:336-344, 1993

29. Work E, Nielsen OS, Bentzen SM, et al. Randomized study of initial versus late chest irradiation combined with chemotherapy in limited-stage small-cell lung cancer. Aarhus Lung Cancer Group. J Clin Oncol 15:3030-3037, 1997

30. Gregor A, Drings P, Burghouts J, et al. Randomized trial of alternating versussequential radiotherapy/chemotherapy in limited-disease patients with small-cell lung cancer: a European Organization for Research and Treatment of Cancer Lung Cancer Cooperative Group Study. J Clin Oncol 15:28402849, 1997

31. Jeremic B, Shibamoto $Y$, Acimovic $L$, et al. Initial versus delayed accelerated hyperfractionated radiation therapy and concurrent chemotherapy in limited small-cell lung cancer: a randomized study. J Clin Oncol 15:893-900, 1997

32. Bonner JA, Sloan JA, Shanahan TG, et al. Phase III comparison of twice-daily split-course irradiation versus once-daily irradiation for patients with limited stage small-cell lung carcinoma. J Clin Oncol 17:2681-2691, 1999

33. Sundstrom S, Bremnes RM, Kaasa S, et al. Cisplatin and etoposide regimen is superior to cyclophosphamide, epirubicin, and vincristine regimen in small-cell lung cancer: results from a randomized phase III trial with 5 years' follow-up. J Clin Oncol 20:4665-4672, 2002

34. Johnson DH, Bass D, Einhorn LH, et al. Combination chemotherapy with orwithout thoracic radiotherapy in limited-stage small-cell lung cancer: a randomized trial of the Southeastern Cancer Study Group. J Clin Oncol 11:1223-1229, 1993

35. Fried DB, Morris DE, Poole C, et al. Systematic review evaluating the timing of thoracic radiation therapy in combined modality therapy for limited-stage small-cell lung cancer. J Clin Oncol 22:4785-93, 2004

36. Moeller BJ, Dreher MR, Rabbani ZN, et al. Pleiotropic effects of HIF-1 blockade on tumor radiosensitivity. Cancer Cell 8:99-110, 2005

37. Catane R, Lichter A, Lee $\mathrm{YJ}$, et al. Small cell lung cancer: analysis of treatment factors contributing to prolonged survival. Cancer 48:1936-43, 1981 


\section{PART 2}

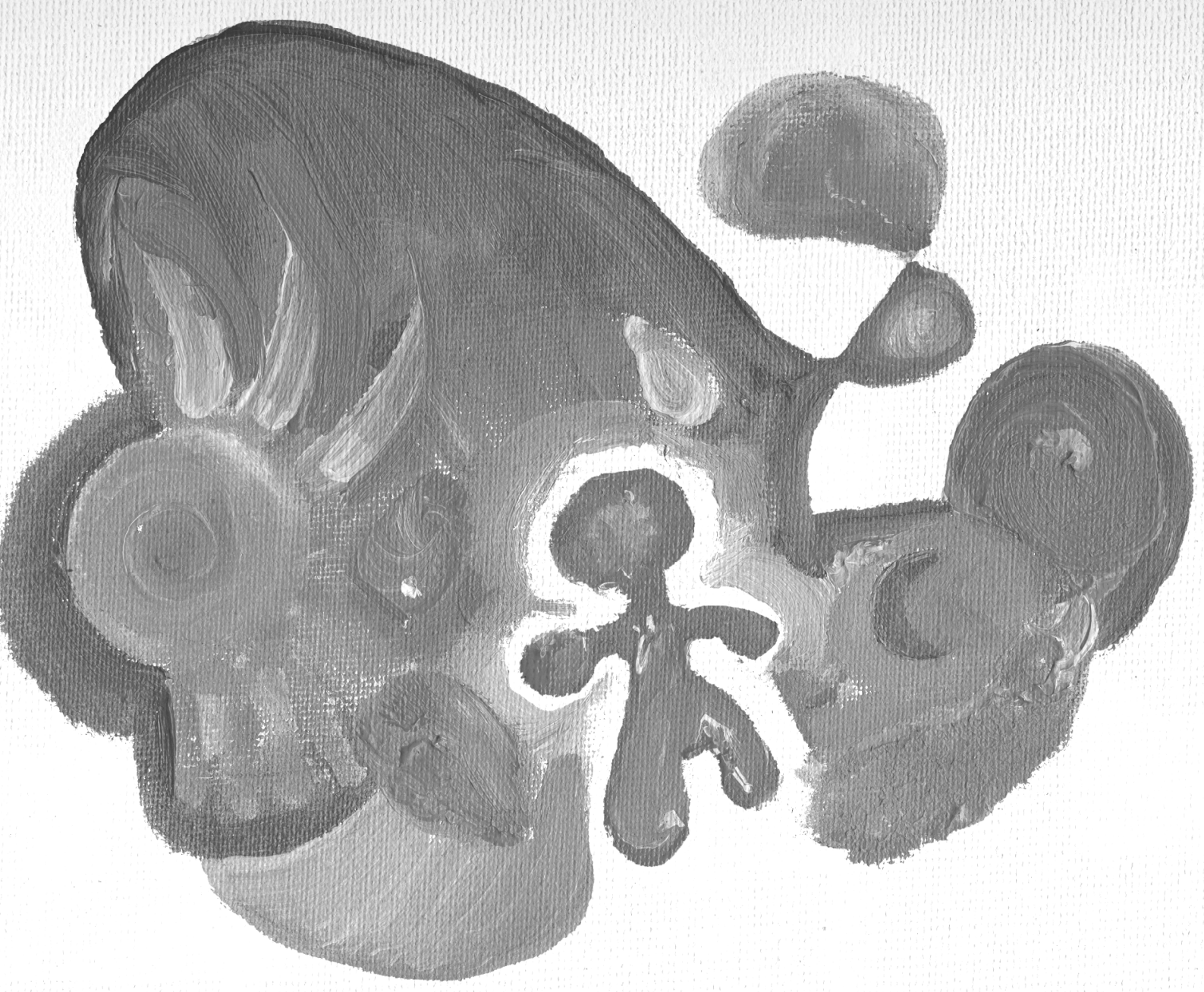




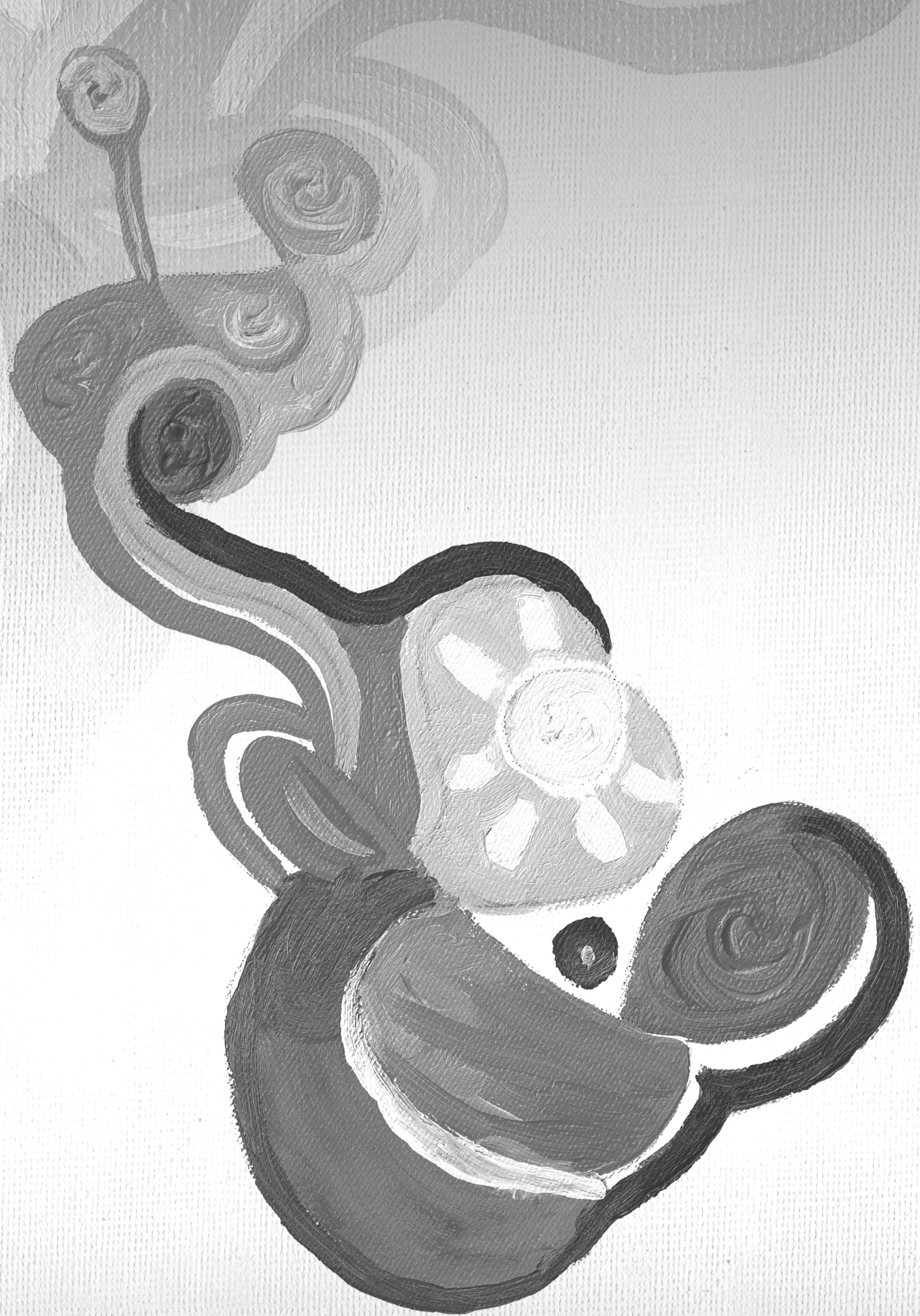




\section{CHAPTER}

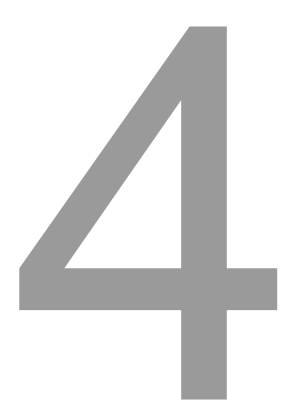

Health related quality of life assessment instruments: A prospective study on preference and acceptability among cancer patients referred for radiotherapy

European Journal of Cancer (2005), 41: 2250-2256

Madelon Pijls-Johannesma Anouk Pijpe Ruud Kempen Philippe Lambin Pieter Dagnelie 


\begin{abstract}
The aim of this study was to determine the preferences for and acceptability of existing Quality of Life (QoL) questionnaires among cancer patients. Sixty-two patients completed a set of questionnaires. Criteria were the percentage of missing values, difficult items and the preferences for the answer options. Results indicated small proportions of missing values and difficult items for the EORTC QLQ-C30, the MFI, RSCL, and the MQ (0.0-1.0\%). Considerably higher proportions of missing values and difficult items were found for the VAS and the PFS (2.5-3.4\%). The answer option of the RSCL subscale activity level was most preferred (29\% of subjects), whereas the VAS and PFS were least preferred (19\% and $21 \%$, respectively). Our results indicate that patients prefer answer categories that have descriptors in words or a narrow range of answer options, rather than non-described answer options or a broad range of answer options per question.
\end{abstract}




\section{INTRODUCTION}

Health related quality of life (HRQoL) can be defined as the level of function and wellbeing with respect to the physical, psychological and social domain ${ }^{1,2}$. HRQoL assessment is not only used to evaluate the effect of clinical and psychosocial interventions, but also as a source of information about the range patients' physical, functional and psychosocial problems that may contribute to an improved physicianpatient communication ${ }^{2}$. Especially in cancer, a disease which has a major impact on patients' lives, the importance of HRQoL assessment is generally accepted.

The usual method of collecting HRQoL information is by self-assessment questionnaires. During the last two decades, a number of these questionnaires have been developed $^{3-7}$. So far, evaluations have mainly focused on psychometric properties of such HRQoL scales and not on the patient's preferences and acceptability of these scales. Preference and acceptability are nevertheless important issues for several reasons. First, the assessment of HRQoL in cancer patients is often hampered by the incompletion of scales, a phenomenon which can be caused by questions that are unclear to patients or questions that are considered as offensive to patients. Secondly, if these instruments are not filled out in the correct manner, this may lead to incorrect conclusions. Third, in the setting of a busy outpatient clinic with limited staff, HRQoL instruments must be self-instructive, easily understandable and reasonably convenient for the patient in terms of time and effort. Recently, a study has been published on patient preference for HRQoL measures, focussed on two specific core measures of QoL (EORTC QLQ-C30 and the FACIT ) ${ }^{8}$, though not on HRQoL measures in general.

The aim of the present study was to determine preferences for and acceptability of a number of popular validated self-assessment HRQoL scales among breast and lung cancer patients referred for radiotherapy with curative intention in an outpatient oncology clinic.

\section{PATIENTS AND METHODS}

\section{Study population}

Eligible patients were those with any stage of breast cancer or lung cancer who had been selected for high-dose radiotherapy ( $\geq 50 \mathrm{~Gy}$ ). Patients, having undergone surgery for lung cancer were excluded. From June 2002 to October 2002, we invited 70 patients, older than 18 years of age and with World Health Organization (WHO) performance status 0-2 to participate ${ }^{9}$. Prior to radiotherapy, patients were asked to complete a set of questionnaires which included a demographic profile and several existing multidimensional validated questionnaires (see below). After completion of 
the set of questionnaires, patients were asked to fill out an evaluation form. The study was approved by the local ethics committee, according to the Dutch legislation, all patients gave written informed consent before the start of the study.

\section{Measurement tool}

The set of questionnaires or scales (which we will call below "The measurement tool"), was composed of a Visual Analogue Scale and five already existing multidimensional validated scales, all of which had previously been used in clinical practice to measure HRQoL and more specifically fatigue as it is one of the most important aspects of HRQoL in cancer patients ${ }^{10-12}$. Therefore we chose to include especially those (sub-)scales in which the concept of fatigue is represented. Since a recent study showed no order effects on the assessment of HRQoL in cancer patients ${ }^{13}$, and more recently a study showed that the order of administration should be consistent throughout the study ${ }^{14}$, all patients received the identical measurement tool, as presented in Table 1.

The Visual Analogue Scale (VAS) consists of a $10 \mathrm{~cm}$ straight line without scale markers and with descriptors at each end. One VAS item on the degree of fatigue (Table 1) was included in the measurement tool.

The Multidimensional Fatigue Inventory (MFI) is a 20-item scale designed to measure multiple aspects of fatigue experienced during the last few days ${ }^{15}$. The MFI is based on the notion of fatigue being a multidimensional experience,

which can be expressed through five subscales of four items each. English and Dutch versions of the MFI have previously been validated in cancer patients undergoing radiotherapy ${ }^{15-17}$. All subscales of the MFI were included in the measurement tool.

The European Organization for Research and Treatment of Cancer Quality of Life Questionnaire (EORTC QLQ-C30), version 3.0, is a 30-item cancer-specific scale addressing various aspects of $\mathrm{HRQoL}^{18}$. It comprises of five functioning subscales (physical, role, emotional, cognitive and social functioning), three multi-item symptom scales (fatigue, pain and nausea/vomiting), two global health/quality of life subscales, and a number of single items addressing various symptoms and perceived financial impact. All subscales were included in the measurement tool.

The Maastricht Questionnaire (MQ) was developed at Maastricht University ${ }^{22}$ to measure vital exhaustion. The scale is one-dimensional and comprises of 23 items on abnormal fatigue, loss of energy, increased irritability, and feelings of demoralization. All items were included in the measurement tool.

The Rotterdam Symptom Checklist (RSCL) is a 39-item scale, which assesses symptoms during the preceding week ${ }^{19}$. The reliability and validity of the RSCL in cancer patients have been confirmed previously ${ }^{6,20,21}$. The RSCL assesses four domains: physical symptoms, psychological distress, activity level, and overall quality of life. 
Because of major overlap with items in other questionnaires included in the measurement tool, the subscale physical symptoms was excluded.

Table 1 Overview of used scales: subscales, examples of items and answer options

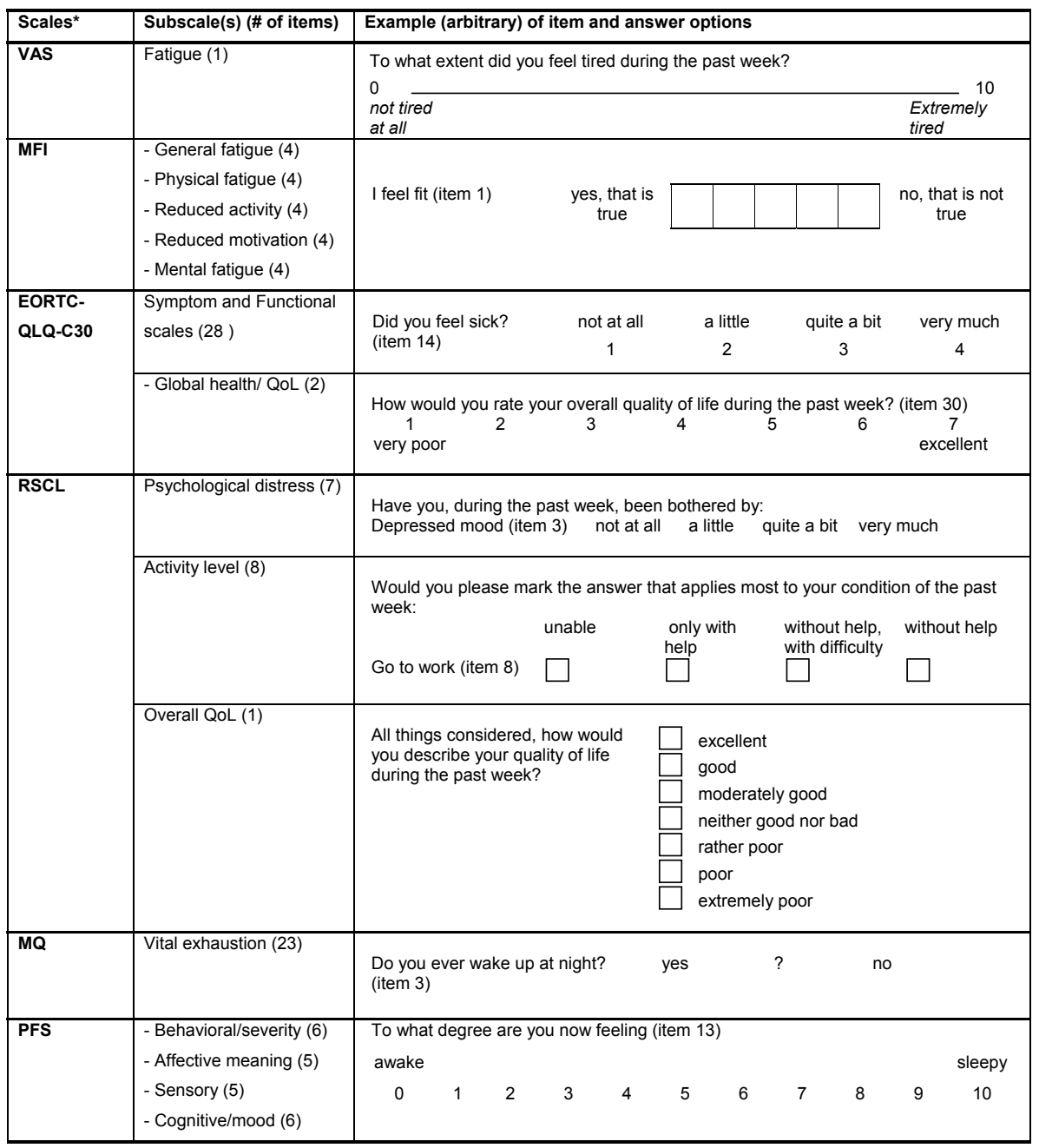

The Piper Fatigue Scale (PFS) ${ }^{23}$ is composed of 22 numerical items which assess fatigue as experienced at present. The PFS measures four dimensions of subjective fatigue: behavioural/severity, relating to the severity, distress, and degree of disruption in activity of daily living; affective meaning, relating to the emotional meaning attributed to fatigue; sensory, relating to the physical symptoms of fatigue; and cognitive/mood, relating to mental and mood states. All items were included in the measurement tool. For the present study, the PFS was translated into Dutch independently by three native speaking Dutch researchers. Thereafter, the translations of the three 
researchers were discussed in several consensus meetings that included all authors except AP and PL.

\section{Preferences and acceptability}

To evaluate the preferences and acceptability of the measurement tool, three approaches were followed. First, patients were asked to mark, during completion of the measurement tool, the items they found difficult to answer. Second, we analysed the numbers of missing values for each item, assuming that a high proportion of missing values partly reflects a low understanding or a low acceptability of that specific item or (sub-)scale. Third, patients were asked, after completion of the measurement tool, to fill out an evaluation form on which they could indicate which answer options they preferred the least and the most. Next to it, the number of patients that needed assistance (read questions aloud) in completing the measurement tool was registered.

\section{Outcome measures and statistical analysis}

Student t-test and Fisher's Exact test were used to compare differences in continuous and categorical variables, respectively, between tumour groups. Evaluation of the measurement tool was first done by calculating the proportion of missing values per item and per (sub-)scale:

$\frac{\text { Number of missing values }}{N_{\text {respondent }} \times \text { total number of items of scale }} \times 100$

Secondly, the proportion of items marked as difficult to answer per (sub-)scale was calculated:

$\frac{\text { Number of difficult items }}{N_{\text {respondent }} \times \text { total number of items of scale }} \times 100$

Thirdly, Pearson's correlations were used to evaluate the association between missing values and items rated as difficult. Finally, the proportion of patients that had indicated a specific answer option as the most and the least preferred was computed. The level of statistical significance was set at 0.05 (two-sided).

\section{RESULTS}

\section{Patient' characteristics}

Out of 70 patients invited for the study, 62 (89\%) completed the measurement tool. Eight patients, of whom seven were lung cancer patients, refused to cooperate be- 
cause they were too tired. The characteristics of the 62 patients who completed the study are presented in Table 2. Lung cancer patients, compared to breast cancer patients, were older $(p=0.014)$, had a lower Body Mass Index $(p=0.005)$ and a poorer WHO performance status $(p=<0.001)$. Of the 27 lung cancer patients, 23 had nonsmall-cell lung cancer (stage I: $n=4$, stage II: $n=5$, stage III: $n=10$, and not staged: $n=2$, according to the TNM staging classification system ${ }^{24}$ (results not tabulated), and six patients had small-cell lung cancer (limited disease). Of the 35 breast cancer patients, 23 patients had stage I, 9 patients stage II, 1 patient stage III, and 2 patients were not staged (TNM, not tabulated). Compared to patients with breast cancer, relatively more lung cancer patients had been pre-treated with chemotherapy $(p=0.021)$.

Table 2: Demographic and Clinical Characteristics of the Study Population $(\mathrm{N}=62)$.

\begin{tabular}{llll}
\hline & Lung cancer $(\mathbf{n}=\mathbf{2 7})$ & Breast cancer $(\mathbf{n}=\mathbf{3 5})$ & $\mathbf{P}^{*}$ \\
Age (years) & 67.8 & 60.2 & 0.014 \\
Mean & 11.0 & 12.4 & \\
SD & & & 0.005 \\
Body Mass Index (BMI) & 22.9 & 25.8 & \\
Mean & 3.0 & 3,9 & $<0.001$ \\
SD & 44 & 100 & \\
Sex (Female, \%) & & & \\
WHO Performance status (\%) & 22 & 94 & \\
0 & 48 & 6 & \\
1 & 11 & 0 & \\
2 & 19 & 0 & 0.021 \\
Unknown & & & \\
Pre-treatment (\%) & & 26 & \\
Chemotherapy & 56 & 100 & 0 \\
Surgery & 0 & 0 &
\end{tabular}

* Tests for mean age and BMI were performed using Student T-tests. Tests for WHO Performance status and Pre-treatment were performend using Fishers's Exact tests.

\section{Preference and acceptability}

In Table 3, the number of patients with a certain number of missing values per (sub-)scale, and the proportion of missing values, are presented per (sub-)scale. The (sub-)scales with the smallest proportion of missing values $(<1 \%)$ were the MFI, the EORTC QLQ-C30, the subscales psychological distress and overall QoL of the RSCL, and the MQ. A relatively large proportion of missing values was observed in the VAS (3.2\%), the RSCL subscale activity level (4.2\%) and the PFS (2.5\%). The high proportion for the PFS was attributed to the subscales behavioral/severity $(3.1 \%)$ and affective meaning $(6.6 \%)$ (not tabulated). Items with missing values in five 
or more patients were item 8 of the RSCL subscale activity level ( $n=20$, ability to go to work), item 9 of the $M Q(n=6$, sexual activities), and the PFS-items $4(n=7$, sexual activities), item 9 ( $n=5$, evaluation of fatigue on a 10-point scale with descriptors "protective-destructive") and item $10(n=5$, evaluation of fatigue on a 10-point scale with descriptors "positive-negative").

Table 3: Missing and difficult items per (sub-)scale.

\begin{tabular}{|c|c|c|c|}
\hline & Lung cancer ( $n=27$ ) & Breast cancer $(n=35)$ & $\mathbf{P}^{*}$ \\
\hline \multicolumn{4}{|l|}{ Age (years) } \\
\hline Mean & 67.8 & 60.2 & 0.014 \\
\hline SD & 11.0 & 12.4 & \\
\hline \multicolumn{4}{|l|}{ Body Mass Index (BMI) } \\
\hline Mean & 22.9 & 25.8 & 0.005 \\
\hline SD & 3.0 & 3,9 & \\
\hline Sex (Female, \%) & 44 & 100 & \\
\hline WHO Performance status (\%) & & & $<0.001$ \\
\hline 0 & 22 & 94 & \\
\hline 1 & 48 & 6 & \\
\hline 2 & 11 & 0 & \\
\hline Unknown & 19 & 0 & \\
\hline \multicolumn{4}{|l|}{ Pre-treatment (\%) } \\
\hline Chemotherapy & 56 & 26 & 0.021 \\
\hline Surgery & 0 & 100 & \\
\hline Radiotherapy & 0 & 0 & \\
\hline
\end{tabular}

Also presented in Table 3, is the proportion of items indicated by patients as 'difficult to answer'. (Sub-)scales with a relatively small proportion of items marked as difficult $(<1 \%)$ were the MFI, the EORTC QLQ-C30 subscales symptom and functioning, and the RSCL subscales. In contrast, patients' evaluation showed a relatively large proportion of difficult items for the subscale global health of the EORTC QLQ-C30 $(6.3 \%)$, and for the PFS $(3.4 \%)$. Also a relatively large proportion of the patients $(3.1 \%)$ evaluated the VAS as difficult.

The MFI and two subscales of the RSCL (activity level, distress) had hardly any items marked by patients as difficult. In contrast, a considerable number of items were marked as difficult to answer, for the EORTC QLQ-C30, the MQ and the PFS. Within the EORTC QLQ-C30, patients had most difficulties with the two items (both rated on a 7-point scale) of the subscale global health/Qol, which refer to the patients' opinion on their overall well-being and quality of life. For the $M Q$, items marked as difficult to answer were scattered across the scale, except for item 9 (referring to whether patients enjoy sexual activities nowadays as much as in the past), 
which was marked as difficult by seven patients. Of the PFS, items marked most frequently as difficult to answer were items $4(n=8)$ and $9(n=8)$. Item 4 refers to the interference of fatigue with sexual activity (10-point scale with descriptors "not at all very strong") and item 9 refers to the evaluation of fatigue (10-point scale with descriptors "protective - destructive").

Overall, a significant correlation $(r=0.27, p=0.004)$ was found between the proportion of missing values per item and the number of times this item was evaluated as difficult by patients (Table 4). However, this was not necessarily the case for all individual items. Separately from the total correlation, the correlation of the three items with the highest number of missing values and number of times these items were evaluated as difficult, were calculated.

\section{DISCUSSION}

To our knowledge, this is the first report on preference for and acceptability of selfassessment health related quality of life questionnaires among cancer patients prior to curative radiotherapy: The following tentative conclusions can be drawn: (1) patients appear to prefer answer options that have been described in words (e.g. $\mathrm{RSCL}$ ) in stead of only numbers (e.g. the RSCL) or have no label at all (e.g. the PFS), (2) patients appear to prefer questions with a narrow range of answer options (maximum of 4 or 5), and to dislike broad range answer formats (e.g. 10 points or VAS) . In addition, if the topic of certain items is either not applicable (for example the assessment of the ability to go to work when a patient already has retired) or too sensitive (e.g. sexual activities), this may lead to a relatively large number of missing answers.

Despite the unique approach in the present study, several limitations must be noted. First, since the study population is relatively small and the number of questionnaires is substantial, which may have affected the reliability of our results, our findings should be confirmed in subsequent larger studies. Second, since we included lung and breast cancer patients selected for curative radiotherapy, it could be argued that our conclusions may only apply to these specific patient groups. However, despite differences between lung and breast cancer patients with respect to age, BMI, WHO performance status, tumour stage and pre-treatment with chemotherapy, the overall results with regard to preference and acceptability were similar in both tumour groups, which would suggest that different patient groups feel similar about these HRQoL assessment tools. Third, the number of HRQoL assessment tools which are available to cancer patients is numerous. For reasons of subject burden, we could include only a relatively small selection of these in the present study. We chose a number of well-known and well-validated (sub-)scales, covering the most frequently reported symptoms of $\mathrm{HRQ}$ oL, and its sub-domain fatigue, in cancer patients. 
There may be several reasons why patients do not fill out a question in selfassessment HRQoL questionnaires. The topic of the question may not be applicable (anymore) to the patient, the answer option may be found difficult, the patient may find the subject of the question hard to empathize with, or he/she may consider it offensive or too sensitive.

Item 8 of the RSCL-activity level, was missed 20 times and was therefore responsible for the highest proportion of missing values $(4.2 \%)$ of this subscale within the measurement tool. An explanation for this result is that this item refers to the ability to go to work, whereas the majority of patients in the present study were either retired or had no paid job. Other examples of a high number of missing values due to the subject of the specific question were questions related to sexual activities (item 9 of the MQ and item 4 of the PFS). This subject may be too sensitive to answer for a considerable proportion of the present study population. Furthermore, the relatively large number of missing values of the PFS subscale affective meaning $(6.6 \%$, not tabulated) is remarkable. Items in this subscale refer to the extent to which fatigue is experienced by patients as agreeable vs. disagreeable, protective vs. destructive, positive vs. negative, and normal vs. abnormal. Apparently, these items are not offensive, but the way the questions are formulated or the concept covered by the four items of this subscale may very well be difficult to grasp for a considerable proportion of patients. In the present study, a pattern of the type of answer options with regard to the proportion of missing and difficult items was identified. The number of missing values in scales with a "broad range" of answer categories per item (range: $0.8 \%-3.1 \%$ ) was relatively high when compared to the number of missing values in scales with a "narrow range" of answer categories (range $0.0 \%-0.8 \%$ ). This pattern also applies to the proportion of difficult items per scale, with exception of the RSCL-overall QoL $(0.0 \%)$. All scales with a "broad range" of answer options appeared to have a considerably higher proportion of items indicated as difficult to answer (range: $3.1 \%-6.3 \%$ ), when compared to scales with a "narrow range" of answer options per item (range: $0.0 \%$ $1.0 \%$ ). A plausible explanation for the observation that the subscale overall QoL of the RSCL is an exception (one item, never missed), is that all answer options of this 7-point sub-scale are described in words in the scale. This would suggest that, when answer options are described in words, the number of answer categories may be of lesser importance. However, it must be emphasized that a considerable part of the patients indicated that the kind of answer option made no difference to them.

The total time needed to fill out the measurement tool did not differ between the tumour groups. However, whereas none of the breast cancer patients needed assistance in completing the measurement tool, five lung cancer patients, all with performance status WHO-2, needed assistance to complete the measurement tool. Although this finding is based on a small number of patients, the possibility must be taken into account that a relatively large proportion of patients with poor performance status $(\geq 2)$ may need assistance in order to complete HRQoL assessment. . 
In conclusion, the results of the present study indicate that, when evaluating the effect of clinical and psychosocial interventions in cancer patients, respondents may prefer HRQoL measurement tools with answer categories that have either descriptors in words, or a relatively narrow range of answer options per question. Furthermore, to obtain valid data from HRQoL scales, the characteristics of the study population must be taken into account, in order to estimate if questions are either not applicable, too sensitive, or offensive.

These findings may help in designing and choosing patient-friendly HRQoL scales, which will lead to a reduction in non-response or missing items in HRQoL assessment. 


\section{REFERENCES}

1. Bottomley A, Efficace F, Thomas R, Vanvoorden V, Ahmedzai SH. Health-related quality of life in nonsmall-cell lung cancer: methodologic issues in randomized controlled trials. $\mathrm{J}$ Clin Oncol 2003;21(15):2982-92.

2. Langendijk JA, Aaronson NK, de Jong JM, ten Velde GP, Muller MJ, Lamers RJ, et al. Prospective study on quality of life before and after radical radiotherapy in non-small-cell lung cancer. J Clin Oncol 2001;19(8):2123-33.

2. Velikova G, Booth L, Smith AB, Brown PM, Lynch P, Brown JM, et al. Measuring quality of life in routine oncology practice improves communication and patient well-being: a randomized controlled trial. J Clin Oncol 2004;22(4):714-24.

3. Aaronson NK, Ahmedzai S, Bergman B, Bullinger M, Cull A, Duez NJ, et al. The European Organization for Research and Treatment of Cancer QLQ-C30: a quality-of-life instrument for use in international clinical trials in oncology. J Natl Cancer Inst 1993;85(5):365-76.

4. Cella DF, Bonomi AE, Lloyd SR, Tulsky DS, Kaplan E, Bonomi P. Reliability and validity of the Functional Assessment of Cancer Therapy-Lung (FACT-L) quality of life instrument. Lung Cancer 1995;12(3):199-220.

5. Ganz PA, Schag CA, Lee JJ, Sim MS. The CARES: a generic measure of health-related quality of life for patients with cancer. Qual Life Res 1992;1(1):19-29.

6. de Haes JC, van Knippenberg FC, Neijt JP. Measuring psychological and physical distress in cancer patients: structure and application of the Rotterdam Symptom Checklist. Br J Cancer 1990;62(6):10348.

7. Schipper H, Clinch J, McMurray A, Levitt M. Measuring the quality of life of cancer patients: the Functional Living Index-Cancer: development and validation. Journal of Clinical Oncology 1984;2:472-483.

8. Rodary C, Pezet-Langevin V, Garcia-Acosta S, Lesimple T, Lortholary A, Kaminsky MC, et al. Patient preference for either the EORTC QLQ-C30 or the FACIT Quality Of Life (QOL) measures: a study performed in patients suffering from carcinoma of an unknown primary site (CUP). Eur $J$ Cancer 2004;40(4):521-8.

9. WHO. The WHO handbook for reporting results of cancer treatment. Geneva: World Health Organisation; 1979.

10. Smets EM, Garssen B, Schuster-Uitterhoeve AL, de Haes JC. Fatigue in cancer patients. Br J Cancer 1993;68(2):220-4.

11. Richardson A. Fatigue in cancer patients: a review of the literature. Eur $\mathrm{J}$ Cancer Care (Engl) 1995;4(1):20-32.

12. Bower JE, Ganz PA, Desmond KA, Rowland JH, Meyerowitz BE, Belin TR. Fatigue in breast cancer survivors: occurrence, correlates, and impact on quality of life. J Clin Oncol 2000;18(4):743-53.

13. Cheung YB, Wong LC, Tay MH, Toh CK, Koo WH, Epstein R, et al. Order effects in the assessment of quality of life in cancer patients. Qual Life Res 2004;13(7):1217-23.

14. Childs AL. Effect of order of administration of health-related quality of life interview instruments on responses. Qual Life Res 2005;14(2):493-500.

15. Smets EM, Garssen B, Bonke B, De Haes JC. The Multidimensional Fatigue Inventory (MFI) psychometric qualities of an instrument to assess fatigue. J Psychosom Res 1995;39(3):315-25.

16. Meek PM, Nail LM, Barsevick A, Schwartz AL, Stephen S, Whitmer K, et al. Psychometric testing of fatigue instruments for use with cancer patients. Nurs Res 2000;49(4):181-90.

17. Smets EM, Garssen B, Cull A, de Haes JC. Application of the multidimensional fatigue inventory (MFI20) in cancer patients receiving radiotherapy. Br J Cancer 1996;73(2):241-5.

18. McLachlan S-A, Devins GM, Godwin PJ. Validation of the European Organisation for Research and Treatment of Cancer quality of life questionaire (QLQ-C30) as a measure of psychosocial function in breast cancer patients. European journal of cancer 1998;34(4):510-517. 
19. de Haes JCM, Olschewski M, Fayers P, Visser MRM, Cull A, Hopwood P, et al. Measuring the quality of life of cancer patients with the Rotterdam Symptom Checklist (RSCL): a Manual.; 1996.

20. Hopwood P, Howell A, Maguire P. Screening for psychiatric morbidity in patients with advanced breast cancer: validation of two self-report questionnaires. Br J Cancer 1991;64(2):353-6.

21. Ravaioli A, Buda P, Fava C, Paci E, Tononi A, Riva N, et al. Assessment of the RSCL quality of life instrument during chemotherapy in an Italian setting. Qual Life Res 1996;5(5):491-5.

22. Appels A, Hoppener P, Mulder P. A questionnaire to assess premonitory symptoms of myocardial infarction. Int J Cardiol 1987;17(1):15-24.

23. Piper BF, Dibble SL, Dodd MJ, Weiss MC, Slaughter RE, Paul SM. The revised Piper Fatigue Scale: psychometric evaluation in women with breast cancer. Oncol Nurs Forum 1998;25(4):677-84.

24. Cancer IUA. TNM classification of malignant tumors. 5 ed. New York: Wiley-Liss; 1997. 



\section{CHAPTER}

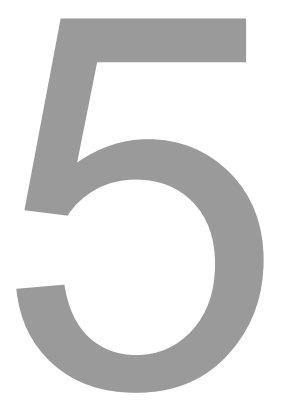

\section{Impact of fatigue on overall quality of life in lung and breast cancer patients selected for high-dose radiotherapy}

Annals of Oncology (2007), 18: 940-944

Pieter Dagnelie

Madelon Pijls-Johannesma

Philippe Lambin

Sandra Beijer

Dirk De Ruysscher

Ruud Kempen 


\begin{abstract}
Background: Although studies show that cancer patients consider fatigue as an important problem, few if any studies have quantified the impact of fatigue on overall quality of life (QoL) in cancer patients. In the present study, we evaluated the relative impact of somatic, psychological, social, and fatigue domains on overall QoL in cancer patients preceding radiotherapy.
\end{abstract}

Patients and methods: Sixty-four patients with lung or breast cancer selected for high-dose radiotherapy on the primary tumour completed the EORTC QLQ-C30 questionnaire. Multivariate models were fitted to define the impact of subscales within four QoL domains (somatic, psychological, social, fatigue) on overall QoL.

Results: Of all QLQ-C30 subscales, fatigue showed by far the strongest univariate correlation with overall QoL $(r=-0.76, p<0.001)$; correlations for functioning subscales $(r=0.44-0.55)$ and symptom subscales $(r=-0.31--0.45)$ were considerably lower. In multivariate analyses, fatigue paid the greatest contribution to overall QoL (standardized regression coefficient (SRC): $-0.57, \mathrm{p}<0.001$ ), with nausea/vomiting being the only other subscale that independently contributed to QoL (SRC: -0.22 , $\mathrm{p}<0.05)$.

Conclusion: Our results indicate that, of all QoL domains/subscales, fatigue is by far the predominant contributor to patient-perceived overall QoL in both lung and breast cancer patients preceding high-dose radiotherapy. 


\section{INTRODUCTION}

Over the last decade, Quality of Life (QoL) measures have been routinely incorporated in oncological clinical trials; also, the use of QoL measures in clinical settings is strongly encouraged because their value in cancer patient management has now been established ${ }^{1}$. QoL is generally considered as a multidimensional construct, which includes several key dimensions ${ }^{2}$.

Fatigue (defined as a general feeling of debilitating tiredness or loss of energy) is one of the most common quality of life related symptoms in cancer today ${ }^{3-8}$. The occurrence of fatigue varies across tumor sites ${ }^{6,9,10}$ and treatment stage ${ }^{6,9,11-14}$. Lung cancer patients undergoing radiotherapy had the highest levels of fatigue relative to patients of other tumour types ${ }^{6}$. For patients and oncologists, improving the quality of life of cancer patients requires a heightened awareness of fatigue, a better understanding of its impact, and improved communication and familiarity with interventions that can reduce its debilitating effects ${ }^{15}$. However, despite previous surveys on patients' subjective and conscious valuation of the impact of fatigue on daily life, few if any studies so far have made an attempt to quantify the impact of fatigue, relatively to other QoL domains, on overall QoL in cancer patients prior to high-dose radiotherapy. In the present paper, we will address this issue using the three-level QoL structure as proposed by Spilker et $\mathrm{al}^{16}$. According to these authors, the top level of QoL is referred to in clinical trial literature as "global QoL assessment" and can be interpreted as an overall impression of "an individual's overall satisfaction with life, and one's general sense of personal well-being". This level corresponds to the WHO definition of QoL as "a state of complete physical, mental and social well-being and not merely the absence of diseases and infirmity". The second or middle level represents several broad dimensions or domains of QoL. The most widely used domains of QoL are physical/somatic, psychological and social functioning; in some studies, economic status, cognitive functioning, personal productivity, intimacy and spiritual domains are added ${ }^{17}$. Finally, the bottom level contains the components of each domain that are specifically assessed as items in QoL questionnaires. For example, functional limitations or activities of daily living are part of the physical domain of QoL; measures of anxiety or depression are specific aspects of the domain of psychological functioning, whereas participation in social activities can give an impression of the domain of social functioning.

Spilker's model ${ }^{16}$ assumes that QoL variables at a lower level determine QoL variables at a higher level. This relation between lower and higher QoL levels has great practical relevance, because it gives insight in the consequences of an illness for patients in terms of valuation of life. An overall judgement of QoL requires patients to evaluate several life domains, and to combine the weightings of these domains into a "generic value judgement" of life. 
So far, only few studies have compared disorders with respect to $\mathrm{QoL}^{17-21}$. Arnold and colleagues ${ }^{17}$, for example, recently studied the contribution of physical, social and psychological functioning to patients' valuation of overall QoL in a cross-sectional study in healthy subjects and patients with chronic disorders. However, no cancer patients were included in that study. Linear regression analyses showed that the domain of psychological functioning contributed to overall QoL for all disorders, whereas physical and social functioning contributed to overall QoL for some disorders only.

The contribution of different QoL dimensions to overall QoL in cancer patients could differ from chronic diseases for several reasons. First, in contrast with many chronic diseases, cancer is perceived as a more acutely or sub-acutely life-threatening disease, and second, symptoms such as fatigue, anorexia and pain play a predominant and specific role in the quality of life of cancer patients ${ }^{22-24}$, and might therefore be valuated differently by cancer patients relative to patients with other acute and chronic diseases.

Therefore, the aim of the present study was to quantify the impact of fatigue, relative to other QoL domains, on overall QoL in cancer patients preceding radiotherapy.

\section{PATIENTS AND METHODS}

\section{Study population and design}

The present study used a cross-sectional design to assess fatigue, several QoL domains and overall QoL in a population of lung and breast cancer patients selected for curative high-dose radiotherapy ( $\geq 50 \mathrm{~Gy}$ ) on the primary tumour. Seventy patients older than 18 years of age and with World Health Organization (WHO) performance status 0-2 were invited to participate ${ }^{25}$. Both breast cancer patients and non-surgery lung cancer patients were included. Prior to radiotherapy, patients were asked to complete a measurement tool including a demographic profile and the well-known European Organization for Research and Treatment of Cancer Quality of Life Questionnaire (EORTC QLQ-C30). After permission, both the patient and their radiation oncologist signed for informed consent. The study was approved by the ethics committee of MAASTRO clinic, Maastricht, The Netherlands.

\section{MEASUREMENT INSTRUMENTS}

Background variables included age, gender, body mass index, WHO performance status and prior treatment. The EORTC Quality of Life Questionnaire (QLQ-C30, version 3.0) is a 30-item cancer-specific core questionnaire that addresses various domains of $\mathrm{QoL}^{26}$. It contains five function subscales (physical functioning, role func- 
tioning, emotional functioning, cognitive functioning and social functioning), three symptom subscales (fatigue, pain and nausea/vomiting), two single items assessing global health and "overall" quality of life, and a number of single items addressing various symptoms and perceived financial impact.

\section{Statistical analysis}

Pearson's correlation analyses were calculated to study the relation between QoL subscales and overall QoL. As dependent variable "overall QoL", we used, as mentioned above, item 30 of the EORTC QLQ-C30 ("How would you rate your overall quality of life during the past week?"). To assess the contribution of different subscales to overall QoL, QLQ-C30 subscales were clustered into three major QoL domains: 1. Somatic, comprising the functional subscale physical functioning and the symptom subscales: nausea/vomiting, pain, dyspnoea, appetite loss, constipation and diarrhoea; 2. Psychological, including the functional subscales: emotional and cognitive functioning, as well as the symptom subscales: insomnia and financial difficulties; and 3. Social, including the social and role functioning subscales. In order to assess the impact of fatigue relative to other QoL domains, the Fatigue symptom subscale was included in all analyses as a separate variable.

Linear regression models were fitted to investigate the impact of functioning and symptom and subscales within four domains, i.e. somatic, psychological, social, and fatigue, on overall QoL. As independent variables, we included only "major" QLQ-C30 subscales, defined as subscales which individually explained at least $20 \%$ of the variance of overall QoL in the univariate analyses (i.e. subscales showing correlations with overall QoL of $r \geq 0.45$ or $\leq-0.45$ ). The dependent variable in these regression analyses was overall QoL; age, gender and tumour type were included as potential confounders. First, one model was fitted to assess the impact of the "major" subscales within the somatic, psychological, social and fatigue domains on overall QoL. Then, to compare the impact of fatigue, relative to subscales from the three other domains, on overall QoL, two additional models were fitted: one model which included as covariates the "major" subscales of the somatic, psychological, social domain as covariates, but not fatigue; and one model which included fatigue in addition to the three other domains. An $F$ test ( $F$ change) was used to compare the variance $\left(R^{2}\right)$ in overall QoL explained by different models.

Presented $R^{2}$ values in this paper are adjusted $R^{2}$ values throughout. Collinearity tests showed that absence of multicollinearity between any of the determinants included in the regression models. P-values $<0.05$ (two-tailed) were considered statistically significant. 


\section{RESULTS}

\section{Patient characteristics}

Six patients out of the 70 included patients refused to cooperate because of being too tired; the remaining 64 patients $(91 \%)$ completed the EORTC QLQ-C30 questionnaire. Patient characteristics are presented in Table 1.

Mean age of the study population was 64 years, and the large majority of patients had WHO performance status 0 or 1 . Half of the lung cancer and a quarter of the breast cancer patients had been pretreated with chemotherapy. EORTC QLQ-C30 scores showed considerable impairment in QoL, especially in lung cancer patients, for the subscale "global health status / QoL" and its components "global health" (QLQ-C30 item 29) and "overall QoL" (QLQ-C30 item 30), as well as for the subscales physical, role and emotional functioning, and for the symptom subscales fatigue, dyspnoea, pain, insomnia and appetite loss. Lung cancer patients were significantly more tired than breast cancer patients $(p<0.01)$; also, prior treatment with chemotherapy was also associated with higher fatigue levels $(p<0.05)$.

\section{Contribution of different QoL subscales and domains to overall QoL}

Univariate correlations between functional and symptom subscales of the QLQ-C30 and overall QoL are shown in Table 2. Of all subscales, fatigue showed by far the strongest correlation with overall QoL $(r=-0.76, p<0.001)$. Other subscales with statistically significant correlations with overall QoL were: all functional subscales $(r=$ $0.44-0.55$ ), and (in decreasing order) the symptom subscales loss of appetite, nausea/vomiting, insomnia, dyspnoea, pain and constipation $(r=-0.45--0.31)$. The correlation between fatigue and overall QoL was independent of tumour type (lung cancer: $r=-0.74$; breast cancer: $r=-0.74$; both $p<0.001$ ). Correlations between other subscales and overall QoL were also similar in lung and breast cancer patients (data not shown), except for dyspnoea (lung cancer: $r=-0.55, p<0.01$; breast cancer: $0.098, N S$ ) and financial difficulties (lung cancer: $r=-0.20$, NS; breast cancer: -0.38 , $\mathrm{p}<0.05)$.

In Table 3, the contributions of subscales within different QoL domains (somatic, psychological, social, fatigue) to overall QoL are shown. In these analyses, only subscales explaining $\geq 20 \%$ of the variance of QoL in both tumour types combined (corresponding with univariate correlations $\geq 0.45$ or $\leq-0.45$ in Table 2) were included.

After adjustment for age, gender and tumour type, fatigue alone explained $56 \%$ of the variance in overall QoL $(p<0.001)$. In contrast, the combined subscales within the physical, social and psychological domain explained only $43 \%, 32 \%$ and $28 \%$, respectively, of the variance in overall QoL $(p<0.001)$. 
Table 1: Characteristics of the study population $(\mathrm{N}=64)$ by tumour type.

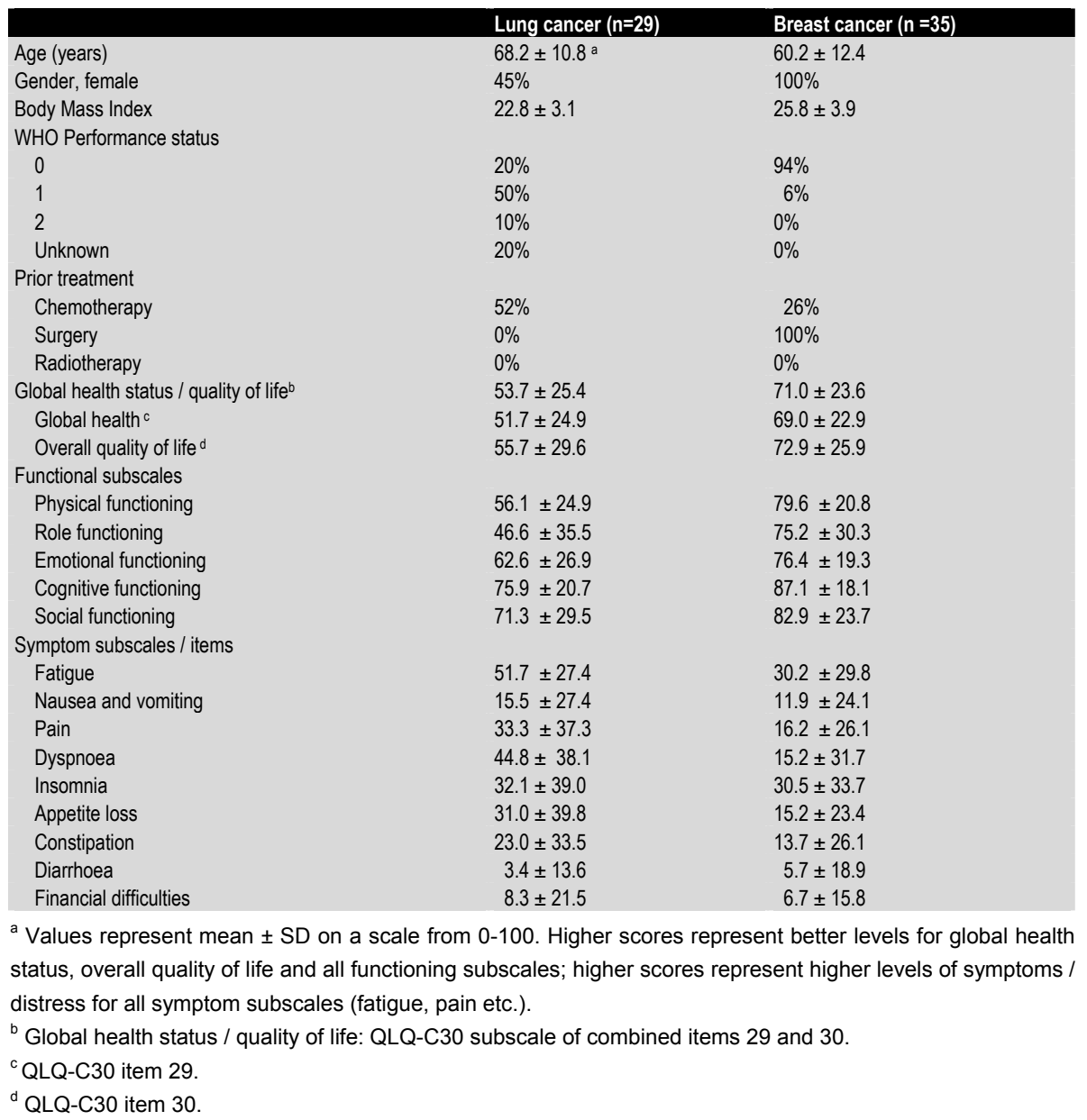

Of all individual subscales, fatigue paid by far the highest contribution to overall QoL (standardized regression coefficient: $-0.73, p<0.001$ ). The second most important determinant of overall QoL was the subscale physical functioning within the somatic domain (standardized regression coefficient: $0.42, p<0.01$ ), followed by the symptom subscale nausea/vomiting within the same domain (standardized regression coefficient: $-0.35, p<0.01)$. Within the psychological domain, emotional functioning contributed less but still significantly to overall QoL (standardized regression coefficient: $0.45, p<0.001$ ). Within the social domain, only social functioning contributed significantly to overall QoL (standardized regression coefficient: 0.36, $\mathrm{p}<0.01$ ). 
Table 2: Pearson's correlations between different EORTC-QLQ-C30 subscales and overall quality of life.

\begin{tabular}{llr}
\hline & $\begin{array}{l}\text { Correlation } \\
(\mathbf{n}=64)\end{array}$ & p-value \\
\hline Physical functioning & 0.550 & $<0.001$ \\
Role functioning & 0.515 & $<0.001$ \\
Emotional functioning & 0.515 & $<0.001$ \\
Cognitive functioning & 0.441 & $<0.001$ \\
Social functioning & 0.507 & $<0.001$ \\
Fatigue & -0.759 & $<0.001$ \\
Nausea and vomiting & -0.451 & $<0.001$ \\
Pain & -0.361 & 0.004 \\
Dyspnoea & -0.416 & 0.001 \\
Insomnia & -0.418 & 0.001 \\
Appetite loss & -0.452 & $<0.001$ \\
Constipation & -0.314 & 0.012 \\
Diarrhoea & -0.077 & 0.548 \\
Financial difficulties & -0.280 & 0.026
\end{tabular}

Table 3 also gives two overall models: one showing the independent contributions of relevant subscales within the somatic, psychological and social domains, and one model with the same subscales plus fatigue. It is seen that the overall model without fatigue explained $48 \%$ of the variance of QoL, i.e. less than the fatigue subscale alone $(56 \%)$. Comparison of these two overall models, i.e. without and with fatigue, showed that adding fatigue to the total model significantly increased the explained variance of overall QoL from $48 \%$ to $57 \%(p<0.001)$. In contrast, the explained variance of the total model including fatigue $(57 \%)$ was not significantly different $(p=0.39)$ from the model with fatigue alone (48\%).

In the total model including fatigue, the fatigue subscale paid by far the highest individual contribution to overall QoL (standardized regression coefficient: -0.57 , $p=0.002)$. In this model, none of the other subscales contributed significantly to overall QoL.

Table 3: Independent contribution of different EORTC QLQ-C30 subscales to overall QoL, adjusted for age, gender, tumor stage and tumor type $(\mathrm{N}=64)$.

\begin{tabular}{lll} 
Subscale & $\begin{array}{l}\text { Model1: total model without fatique } \\
\left(\mathbf{R}^{2}=\mathbf{0 . 4 0 0 ^ { * * } )}\right.\end{array}$ & $\begin{array}{l}\text { Model 2: total model including fatigue } \\
\left(\mathbf{R}^{2}=\mathbf{0 . 5 0 2}\right.\end{array}$ \\
\hline Physical functioning & $0.371^{*}$ & 0.178 \\
Role functioning & -0.029 & -0.132 \\
Emotional functioning & $0.307^{*}$ & 0.194 \\
Social functioning & 0.104 & -0.016 \\
Nausea and vomiting & $-0.303^{*}$ & -0.207 \\
Appetite loss & -0.065 & 0.063 \\
Fatigue & ----- & $-0.574^{* *}$
\end{tabular}

Estimates show standardized regression coefficients for different QoL domains and subscales, based on linear regression models with overall $Q$ oL as the dependent variable. Values of $R^{2}$ for each domain (somatic, psychological, social, fatigue, and total without / with fatigue) show the overall adjusted $R^{2}$ of the concerned regression model.

* $p<0.05$.,

** $p<0.01$,

*** $p<0.001$ 


\section{DISCUSSION}

The aim of the present study was to investigate the impact of fatigue, relative to other QoL domains, on overall QoL in cancer patients preceding radiotherapy. Results showed that fatigue was by far the predominant determinant of overall QoL, showing an univariate correlation with overall QoL of -0.76 . This correlation was independent of tumour type, and multivariate analyses showed that the relation between fatigue and overall QoL remained unchanged after adjustment for tumour type, age and gender. In fact, fatigue alone explained a greater proportion of variance in overall QoL than the combined functional and symptoms subscales of all other domains (somatic, psychological, social). This finding suggests that, in our study population, the impact of fatigue on overall QoL largely overruled the impact of any other functional and symptom subscales.

So far, little research has been conducted to quantitatively assess the empirical relations between physical, psychological and social domains and overall QoL in cancer patients. The contribution of specific domains to overall QoL may differ between diseases since different diseases may have a differential effect on the functioning of patients in different QoL domains. In a previous study in patients with different chronic disorders, but not cancer ${ }^{17}$, the correlations between different functional QoL domains and overall QoL were considerably lower (mostly $r \leq 0.45$ ) than in our cancer population, and the variance in overall QoL explained by combined physical, psychological and social domains was $<30 \%$ for all conditions except lung disorders $(33 \%)$ and migraine $(44 \%)$. The psychological functioning domain was the predominant determinant in all chronic conditions, whereas physical functioning contributed to overall QoL for lung disorders only ${ }^{17}$. Fatigue was not evaluated as a separate QoL domain by these authors.

Fatigue is a symptom frequently encountered in cancer patients, due to either illness or treatment. Almost every patient suffers from fatigue during cancer treatment such as radiotherapy and/or chemotherapy, and in the scientific literature, reported prevalence rates of fatigue are up to $99 \%{ }^{6,14,27,28}$. Research shows that chronic fatigue is associated with problems and limitations in different areas of life. Not only does severe fatigue lead to physical restrictions, but also to serious impairment in quality of life, social activities and the ability to go to work ${ }^{6,14,29,30}$. The present study, in which we evaluated the relation between fatigue scores of patients and their overall QoL scores on the EORTC QLQ-C30 questionnaire, corroborates the dramatic impact of fatigue on overall QoL.

In our study, we purposely did not use the subscale "global health status / QoL" of the QLQ-C30 as the dependent variable, because this subscale is the sum of two conceptually different items, which are related to global health (item 29) and overall quality of life (item 30), respectively. However, results remained quite similar when we tentatively repeated all analyses using the "global health status / QoL" subscale 
(sum of items 29 and 30 of the QLQ-C30) as the dependent variable (data not shown), giving further support to the importance of fatigue in patients' valuation of global health and QoL.

Strengths of the present study are the relatively homogeneous study population with only two tumour types at a well-defined treatment stage, i.e. prior to high-dose radiotherapy, as well as the high level of participation of subjects. We purposely included patients of two well-defined tumour types with considerable anticipated variation in physical and psychological distress. For instance, lung cancer is well-known as a tumour type with high frequency of weight loss and impaired physical fitness, whereas in breast cancer patients, changes in role-related and cognitive aspects of QoL would appear to play a more predominant role. Despite these differences, the contribution of fatigue to overall QoL was similar in lung and breast cancer patients, as shown by identical correlations between fatigue and overall QoL in both tumour types separately. Therefore, even though the relatively small number of subjects is a limitation of our study, is seems quite unlikely that a larger number of subjects would have changed its results. Although the present study was not aimed at investigating the aetiology of fatigue, tentative analyses indicated that the high level of fatigue in our patient population was unrelated to BMI, WHO score or hemoglobin levels (data not shown).

In conclusion, our results indicate that, in lung and breast cancer patients preceding radiotherapy, fatigue is by far the predominant contributor to patient-perceived overall QoL. Our results support the notion that fatigue in cancer patients is a major problem, which deserves yet more attention from health professionals, and that QoL in cancer patients might be improved markedly by interventions that effectively reduce fatigue. Further research is needed to reproduce these results in patients of different tumour types and at different stages of disease and therapy, ranging from diagnosis to the palliative treatment stage. 


\section{REFERENCES}

1. Soni MK, Cella D. Quality of life and symptom measures in oncology: an overview. Am J Manag Care. 2002;8(18 Suppl):S560-73.

2. Velikova G, Stark D, Selby P. Quality of life instruments in oncology. Eur J Cancer 1999;35(11):157180.

3. Curt GA. Fatigue in cancer. Bmj 2001;322(7302):1560.

4. Morrow GR, Shelke AR, Roscoe JA, Hickok JT, Mustian K. Management of cancer-related fatigue. Cancer Invest 2005;23(3):229-39.

5. Donovan KA, Jacobsen PB, Andrykowski MA, Winters EM, Balducci L, Malik U, et al. Course of fatigue in women receiving chemotherapy and/or radiotherapy for early stage breast cancer. J Pain Symptom Manage 2004;28(4):373-80.

6. Smets EM, Visser MR, Willems-Groot AF, Garssen B, Oldenburger F, van Tienhoven G, et al. Fatigue and radiotherapy: (A) experience in patients undergoing treatment. $\mathrm{Br} \mathrm{J}$ Cancer 1998;78(7):899-906.

7. Richardson A. Fatigue in cancer patients: a review of the literature. Eur $\mathrm{J}$ Cancer Care (Engl) 1995;4(1):20-32.

8. Winningham ML, Nail LM, Burke MB, Brophy L, Cimprich B, Jones LS, et al. Fatigue and the cancer experience: the state of the knowledge. Oncol Nurs Forum 1994;21(1):23-36.

9. Monga U, Kerrigan AJ, Thornby J, Monga TN. Prospective study of fatigue in localized prostate cancer patients undergoing radiotherapy. Radiat Oncol Investig 1999;7(3):178-85.

10. King KB, Nail LM, Kreamer K, StrohI RA, Johnson JE. Patients' descriptions of the experience of receiving radiation therapy. Oncol Nurs Forum 1985;12(4):55-61.

11. Borthwick D, Knowles G, McNamara S, Dea RO, Stroner P. Assessing fatigue and self-care strategies in patients receiving radiotherapy for non-small cell lung cancer. Eur J Oncol Nurs 2003;7(4):231-41.

12. Faithfull S. Fatigue in patients receiving radiotherapy. Prof Nurse 1998;13(7):459-61.

13. Visser MR, Smets EM. Fatigue, depression and quality of life in cancer patients: how are they related? Support Care Cancer 1998;6(2):101-8.

14. Irvine D, Vincent L, Graydon JE, Bubela N, Thompson L. The prevalence and correlates of fatigue in patients receiving treatment with chemotherapy and radiotherapy. A comparison with the fatigue experienced by healthy individuals. Cancer Nurs 1994;17(5):367-78.

15. Vogelzang NJ, Breitbart W, Cella D, Curt GA, Groopman JE, Horning SJ, et al. Patient, caregiver, and oncologist perceptions of cancer-related fatigue: results of a tripart assessment survey. The Fatigue Coalition. Semin Hematol. 1997;34(3 Suppl 2):4-12.

16. Spilker B. Introduction to the field of Quality of Life trials. In: Spliker B, editor. Qulity of life Assessment in Clinical Trials. New York: Raven Press; 1990. p. 3-10.

17. Arnold R, Ranchor AV, Sanderman R, Kempen GI, Ormel J, Suurmeijer TP. The relative contribution of domains of quality of life to overall quality of life for different chronic diseases. Qual Life Res 2004;13(5):883-96.

18. Ross SD, Fahrbach K, Frame D, Scheye R, Connelly JE, Glaspy J. The effect of anemia treatment on selected health-related quality-of-life domains: a systematic review. Clin Ther 2003;25(6):1786-805.

19. Rustoen T, Wahl A, Burchardt C. Changes in the importance of quality of life domains after cancer diagnosis. Scand J Caring Sci 2000;14(4):224-31.

20. Sprangers MA, de Regt EB, Andries F, van Agt HM, Bijl RV, de Boer JB, et al. Which chronic conditions are associated with better or poorer quality of life? J Clin Epidemiol 2000;53(9):895-907.

21. Stewart AL, Greenfield S, Hays RD, Wells K, Rogers WH, Berry SD, et al. Functional status and wellbeing of patients with chronic conditions. Results from the Medical Outcomes Study. Jama 1989;262(7):907-13.

22. Langendijk JA, ten Velde GP, Aaronson NK, de Jong JM, Muller MJ, Wouters EF. Quality of life after palliative radiotherapy in non-small cell lung cancer: a prospective study. Int J Radiat Oncol Biol Phys 2000;47(1):149-55. 


\section{2 | Part 2: QoL- Chapter 5}

23. Bower JE, Ganz PA, Desmond KA, Rowland JH, Meyerowitz BE, Belin TR. Fatigue in breast cancer survivors: occurrence, correlates, and impact on quality of life. J Clin Oncol 2000;18(4):743-53.

24. Smets EM, Visser MR, Willems-Groot AF, Garssen B, Schuster-Uitterhoeve AL, de Haes JC. Fatigue and radiotherapy: (B) experience in patients 9 months following treatment. $\mathrm{Br} J$ Cancer 1998;78(7):907-12.

25. Pijls-Johannesma MC, Pijpe A, Kempen GI, Lambin P, Dagnelie PC. Health related quality of life assessment instruments: A prospective study on preference and acceptability among cancer patients referred for radiotherapy. Eur J Cancer 2005;41(15):2250-6.

26. McLachlan S-A, Devins GM, Godwin PJ. Validation of the European Organisation for Research and Treatment of Cancer quality of life questionaire (QLQ-C30) as a measure of psychosocial function in breast cancer patients. European journal of cancer 1998;34(4):510-517.

27. Smets EM, Garssen B, Schuster-Uitterhoeve AL, de Haes JC. Fatigue in cancer patients. Br J Cancer 1993;68(2):220-4.

28. Servaes $P$, Verhagen $C$, Bleijenberg G. Fatigue in cancer patients during and after treatment: prevalence, correlates and interventions. Eur J Cancer. 2002;38(1):27-43.

29. Hurny C, Bernhard J, Joss R, Schatzmann E, Cavalli F, Brunner K, et al. "Fatigue and malaise" as a quality-of-life indicator in small-cell lung cancer patients. The Swiss Group for Clinical Cancer Research (SAKK). Support Care Cancer 1993;1(6):316-20.

30. Aaronson NK, Ahmedzai S, Bergman B, Bullinger M, Cull A, Duez NJ, et al. The European Organization for Research and Treatment of Cancer QLQ-C30: a quality-of-life instrument for use in international clinical trials in oncology. J Natl Cancer Inst 1993;85(5):365-76. 


\section{CHAPTER}

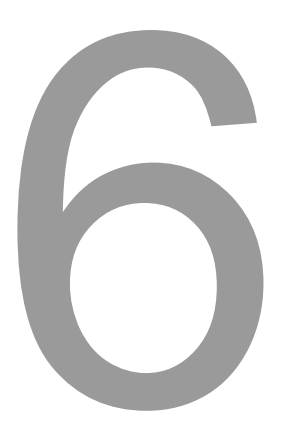

High dose radiotherapy or concurrent chemo-radiation in lung cancer patients only induces a temporary, reversible decline in QoL

Submitted

Madelon Pijls-Johannesma

Ruud Houben

Liesbeth Boersma Janneke Grutters

Katarina Seghers

Philippe Lambin

Dirk De Ruysscher 


\section{ABSTRACT}

Purpose: Aggressive radiotherapy or concurrent chemo-radiation therapy for lung cancer leads to a high incidence of severe, mostly esophageal, toxicity. The purpose of this study was to investigate the evolution of quality of life (QoL) in patients with lung cancer, selected for curative radiotherapy (RT) or chemo-RT.

Procedures: 75 lung cancer patients completed a longitudinal QoL measurement (from baseline till 18 months after RT), using the EORTC QLQ-C30 with the lung cancer module, EORTC QLQ-LC13. Esophageal toxicity was scored according to EORTC/RTOG criteria. Linear mixed regression models were fitted to longitudinally investigate the impact of different factors on overall QoL.

Results: Overall QoL decreased shortly after the end of RT (4 points, $p=0.19$ ), but increased back to baseline within 3 months. Mean scores of role functioning $(p=0.018)$, cognitive functioning $(p=0.002)$, dyspnoea (EORTC QLQ-LC13; $p=0.043$ ), dysphagia $(p=0.005)$ and hoarseness $(p=0.029)$, showed a significant worsening over time. Emotional functioning $(p=0.033)$ improved significantly over time. The remaining scores did not seem to change much over time.

Severe esophagitis ( $\geq$ grade 2 ) was reported in only $12 \%$ of the patients. Next to maximal esophageal toxicity $\geq$ grade $2(p=0.010)$, also tumor stage IIIA $(p<0.001)$, tumor stage IIIB $(p=0.003)$, gender $(p=0.042)$ and fatigue $(p<0.001)$ appeared to be significant predictors of QoL. WHO performance status, chemotherapy, age, and histology did not significantly influence the changes in QoL over time

Conclusion: High dose radiotherapy or concurrent chemo-radiation in the treatment of lung cancer seems to be a well tolerated treatment option with preservation of QoL. 


\section{INTRODUCTION}

Lung cancer remains a major public health problem worldwide because of its high incidence, rapid progression and poor outcome ${ }^{1}$. The majority of patients who develop lung cancer die of this disease within a year ${ }^{2}$. Aggressive radiotherapy or concurrent chemo-radiation therapy for lung cancer is standard care for patients with locally advanced disease, i.e. stage III non-small cell lung cancer (NSCLC) or limited disease small cell lung carcinoma (LD-SCLC) $)^{3,4}$.

Combined concurrent modality treatment increases long-term survival, but at the expense of a higher incidence of severe esophagitis ${ }^{5-8}$. In order to allow proper balancing between expected benefits and drawbacks of aggressive therapy, knowledge of the effects of a given treatment on the quality of life (QoL) is needed.

An optimal therapy could be defined as one that increases survival or provides benefit through reduction of cancer-related symptoms and improved QoL.

To the best of our knowledge, in only one study, QoL assessment was performed in patients receiving high-dose radiotherapy for NSCLC ${ }^{9}$. No data are at present known on the influence of concurrent chemo-radiation on the QoL.

Moreover, a Medline search using the terms "QoL and SCLC" (April 2008) revealed only 12 studies of which none examined QoL after radiotherapy treatment.

The purpose of this study was to investigate the evolution of QoL on NSCLC stage IIII and LD-SCLC patients, referred for hyperfractionated accelerated high-dose radiotherapy (RT) with or without concurrent chemotherapy, with curative intent. Since we hypothesized that QoL would be considerably influenced by esophageal toxicity, we specifically studied the relationship between QoL and esophageal toxicity.

\section{PATIENTS EN METHODS}

\subsection{Study population and design}

We used a longitudinal design to assess different aspects of symptoms and functioning regarding QoL in lung cancer patients treated with high dose radiotherapy. Patients with small cell lung carcinoma (SCLC) and patients with non-small cell lung carcinoma (NSCLC) selected for curative treatment with high-dose radiotherapy, were asked to participate in this study.

The entry criteria were as follows: cytologically or histologically proven NSCLC or LDSCLC with the exclusion of mixed pathology between NSCLC and small-cell cancer and bronchioloalveolar carcinoma; UICC stage I-III, with the exclusion of T4 lesions in case of malignant pleural effusion; WHO performance status 0-3; measurable disease; age at least 18 years; adequate pulmonary function (FEV1>1liter); no severe recent cardiac disease (arrhythmia, congestive heart failure, infarction); able to com- 
ply with the protocol; able to give written informed consent. Distant metastases had to be absent both on CT and on whole-body ${ }^{18} \mathrm{~F}$-deoxyglucose (FDG)-PET scan. In stage III NSCLC or LD-SCLC, a CT or an MRI scan of the brain was performed to rule out asymptomatic brain metastases.

To assess the QoL, patients were asked to complete the European Organization for Research and Treatment of Cancer Quality of life Questionnaire (EORTC QLQ-C30) with a lung cancer module (EORTC QLQ-LC13). The questionnaire was completed at baseline (before radiotherapy) and 2, 6 weeks and 3, 6, 9, 12, and 18 months after radiotherapy. Additionally, at each time point, standard toxicity scores were registered by the radiation-oncologist. Pulmonary and esophageal toxicity were scored according to the EORTC/RTOG criteria for acute or late side effects ${ }^{10,11}$.

\subsection{Ethics}

The local Medical Ethical Committee approved the protocol in accordance with Dutch law and regulations. All patients gave written informed consent before the start of the treatment.

\subsection{Measurement instruments}

Background variables included age, gender, tumor staging, and the WHO performance level. The EORTC Quality of Life Questionnaire (QLQ-C30, version 3.0) is a 30item cancer-specific core questionnaire that addresses various domains of $\mathrm{QoL}^{12}$. It contains five function scales (physical functioning, role functioning, emotional functioning, cognitive functioning and social functioning), three symptom scales (fatigue, pain and nausea/vomiting), two items assessing global health and quality of life, and a number of single items addressing various symptoms and perceived financial impact. The additional Lung cancer module (EORTC QLQ-LC 13) contains 13 items concerning specific symptoms with regard to lung cancer ${ }^{13}$.

\subsection{Treatment}

\subsubsection{Radiotherapy}

Radiotherapy planning was performed with a Focal (Computerized Medical Systems, Inc ${ }^{\mathrm{TM}}$ ) treatment planning system, using inhomogeneity corrections based on a convolution algorithm. For the delivery of the radiotherapy treatment, multiple photon fields from a 6-10-MV linear accelerator were used. Dose specification was done according to ICRU 50 guidelines ICRU ${ }^{14}$.

For NSCLC, three dose-escalation groups were defined, according to the risk of severe radiation pneumonitis based on the $\mathrm{V}_{20 \mathrm{~Gy}}{ }^{15}$. As in RTOG 93-11, three risk 
groups were identified: $\mathrm{V}_{20 \mathrm{~Gy}}<25 \%, \mathrm{~V}_{20 \mathrm{~Gy}} 25-37 \%$, and $\mathrm{V}_{20 \mathrm{~Gy}}>37 \%{ }^{15}$. For each risk group a 3D conformal treatment plan was calculated. The dose was administered in three steps: $61.2 \mathrm{~Gy} / 34$ fractions/23 days, $64.8 \mathrm{~Gy} / 36$ fractions/24 days and 68.40 Gy/38 fractions/25 days (all schedules used $1.8 \mathrm{~Gy}$ BID with $8 \mathrm{~h}$ interval), according to ICRU 50 guidelines ${ }^{14}$. More detailed information can be found in an earlier publication ${ }^{16}$.

For LD-SCLC, a 3D conformal treatment plan was calculated, with a prescribed dose to the PTV of $45 \mathrm{~Gy}$ in 30 fractions in 3 weeks (1.5 Gy BID, 6-8h interval), according to ICRU 50 guidelines ${ }^{14}$. More detailed information can be found in an earlier publication ${ }^{17}$.

\subsubsection{Chemotherapy}

Chemotherapy cycles were repeated every 21 days for a total of 3 (NSCLC) or 5 cycles (LD-SCLC). The carboplatin dose (in milligrams) was based on the target area under the curve $(A \cup C)(5 \mathrm{mg} / \mathrm{ml} / \mathrm{min}) \times$ (glomerular filtration rate +25$)$, with the glomerular filtration rate calculated according to the Cockroft-Gault formula.

For NSCLC, in cases where induction chemotherapy was administered, patients received carboplatin (AUC $5 \mathrm{mg} / \mathrm{ml} / \mathrm{min}$ ) on day 1 and gemcitabine $1250 \mathrm{mg} / \mathrm{m}^{2}$ on day 1 and 8 , according to the standard protocol of the Comprehensive Cancer Center Limburg, the Netherlands. Standard dose-reduction rules were applied if indicated. No patient received concurrent chemotherapy and radiotherapy. Radiotherapy was initiated not earlier than 14 days after the last gemcitabin administration, and no later than 21 days after the last chemotherapy delivery.

All LD-SCLC patients received carboplatin (AUC $5 \mathrm{mg} / \mathrm{ml} / \mathrm{min}$ ) on day 1 and etoposide $120 \mathrm{mg} / \mathrm{m}^{2}$ on day 1,2 , and 3 per cycle. Standard dose-reduction rules were applied if indicated. Chest radiation started after a mean time of 17.7 days \pm 9.7 days Standard Deviation (SD) after the beginning of chemotherapy.

\subsection{Statistical analysis}

Descriptive statistics were calculated for demographics and treatment characteristics. Changes in symptom and QoL scores over time (days after start of treatment) were evaluated with a mixed models linear regression analysis with time as the only independent variable and an unstructured repeated measures covariance type, using SPSS software (version 15.0).

Mixed linear regression models were fitted to investigate the impact of different factors (and especially esophagus toxicity) on overall QoL (item 30 on the EORTC QLQC30). As independent variables, chemotherapy (yes/no), WHO performance status (2 
dummies: $\mathrm{WHO} 0=$ reference, $\mathrm{WHO} 1=$ dummy $1, \mathrm{WHO} 2=$ dummy 2 ), tumor stage (2 dummies: $\leq$ stage $I I=$ reference, stage $I I I A=$ dummy 1 , stage $I I I B=$ dummy 2 ) and maximal esophagus toxicity ( 2 dummies: no tox.= reference, grade $1=$ dummy 1 , grade $\geq 2$ = dummy 2) were included. The dependent variable in these regression analyses was overall QoL (EORTC QLQ-30, item 30); age, gender and histology (SCLC/NSCLC), were included as potential confounders. Since fatigue is the most frequently reported cancer related side-effect, being of significant influence on QoL, we also adjusted for this variable ${ }^{18-20}$.

The following steps were taken to attain a model that fitted the data best. First, exploratory analyses were carried out by visual inspection of individual and mean patient profiles to provide information on the structural form (i.e. random intercept, random slope, variability in variance, linear relationships and possible interaction) and through scatter plot and correlation matrices of residuals obtained from ordinary least squares (OLS) regression which provide information on the correlation or covariance structure. Second, a full linear model was formed based on the exploratory analyses and several covariance structures were compared using the log likelihood ratio test. Restricted Maximum Likelihood (REML) estimation methods were used during this step. The model with the least parameters to be estimated was preferred, unless the log likelihood ratio test showed that addition of more parameters improved the model significantly. This procedure was repeated until addition of extra parameters did not improve the model anymore. The different models that were compared included a random intercept on the patient level with covariance types Unstructured, Toeplitz heterogeneous, Toeplitz, Compound Symmetry and Scaled Identity, or no random components with covariance structures Unstructured, Toeplitz heterogeneous, and Unstructured. Finally, after the best covariance structure was chosen, the model was then reduced using a top-down procedure and the Maximum Likelihood (ML) estimation method. The primary predictor (esophagus toxicity) and possible confounders (gender, age and histology) always remained in the model.

All results were regarded as statistically significant if the probability of chance was $5 \%$ or less ( $p$-value $<0.05$ ).

\section{RESULTS}

\subsection{Patient characteristics}

Patient characteristics are depicted in Table 1. The studied dataset contained 75 patients, of which 19 (25\%) were women; 45 patients (60\%) had NSCLC and 30 $(40 \%)$ had SCLC. The mean age of the total group was 67 years (SD 8.8). At 18 months after radiotherapy 45 patients $(60 \%)$ were alive and without recurrence of disease. 
Table 1: Demographics of 75 patients undergoing high dose curative radiotherapy for lung cancer.

\begin{tabular}{|c|c|c|c|c|}
\hline & & $\begin{array}{l}\text { NSCLC } \\
\%(n=45)\end{array}$ & $\begin{array}{l}\text { SCLC } \\
\%(n=30)\end{array}$ & $\begin{array}{l}\text { Total group } \\
\%(n=75)\end{array}$ \\
\hline \multicolumn{5}{|l|}{ Gender } \\
\hline & Male & 64 & 36 & 75 \\
\hline & Female & 47 & 53 & 25 \\
\hline \multicolumn{5}{|l|}{ Age (years) } \\
\hline & mean & 69 & 64 & 67 \\
\hline & S.D. & 8.1 & 9.0 & 8.8 \\
\hline & range & $(54-89)$ & $(47-85)$ & $47-89$ \\
\hline \multicolumn{5}{|c|}{ Compliance * (population alive) } \\
\hline & Baseline & $84(60)$ & $87(40)$ & $85(100)$ \\
\hline & 2 weeks & $93(60)$ & $10(40)$ & $60(100)$ \\
\hline & 6 weeks & $89(60)$ & $100(40)$ & $93(100)$ \\
\hline & 3 months & $91(59)$ & $97(41)$ & $93(97)$ \\
\hline & 6 months & $97(56)$ & $79(44)$ & $89(85)$ \\
\hline & 9 month & $85(59)$ & $79(41)$ & $83(77)$ \\
\hline & 12 months & $90(57)$ & $74(43)$ & $83(72)$ \\
\hline & 18 months & $88(56)$ & $55(44)$ & $73(60)$ \\
\hline \multicolumn{5}{|l|}{ Tumor stage } \\
\hline & $|-| \mid$ & 23 & 1 & 24 \\
\hline & IIIA & 19 & 9 & 28 \\
\hline & IIIB & 19 & 24 & 43 \\
\hline & LD & 0 & 5 & 5 \\
\hline \multicolumn{5}{|l|}{ WHO } \\
\hline & 0 & 36 & 17 & 53 \\
\hline & 1 & 23 & 13 & 36 \\
\hline & 2 & 1 & 4 & 5 \\
\hline & 3 & 0 & 1 & 1 \\
\hline & unknown & 0 & 5 & 5 \\
\hline \multirow[t]{5}{*}{ Radiotherapy } & TTD: & & & \\
\hline & 61,2 Gy/1,8GyBID & 33 & 0 & 33 \\
\hline & 64,8 Gy/1,8GyBID & 19 & 0 & 19 \\
\hline & 68,4 Gy/1,8GyBID & 8 & 0 & 8 \\
\hline & 45Gy/1,5GyBID & 0 & 40 & 40 \\
\hline \multicolumn{5}{|l|}{ Chemotherapy } \\
\hline & yes & 29 & 40 & 69 \\
\hline & no & 31 & 0 & 31 \\
\hline
\end{tabular}

Values are percentages unless stated otherwise.

Abbreviations: NSCLC $=$ non small cell lung cancer; SCLC=small cell lung cancer; S.D.=standard deviation; $\mathrm{LD}=$ limited disease; $\mathrm{WHO}=$ world health organization; $\mathrm{TTD}=$ total tumor dose; $\mathrm{Gy}=\mathrm{gray} ; \mathrm{BID}=$ twice daily

\subsection{Compliance with QoL assessment}

At baseline (before start radiotherapy) $85 \%$ of the patients $(n=64)$ completed the questionnaire (Table 1). After radiotherapy the compliance rates were $60 \%$ at 2 weeks (45 of 75 patients alive), 93\% at 6 weeks (70 of 75 patients alive), 93\% at 3 months (68 out of 73 patients alive), $89 \%$ at 6 months (57 out of 64 patients alive), $83 \%$ at 9 months (48 of 58 patients alive), $83 \%$ at 12 months (45 out of 54 patients alive) and $73 \%$ at 18 months (33 out of 45 patients alive). 


\subsection{Evolution of QoL, functioning, and symptom scores over time}

Mean baseline disease-related symptom QoL scores and mean changes from baseline are depicted in Table 2.

QoL decreased short after the end of RT $\left(\Delta \mathrm{T}_{0}-\mathrm{T}_{2}\right.$ : mean $\left.=-40\right)$ but increased back to baseline within 3 months $\left(\Delta T_{0}-T_{3}\right.$ : mean $\left.=3.5\right)$, and remained relatively stable thereafter. None of these changes over time are significant $(p=0.943)$. Mean scores of role functioning $(p=0.018)$, cognitive functioning $(p=0.002)$, dyspnoea (EORTC QLQLC13; $p=0.043)$, dysphagia $(p=0.005)$ and hoarseness $(p=0.029)$, showed a significant worsening over time. Emotional functioning $(p=0.033)$ improved significantly over time. The course over time of QoL and these variables are depicted in Figure $1 \mathrm{a}-\mathrm{g}$. Although the changes in all functioning and symptoms depicted in Figure $1 \mathrm{~b}-\mathrm{g}$ were statistical significant, the magnitude of these effects was small (regression coefficients in absolute terms ranging from 0.009 to 0.107 ). The remaining symptoms and functioning scores were stable over time.

\subsection{The role of esophageal toxicity on QoL}

Out of 75 patients, maximal esophageal toxicity could be calculated for 61 patients (31 grade 0, 21 grade 1, and $9 \geq$ grade 2). As hypothesized, esophageal toxicity significantly influenced $\mathrm{QoL}$, however only if $\geq$ grade 2 . Next to maximal esophageal toxicity $\geq$ grade $2(p=.0 .010)$, also tumor stage IIIA $(p<0.001)$, tumor stage IIIB $(p=0.003)$, gender $(p=0.042)$ and fatigue $(p<0.001)$ appeared to be significant predictors of QoL (Table 3). WHO performance status, chemotherapy, age, and histology did not significantly influence the changes in QoL over time.

Table 3: Estimates from linear regression analysis of fixed effects on QoL.

\begin{tabular}{|c|c|c|c|c|c|}
\hline Parameter & Estimate & $95 \% \mathrm{Cl}$ & & Std. error & p-value \\
\hline Gender & -6.14 & -12.07 & -0.22 & 2.95 & 0.042 \\
\hline Histology & & & & & ns \\
\hline Age & & & & & ns \\
\hline Fatigue & -0.42 & -0.48 & -0.35 & 0.03 & $<0.001$ \\
\hline \multicolumn{6}{|l|}{ Tumor stage* } \\
\hline Stage III A & 15.92 & 8.72 & 23.13 & 3.58 & $<0.001$ \\
\hline Stage III B & 10.95 & 4.03 & 17.88 & 3.42 & 0.003 \\
\hline \multicolumn{6}{|c|}{ Maximal esophageal toxicity* } \\
\hline Grade I & & & & & ns \\
\hline Grade $\geq$ II & -10.76 & -18.91 & -2.61 & 4.08 & 0.010 \\
\hline
\end{tabular}

Dependent variable: item 30 EORTC QLQ-C30, adjusted for age, gender and histology

Chemotherapy and WHO performance status were not significant

* reference category for tumor stage $=$ stage $\leq \mathrm{II}$, and for maximal esophagus tox. $=$ no tox. (grade 0 )

ns= not significant 


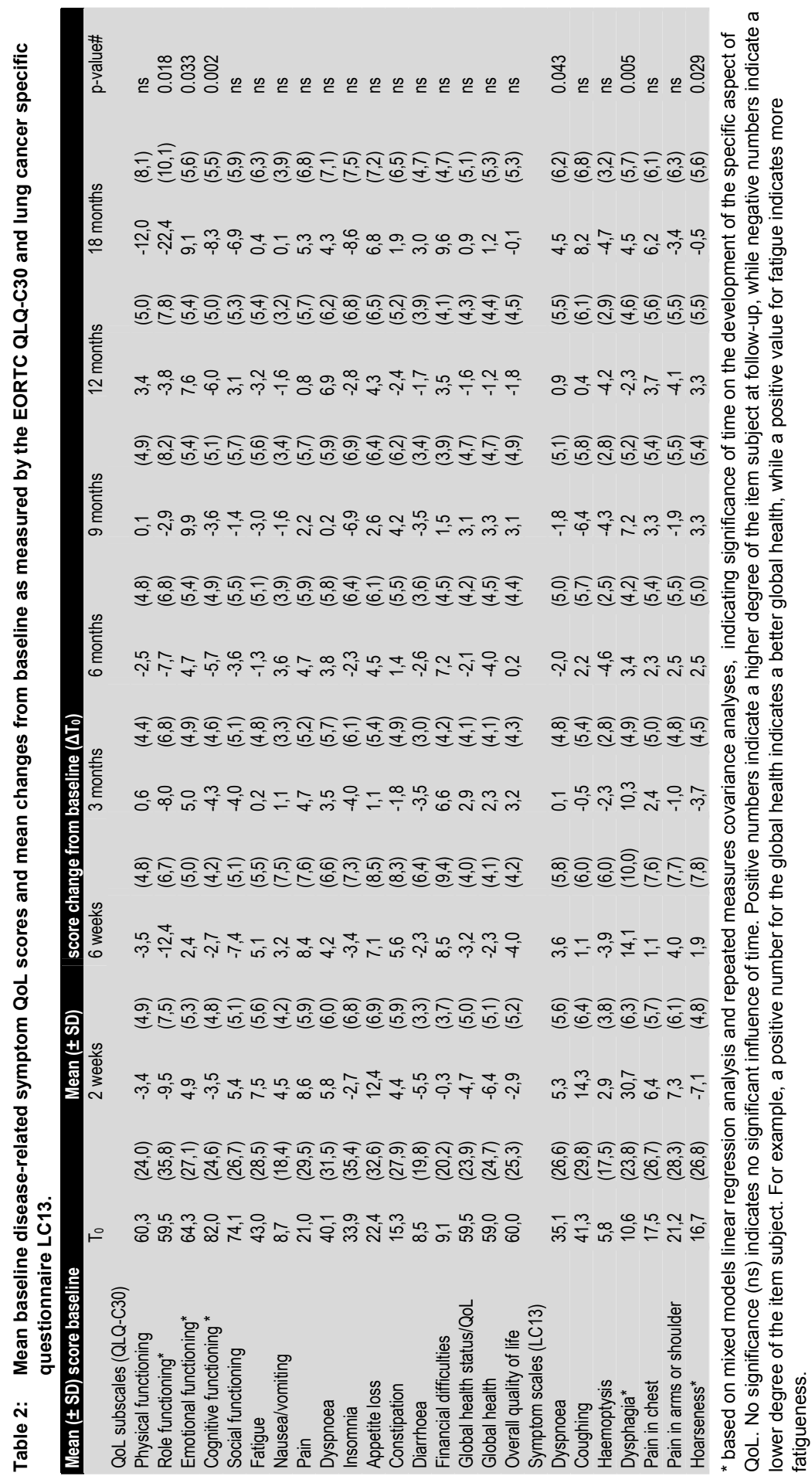




\section{2 | Part 2: QoL- Chapter 6}

Figure 1: Evolution of QoL (1a) and evolution of functioning and symptoms which showed significant changes in time $(1 \mathrm{~b}-\mathrm{g})$
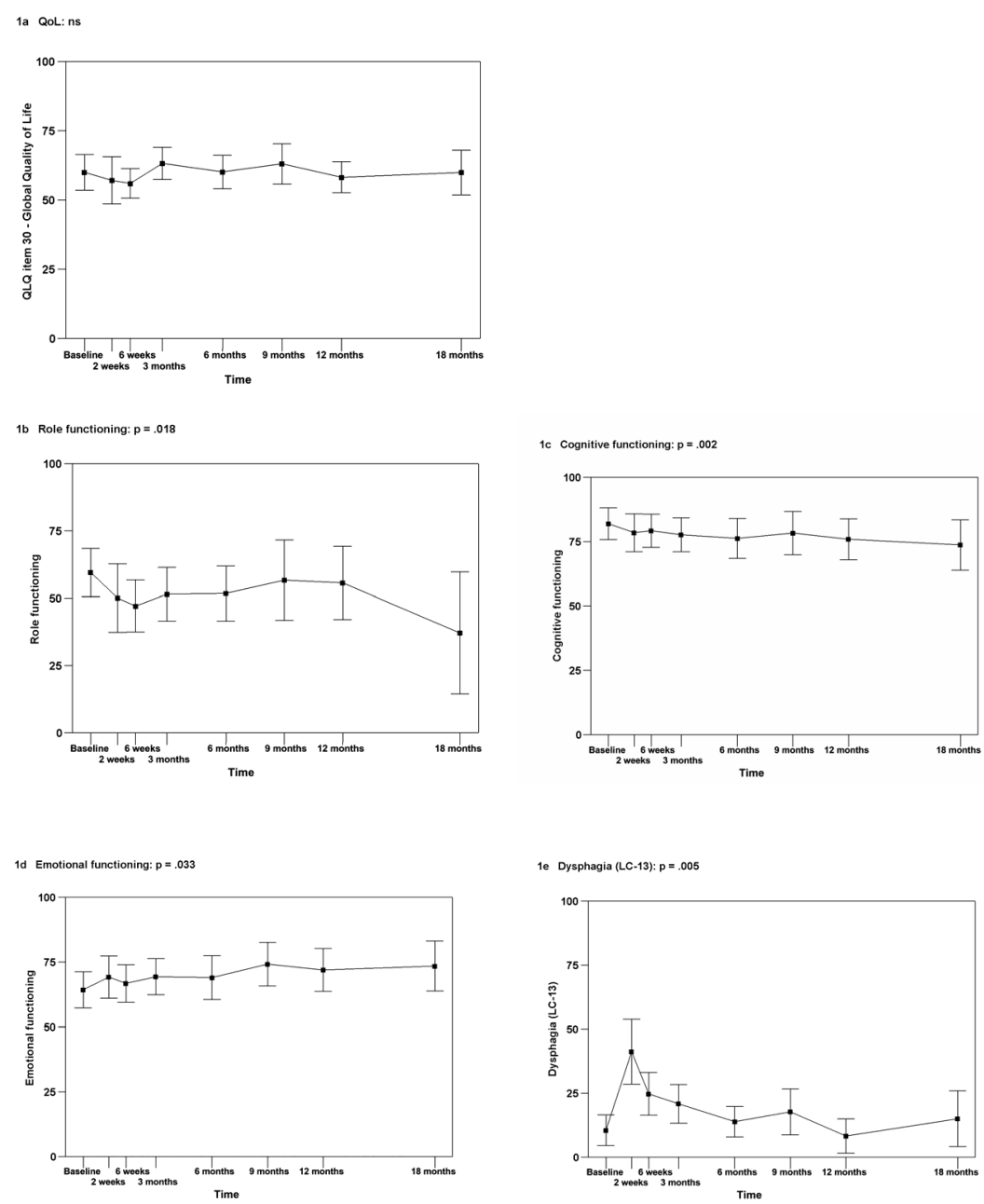

1f Dyspnoea (LC-13): $\mathrm{p}=.033$
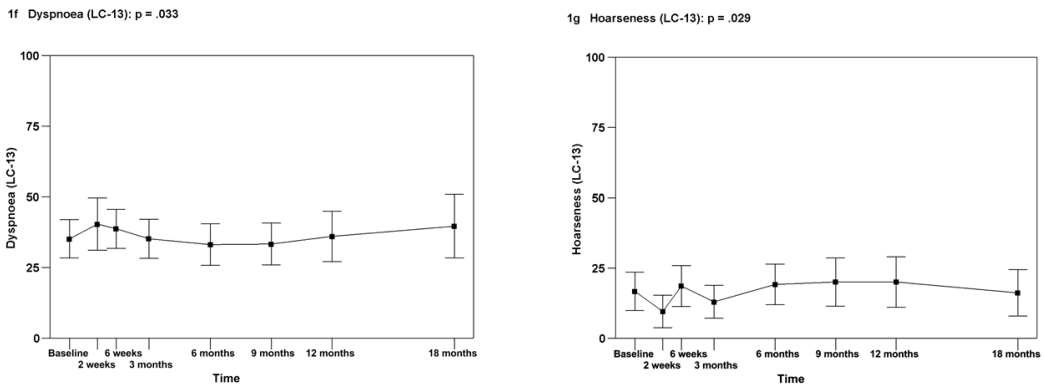

Time 


\section{Discussion}

To our knowledge, this is the first prospective QoL study on lung cancer treated with high-dose radiotherapy with or without chemotherapy, including patients with different stages and histology. The only previous study to report on QoL issues after treatment with an accelerated radiotherapy regimen was carried out by Auchter et al $^{9}$, who investigated QoL in 30 NSCLC patients, all stage III. In their conclusion they reported that this aggressive approach did not cause a significant, long-term decrease in the QoL of the treated patients.

In the current study, these results above were confirmed.

QoL decreased shortly after RT but increased again within 3 months after the end of RT. This short decrease of global quality of life could be explained by the side effects of RT. In addition, the majority of the symptoms and functioning scores did not change much over time, and if so, it was reversible, and occurred only shortly after finishing radiotherapy. However, in some symptoms and functioning scores, a persistent worsening was seen: Physical state, accomplishing daily tasks (role) and cognitive functioning, dyspnea, dysphagia, and hoarseness, detoriated after RT, and were significantly worse at the end of the follow-up period. Conversely, as also reported in a previous study ${ }^{9}$, emotional functioning scores were significantly higher at the end of the follow-up period as compared to baseline.

Histology (SCLC/NSCLC), WHO performance status and additional treatment with chemotherapy did not influence the overall QoL.

Adjusted for potential confounders, esophagitis appeared to be a strong predictor for QoL. However, the number of patients who suffer from severe esophagitis appeared to be very low. A substantial decrease of QoL after high dose RT in the current study population did only occur in a very small proportion of patients (12\%). More apparent predictors for QoL found in this study appeared to be gender, tumor stage, and fatigue. This latter finding is not surprising, as fatigue is identified as the most predominant factor that influences overall QoL ${ }^{18,19,21}$. In addition, the more persistent worsening of some symptoms and functioning scores may be caused by the occurrence of relapse. However, since we did not take this into account in our analysis we are not able to draw any conclusion on this.

Our results showed that performance status (PS) did not significantly contributed to the overall Qol. Earlier publications that reported on the correlation between PS and QoL are ambiguous ${ }^{18,22,23}$. It could be, since we adjusted for fatique, a variable which is identified as a strong potential confouder, that the PS pales into insignificance. Another reason why PS didn't correlate with QoL in the present study could be explained the relatively good condition of the study population. The main group (53\%) had a very good of PS (WHO PS= 0 ). 
We found that the course of QoL over time distincted between men and women. From baseline until 18 months after RT, in females their mean QoL scores were improved from 48.8 (SD 26.5) to 64.3 (SD 22.3), wereas in males their QoL scores decreased from 63.2 (SD 24.3) to 58.7 (SD 22.6) in the same period. This finding is in agreement with two recent publications ${ }^{22,24}$. Using the Functional Assessment of Cancer Therapy [FACT], Movsas et $\mathrm{al}^{24}$ observed pretreatment a clinically meaningful difference in lung cancer patients between males (FACT 87.3) and females (FACT 78.6). Siddiqui et $\mathrm{al}^{22}$ focused in a prospective randomized lung cancer trial on gender-specific baseline health-related QOL and Karnofsky performance score (KPS). They reported significant KPS-by-gender interactions in the physical well-being and additional concerns-lung subscales. Elaborating on this result, it is probable that the persistent decrease in role functioning noted in the current study, is associated with gender.

In the present study tumor stage appeared also to be associated with QoL. Patients with stage III, regardless of histology, reported a less variation of QoL over time as compared to patients with stage I-II. A trend towards higher baseline QoL scores for patients with stage III as compared to stage I-II (48.95 \pm 26.9 vs. $63.89 \pm 24.9, p=$ 0.051 ) was found. The lower QoL scores in stage I-II patients as compared to stage III patients could be explained by the fact that the main part of the stage I-II patients were referred for RT because they were medically inoperable due to a worse physical condition and/or co-morbidities. Correspondingly, Manser et $\mathrm{al}^{25}$ found higher utility scores on stage III NSCLC as compared to stage I-II, taken into account the proportion of operability and co-morbidities.

A gradual worsening was observed for dysphagia, dyspnea and hoarseness. Although we did not correct for the natural history of chronic obstructive pulmonary disease $^{26,27}$ that would also explain increasing dyspnea in time, in which radiationinduced lung damage could obviously also play a role.

High dose radiotherapy or concurrent chemo-radiation in the treatment of lung cancer seems to be a well tolerated treatment option with preservation of QoL.

After fatigue, acute esophagitis had the largest impact on QoL. In extension to this result, it is recommended when implementing new innovations in $\mathrm{RT}^{28,29}$ such as intensity-modulated RT, tomotherapy, stereotactic RT or particle therapy should carefully monitor the QoL of patients, in order to evaluate this prognostic factor and the impact of the new technology. 


\section{REFERENCES}

1. Shibuya K, Mathers CD, Boschi-Pinto C, et al: Global and regional estimates of cancer mortality and incidence by site: II. Results for the global burden of disease 2000. BMC Cancer 2:37, 2002

2. Ferlay J, Autier P, Boniol M, et al: Estimates of the cancer incidence and mortality in Europe in 2006. Annals of oncology 18:581-92, 2007

3. Auperin A, Rolland E, Curran WJ: Concomitant radio-chemotherapy (RT-CT) versus sequential RT-CT in locally advanced non-small cell lung cancer (NSCLC): A meta-analysis using individual patient data (IPD) from randomised clinical trials (RCTs). J Thor Oncol 2:S310 (Abstr A1-05), 2007

4. Turrisi AT, 3rd, Kim K, Blum R, et al: Twice-daily compared with once-daily thoracic radiotherapy in limited small-cell lung cancer treated concurrently with cisplatin and etoposide. N Engl J Med 340:26571, 1999

5. Zatloukal P, Petruzelka L, Zemanova M, et al: Concurrent versus sequential chemoradiotherapy with cisplatin and vinorelbine in locally advanced non-small cell lung cancer: a randomized study. Lung Cancer 46:87-98, 2004

6. Fournel P, Robinet G, Thomas $\mathrm{P}$, et al: Randomized phase III trial of sequential chemoradiotherapy compared with concurrent chemoradiotherapy in locally advanced non-small-cell lung cancer: Groupe Lyon-Saint-Etienne d'Oncologie Thoracique-Groupe Francais de Pneumo-Cancerologie NPC 95-01 Study. J Clin Oncol 23:5910-7, 2005

7. Furuse K, Fukuoka M, Kawahara M, et al: Phase III study of concurrent versus sequential thoracic radiotherapy in combination with mitomycin, vindesine, and cisplatin in unresectable stage III nonsmall-cell lung cancer. J Clin Oncol 17:2692-9, 1999

8. Vokes EE, Crawford J, Bogart J, et al: Concurrent chemoradiotherapy for unresectable stage III nonsmall cell lung cancer. Clin Cancer Res 11:5045s-5050s, 2005

9. Auchter RM, Scholtens D, Adak S, et al: Quality of life assessment in advanced non-small-cell lung cancer patients undergoing an accelerated radiotherapy regimen: report of ECOG study 4593. Eastern Cooperative Oncology Group. Int J Radiat Oncol Biol Phys 50:1199-206, 2001

10. Rubin P, Constine LS, 3rd, Fajardo LF, et al: EORTC Late Effects Working Group. Overview of late effects normal tissues (LENT) scoring system. Radiother Oncol 35:9-10, 1995

11. Rubin P, Constine LS, Fajardo LF, et al: RTOG Late Effects Working Group. Overview. Late Effects of Normal Tissues (LENT) scoring system. Int J Radiat Oncol Biol Phys 31:1041-2, 1995

12. McLachlan S-A, Devins GM, Godwin PJ: Validation of the European Organisation for Research and Treatment of Cancer quality of life questionaire (QLQ-C30) as a measure of psychosocial function in breast cancer patients. European journal of cancer 34:510-517, 1998

13. Bergman B, Aaronson NK, Ahmedzai S, et al: The EORTC QLQ-LC13: a modular supplement to the EORTC Core Quality of Life Questionnaire (QLQ-C30) for use in lung cancer clinical trials. EORTC Study Group on Quality of Life. Eur J Cancer 30A:635-42, 1994

14. ICRU: International Commission on radation Units and Measurements report 50. Prescribing, recording and reporting photon beam therapy, Oxford University Press. Oxford, 1993

15. Bradley J, Graham MV, Winter K, et al: Toxicity and outcome results of RTOG 9311: a phase I-II doseescalation study using three-dimensional conformal radiotherapy in patients with inoperable non-smallcell lung carcinoma. Int J Radiat Oncol Biol Phys 61:318-28, 2005

16. De Ruysscher D, Wanders R, van Haren E, et al: HI-CHART: A Phase I/II Study on the Feasibility of High-Dose Continuous Hyperfractionated Accelerated Radiotherapy in Patients with Inoperable NonSmall-Cell Lung Cancer. Int J Radiat Oncol Biol Phys, 2007

17. De Ruysscher D, Bremer RH, Koppe F, et al: Omission of elective node irradiation on basis of CTscans in patients with limited disease small cell lung cancer: a phase II trial. Radiother Oncol 80:30712, 2006

18. Dagnelie PC, Pijls-Johannesma MC, Lambin P, et al: Impact of fatigue on overall quality of life in lung and breast cancer patients selected for high-dose radiotherapy. Ann Oncol 18:940-4, 2007 


\section{6 | Part 2: QoL- Chapter 6}

19. Langendijk JA, Aaronson NK, de Jong JM, et al: Prospective study on quality of life before and after radical radiotherapy in non-small-cell lung cancer. J Clin Oncol 19:2123-33, 2001

20. Langendijk JA, Aaronson NK, de Jong JM, et al: Quality of life after curative radiotherapy in Stage I non-small-cell lung cancer. Int J Radiat Oncol Biol Phys 53:847-53, 2002

21. Langendijk JA, Aaronson NK, ten Velde GP, et al: Pretreatment quality of life of inoperable non-small cell lung cancer patients referred for primary radiotherapy. Acta Oncol 39:949-58, 2000

22. Movsas B, Scott C, Watkins-Bruner D: Pretreatment factors significantly influence quality of life in cancer patients: a Radiation Therapy Oncology Group (RTOG) analysis. Int J Radiat Oncol Biol Phys 65:830-5, 2006

23. Siddiqui $\mathrm{F}$, Kohl R, Swann S, et al: Gender differences in pretreatment quality of life in a prospective lung cancer trial. J Support Oncol 6:33-9, 2008

24. Manser RL, Wright G, Byrnes G, et al: Validity of the Assessment of Quality of Life (AQoL) utility instrument in patients with operable and inoperable lung cancer. Lung Cancer 53:217-29, 2006

25. Lange $\mathrm{P}$, Parner J, Vestbo J, et al: A 15-year follow-up study of ventilatory function in adults with asthma. N Engl J Med 339:1194-200, 1998

26. Scharloo M, Kaptein AA, Weinman JA, et al: Physical and psychological correlates of functioning in patients with chronic obstructive pulmonary disease. J Asthma 37:17-29, 2000 


\section{PART 3}

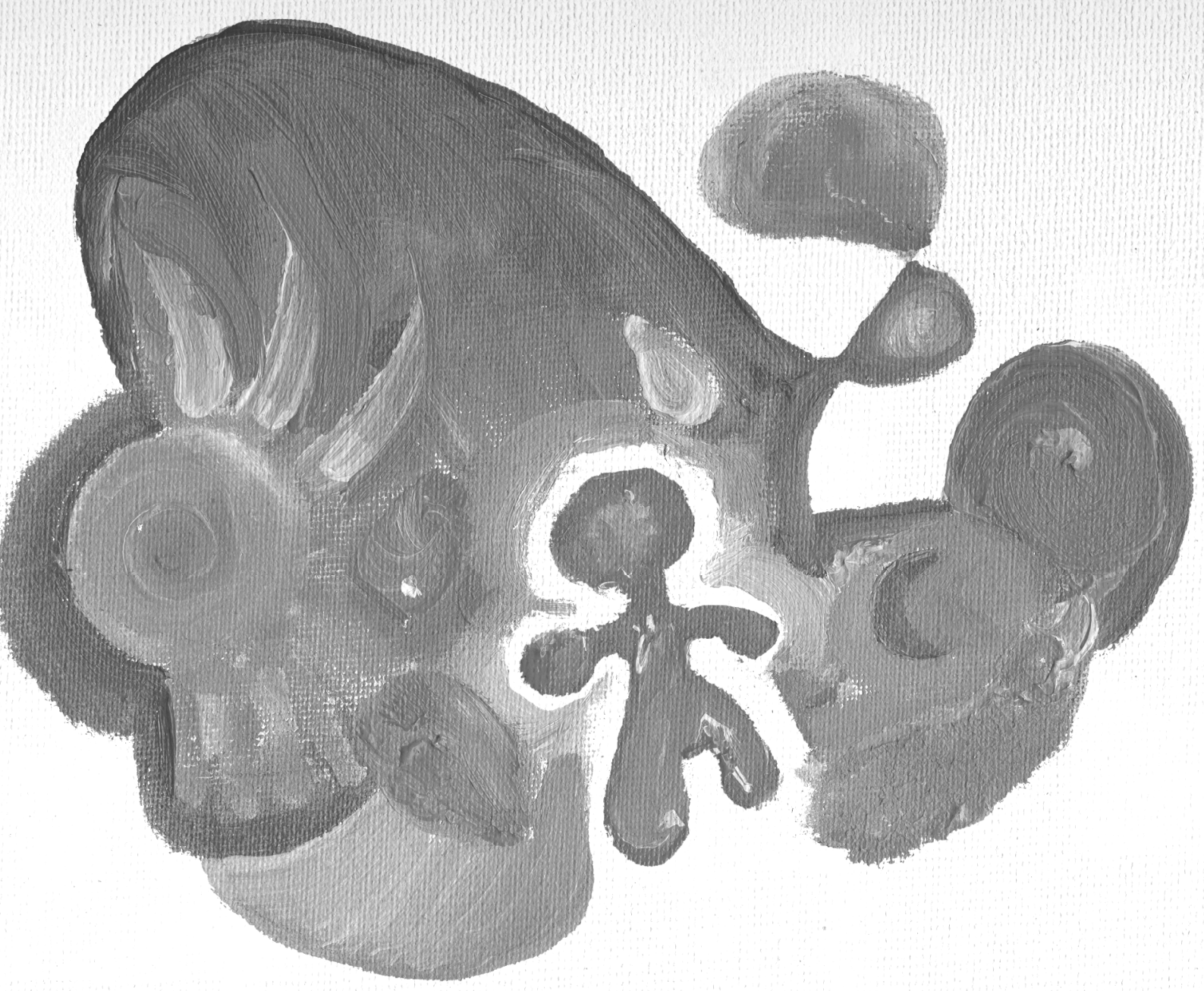




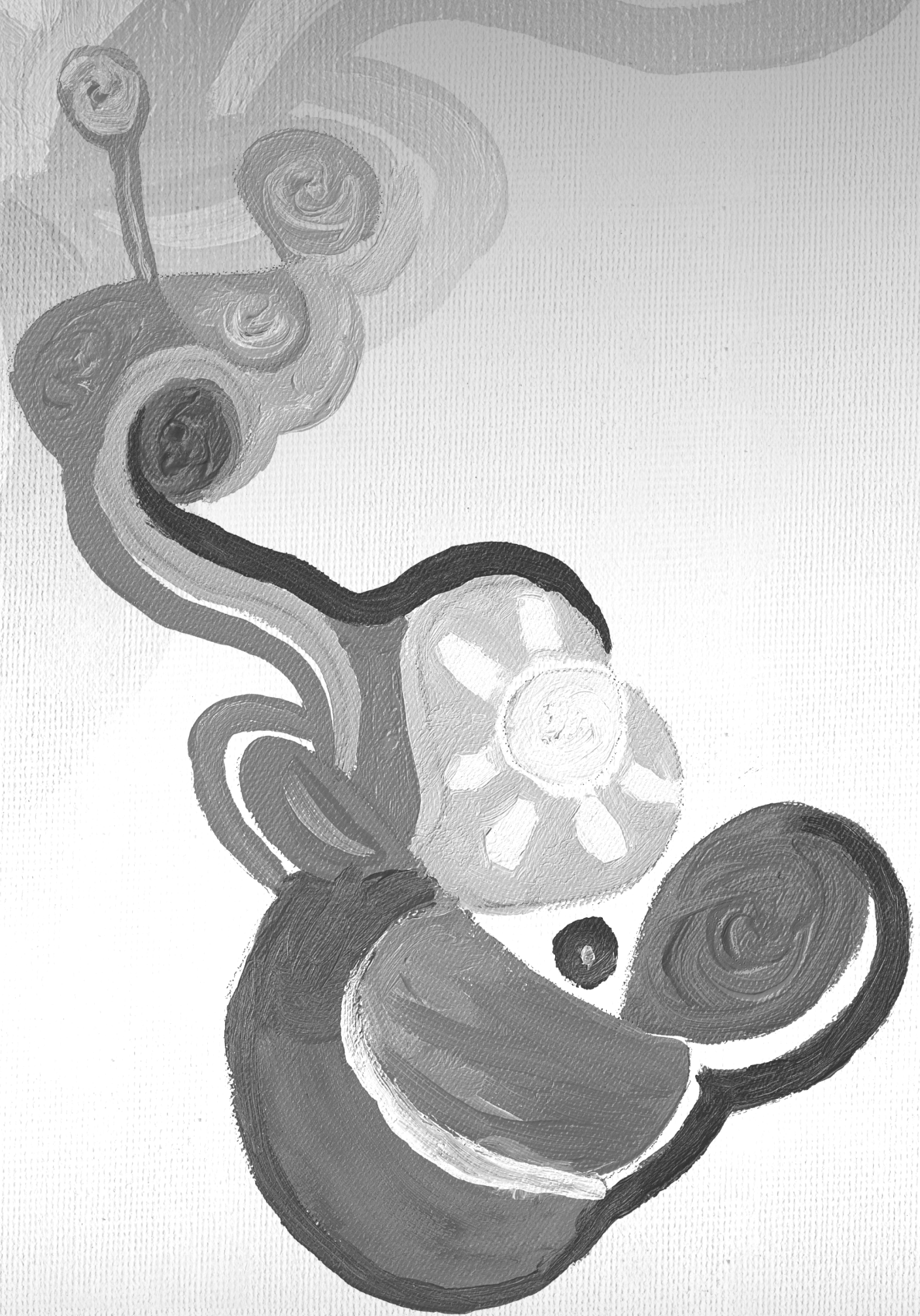




\section{CHAPTER}

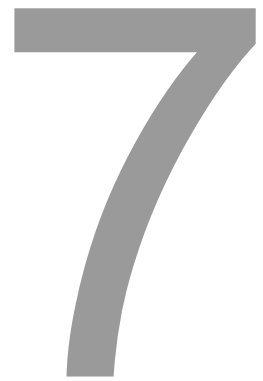

\section{Particle therapy in lung cancer: Were do we stand? ${ }^{1}$}

Cancer Treatment Reviews (2008), 34: 259-67

Madelon Pijls-Johannesma Janneke Grutters

Philippe Lambin

Dirk De Ruysscher

\footnotetext{
1 This chapter is modified, also parts of the below references were included:

- Brada M, Pijls-Johannesma M, De Ruysscher D: Proton therapy in clinical practice: current clinical evidence. J Clin Oncol (2007), 25:965-970.

- Lodge M, Pijls-Johannesma M, Stirk L, Owens N, Munro A, De Ruysscher D, Jefferson T: A systematic literature review of the clinical and cost effectiveness of hadron therapy in cancer. Radiother Oncol (2007), 83:110-22, .
} 


\section{ABSTRACT}

Background: From a theoretical point of view, charged particles should lead to superior results compared to photons. In this review, we searched for clinical evidence that protons or $\mathrm{C}$-ions are really beneficial to patients with lung cancer.

Methods: A systematic literature review based on an earlier published comprehensive review was performed and updated until November ${ }^{\text {st }} 2007$.

Results: Ten fully published series, all dealing with non-small cell lung cancer (NSCLC), mainly stage I, were identified. No phase III trials were found. On proton therapy, 2-5 year local tumor control rates varied between $87 \%$ and $57 \%$. The 2 year $/ 5$ year overall survival and 2 year/5 year cause specific survival varied between $31-74 \% / 23 \%$ and $58-86 \% / 46 \%$ respectively. Late side effects were observed in about $10 \%$ of the patients. For C-ion therapy, the local tumor control rate was $77 \%$, while $95 \%$ when using a hypofractionated radiation schedule. The 5 year overall survival and cause specific survival rates were $42 \%$ and $60 \%$ respectively. Slightly better results were reported when using hypofractionation, $50 \%$ and $76 \%$ respectively. The reported late side effects were $4 \%$.

Conclusion: The results with charged particles, at least for stage I disease, seem to be promising. A gain can be expected in reduction of late side effects, especially after treatment with $\mathrm{C}$-ions. Available data demonstrate that particle therapy in general is a safe and feasible treatment modality. Although current results are promising, more evidence is required before particle therapy can become internationally the standard treatment for (subsets of) lung cancer patients. 


\section{INTRODUCTION}

Lung cancer is a frequently occurring, mostly lethal disease in all countries worldwide. ${ }^{1}$ It is one of the most commonly diagnosed types of cancer and has the highest death rates ${ }^{2,3}$.

Lung cancers fall into two main categories: around $20 \%$ are small cell lung cancers (SCLC) and the remainder is non-small cell lung cancers (NSCLC). Depending on tumor size, location and histology, several treatment options are available, including surgery and radiotherapy (RT), both often combined with chemotherapy ${ }^{4}$. In limited disease SCLC concurrent chemoradiotherapy is the main treatment. For stage I and II operable NSCLC, surgical resection followed by adjuvant chemotherapy is the standard treatment in stage II, whereas the standard of care for inoperable stage I-II NSCLC is radiotherapy. Stage III NSCLC is treated with chemo-radiation, with surgery in selected cases ${ }^{5}$.

A major problem remains local tumor control ${ }^{6,7}$. Therefore, new ways to deliver radiotherapy beyond photons $s^{8,9}$ have been saught for, including protons ${ }^{8,9}$ and carbon ions ${ }^{10-12}$. Because of their superior dose-distribution, and for $\mathrm{C}$-ions also their biological characteristics, a therapeutic gain can be expected with charged particles ${ }^{13-}$ 24. Accordingly, particle therapy should help to minimize the extent and severity of pulmonary injury. This could benefit patients with localized lung cancer and severe underlying pulmonary disease, while simultaneously permitting an increased dose to the primary tumor, improving local tumor control. Chang et al reported that proton treatment significantly reduced dose to the esophagus, spinal cord, and heart, even with dose escalation, compared with photon therapy, either 3D or Intensity Modulated Radio Therapy (IMRT) ${ }^{25}$. Recently a number of studies systematically reviewed the effectiveness of radiotherapy with protons and carbon ions for a wide range of indications ${ }^{26-29}$. However, the present role of particle therapy in the treatment lung cancer is still unclear. This article therefore specifically aims to systematically review the currently available clinical evidence on particle therapy in lung cancer.

\section{METHODS}

\section{Search strategy and selection criteria}

The present review is based on a comprehensive systematic literature review by Lodge et al ${ }^{28}$, which was updated until November $1^{\text {st }} 2007$. The following electronic databases were used: CINAHL, EMBASE and MEDLINE. Search terms (using free text words as well as MESH terms) were used alone or in combination, related to lung cancer and charged particle treatment. This included the following terms: neoplasm, cancer, carcinoma, lung cancer, proton, ion, charged particle and hadron. 


\section{2 | Part 3: New Technologies- Chapter 7}

There was no limit applied by publication year, language or study design. Studies on animals only were excluded. After identifying search results, only studies in the English, French or German language which investigated protons and/or ions in the treatment of lung cancer with at least 20 patients and a follow up of 2 years, were included.

\section{Key outcomes and quality assessment}

Two independent reviewers assessed the trials, both for quality of the methods and for the results of key outcomes, which were identified and tabulated. Two reviewers (DDR, MPJ) extracted data independently to ensure validity, while a third reviewer $(P L)$ was responsible for resolving discrepancies.

We attempted to collect the following data from study reports: study design; particle type; initial disease stage; total tumor dose; fractionation; overall treatment time of the radiotherapy; local tumor control; survival; side effects (including the scoring system used); and quality of life assessment.

Detailed listing of patient-, treatment- and outcome aspects in the tables was performed for literature with:

- Patients' follow-up period > 24 months

- Publications which were entirely or partly based on the same patient data but address different issues the latest treatment results / largest patient population were further analyzed

\section{RESULTS}

\section{Search results}

Out of 5,089 search hits, 185 relevant papers regarding different types of cancer were identified as potentially relevant ${ }^{28}$. Of these 185 references, 134 duplicates and clearly irrelevant references were excluded. Through manual searches of the reference list, specialist journals, and correspondence with authors, no additional references were identified. On the remaining 51 studies we applied the above-mentioned restrictions, and selected only studies dealing with lung cancer. At the end, only 7 references were retrieved. An update of the review until November $1^{\text {st }} 2007$ yielded another 3 references, resulting in a total of 10 references.

\section{Description of the studies}

Ten studies were identified, all for NSCLC ${ }^{8-12,30-33}$. From these 10 studies, 5 studies $(n=214)$ investigated protons; 1 phase II study ${ }^{30}, 2$ prospective studies ${ }^{8,31}$ and 2 retrospective studies ${ }^{9,33}$. The remaining 5 studies, prospective and all performed at the same institution, investigated $C$-ions $(n=210)^{10-12,32,34}$. Study characteristics are tabulated in Table 1 and 2. 


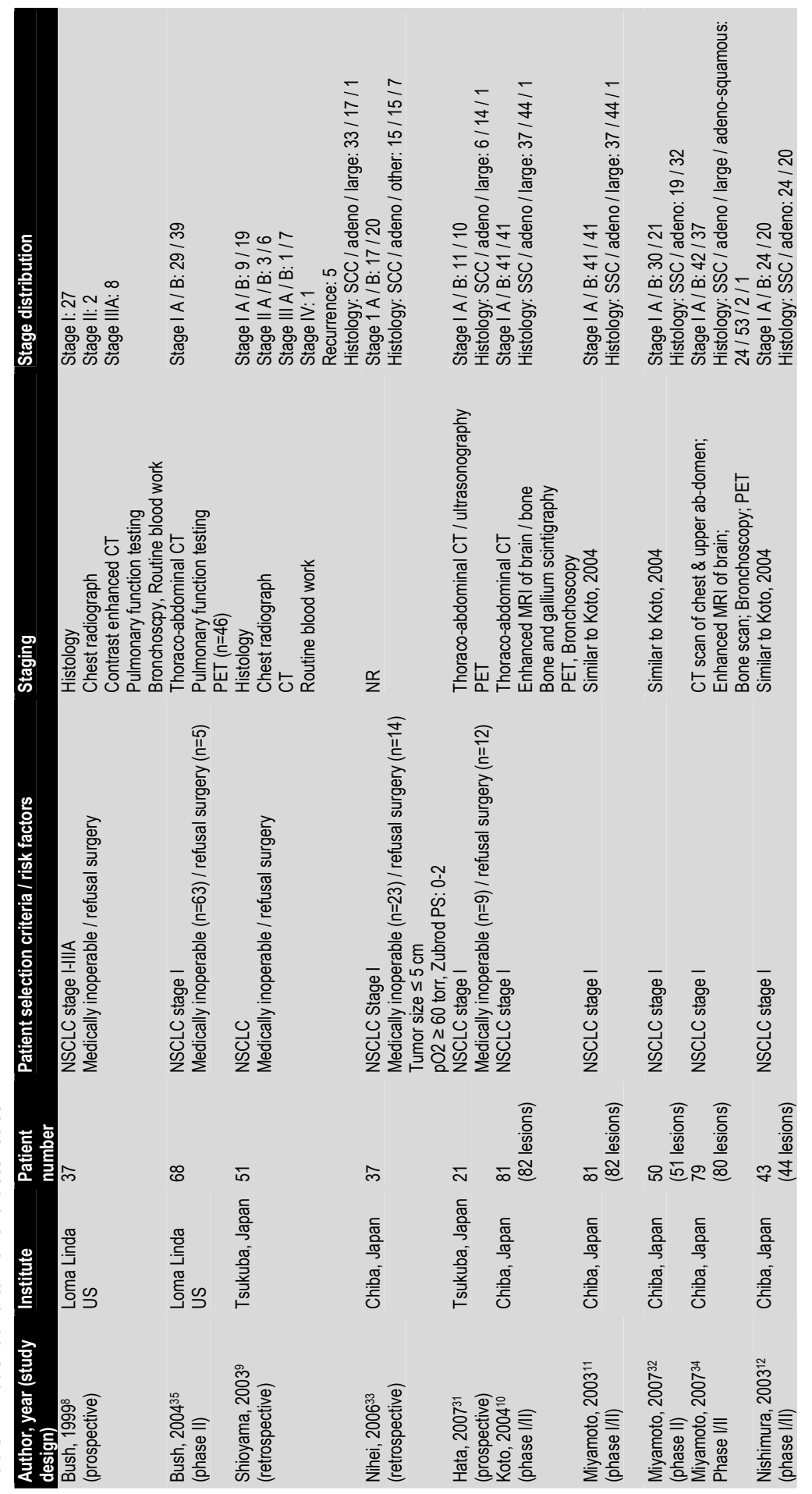




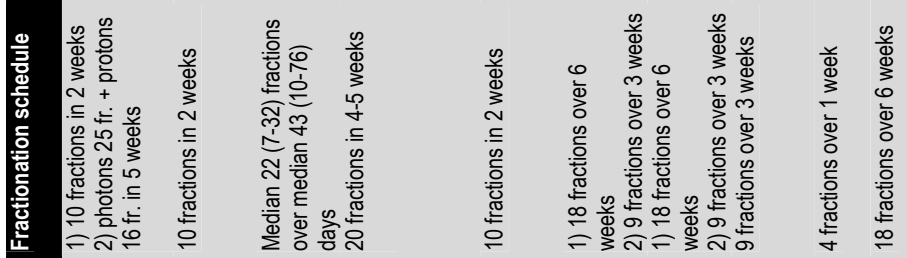

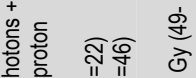
嵌交峲 至党

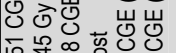

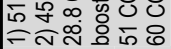

등 ত

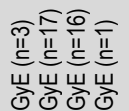
이용

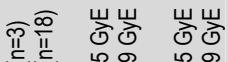

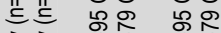

山 䓌志岕

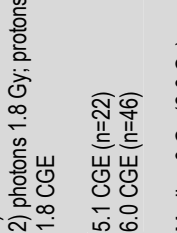

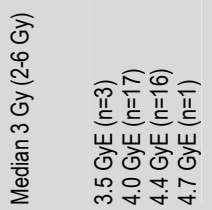

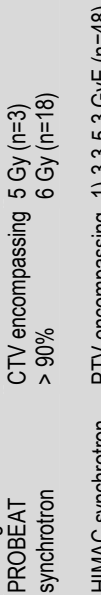

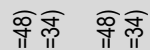

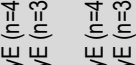

㐫岕 岕岕

mon

किं लं

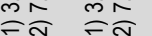

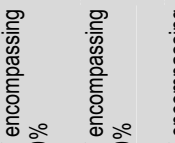

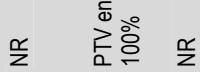

วоे ㅇํㅅ

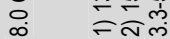

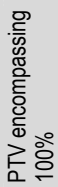

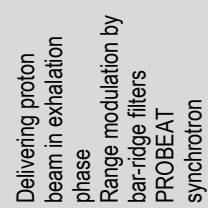

흔 흔

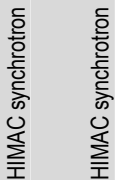

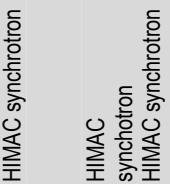

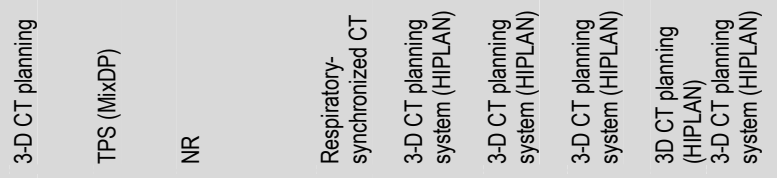

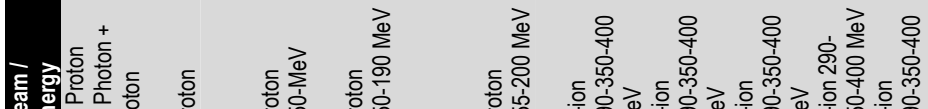
๓⿸户㔾

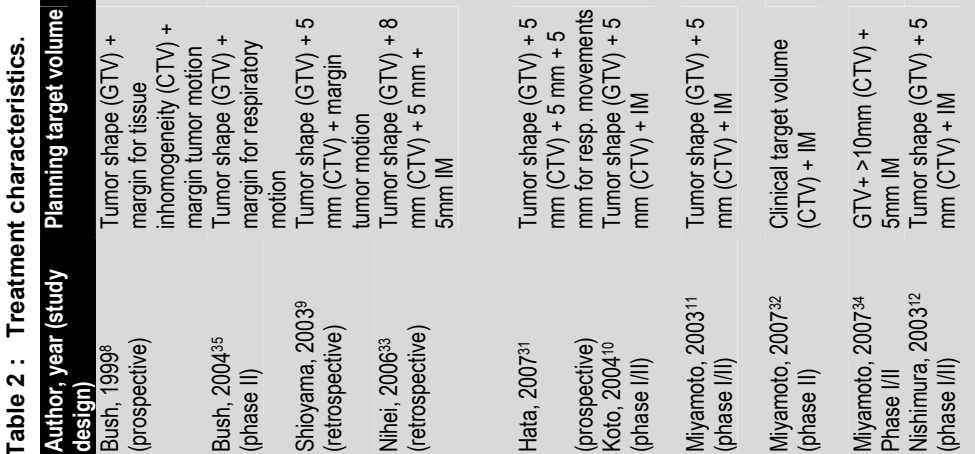


The prospective, non-randomized study of Bush et al. ${ }^{8}$ had two treatment arms. One arm was treated with protons only, while the other was treated with both photons and protons. Thirty-seven stage I-IIIA patients were assigned to one of the treatment arms according to pulmonary reserve and cardiac function. Patients in the first arm of the study were given proton beam radiation therapy that covered only the gross tumor volume as identified on the planning CT scan. These patients received 10 daily fractions of 5.1 cobalt Gray equivalents (CGE) for a total of 51 CGE in 2 weeks. The second arm of the study included patients judged suitable for elective mediastinal irradiation (45 Gy), based on their lung function. Such patients had a $F E V_{1}$ of $>1 \mathrm{~L}$ and no evidence of severe cardiac insufficiency. Concomitant with the last 16 photon treatments, a proton boost to the primary tumor was given with a total tumor dose of 73.8 CGE in 5 weeks.

The phase II study of Bush et al. ${ }^{35}$ included only patients with stage I disease $(n=68)$. They were given 10 proton fractions in 2 weeks, with a total dose of 51 CGE for the first 22 patients and 60 CGE for the later 46 patients.

One retrospective study from Japan ${ }^{9}$ evaluated 51 stage I-IV patients. Fractionation regimens for proton therapy varied as a result of irregularly allocated beam time. The median fraction size and total doses were 3.0 Gy (range 2-6 Gy) and 76 Gy (range 49-93 Gy), respectively. The median overall treatment time was 43 days (range 10-76 days).

The other retrospective study, also from Japan, evaluated 37 stage I patients $^{33}$. The tumor size of all patients was $\leq 5 \mathrm{~cm}$ in diameter and all had a Zubrod performance status $0-2$. Ten of those 37 patients were enrolled in an earlier performed phase I dose escalation study (70-90 CGE in 20 fractions) in the same institution. The remaining 27 patients were treated with either a dose of 88 CGE (4.4CGE/20fr) ( $n=13)$ or with a dose of 80 CGE (4CGE/20fr) $(n=14)$.

A recent prospective study from Japan ${ }^{31}$ investigated 21 stage I patients. The first three patients were treated with a total dose of $50 \mathrm{~Gy}$ in 10 fractions over 2 weeks. When no therapy-related toxicities of grade 3 or higher were observed during three months, the total dose was escalated to $60 \mathrm{~Gy}$, also in 10 fractions over 2 weeks. Survival, local progression-free and disease-free rates were calculated according to the Kaplan-Meier method.

Miyamoto et al. ${ }^{11}$ conducted a prospective phase $\mathrm{I} / \mathrm{Il}$ dose-escalation study with $\mathrm{C}$ ions in stage I NSCLC patients, which was carried out by two different protocols. The first stage phase I/II trial used 18 fractions over 6 weeks in 47 patients and the second one delivered nine fractions over 3 weeks in 34 patients. A dose escalation from 59.4 to 95.4 CGE in incremental steps of $10 \%$ and from 68.4 to 79.2 CGE in $5 \%$ increments was done in the two stages of the trial, respectively. The local control and survival rates were obtained using the Kaplan-Meier method.

Recently the same investigator supdated their results of 50 patients in a phase II trial $^{32}$, receiving 9 fractions of 8 CGE over 3 weeks. Subsequently 79 patients were treated in a phase I/II trial, receiving 4 fractions of 13.2 CGE (stage IA) or 15 CGE 
(stage IB) over 1 week $^{34}$. Survival, local progression-free and disease-free rates were also calculated according to the Kaplan-Meier method.

Koto et al. ${ }^{10}$ used the same study population as described above by the study of Miyamoto but investigated in identifying the incidence of in-field recurrences by collating the dose distribution with the CT images.

The same study population was also used in a study of Nishumura ${ }^{12}$. We included this study because this report focused on the pulmonary side effects of C-ion therapy, which were not mentioned in the previous studies.

\section{Local tumor control}

With regard to protons, in the first published prospective study ${ }^{8}$, the 2 -year local tumor control rate was $87 \%$ (Table 3 ). The phase II study of Bush et $\mathrm{al}^{35}$, with an overall treatment time (OTT) of 2 weeks, reported a 3-year local tumor control of $74 \%$, which differed between stage IA (87\%) and stage IB (49\%) tumors. In the retrospective study of Shioyama et al. ${ }^{9}$, a 5 -year local tumor control rate of $57 \%$ for stage I NSCLC patients $(n=28)$ was reported. The local tumor control rate of the remaining patients with stage II-IV in this study was unclear. Nihei and his colleagues reported in another retrospective study a locoregional relapse free survival rate of $79 \%$ in stage IA of $60 \%$ in stage $\mathrm{IB}^{33}$. Hata et $\mathrm{al}^{31}$, also with an OTT of 2 weeks, found a local progression free rate at 2 years of $95 \%$.

The local tumor control rate in the Miyamoto study on C-ions ${ }^{11}$, that only included stage I patients, was $74 \%$. Koto et $\mathrm{al}^{10}$ concluded that the local control was dose-dependent and determined the optical therapeutic dose based on their findings. In their update Miyomato and colleagues ${ }^{32}(n=50)$ reported a 5 -year local control of $95 \%$. The results of the recently published phase $\mathrm{I} / \mathrm{II}$ trial of the same investigators showed a 5 -year local control rate of $90 \%$ (total group), $98 \%$ (stage $1 \mathrm{~A}$ ) and $80 \%(\text { stage } 1 \mathrm{~B})^{34}$.

\section{Survival}

Bush et al. ${ }^{8}$ reported actuarial overall survival after proton therapy at 2 years for the entire group of $31 \%$, and for clinical stage I patients of $39 \%$. The disease-free survival rate at 2 years was $63 \%$ for the entire group, $85 \%$ for stage I patients and $19 \%$ for stage IIIA patients. The phase II study of Bush et al. ${ }^{35}$ reported overall survival at 3 years of $44 \%$, depending on whether a total dose of 51 CGE $(27 \%)$ or 60 CGE $(55 \%)$ was given. Disease-specific survival rate at 3 years was $72 \%$.

In the retrospective study of Nihei et al, ${ }^{33}$, the reported 2 year local progression-free and overall survival rates were $80 \%$ and $84 \%$ respectively. Shioyama et al. ${ }^{9}$ reported, for all patients, 5-year overall survival and disease-free survival rates of $29 \%$ and $37 \%$ respectively. The 5 -year cause-specific survival rate was $46 \%$ for 37 stage I-II patients and a 2-year cause-specific survival rate of $70 \%$ for 9 stage III-IV patients. Hata et $\mathrm{al}^{31}$ reported a 2 -year overall and cause-specific survival rate of $74 \%$ and $86 \%$ respectively. 

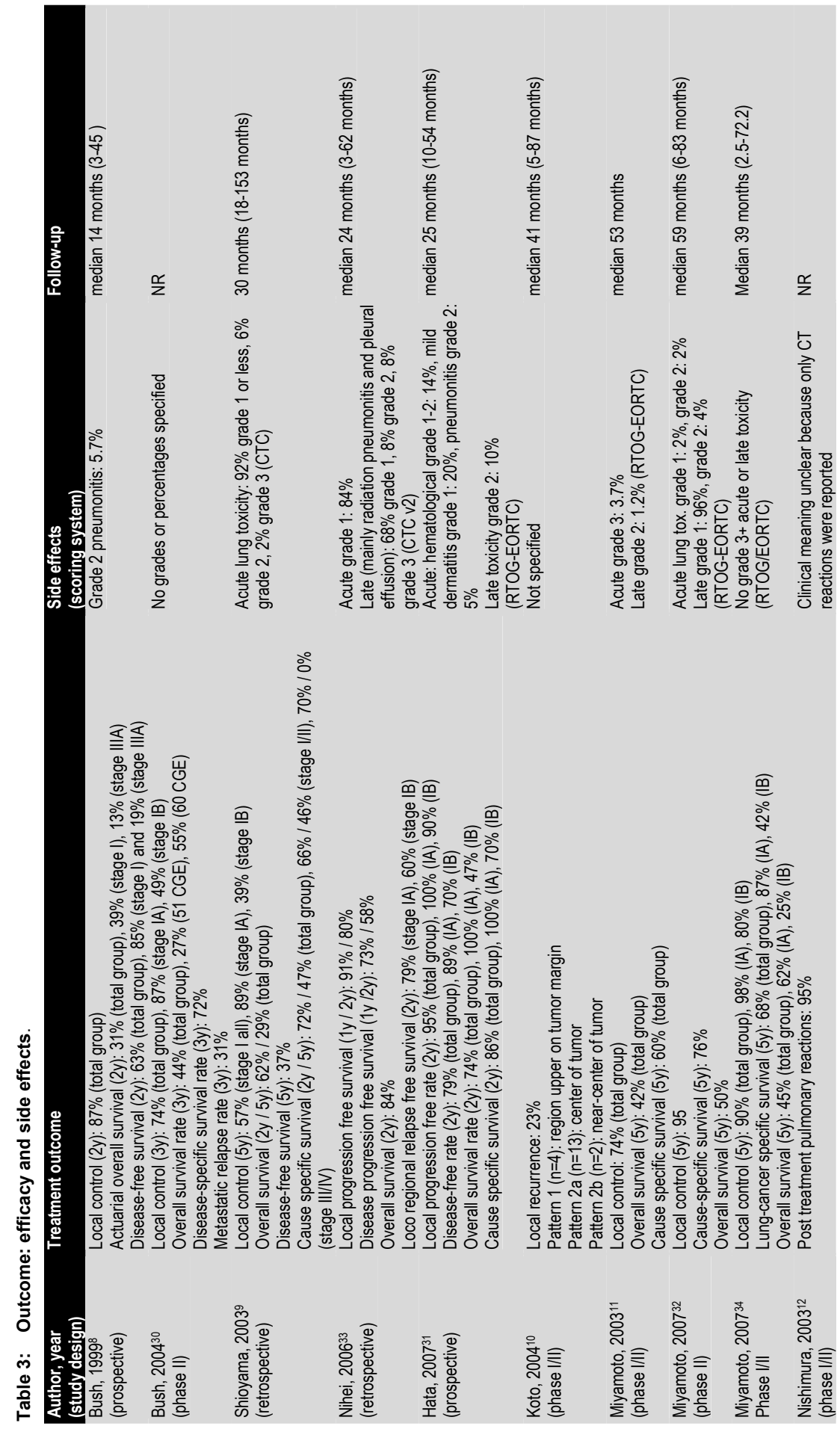
The study of Koto et $\mathrm{al}^{10}$ on $\mathrm{C}$-ions, with a median follow-up period of 40.6 months (range 4.9-86.9 months), reported a survival rate of $45.7 \%$ for the entire group, all stage I. The rates for 5 -year overall and cause-specific survival in the study with the same study population of Miyamoto et al. (2003) were in 81 stage I patients $42 \%$ and $60 \%$, respectively. The 50 patients in the phase II study of Miyamoto et al ${ }^{32}$ had overall and cause-specific survival rates of $50 \%$ and $76 \%$ respectively. In the recently phase I/II trial of the same investigators ${ }^{34}$ the reported 5 year lung-cancer specific survival was $68 \%$ (total group), $87 \%$ (stage IA) and $42 \%$ (stage IB), whereas the 5 year overall survival was $45 \%$ (total group), $62 \%$ (stage IA) and $25 \%$ (stage IB).

\section{Side effects and QoL}

In the prospective proton study of Bush et al ${ }^{8}, 5.7 \%$ of the patients developed a grade 2 pneumonitis (scoring system was unclear). In the retrospective study of Shioyama et al. ${ }^{9}$, only 1 patient $(2 \%)$ developed a grade 3 toxicity according to the CTC scoring system. In the study of Hata et $\mathrm{al}^{31}$ toxicity was scored using the RTOG/EORTC system. Of the patients $14 \%$ developed acute hematological toxicity of grade $1-2,20 \%$ developed acute mild dermatitis grade 1 and 1 patient (5\%) developed acute radiation pneumonitis grade 2 . Late toxicity of grade 2 was developed by $10 \%$ of the patients.

With regard to the C-ion studies, Nishimura et al. ${ }^{12}$ reported only CT reactions, with use of the LENT/SOMA scorings system. Miyamoto ${ }^{11}$ reported in $3.7 \%$ of the patients acute side effects (grade 3 and more) and 1.2\% late side effects (grade 3 and more), according to the RTOG/EORTC system. In their update Miyamoto et $\mathrm{al}^{32}$ reported acute lung toxicity grade 1 or 2 in $4 \%$ of the patients, and late lung toxicity grade 2 also in $4 \%$ of the patients. In the recently published phase $1 / I$ trial of the same investigators where a total dose of 52.8-60 CGE was delivered over 1 week, no grade3+ acute or late toxicity was observed ${ }^{34}$.

Quality of life was reported in none of the above-mentioned studies.

\section{DISCUSSION}

Local tumor control is still low for lung cancer, both for early and advanced disease 6,7 . Because of its theoretical advantages, both protons and light ions show great promise for they may enable lowering the dose to the normal tissues, as well as allow radiation dose escalation and hence increased tumor control ${ }^{36}$. On top of that, light ions, having a higher biological effectiveness than protons or photons, may further increase the effectiveness of charged particles beyond that of protons ${ }^{37}$.

In this review, we searched for clinical evidence that protons or light ions would benefit patients with lung cancer. Using a systematic approach only 10 fully published 
series, all dealing with NSCLC, were identified ${ }^{8-12,31-35}$. Studies on proton therapy were performed in Japan or the United States, while for studies on C-ions we were limited to the experience in Japan. In total, only 424 patients were treated with protons or $\mathrm{C}$-ions, all in retrospective or prospective phase I/II trials. No phase III trials could be identified. Only 33 patients had stage II or III disease, all others had stage I. In $46-95 \%$ of the patients local tumor control was achieved and less than $8 \%$ of the patients experienced severe late side effects (grade $3+$ ) afer proton treatment, whereas after $\mathrm{C}$-ion treatment no late grade $3+$ side effects were observed.

It is rather difficult to compare the outcomes of different types of radiation in different studies, as different equipment, planning systems, doses, fractions and fractionation schedules are used. Nevertheless, the results, at least for stage I NSCLC, seem to be better than what is generally achieved by conventional radiotherapy ${ }^{38-40}$. However, the results presented in this paper seem to be inferior to what may be achieved with hypofractionated "stereotactic" photon techniques, with which local tumor control rates of $90 \%$ or more were reported ${ }^{41-44}$. In general, the local control rates for proton therapy of $87 \%$ (2-year) $)^{8}, 74 \%$ (3-year) ${ }^{30}$ and $57 \%\left(5\right.$-year) ${ }^{9}$ are not higher than those achieved with stereotactic radiotherapy with photons ${ }^{41,42} 43,44,46$. Nevertheless, the local progression free rate of $95 \%$ that was found by Hata et $\mathrm{al}^{31}$ is equivalent to the results of Timmerman et al with photons ${ }^{45}$. The cause specific survival (CSS) and overall survival (OS) rates achieved with proton therapy are equivalent or even inferior to treatment with photons ${ }^{42,43,46}$, with weighted means for $2 y / 5 y$ CCS and $2 y / 5 y$ OS of $80 \% / 72 \%$ and $59 / 44 \%$, respectively $(\text { Table } 4)^{28}$. The better proton results of Hata et $\mathrm{al}^{31}$ compared to the other proton series described in this paper, could be explained by an increased Biological Effective Doses (BED) due to the hypofractionated radiation schedule with high fraction doses (5-6 CGE) and a short OTT (2 weeks). This is in contrast to the early phase of particle treatment, where too low BED to obtain local tumor control have been used. An equal radiation schedule was used by Bush et $\mathrm{al}^{35}$. The results of the 2 year cause specific survival of this latter study were also better as compared to other proton series ( $72 \%$ vs. $58-66 \%)$. This result is in line with earlier publications on NSCLC, were advantages by using schedules with a short OTT have been confirmed ${ }^{47,48}$.

Treatment with C-ions, especially according to the recent study of Miyamoto et $\mathrm{al}^{32}$, seems also to result very high local tumor control rates (5y LC=95\%). Their 5-year overall survival is somewhat higher than that of photon studies ${ }^{43}(50 \%$ vs. $30 \%$ and $47 \%$ respectively), and 5-year cause-specific survival is comparable to the best achievements in photon therapy ${ }^{46}(76 \%$ vs. $78 \%)$. However, as these C-ion outcomes are found in only one study with 50 patients, they should be interpreted with caution.

In all of the included studies, the incidence of reported severe side effects was relatively low. Taking the most recent publications (2007) into account the percentage late toxicity was similar in the group of patients receiving proton therapy, and even 


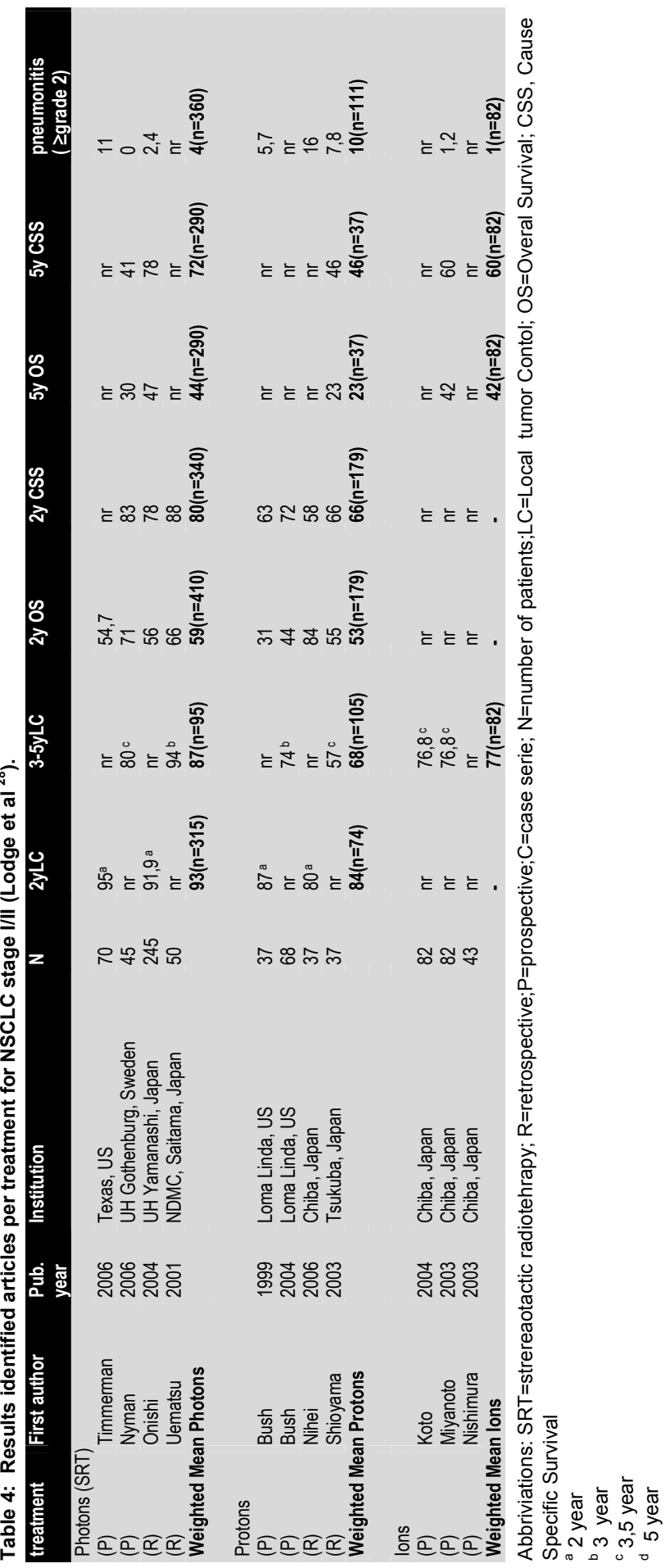


lower after treatment with C-ions, as compared to SRT; proton $^{31}: 10 \%$ (grade 2), Cion $^{32,34}$ : 4\% (grade 2) and SRT ${ }^{45,46,49}$ : $9 \%$ (grade 2-5, range: $5 \%-20 \%$ ). Due to the relatively low number of patients, the results regarding late severe toxicity after particle therapy should be interpreted with caution. We could not draw any conclusions about QoL, since in none of the included studies QoL was reported.

Because of the overwhelming theoretical data on the beneficial properties of protons and light ions ${ }^{13-18,20-23}$, as well as some promising recent results, further investment in the infrastructure needed to perform large trials in patients with different lung cancer stages, is warranted. Also, studies should investigate the possible role of $\mathrm{C}$-ion therapy in SCLC, as also in this histology, local tumor failure occurs in at least $30 \%$ of patients ${ }^{50}$. Nakano and collegues ${ }^{51}$ showed in their study on bulky cervical cancer (stage IIIb and IVa) that the intratumoral $\mathrm{pO}_{2}$ status during C-ion therapy had no influence on either disease-free survival or local control probability. Hence, it would be worthwhile to investigate in clinical studies with hypoxic tumors like SCLC or advanced NSCLC.

In the meantime, the complexity introduced by tumor motion must be recognized ${ }^{52-55}$. However, as usually the particle beam treats the whole target volume, the chance of missing the target will reduce as compared with IMRT were only a portion of the target is treated per beam.

Available data demonstrate that particle therapy in general and specifically proton therapy is a safe and feasible treatment modality, thus having successfully past the phase I stage of an experimental therapy. However, due to a lack of direct comparisons between particle therapy and the best available photon treatment, the clinical efficacy of most indications remains unclear, resulting also in unproven costeffectiveness. Since particle therapy is a relatively expensive treatment modality, the latter is of major importance and can be considered as the main stagnation factor in the start-up phase of new particle centers. Nevertheless, at current operating centers, particle therapy is already implemented as daily practice for several indications (skull base chordomas, chondrosarcomas, pediatric tumors, ocular melanomas, adenoid cystic carcinomas). Even though it is doubtful whether randomized clinical trials are possible and ethical for protons ${ }^{56}$, we recommend this item to be brought to the forefront of discussions in international radiotherapy working groups, in particular with professional associations like ASTRO and ESTRO in order to establish international cooperation on clinical research in the field of particle therapy.

Although current results are promising, in this era of evidence-based medicine, more evidence is required before particle therapy can become internationally the standard treatment for (subsets of) lung cancer patients. 


\section{2 | Part 3: New Technologies- Chapter 7}

\section{REFERENCES}

1. Shibuya K, Mathers CD, Boschi-Pinto C, et al: Global and regional estimates of cancer mortality and incidence by site: II. Results for the global burden of disease 2000. BMC Cancer 2:37, 2002

2. Jemal A, Siegel R, Ward E, et al: Cancer statistics, 2007. CA Cancer J Clin 57:43-66, 2007

3. Ferlay J, Autier P, Boniol M, et al: Estimates of the cancer incidence and mortality in Europe in 2006. Annals of oncology 18:581-92, 2007

4. Jassem J: The role of radiotherapy in lung cancer: where is the evidence? Radiother Oncol 83:203-13, 2007

5. SIGN: Management of patients with lung cancer: A national clinical guidline, in NHS (ed): Quality Improvement Scotland, 2005

6. Kim YS, Yoon SM, Choi EK, et al: Phase II study of radiotherapy with three-dimensional conformal boost concurrent with paclitaxel and cisplatin for Stage IIIB non-small-cell lung cancer. Int J Radiat Oncol Biol Phys 62:76-81, 2005

7. Farray D, Mirkovic N, Albain KS: Multimodality therapy for stage III non-small-cell lung cancer. J Clin Oncol 23:3257-69, 2005

8. Bush DA, Slater JD, Bonnet R, et al: Proton-beam radiotherapy for early-stage lung cancer. Chest 116:1313-9, 1999

9. Shioyama $\mathrm{Y}$, Tokuuye $\mathrm{K}$, Okumura $\mathrm{T}$, et al: Clinical evaluation of proton radiotherapy for non-small-cell lung cancer. Int J Radiat Oncol Biol Phys 56:7-13, 2003

10. Koto M, Miyamoto $\mathrm{T}$, Yamamoto $\mathrm{N}$, et al: Local control and recurrence of stage I non-small cell lung cancer after carbon ion radiotherapy. Radiother Oncol 71:147-56, 2004

11. Miyamoto $\mathrm{T}$, Yamamoto $\mathrm{N}$, Nishimura $\mathrm{H}$, et al: Carbon ion radiotherapy for stage I non-small cell lung cancer. Radiother Oncol 66:127-40, 2003

12. Nishimura H, Miyamoto $\mathrm{T}$, Yamamoto $\mathrm{N}$, et al: Radiographic pulmonary and pleural changes after carbon ion irradiation. Int J Radiat Oncol Biol Phys 55:861-6, 2003

13. Krengli M, Hug EB, Adams JA, et al: Proton radiation therapy for retinoblastoma: comparison of various intraocular tumor locations and beam arrangements. Int J Radiat Oncol Biol Phys 61:583-93, 2005

14. St Clair WH, Adams JA, Bues M, et al: Advantage of protons compared to conventional X-ray or IMRT in the treatment of a pediatric patient with medulloblastoma. Int J Radiat Oncol Biol Phys 58:727-34, 2004

15. Weber DC, Trofimov AV, Delaney TF, et al: A treatment planning comparison of intensity modulated photon and proton therapy for paraspinal sarcomas. Int J Radiat Oncol Biol Phys 58:1596-606, 2004

16. Baumert BG, Lomax AJ, Miltchev $\mathrm{V}$, et al: A comparison of dose distributions of proton and photon beams in stereotactic conformal radiotherapy of brain lesions. Int J Radiat Oncol Biol Phys 49:1439-49, 2001

17. Zurlo A, Lomax A, Hoess A, et al: The role of proton therapy in the treatment of large irradiation volumes: a comparative planning study of pancreatic and biliary tumors. Int $\mathrm{J}$ Radiat Oncol Biol Phys 48:277-88, 2000

18. Miralbell R, Crowell C, Suit HD: Potential improvement of three dimension treatment planning and proton therapy in the outcome of maxillary sinus cancer. Int J Radiat Oncol Biol Phys 22:305-10, 1992

19. Miralbell R, Lomax A, Russo M: Potential role of proton therapy in the treatment of pediatric medulloblastoma/primitive neuro-ectodermal tumors: spinal theca irradiation. Int $\mathrm{J}$ Radiat Oncol Biol Phys 38:805-11, 1997

20. Cozzi L, Fogliata A, Lomax A, et al: A treatment planning comparison of 3D conformal therapy, intensity modulated photon therapy and proton therapy for treatment of advanced head and neck tumours. Radiother Oncol 61:287-97, 2001

21. Lee $\mathrm{CH}$, Tait $\mathrm{D}$, Nahum $\mathrm{AE}$, et al: Comparison of proton therapy and conformal X-ray therapy in nonsmall cell lung cancer (NSCLC). Br J Radiol 72:1078-84, 1999 
22. Fogliata A, Bolsi A, Cozzi L: Critical appraisal of treatment techniques based on conventional photon beams, intensity modulated photon beams and proton beams for therapy of intact breast. Radiother Oncol 62:137-45, 2002

23. Isacsson $U$, Montelius A, Jung B, et al: Comparative treatment planning between proton and X-ray herapy in locally advanced rectal cancer. Radiother Oncol 41:263-72, 1996

24. Isacsson $\mathrm{U}$, Hagberg $\mathrm{H}$, Johansson $\mathrm{KA}$, et al: Potential advantages of protons over conventional radiation beams for paraspinal tumours. Radiother Oncol 45:63-70, 1997

25. Chang JY, Zhang X, Wang X, et al: Significant reduction of normal tissue dose by proton radiotherapy compared with three-dimensional conformal or intensity-modulated radiation therapy in Stage I or Stage III non-small-cell lung cancer. International journal of radiation oncology, biology, physics 65:1087-96, 2006

26. Brada M, Pijls-Johannesma M, De Ruysscher D: Proton therapy in clinical practice: current clinical evidence. Journal of clinical oncology 25:965-70, 2007

27. Greco C, Wolden S: Current status of radiotherapy with proton and light ion beams. Cancer 109:122738, 2007

28. Lodge M, Pijls-Johannesma M, Stirk L, et al: A systematic literature review of the clinical and costeffectiveness of hadron therapy in cancer>. Radiother Oncol 83:110-22, 2007

29. Olsen DR, Bruland OS, Frykholm G, et al: Proton therapy - A systematic review of clinical effectiveness. Radiother Oncol 83:123-32, 2007

30. Bush DA, Slater JD, Shin BB, et al: Hypofractionated proton beam radiotherapy for stage I lung cancer. Chest 126:1198-203, 2004

31. Hata M, Tokuuye K, Kagei K, et al: Hypofractionated high-dose proton beam therapy for stage I nonsmall-cell lung cancer: preliminary results of a phase I/II clinical study. Int $\mathrm{J}$ Radiat Oncol Biol Phys 68:786-93, 2007

32. Miyamoto T, Baba M, Yamamoto N, et al: Curative treatment of Stage I non-small-cell lung cancer with carbon ion beams using a hypofractionated regimen. Int J Radiat Oncol Biol Phys 67:750-8, 2007

33. Nihei K, Ogino T, Ishikura S, et al: High-dose proton beam therapy for Stage I non-small-cell lung cancer. Int J Radiat Oncol Biol Phys 65:107-11, 2006

34. Miyamoto T, Baba M, Sugane $\mathrm{T}$, et al: Carbon ion radiotherapy for stage I non-small cell lung cancer using a regimen of four fractions during 1 week. J Thorac Oncol 2:916-26, 2007

35. Bush DA, Hillebrand DJ, Slater JM, et al: High-dose proton beam radiotherapy of hepatocellular carcinoma: preliminary results of a phase II trial. Gastroenterology 127:S189-93, 2004

36. Chang JY, Liu HH, Komaki R: Intensity modulated radiation therapy and proton radiotherapy for nonsmall cell lung cancer. Curr Oncol Rep 7:255-9, 2005

37. Orecchia R, Zurlo A, Loasses A, et al: Particle beam therapy (hadrontherapy): basis for interest and clinical experience. Eur J Cancer 34:459-68, 1998

38. Sirzen F, Kjellen E, Sorenson S, et al: A systematic overview of radiation therapy effects in non-small cell lung cancer. Acta Oncol 42:493-515, 2003

39. Jeremic B, Classen J, Bamberg M: Radiotherapy alone in technically operable, medically inoperable, early-stage (I/II) non-small-cell lung cancer. Int J Radiat Oncol Biol Phys 54:119-130, 2002

40. Qiao X, Tullgren O, Lax I, et al: The role of radiotherapy in treatment of stage I non-small cell lung cancer. Lung Cancer 41:1-11, 2003

41. Timmerman R, Papiez L, McGarry R, et al: Extracranial stereotactic radioablation: results of a phase I study in medically inoperable stage I non-small cell lung cancer. Chest 124:1946-55, 2003

42. Uematsu M, Shioda A, Suda A, et al: Computed tomography-guided frameless stereotactic radiotherapy for stage I non-small cell lung cancer: a 5-year experience. Int J Radiat Oncol Biol Phys 51:666-70, 2001

43. Nyman J, Johansson KA, Hulten U: Stereotactic hypofractionated radiotherapy for stage I non-small cell lung cancer--mature results for medically inoperable patients. Lung Cancer 51:97-103, 2006 


\section{4 | Part 3: New Technologies- Chapter 7}

44. Onishi H, Araki T, Shirato $\mathrm{H}$, et al: Stereotactic hypofractionated high-dose irradiation for stage I nonsmall cell lung carcinoma: clinical outcomes in 245 subjects in a Japanese multiinstitutional study. Cancer 101:1623-31, 2004

45. Timmerman R, McGarry R, Yiannoutsos C, et al: Excessive toxicity when treating central tumors in a phase II study of stereotactic body radiation therapy for medically inoperable early-stage lung cancer. J Clin Oncol 24:4833-9, 2006

46. Onishi H, Shirato H, Nagata $\mathrm{Y}$, et al: Hypofractionated stereotactic radiotherapy (HypoFXSRT) for stage I non-small cell lung cancer: updated results of 257 patients in a Japanese multi-institutional study. J Thorac Oncol 2:S94-100, 2007

47. Fowler JF, Tome WA, Fenwick JD, et al: A challenge to traditional radiation oncology. Int $\mathrm{J}$ Radiat Oncol Biol Phys 60:1241-56, 2004

48. Saunders $M$, Dische S, Barrett $A$, et al: Continuous, hyperfractionated, accelerated radiotherapy (CHART) versus conventional radiotherapy in non-small cell lung cancer: mature data from the randomised multicentre trial. CHART Steering committee. Radiother Oncol 52:137-48, 1999

49. Zimmermann FB, Geinitz H, Schill S, et al: Stereotactic hypofractionated radiotherapy in stage I (T1-2 NO M0) non-small-cell lung cancer (NSCLC). Acta Oncol 45:796-801, 2006

50. Turrisi AT, 3rd, Kim K, Blum R, et al: Twice-daily compared with once-daily thoracic radiotherapy in limited small-cell lung cancer treated concurrently with cisplatin and etoposide. N Engl J Med 340:26571,1999

51. Nakano T, Suzuki Y, Ohno T, et al: Carbon beam therapy overcomes the radiation resistance of uterine cervical cancer originating from hypoxia. Clin Cancer Res 12:2185-90, 2006

52. Bush DA, Slater JD, Garberoglio $C$, et al: A technique of partial breast irradiation utilizing proton beam radiotherapy: comparison with conformal x-ray therapy. Cancer J 13:114-8, 2007

53. Engelsman M, Rietzel E, Kooy HM: Four-dimensional proton treatment planning for lung tumors. Int $\mathrm{J}$ Radiat Oncol Biol Phys 64:1589-95, 2006

54. Kang $\mathrm{Y}$, Zhang X, Chang JY, et al: 4D Proton treatment planning strategy for mobile lung tumors. Int J Radiat Oncol Biol Phys 67:906-14, 2007

55. Mori S, Endo M, Komatsu S, et al: Four-dimensional measurement of lung tumor displacement using 256-multi-slice CT-scanner. Lung Cancer 56:59-67, 2007

56. Glimelius B, Montelius A: Proton beam therapy - Do we need the randomised trials and can we do them? Radiother Oncol 83:105-9, 2007 


\section{CHAPTER}

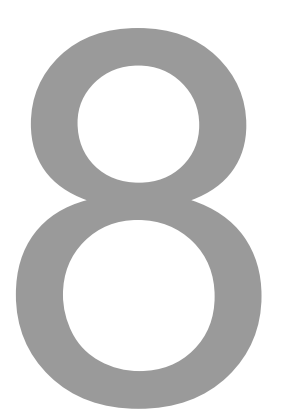

\section{Cost-effectiveness of particle therapy: current evidence and future needs}

Radiotherapy \& Oncology (2008), in press

Madelon Pijls-Johannesma

Pascal Pommier Yolande Lievens 


\title{
126 | Part 3: New technologies- Chapter 8
}

\begin{abstract}
Purpose: Questions are being raised regarding the cost of particle therapy (PT), and with them criticism that PT is too expensive to allow the expected gain in effectiveness. This paper aims to get more insight in the cost and cost-effectiveness of particle therapy and to discuss a future strategy that allows for critical assessment of this health technology.
\end{abstract}

Material and methods: A systematic literature review based on an earlier published comprehensive review was performed and updated until June $1^{\text {st }} 2008$. Besides current business plans of PT projects were examined. Additionally, results retrieved from a cost-simulation tool developed under auspice of the ENLIGHT were discussed.

Results: The current literature on cost-effectiveness of PT is scarce, noncomparable, and largely not performed according to standard health technology assessment criteria. Besides, different perspectives for cost evaluations have been used, making it difficult to compare and to determine the relative impact in terms of costs for this new treatment modality.

Conclusion: Evidence on the cost-effectiveness of PT is scarce. Adequate reimbursement is necessary to support such innovative yet costly treatments. For now, model-based economic evaluations performed at least from a health care perspective may help us to gain evidence-based insight into cost-effectiveness. 


\section{INTRODUCTION}

Despite the potential physical and biological advantages of particle therapy (PT) as shown in many planning studies (tabulated in Glimelius et $\mathrm{al}^{1}$ ), there is little current evidence on the effectiveness of radiotherapy with protons or carbon-ions ${ }^{2-4}$. However, due to improvements in equipment and techniques as well a common interest to design international prospective comparison trials, this evidence may start to come to light. Meanwhile, various centers have been established and questions raised regarding the cost of particle therapy treatments, bringing with them the risk of criticism that these treatments are too expensive to allow the expected gain in effectiveness. Ideally, the total (investment as well as operational) costs incurred by radiation treatment should be covered by adequate reimbursement ${ }^{5}$. Without this positive financial incentive, departments and institutes are unlikely to be eager to deliver or implement new techniques. In order to obtain reimbursement for costly treatments in the face of governmental pressure to limit the (escalation in) health care expenses, new techniques must prove that additional higher cost are worhtwile, thus cost-effective.

This puts the introduction of new irradiation treatments such as particle therapy in a difficult situation: firm data on effectiveness can only be obtained after the implementation and clinical evaluation of the treatment, which in the case of radiotherapy often implies that important capital investments must be made. Yet data on effectiveness will only become available years after the necessary investment in equipment, buildings and highly qualified personnel. As mentioned above, effectiveness data on PT treatments are lacking; regardless, sufficient financing is necessary to perform clinical research and ensure the eventual dissemination of evidence-based treatments into clinical practice.

A number of questions have recently been raised and served as the subject of two editorials ${ }^{6,7}$. First, do we really need empirical evidence to determine effectiveness? Is it ethical to randomize patients between two treatments when it is obvious from a theoretical point of view that one is better than the other? What kind of trial needs to be designed to answer the question of whether a clinical improvement is worth the added expense? Moreover, even if a prospective clinical trial would be feasible - be it a randomized clinical trial or a matched-case study - it would take at least several years to obtain the results. Yet, for political and clinical decision-making, there is a current need for answers to these crucial questions.

Furthermore, at current, there is an ongoing discussion about the requirement whether to perform randomized trials or not before implementing new technologies ${ }^{8-}$ ${ }^{10}$. All three latter publications indicated that it would be unethical to randomize patients between protons and photons based on violence of the principles of equipoise between both treatment arms. Suit et $\mathrm{al}^{10}$ stated that if proton therapy were less expensive than X-ray therapy, there would be no interest in conducting phase III trials. 
Therefore, the aim of this article is to get more insight in the cost and costeffectiveness of particle therapy and to discuss a future strategy that allows for critical assessment of this health technology as was recommended by Bentzen ${ }^{8}$.

\section{MATERIAL AND METHODS}

An important issue which is gaining increasing importance in the field of radiation oncology is how the costs of treatments relate to their effects. Typically, the extra cost of a new intervention (compared to the standard treatment) must be balanced with its additional gain in clinical effect (i.e., better local control and/or survival, decreased morbidity) by means of economic evaluations. Some basics about economic evaluations are reviewed below.

There are four types of economic evaluations: 1. Cost-benefit analysis (CBA); 2. Cost-utility analysis (CUA); 3. Cost-effectiveness analysis (CEA); and 4. Costminimization analysis (CMA). The differences between the four economic evaluations are depicted in Table 2. From Table 1 it will be clear that only evaluations investigating both costs and effects, with a comparison between alternatives, can claim to provide full economic evaluation.

For an evaluation of new treatment modalities, it is mostly long-term data that are required to estimate therapy effects. Such data are, however, difficult to obtain. Clinical trials mostly have a limited follow-up. One method which allows the meaningful combination of data from different data sets as well as extrapolation is known as modeling. $^{11}$

Models allow real-life situations to be represented in a mathematical or a statistical manner. Mathematical modeling is used widely in the economic evaluation of pharmaceutical and healthcare technologies.

To this end, different approaches can be used, such as decision trees, Markov models and discrete event simulations ${ }^{12}$. Differences in effects between particle therapy and the conventional alternative treatment are expected, especially a gain in longterm effects. Therefore, in the case of particle therapy, cost-effectiveness and/or cost-utility analysis using a Markov model approach is recommended. Markov models are based on a series of 'states' that a patient can occupy at a given point in time.

Time elapses explicitly within a Markov model (Figure 1$)^{13}$, with the probability of patients occupying a given state assessed over series of discrete time periods, called cycles. The length of these cycles will depend on the nature of the disease and 
Table 1: Requirements needed for different types of economic evaluations.

\begin{tabular}{lllll}
\hline & \multicolumn{3}{ll}{ Are both costs (input) and effects (output) examined? } \\
\hline & & no & yes \\
Is there a comparison between & yes & Only effects & Only costs & \\
two alternatives? & Outcome & Cost & Cost-outcome description \\
& description & description & \\
& no & Efficacy or Effectiveness & Cost analysis & Full economic evaluation*
\end{tabular}

* Cost-benefit analysis, cost-utility analysis or cost-effectiveness analysis (source: Drummond et $\mathrm{al}^{15}$ )

Table 2: Types of economic evaluation.

\begin{tabular}{lll} 
Method & Costs & Effects \\
\hline Cost-minimization analysis (CMA) & Monetary units $(€)$ & No difference in effects \\
Cost-effectiveness analysis (CEA) & Monetary units $(€)$ & Natural units (life years gained, point blood pressure, etc) \\
Cost-utility analysis (CUA) & Monetary units $(€)$ & Utilities and Quality Adjusted Life Years (QALYs) \\
Cost-benefit analysis (CBA) & Monetary units $(€)$ & Monetary units ( $(€)$
\end{tabular}

(source: Drummond et $\mathrm{al}^{15}$ )

the interventions being evaluated, but could be a month or a year. Each state in the model has a cost associated with it and, for cost-utility analysis, a utility value. The time during which the average patient occupies the various states in the model will, when weighted by the relevant cost or utility, be used to calculate the expected costs and outcomes. The speed with which patients move between the states in the model is determined by a set of transition probabilities. More detailed information about Markov modeling can be found elsewhere ${ }^{12,14}$.

\section{RESULTS}

Out of 773 identified papers, only 13 papers were dealing with economic aspects of particle therapy ${ }^{15-26,27}$. An update of the review until June $1^{\text {st }} 2008$ yielded another 4 references ${ }^{28-31}$, resulting in a total of 17 references. Eight papers described aspects of the workload, staffing levels, setting up and operating costs of a proton therapy facility ${ }^{15,17-20,22,24,25}$. Five publications discussed several cost aspects regarding PT $15,21,23,30,31$. Under the auspices of ENLIGHT, one of these references ${ }^{23}$, presented the main methodological aspects of designing a model to assess treatment cost per protocol, whereas in another paper ${ }^{30}$ some results retrieved from this cost simulation 


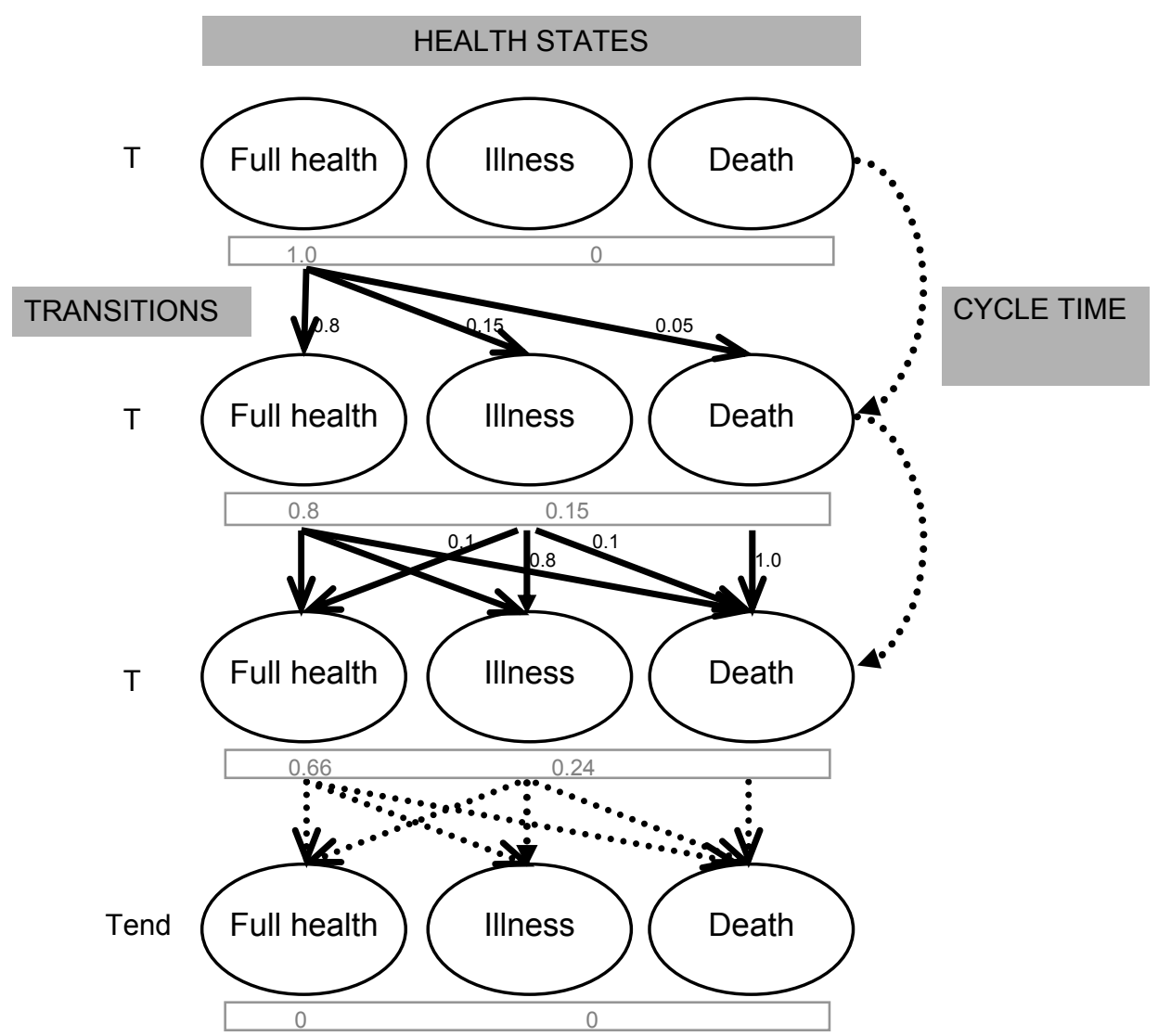

Figure 1 Bubble-diagram representation of a three-state Markov model. Patients are either in a state of full health, illness or death, represented by the ovals. Each row represents one cycle of a predefined time period. Transitions between the states are represented as arrows between the ovals, and transition probabilities appear beside the arrows on first appearance. The proportion of patients in each state at the beginning of the cycle is shown in the box below the ovals. When a lifetime time horizon is used, the Markov process ends when all patients are dead ${ }^{18}$.

tool are presented. Only five papers reported on "cost-effectiveness" ${ }^{16,26-29}$. In addition, we found 6 references reporting on current PT projects within Europe ${ }^{32-37}$. Below, a summary of the above literature on costs and the cost-effectiveness of PT is described.

\subsection{Costs of particle therapy}

Five papers reported on the costs of PT ${ }^{15,21,23,30,31}$. Contrary to general assumption, radiotherapy $(\mathrm{RT})$, a highly technological discipline requiring expensive equipment and skilled personnel, is not an expensive treatment modality, consuming only about $5 \%$ of the costs of the global oncology budget ${ }^{21}$. Rather, innovative irradiation tech- 
niques - being more time-consuming and requiring more expensive equipment and often also highly qualified personnel - will inevitably turn out to be more expensive. The cost of proton therapy, estimated on average at $€ 25,000$ (range: $11,000-35,000$ ) is approximately 2.4 times higher than sophisticated photon beam treatments (IMRT) 15. However, Lievens and van den Bogaert ${ }^{21}$ discussed the relative impact of the higher costs of proton treatment from a broad perspective and concluded that from a global healthcare standpoint, the cost of proton therapy is by no means outrageous and should be no justification for denying potentially curative and less toxic treatments to patients.

\subsubsection{Factors influencing the cost of particle therapy}

The total cost per treatment (or by fraction) is dependent on the construction costs (capital) and the operational costs (personnel, utility, and maintenance)[15]. The cost of particle therapy is likely to be heavily influenced by the treatment protocol used, as well as aspects of service delivery, e.g. number of fractions, number of beams per fraction, operating hours per day, number of treatment rooms, quality assurance modalities, technical equipment required, etc. ${ }^{30,31}$ These factors are likely to differ between countries and also between different facilities within one country ${ }^{38}$. A costsimulation performed by the ENLIGHT-ESTRO economic group, based on data from five European projects ${ }^{32-35,37}$, calculated a mean total cost for exclusive carbon ion radiotherapy (using horizontal beams) of $€ 19,008$ (SD $€ 1,921)^{30}$. For the specific treatment protocols the mean total costs appeared $€ 14,399, € 21,286$ and $€ 21,491$ for lung cancer, chordoma and prostate cancer respectively. If using a gantry, the mean total cost are $€ 25,034$ (SD $€ 2,378$ ) and may increase for a specific treatment protocol e.g. paranasal cancers to $€ 26,136$. Using the same (updated) cost simulation tool including data on recruitment from the French ETOILE project only, the total C-ion treatment cost per patient varied from $€ 12.000$ to 28.000 according to the protocol modalities for a given operating scenario and recruitment ${ }^{31}$. In their results it was shown that the mean cost per patient may be dramatically reduced when reducing the mean number of sessions per protocol and the mean duration of each session. Perrier et $\mathrm{al}^{30}$ found in the sensitivity analysis that a variation of one minute for irradiation would change the mean cost by $4 \%$ using horizontal beams and $3.2 \%$ if using a gantry. Reducing the number of fractions from twenty to five would reduce the mean total cost from $€ 19,008$ to $€ 12,373^{30}$. In addition, the requirement of high-tech equipment such as a carbon-ion gantry or an online PET-CT will influence the cost of this new treatment modality. Finally, it should be acknowledged that - besides the actual cost of the treatment - the reimbursement system, also variable per country, will influence price setting ${ }^{5,15,21,23}$. 


\subsubsection{Reimbursement}

Historically, in most Western countries radiotherapy financing systems were retrospective; that is, reimbursement was based on the actual services and treatments provided. Given that the delivery of more (complex) services then translates into more income for the provider, an incentive towards performing more - and more complex - services can be found. However, in this era of increasing governmental awareness about ever-increasing health care expenditure, a gradual shift has been observed (at least in Europe) towards the more frequent implementation of prospective reimbursement systems ${ }^{39,40}$.

So how are PT centers actually being reimbursed? In most cases, reimbursement fees per treatment are negotiated with governmental or other insurance companies on the basis of preliminary treatment cost calculations and motivated by the assumed improvements in outcome - better local control, fewer side effects - expected from PT treatment as compared to optimal conventional treatment. In some situations, the investment costs have largely been covered by, for example, the (local) government or university. Finally, allowance of special reimbursements on a case by case basis appears feasible $^{41,42}$.

\subsection{Cost-effectiveness of particle therapy: current literature}

Only five papers reporting on the "cost-effectiveness" of proton therapy have been published $^{16,26-29}$, which are tabulated in Table 3 . The cost-effectiveness of proton therapy as compared to conventional RT for skull base chordoma ${ }^{28}$, prostate cancer ${ }^{26,29}$, childhood medullo blastoma ${ }^{26,27}$ and left side breast cancer ${ }^{16,26}$ was investigated. Four studies used a Markov model ${ }^{16,26,27,29}$ for their analysis using a Monte Carlo cohort simulation, whereas one study used a retrospective analysis of 10 patients ${ }^{28}$. Only one study performed probabilistic analysis ${ }^{29}$. All papers but Konski et $\mathrm{al}^{29}$ reported the incremental cost and effects. None of the studies performed an 'expected value of perferct information' (EVPI) analysis. Out of five papers, three were authored by Lundkvist et al. ${ }^{16,26,27}$. This Swedish research group investigated an economic evaluation model of proton radiation therapy for childhood medulloblastoma ${ }^{27}$, a cost-effectiveness analysis model for breast cancer[16], and a cost-utility analysis based on a Markov model for breast cancer, prostate cancer, head and neck cancer, and medulloblastoma ${ }^{26}$. The results of this latter study indicated that the average cost per QALY gained for these four types of cancer was about $€ 10,130$, whereas this was $€ 66,608$, $€ 3,811$ and $€ 26,776$ for breast cancer, $H \& N$ medulloblastoma and prostate cancer respectively. Konski et $\mathrm{al}^{29}$ showed that, the incremental costeffectiveness ratio of proton therapy for adenocarcinoma (intermediate risk) of the prostate compared to IMRT photon treatment was $\$ 63,578 /$ QALY. In addition, they reported that proton beam therapy would be the prefered treatment if patients receiv- 
ing IMRT would have a utility less than 0.85 . The remaining article investigated the cost-effectiveness of proton treatment for skull base chordomas ${ }^{28}$, with the results showing that the cost per life year gained (LYG) was $€ 7,692$. If society was willing to pay $€ 50,000$ for a QALY or a LYG (thresholds vary significantly between countries: $€ 20,000-100,000)$, proton treatment would be deemed cost-effective.

\section{DISCUSSION}

The current literature on cost-effectiveness is scarce, non-comparable, and largely not performed according to the standard health technology assessment criteria as described in the methods section of this article. Furthermore, due to a high level of uncertainty given the lack of data and many assumptions in all of the five studies mentioned above, these results should be interpreted with caution.

This lack of current evidence on clinical efficacy and cost-effectiveness of particle therapy, and the ongoing discussion whether it is required to perfom RCT's or not, emphasizes the need to investigate the efficiency of particle therapy in an adequate manner with a quality methodology. One preeminent method for this purpose is a model-based economic evaluation using a Markov model.

Although Konski et $\mathrm{al}^{29}$ and Lundkvist et $\mathrm{al}^{16,26,27}$ already designed a Markov model for the cost-effectiveness of particle therapy, the following limitations were found. First, the identified health states for prostate cancer between the different models of Konski et $\mathrm{al}^{29}$ and Lundkvist et $\mathrm{al}^{26}$ are not similar. In this latter study, health states such as 'healthy', 'chronic adverse events', 'acute adverse events'and 'death' were included in the model. In contrast, Konski et $\mathrm{al}^{29}$ included health states like 'alive (with and without desease progresion)' and 'death' in their model. Konski et al ${ }^{29}$ didn't take toxicity into account and it was assumed that the utilities for IMRT and proton therapy were similar (0.9). It is questionable if this assumption is justified, since the late toxicity grade seems to be lower after proton therapy as compared to photon treatment ${ }^{3}$. Moreover, as written in Lundkvist et $\mathrm{al}^{26}$ a $7 \%$ reduction in health utility can be expected after the occurrence of an adverse event. 


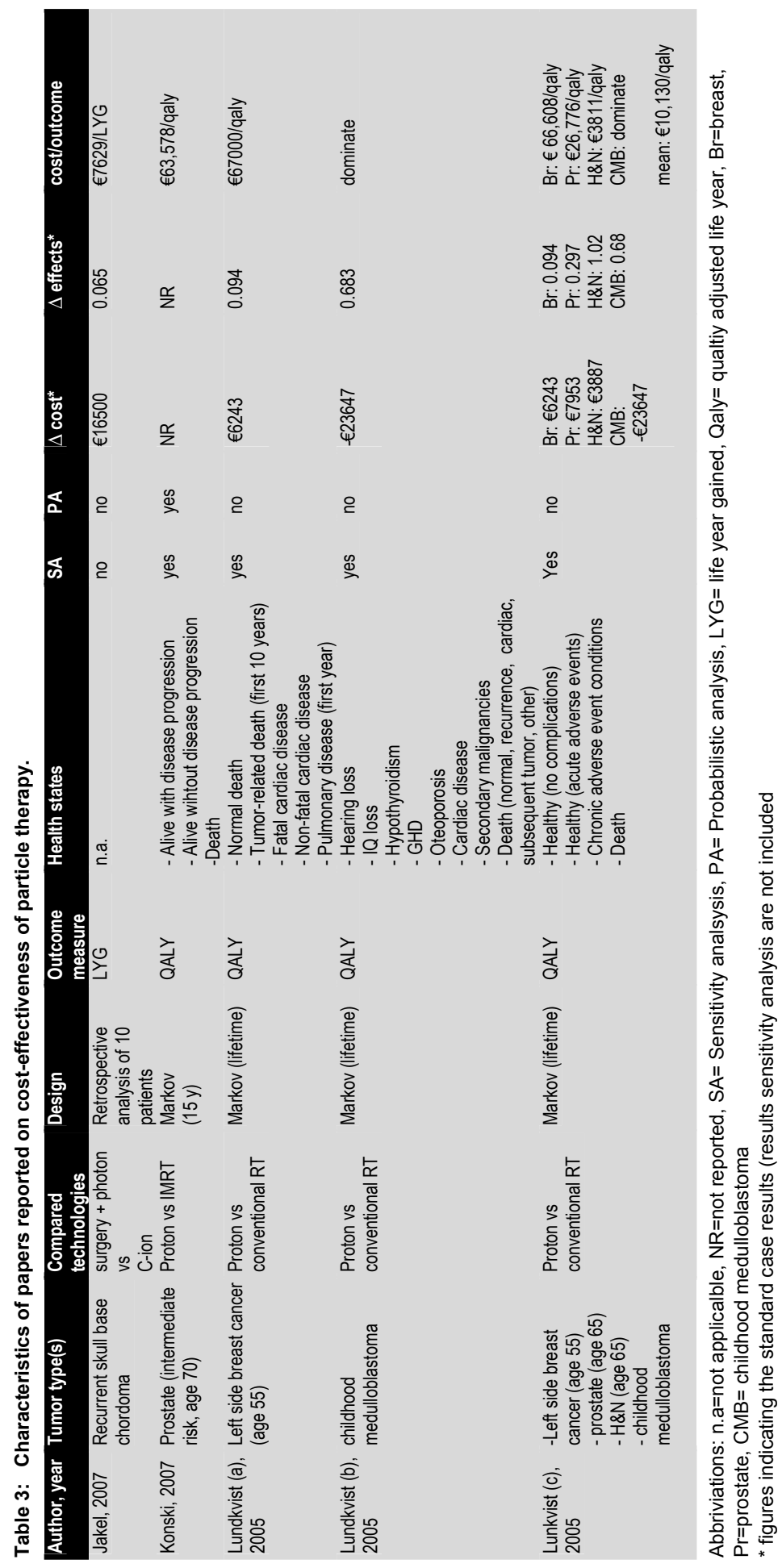


We assume that the different results retrieved from both Markov models on prostate cancer can mainly be ascribed to the fact that one model included toxicity while the other did not. With this comparison it will be obvious that it is of major importance to identify the most important health status for each group of patients.

Secondly, the ideal input parameters for a Markov model are pooled estimates from meta-analysis derived from phase III trials. Unfortunately, as described above, phase III trials in the field of particle therapy are very limited. However, one advantage of a model-based evaluation is that one can incorporate uncertainty into the model and then estimate, in light of this uncertainty, the probability that the new treatment will be cost-effective, e.g. such as only performed by Konski et $\mathrm{al}^{29}$. However, the authors of this latter publication failed to report the types of distributions and standard errors they have used for their probabilistic sensitivity analysis, and the cost and effect figures underlying the ICERS including confidence intervals, which makes it difficult to interpret the results. Thirdly, in addition, if a probabilistic analysis is performed, one can investigate whether it is worthwhile doing additional research in order to decrease uncertainty (according to the 'expected value of perfect information (EVPI) method) ${ }^{11}$. To our knowledge, such analysis has never been performed within the field of radiation oncology. With respect to critical assessment in health technology, results from an EVPI analysis show clearly for which parameters future research would be most valuable, e.g. whether it is worthwhile to investigate in clinical effectiveness or not. In such a modeling approach, the results from planning studies could be used as an input parameter as an alternative to data retrieved from phase III trials. One major advantage of using results from planning studies is that the focus can be on both tumor tissue and normal tissue. Based on the ALARA principle of positing radiation dose "as low as reasonably achievable", it can be convincingly shown (especially if endorsed by an international body) that the irradiation of normal tissue is lower with this new technology. This could be of major importance for the reimbursement of treatment costs by way of medical insurance. If this low dose can also be related to a lower risk of complications based on published validated dose response curves, then the argument would be made stronger yet. As in clinical studies, the best evidence will be reached if pooled estimates can be calculated from the results of single planning studies. In comparing these studies, however, the difficulty arises of reconciling the differences between radiotherapy departments of imaging systems and techniques, a result of the rapid evolution of new technologies in imaging as well as in target delineation (CT-PET, 4D, new algorithms, MRS, auto contouring, etc.). A viable option for improving comparisons of individual planning studies might be the development of a multicentric reference dataset of patients.

Determining long-term effects and costs is a substantial advantage in use of a Markov model. It is expected that in the long run, due to a decrease in the absorbed integral dose, fewer secondary cancers will be induced. Also, due to the expected decrease in side effects and increase in tumor control probability (TCP), it is ex- 
pected that long-term costs will decrease as a result of less hospitalization, less palliative care and improved quality of life. Therefore, it would be interesting to determine the probability of local tumor recurrence, treatment related morbidity at different toxicity levels and the time of appearance of the morbidity and the frequency of $G$ II progressing to G III after 5 or 10 years and then to G IV at 10-30 years. To our knowledge, none of the published studies on cost-effectiveness mentioned in this paper gives insight in the above.

Besides finding means for accurately calculating the cost-effectiveness of particle therapy, we might also consider the costs of particle therapy from a broader perspective, e.g. the health care perspective, taking the total treatment cost of a specific disease into account (incl. chemotherapy, hospitalization, palliative care, etc.) or the societal perspective, in which all costs will be considered, including productivity loss (cost from the time off from work) either by the patient of by family member(s). The latter perspective is especially relevant if it concerns younger patients (age<65). Let us, for example from the health care perspective, suppose that the total treatment cost of a patient with lung cancer (diagnosis, chemotherapy, biological therapy, surgery, conventional radiation) amounts to approximately $€ 100,000$, and that of this, $€ 10,000$ is consumed by conventional high-quality photon radiation. Assuming that the cost of proton radiation would be $€ 20,000$, implementing proton irradiation in the total treatment of a lung cancer patient would thus amount to an extra $€ 10,000$. This represents an increase of $10 \%$, rather than an increase of $100 \%$, meaning that the total cost of RT would remain relatively low.

Reimbursement plays an important role regarding the price-setting of PT. A gradual shift has been observed (at least in Europe) towards the more frequent implementation of prospective reimbursement systems ${ }^{39,40}$.

This means providers' revenues are determined before the costs are actually incurred. As a consequence, financial responsibility shifts more towards the providers, and a tendency to deliver fewer - or less complex - treatments is often found in prospective reimbursement systems. Besides, it has to be mentioned that there might be a risk of reduced effectiveness of treatment, i.e. in case of pressure in reducing the number of treatment sessions to increase the "profit margin". Because of the different stimuli towards practice found in retrospective and prospective financing systems, it has been suggested that a combination of both strategies within the one system might be the best way to promote the cost-effective delivery of high-quality treatment $^{5}$. This certainly also holds true for PT treatments, but how to optimally do so is an issue that remains unsolved.

Following the assignement of special reimbursements on a case by case basis, another approach,- which is more appealing from a conceptual point of view- would be to cover the investment costs and perhaps even the early running costs with a grant, as long as the treatment is considered investigational. The obtaining of such a grant could then be made conditional on performing the clinical research needed to gather 
objective effectiveness information. In a later stage, if PT treatment emerges as the superior treatment within well-defined clinical situations, cost-effectiveness analyses could then be performed in order to guide decisions about adequate reimbursement levels and for implementation into daily practice.

Regrettably, in many countries governmental research money is not easily available, which can make securing important investments such as those needed for installing a new PT facility seem impossible out of reach. Furthermore, waiting for effectiveness data to mature will take longer still. Thus, we should consider other approaches which have, in their early stages, provided some insight into the potential costeffectiveness of PT therapy.

In conclusion, there is little evidence yet on the cost-effectiveness of particle therapy. Given the implementation of particle treatments in an increasing number of centers, this evidence may be forthcoming, but it will take many years for firm results to emerge.

For now, adequate reimbursement is necessary to support such innovative yet costly treatments, both in the phase of clinical research as well as once introduced into daily practice. However, some questions remain: How to define and to obtain this reimbursement? How to proceed while awaiting evidence? How can the RT community prepare and cooperate for the forthcoming influx of this evidence?

We recommend these items be brought to the forefront of discussions in international RT working groups, in particular with professional associations like ASTRO and ESTRO. Meanwhile, model-based economic evaluations, with synthesized data, including probabilistic and EVPI analysis, may help us to transparantly report uncertainty and to gain evidence-based insight into potential solutions. To determine the impact of this new modality treatment on the global oncology budget, these evaluations must be performed from a broad perspective, at least from a health care point of view rather than a narrow perspective such as a hospital point of view.

Further research is needed, however, not only into the effectiveness of particle treatments but also their costs and the optimal means of defining reimbursement levels. It is imperative that research be streamlined in such a way that data on all these questions will become available in the coming years. To this end, international cooperation (and guidelines) is of the utmost importance. 


\section{8 | Part 3: New technologies- Chapter 8}

\section{REFERENCES}

1. Glimelius, B, Ask, A, Bjelkengren, G, et al. Number of patients potentially eligible for proton therapy. Acta Oncol 2005;44:836-849.

2. Brada, M, Pijls-Johannesma, M, De Ruysscher, D. Proton therapy in clinical practice: current clinical evidence. Journal of clinical oncology 2007;25:965-970.

3. Lodge, M, Pijls-Johannesma, M, Stirk, L, Munro, AJ, De Ruysscher, D, Jefferson, T. A systematic literature review of the clinical and cost-effectiveness of hadron therapy in cancer>. Radiother Oncol 2007;83:110-122.

4. Olsen, DR, Bruland, OS, Frykholm, G, Norderhaug, IN. Proton therapy - A systematic review of clinical effectiveness. Radiother Oncol 2007;83:123-132.

5. Lievens, Y. [Valorisation of high technology radiotherapy in Europe]. Cancer radiotherapie 2006;10:361-369.

6. Glimelius, B, Montelius, A. Proton beam therapy - Do we need the randomised trials and can we do them?>. Radiother Oncol 2007;83:105-109.

7. Lawrence, T, Petrelli, N, Li, B, Galvin, J. Think Globally, Act Locally. Journal of Clinical Oncology 2007;25:921-923.

8. Bentzen, SM. Randomized controlled trials in health technology assessment: overkill or overdue? Radiother Oncol 2008;86:142-147.

9. Goitein, M, Cox, JD. Should randomized clinical trials be required for proton radiotherapy? J Clin Oncol 2008;26:175-176.

10. Suit, H, Kooy, H, Trofimov, A, et al. Should positive phase III clinical trial data be required before proton beam therapy is more widely adopted? No. Radiother Oncol 2008;86:148-153.

11. Claxton, K, Sculpher, M, Drummond, M. A rational framework for decision making by the National Institute For Clinical Excellence (NICE). Lancet 2002;360:711-715.

12. Briggs, A, Sculpher, M, Claxton, K. Decision modelling for health economic evaluation. Oxford: Oxford University Press. 2006.

13. Naimark, D, Krahn, MD, Naglie, G, Redelmeier, DA, Detsky, AS. Primer on medical decision analysis: Part 5--Working with Markov processes. Med Decis Making 1997;17:152-159.

14. Drummond, M, McGuuire, A. Economic Evaluation in Health Care: merging theory with practice. Oxford: Oxford University Press. 2006.

15. Drummond M, Sculpher M, Torrance G, et al: Methods for the Economic Evaluation of Health Care Programmes (ed third). Oxford, Oxford medical publications, 2005

16. Goitein, M, Jermann, M. The relative costs of proton and X-ray radiation therapy. Clinical oncology (Royal College of Radiologists (Great Britain)) 2003;15:S37-50.

17. Lundkvist, J, Ekman, M, Ericsson, SR, Isacsson, U, Jonsson, B, Glimelius, B. Economic evaluation of proton radiation therapy in the treatment of breast cancer. Radiother Oncol 2005;75:179-185.

18. Cohilis, P, Jongen, Y. Some factors influencing the cost of a hospital based proton therapy centre. Strahlenther Onkol 1999;175:102-104.

19. Fleurette, F, Charvet Protat, S. [Proton and neutron radiation in cancer treatment: clinical and economic outcomes]. Bull-Cancer-Radiother. 1996;83:223s-227s.

20. Khoroshkov, VS, Onosovsky, KK, Klenov, GI, Zink, S. Moscow hospital-based proton therapy facility design. Am J Clin Oncol 1994;17:109-114.

21. Knapp, FF, Jr., Mirzadeh, S. The continuing important role of radionuclide generator systems for nuclear medicine. Eur-J-Nucl-Med 1994;21:1151-1165.

22. Lievens, Y, Van den Bogaert, W. Proton beam therapy: too expensive to become true? Radiother Oncol 2005;75:131-133.

23. Maughan, RL, Brambs, B, Porter, AT, Forman, JD. The cost-effectiveness of mixed beam neutronphoton radiation therapy in the treatment of adenocarcinoma of the prostate. Strahlenther Onkol 1999;175:104-107. 
24. Pommier, P, Zucca, L, Naslund, I, et al. Light ion facility projects in Europe: methodological aspects for the calculation of the treatment cost per protocol. Radiother Oncol 2004;73:S183-185.

25. Battaglia, G, B, E, B, M. Financial analysis of the activities of the hadron therapy centre. Part VI - costs and time schedule. The Tera Project and the Center for Oncology Hadrotherapy, INFN-LNF-Divisione Ricerca. 1994.

26. Orecchia, R, Zurlo, A, Loasses, A, et al. Particle beam therapy (hadrontherapy): basis for interest and clinical experience. Eur J Cancer 1998;34:459-468.

27. Lundkvist, J, Ekman, M, Ericsson, SR, Jonsson, B, Glimelius, B. Proton therapy of cancer: potential clinical advantages and cost-effectiveness. Acta Oncol 2005;44:850-861.

28. Lundkvist, J, Ekman, M, Ericsson, SR, Jonsson, B, Glimelius, B. Cost-effectiveness of proton radiation in the treatment of childhood medulloblastoma. Cancer 2005;103:793-801.

29. Jakel, O, Land, B, Combs, SE, Schulz-Ertner, D, Debus, J. On the cost-effectiveness of Carbon ion radiation therapy for skull base chordoma. Radiother Oncol 2007;83:133-138.

30. Konski, A, Speier, W, Hanlon, A, Beck, JR, Pollack, A. Is proton beam therapy cost effective in the treatment of adenocarcinoma of the prostate? J Clin Oncol 2007;25:3603-3608.

31. Perrier, L, Combs, S, Auberger, T, et al. A Decision-Making Tool for a Costly Innovative Technology: the case of Carbon lon radiotherapy. Journal d"Economie Médicale 2007;25:367-380.

32. Pommier, P, Gueye, N, Buron, C, et al. Medico-economic and Spatial Modelization for decision making in innovative therapies: application to light ions therapy projects in Europe. Radiotherapy and Oncology 2006;81:S411.

33. Amaldi, U. CNAO--The Italian Centre for Light-Ion Therapy. Radiother Oncol 2004;73 Suppl 2:S191201.

34. Bajard, M, De Conto, JM, Remillieux, J. Status of the "ETOILE" project for a French hadrontherapy centre. Radiother Oncol 2004;73 Suppl 2:S211-215.

35. Griesmayer, E, Auberger, T. The status of MedAustron. Radiother Oncol 2004;73 Suppl 2:S202-205.

36. Haberer, T, Debus, J, Eickhoff, H, Jakel, O, Schulz-Ertner, D, Weber, U. The Heidelberg lon Therapy Center. Radiother Oncol 2004;73 Suppl 2:S186-190.

37. Huybrechts, M, Obyn, C, Gailly, J, Mambourg, F, Vinck, I, Ramaekers, D. Hadrontherapy. HealthTechnology Assessment (HTA), Brussels: Federaal Keniscentrum voor de Gezondheidszorg (KCE ). KCE reports 67A. 2007.

38. Lambin, P, Fekkers, H, Daniels, E, et al. Euregional Ion Therapy Institute. Maastricht: Business plan. 2005.

39. Ploquin, NP, Dunscombe, PB. The cost of radiation therapy. Radiother Oncol 2008;86:217-223.

40. Lievens, Y, Van den Bogaert, W, Rijnders, A, Kutcher, G, Kesteloot, K. Palliative radiotherapy practice within Western European countries: impact of the radiotherapy financing system? Radiother Oncol 2000;56:289-295.

41. Slotman, BJ, Cottier, B, Bentzen, SM, Heeren, G, Lievens, Y, van den Bogaert, W. Overview of national guidelines for infrastructure and staffing of radiotherapy. ESTRO-QUARTS: work package 1. Radiother Oncol 2005;75:349-354.

42. Tsujii, H, Mizoe, J, Kamada, T, et al. Clinical Results of Carbon Ion Radiotherapy at NIRS. J Radiat Res (Tokyo) 2007;48 Suppl A:A1-A13.

43. Tsujii, H, Mizoe, JE, Kamada, T, et al. Overview of clinical experiences on carbon ion radiotherapy at NIRS. Radiother Oncol 2004;73 Suppl 2:S41-49. 



\section{CHAPTER}

General Discussion and

Future Perspectives 



\section{GENERAL DISCUSSION AND FUTURE PERSPECTIVES}

The aim of the work described in this thesis was to get insight in the balance between intensive radiotherapy (RT) and Quality of Life (QoL) to enable adequate decision making in lung cancer patients. For this latter, the role of new technologies in lung cancer and their cost-effectiveness was investigated as well.

Since survival after curative treatment with RT is the most frequently used study endpoint, we included only patients treated with curative intent. In the last decade several studies demonstrated that the combination of chemotherapy (CT) and RT provided better median and long term survival than RT alone, especially if delivered concurrently. However the influence of time factors in this combined modality treatment, such as the timing of RT and the overall treatment time, remained unclear. Accelerated proliferation of tumor clonogens is a well-recognized cause of failure after radiotherapy and chemotherapy in several malignancies ${ }^{1-7}$. The first part of this thesis therefore focused at analyzing these time factors. We chose to investigate LDSCLC, because this is a rapidly growing cancer where both chemotherapy and chest radiotherapy play a role ${ }^{8-13}$. We found that early delivering of Chest RT as compared to late Chest RT delivery in the combined modality treatment with CT leads to higher survival rates and that significantly higher 5-year survival (>20\%) can be achieved when the start of any treatment until the end of radiotherapy (SER) is less than 30 days (Fig. 1).

Apart from optimizing time factors, the total radiation dose to the tumor could contribute to better survival rates in lung cancer. However, increasing the radiation dose will inevitably lead to a higher incidence of side-effects, which may consequently lead to a decrease of QoL. The same holds true for combined concurrent modality treatment: it increases long-term survival, but at the expense of a higher incidence of severe esophagitis ${ }^{14-17}$. In order to allow proper balancing between expected benefits and drawbacks of aggressive therapy, we examined the effects of a given treatment on the quality of life (QoL), as described in part 2 of this thesis. We found that intensive high-dose radiotherapy or concurrent chemo-radiation in the treatment of lung cancer seems to be a well tolerated treatment option with preservation of QoL (Fig.1).

New radiotherapy treatment modalities such as stereotactic body RT (SBRT) and irradiation with charged particles, seem to be promising, since these techniques allow better sparing of normal tissues. Until now, mainly inoperable stage I NSCLC patients have been treated with these new technologies. For this reason, we studied for this part of our work the results of NSCLC stage I patients in order to determine the effectiveness of particle therapy as compared to SBRT and conventional 3 dimensional conformal RT (3DRT). 


\section{4 | Chapter 9}

Since particle therapy is a relatively expensive treatment modality and the high costs can be considered as the main stagnation factor in the start-up phase of new particle centers, we also examined the current evidence of cost-effectiveness of particle therapy as described in part 3 of this thesis. We found that there is little or no evidence yet on the cost-effectiveness of particle therapy and that model-based economic evaluations performed at least from a health care perspective may help us to gain evidence-based insight into the efficiency of particle therapy (Fig.1). 


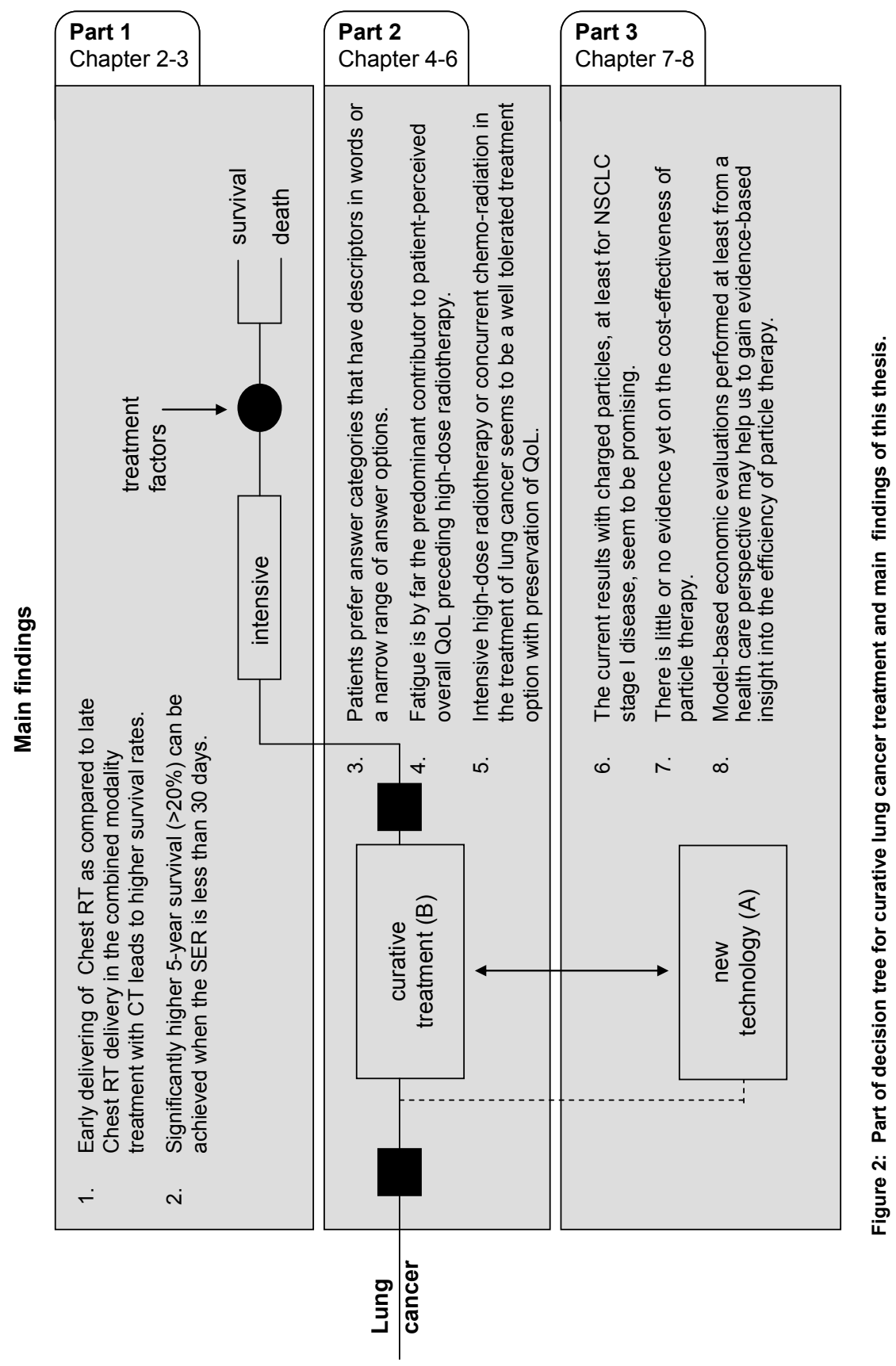




\section{PART 1}

\section{Determination of radiation treatment factors that improve survival}

In the first part of this thesis we found that the timing of chest RT in the combined modality treatment with concurrent platinum based chemotherapy (CT) influences the survival rate in favor of early deliverance of chest RT ( $<30$ days after start CT). However, this result should be interpreted with caution since this result is based on a comparison with only one trial that did not use platinum-based CT. Besides, the studies with the highest reported dose intensity of chemotherapy seem to have the highest survival rates and show the largest differences between early and late chest radiotherapy ${ }^{18-21}$. The same studies also report the highest compliance rates. It seems that in order to have a benefit of early chest radiotherapy, an effective systemic treatment as well as a two-year survival of at least $30 \%$ should be achieved. This may be explained by the fact that the beneficial effect of early chest radiation can only be observed in those patients in a good general condition, thoroughly staged, and thus well compliant for an intensive, but effective chemotherapy regimen combined with chest radiotherapy. However, this hypothesis cannot be proven by the available data. In the studies with an overall chest radiation treatment time of fewer than 30 days ${ }^{18-22}$, the five-year survival rates significantly indicated the benefits of early chest radiotherapy, whereas at the two-year rates no effect was seen of early chest radiotherapy.

The finding that the relation between the timing of chest radiation and the start of any treatment until the end of radiotherapy (SER) and the 5-year survival was highly significant, supports the occurrence of accelerated proliferation of tumor clonogens during concurrent chemotherapy and radiotherapy. As a consequence, the best survival of patients with fast proliferating tumors may be achieved when 2 or 3 full-dose cycles of chemotherapy and chest radiotherapy are delivered before accelerated tumor cell proliferation starts (approximately 30 days in case of LD-SCLC), whether triggered by chemotherapy or by chest radiation.

Keeping all caveats for comparing local toxicity between studies in mind, severe pneumonitis and severe esophagitis were not significantly different between early and late chest radiotherapy, whether non-platinum chemotherapy was administered during chest radiotherapy or not ${ }^{18-23}$, but a trend for a higher incidence of pneumonitis and severe esophagitis was observed when delivering chest radiotherapy early or with a short SER. This is in line with previous findings, indicating that intensifying chemotherapy and radiotherapy regimens increases the cytotoxic effects on rapidly proliferating cells, including the mucosa ${ }^{1,3}$. It also may be that larger radiation fields were used when irradiation was carried out during the first or the beginning of the second chemotherapy cycles, at a time when the primary tumor and the lymph node 
metastases were still larger than after later chemotherapy cycles. In addition, when the overall treatment time of chest radiation was less than 30 days ${ }^{18,19,21,22}$, severe local toxicity was not significantly different between early and late chest radiotherapy or between a short or a long SER.

Compliance rates of the studies with concurrent chemo-radiotherapy, except for the early arm of the studies of Skarlos et al and Spiro et al, were high (87\% to $100 \%)^{18-}$ ${ }^{20}$, which implies that these schedules are well tolerated.

When comparing the results between trials, which are from a methodological point of view not highly valid due to a large heterogeneity, we found that a 5-year survival of more than $20 \%$ might be achieved when the SER was less than 30 days, with an upper bound of the $95 \% \mathrm{Cl}$ of 90 days. In the absence of for that purpose designed randomized trials or meta-analysis based on individual patient data, the exact value of the optimal SER thus remains uncertain.

\section{PART 2}

\section{QoL of lung cancer patients before, during and after intensive radiotherapy}

Using the disease-specific quality of life measure EORTC-QLQ-C30, our results indicated that, of all QoL domains/subscales, fatigue was by far the predominant contributor to patient-perceived overall QoL in lung patients preceding high-dose radiotherapy. In fact, fatigue alone explained a greater proportion of variance in overall QoL than the combined functional and symptoms subscales of all other domains (somatic, psychological, social). This finding suggests that, in our study population, the impact of fatigue on overall QoL largely overruled the impact of any other functional and symptom subscales.

In a subsequent study the evolution of QoL over time during and after high-dose intensive RT was investigated. QoL decreased shortly after RT but increased again within 3 months after the end of RT to the pre-treatment level. This short decrease of global quality of life could be explained by the side effects of RT. In addition, the majority of the symptoms and functioning scores did not change much over time, and if so, it was reversible, and occurred only shortly after finishing radiotherapy. However, in some symptoms and functioning scores, a persistent worsening was seen: Physical state, accomplishing daily tasks (role) and cognitive functioning, dyspnea, dysphagia, and hoarseness, detoriated after RT, and were significantly worse at the end of the 18 months follow-up period. Adjusted for potential confounders, esophagitis appeared to be a strong predictor for QoL. However, the number of patients who suffer from severe esophagitis appeared to be very low (12\%). More apparent predictors for QoL found in this study appeared to be gender, tumor stage, and fatigue. This 
latter finding is not surprising, as fatigue is identified as the most predominant factor that influences overall QoL ${ }^{24-26}$. In addition, the more persistent worsening of some symptoms and functioning scores may be caused by the occurrence of relapse. However, since we did not take this into account in our analysis we are not able to draw any conclusion on this.

Our results showed that performance status (PS) did not significantly contribute to the overall QoL. Earlier publications that reported on the correlation between PS and QoL are ambiguous ${ }^{24,27,28}$. It could be that the PS pales into insignificance, due to the fact that we adjusted for fatigue, a variable which is identified as a strong potential confounder.

In summary, our results demonstrated that high-dose RT or concurrent chemoradiation for the treatment of lung cancer only had a temporary and small influence on QoL. After fatigue, acute esophagitis had the largest impact on QoL. The reduction of this side effect, e.g. by implementing innovations in radiotherapy of lung cancer such as intensity-modulated RT, tomotherapy, stereotactic body RT (SBRT) or particle therapy ${ }^{29,30}$, could further improve the $\mathrm{QoL}$ of lung cancer patients.

\section{PART 3}

\section{Decision making on new innovative technologies: effectiveness, QoL and costs}

While new health technologies are ultimately aimed at improving treatment outcome, the ultimate goal is to improve efficiency as compared to standard care ${ }^{31}$. Typically, the extra cost of a new intervention (compared to the standard treatment) must be balanced with its additional gain in clinical effect (i.e., better local control and/or survival, decreased morbidity/better QoL).

Future radiotherapy treatment modalities like particle therapy seem promising regarding both improvement of survival and quality of life. Available data demonstrate that particle therapy in general and specifically proton therapy is a safe and feasible treatment modality, thus having successfully passed the phase I stage of an experimental therapy.

Because of its theoretical advantages, protons and light ions show great promise for they may enable lowering the dose to the normal tissues, allow radiation dose escalation and hence yield increased tumor control probabilities ${ }^{32}$. On top of that, light ions, having a higher biological effectiveness than protons or photons, may further increase the effectiveness of charged particles beyond that of protons ${ }^{33}$.

We showed however, that for most indications, no firm conclusions could be drawn from the published literature about the clinical efficacy of this treatment modality ${ }^{34-36}$. 
For lung cancer it was reported that proton therapy leads to equivalent outcome as modern photon therapy ${ }^{34}$ in selected patients. Nevertheless, recently presented results at an international conference on particle therapy are promising. NSCLC patients stage III treated with chemotherapy and concurrent proton beam therapy had decreased toxicity when compared with $\mathrm{IMRT}^{37}$. There was significantly less fatigue in patients treated with protons when all grades of fatigue were compared $(p=0.04)$ as well as when the analysis was limited to grade 2 or greater fatigue $(p<0.001)$. The authors suggest that the greater conformality with proton therapy compared with IMRT may decrease myelotoxicity. Distant disease is a major problem in advanced lung cancers and by limiting the myelosuppression associated with radiation it may be possible to decrease the number of patients who require a break from chemotherapy or a dose reduction. It may be possible to escalate the concurrent dose of chemotherapy or allow other agents (such as biologicals) to be added to the chemoradiation, which could potentially improve outcomes. Pneumonitis is another important toxicity that could potentially be decreased with the use of protons. From the results of a subsequent study presented at the same conference, it appears that protons do reduce the irradiated volume of normal lung and have the potential to decrease the rate of radiation pneumonitis compared with $\mathrm{IMRT}^{38}$. These results suggest that protons could reduce the risk of radiation pneumonitis and may again allow further dose escalation, thereby improving local control.

Because of above promising recent results as well as the overwhelming theoretical data on the beneficial properties of protons and light ions ${ }^{39-48}$, further investment in the infrastructure needed to perform large trials in patients with different lung cancer stages, is warranted. Also, studies should investigate the possible role of $\mathrm{C}$-ion therapy in SCLC, as also in this histology, local tumor failure occurs in at least $30 \%$ of patients ${ }^{49}$. Nakano and collegues ${ }^{50}$ showed in their study on bulky cervical cancer (stage IIIb and IVa) that the intratumoral $\mathrm{pO}_{2}$ status during $\mathrm{C}$-ion therapy had no influence on either disease-free survival or local control probability. Hence, it would be worthwhile to investigate in clinical studies with hypoxic tumors like SCLC or advanced NSCLC.

In the meantime, the complexity introduced by tumor motion must be recognized ${ }^{51-54}$. However, as usually using a scattered beam, the particle beam treats the whole target volume, the chance of missing the target will reduce as compared with IMRT were only a portion of the target is treated per beam. Given that the cost of particle therapy is considerably higher than that of conventional radiotherapy with photons, it is necessary to establish whether these higher costs are worthwhile in light of the expected advantages. Since decisions need to be made on whether or not to invest in particle centers, evidence is needed on whether, and for which indications, particle therapy is a cost-effective treatment modality. This puts the introduction of new (expensive) irradiation treatments such as particle therapy in a difficult situation: firm data on effectiveness can only be obtained after the implementation and clinical 
evaluation of the treatment, which in the case of radiotherapy often implies that important capital investments must be made. Yet data on effectiveness will only become available years after the necessary investment in equipment, buildings and highly qualified personnel. As mentioned above, effectiveness data on PT treatments are lacking; regardless, sufficient financing is necessary to perform clinical research and ensure the eventual dissemination of evidence-based treatments into clinical practice.

This lack of current evidence on clinical efficacy and cost-effectiveness of particle therapy emphasizes the need to investigate the efficiency of particle therapy in an adequate manner with a quality methodology. One preeminent method for this purpose in the short term is a model-based economic evaluation using a Markov model.

\section{Final discussion and conclusion}

We found in this thesis that time-factors play an important role in the treatment of lung cancer. QoL appeared to be an important clinical outcome, valuable for the evaluation of treatments. High-dose radiotherapy seems well tolerated in the treatment of lung cancer with preservation of QoL. As described in chapter 1, the assessment of QoL can be done with generic or disease-specific self-reporting questionnaires. In this thesis, we have chosen for disease-specific questionnaires due to the high sensitivity to clinical relevant outcomes. However, in economic evaluations as suggested above, these measures have major limitations. The main disadvantage, from the economist's viewpoint, is that they do not give comprehensive measures of QoL and therefore cannot be used to compare the cost-effectiveness of programs in different disease areas ${ }^{55}$. So, regarding this statement, could we conclude that we made the wrong choice in selecting a disease-specific measurement tool to assess QoL? According to Dowie ${ }^{56}$, all choices can be justified as long as a decisional perspective, rather than a knowledge perspective, is taken on QoL when we are concerned with health care decision making. Once knowing the available options, it can be determined what is needed to know about QoL in order to establish the best option. Three levels of decision making can be distinguished, (1) national or regional level (macro level), (2) healthcare facility level (meso level) and (3) healthcare provider level (micro level). Regarding the impact of economic evaluations on decision making, van Velden et al $^{57}$ concluded that the most pronounced influence of economic evaluations on health care decision making was found at the meso and macro level. As the physicians responsibility is providing optimal patient care, the influence of economic arguments at the micro level decision making appeared to be minor ${ }^{57}$. This indicates a different perspective of interest between the present work described in this thesis (micro level) and the recommendations that were made for future research, that is economic evaluations for particle therapy (macro level). The findings presented in this thesis were derived from a micro level perspective, where medical 
decision making from individual health care professionals aimed to improve a specific treatment for a specific patient. On the other hand, decision making at the macro level concerns decision makers with a national or more regional healthcare perspective who coordinate activities outside healthcare organizations. They are responsible for the availability of effective and affordable healthcare programs for the whole populations ${ }^{57}$. In case of particle therapy, these people have to decide whether particle therapy should be reimbursed or not. For this, economic evaluations could be support this medical decision making. Nevertheless, for decisions on micro level, these economic evaluations are of minor importance.

\section{FUTURE PERSPECTIVES}

Nowadays several treatment options with various different radiation dose schedules delivered single or in a combined treatment modality, are available for the treatment of lung cancer. However, the exact influence of many factors, like the most adequate timing, the start of any treatment until the end of radiotherapy (SER), total irradiation dose, dose intensity of chemotherapy and the most adequate combinations of chemo regimens remains unclear, although most of these factors are highly correlated with survival outcome. Currently, a meta-analysis on individual patient data is under way to explore all these factors and to test earlier findings.

As many treatment possibilities are available and under development, determining the cost-effectiveness or cost-utility of those treatments has to be investigated.

Healthcare advances such as new drugs, devices, or screening and diagnostic tests must demonstrate safety and efficacy to be approved for clinical use. However, because of rising healthcare costs and limited budgets, questions may remain about their value. Cost-effectiveness analysis is one approach to determining value and refers to a method for assessing the costs and health benefits of an intervention. Assuming that health budgets cannot meet all of the possible demands, costeffectiveness evaluation can assist decision-makers in allocating resources to maximize the net public health benefit from choosing among options in the care of patients.

In this thesis, the QoL was determined using a disease-specific QoL questionnaire, the EORTC QLQ-C30. For decision making on the micro level, this appears the most optimal QoL assessment tool, since we were interested in determining also small changes in clinical (relevant) outcome. A generic questionnaire is less sensitive and could fail to measure disease specific disabilities. To be able to adeqately perform a cost-utility analysis, we measured generic preference-based QoL (utilities), the EuroQoL $5 D^{75}$. Preliminary results are described below. 
It is recommended to investigate in the effectiveness of new advanced radiotherapy technologies, like stereotactic body RT (SBRT) and particle therapy (PT). The results of SBRT for stage I NSCLC seem promising since recently reported local control rates are in excess of $85-95 \%{ }^{58-65}$. The technique of SBRT is characterized by the use of accurate repositioning during treatment simulation and delivery and ablative doses that are typically delivered in three to five fractions. In our review ${ }^{30}$ we found similar local control rates with $\mathrm{C}$-ions, although the latter are probably accompanied by less side effects. In this era of evidence-based medicine, although this current results of PT are promising, more evidence is required before PT can become internationally the standard treatment for (subsets of) lung cancer patients. It would be worthwhile to know which subgroups of patients would profit most from particle therapy, specifically from C-ions and to obviously explore clinical evidence of the benefits of PT compared to SBRT and conventional treatment with 3DRT and surgery.

In a prelimininary analysis in MAASTRO, we examined the cost-effectiveness of proton therapy and carbon-ion therapy as opposed to 3DRT and SBRT in the treatment of inoperable stage I NSCLC patients. A threshold of $€ 80,000 / Q A L Y$ was applied, according to the guideline of the Council for Public Health and Health Care advised the Ministry of Health, Welfare and Sport ${ }^{66}$.

Transition probabilities were derived for each cycle from published studies that reported sufficient data for SBRT ${ }^{58-65}$, proton therapy ${ }^{67-69}$ and carbon-ions ${ }^{70-73}$. For 3DRT transition probabilities were derived from one recent publication ${ }^{74}$. Utility scores were collected in a cross-sectional survey, using the EuroQoL $5 D^{75}$. Uncertainty was handled probabilistically, which means that distributions were assigned to the parameters to reflect the uncertainty in the estimation of that parameter. Preliminary results showed that the mean expected total health care costs per patient over a 5 year period were $€ 23,121$ for CRT, for SBRT $€ 13,650$, for protons $€ 22,184$ and for carbon-ions $€ 20,222$. The mean expected quality adjusted life-years (QALYs) were $1.80,2.47,2.39$ and 2.53 respectively (Figure 2 ).

SBRT was found to be less expensive and more effective than CRT and proton therapy. Carbon-ions were both more costly and more effective than SBRT. In this circumstance an incremental cost-effectiveness ratio (ICER) needs to be calculated to examine whether the extra costs are worth the extra effects. This resulted in an ICER of $€ 107,639$ per QALY for carbon-ions as opposed to SBRT. However, as can be seen in Figure 2, these estimates are surrounded by considerable uncertainty. 


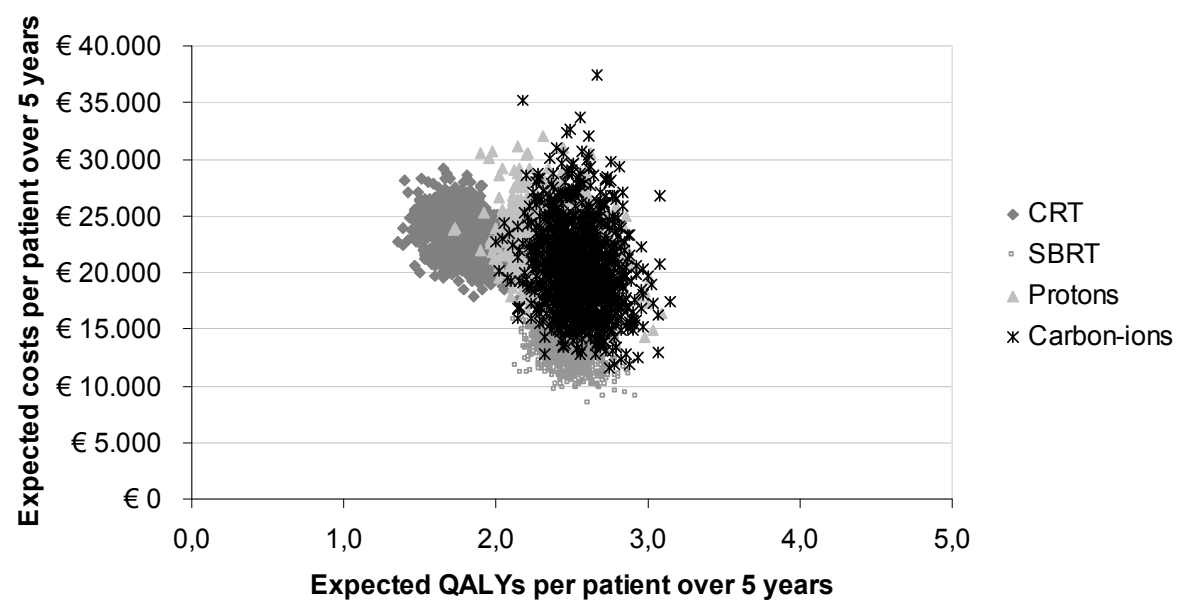

Figure 2. Preliminary results of probabilistic senstivity analysis.

The cost-effectiveness acceptability curves (CEAC) presented in Figure 3, illustrate this uncertainty surrounding the estimate of cost- effectiveness. A CEAC shows the probability that an intervention is cost-effective compared with the alternative, given the observed data, for a range of maximum monetary values that a decision-maker might be willing to pay for a particular unit change in outcome.

The probabilistic sensitivity analysis showed that for a ceiling ratio $€ 80,000$, carbonions had the highest probability of being cost-effective $(43 \%)$, followed by SBRT (42\%) and protons (15\%) (Figure 3). The result of CRT was $0 \%$ and therefore not visible in Figure 3.

Our preliminary results indicate that particle therapy is a potentially cost-effective treatment modality in lung cancer. Taking again the directions of the Dutch Council for Public Health and Health Care into account, the preliminary results of the above described cost-effectiveness study are above the threshold of $€ 80,000 / Q A L Y$. However, the differences are small and surrounded by considerable uncertainty. 


\section{$154 \mid$ Chapter 9}

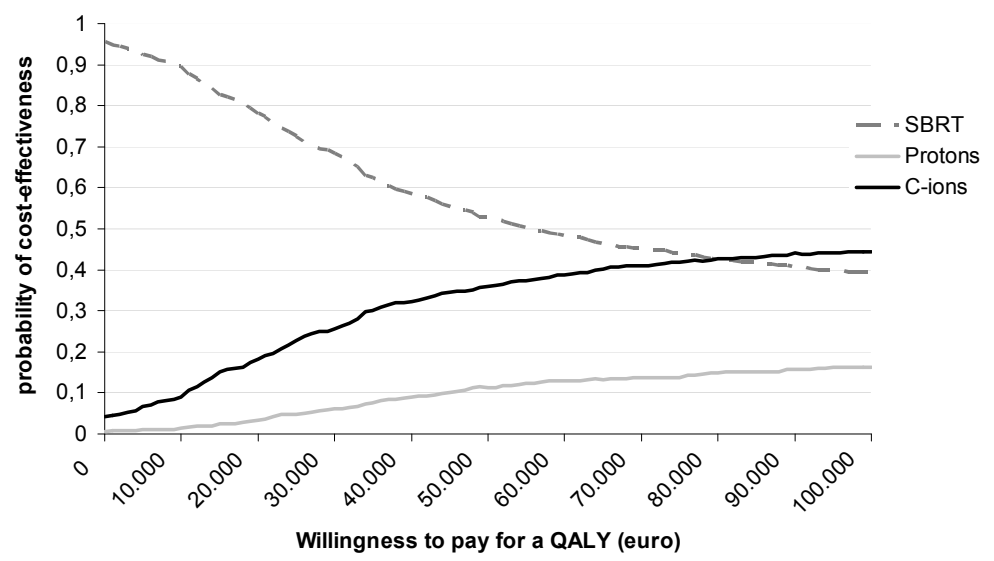

Figure 3. Cost-effectiveness acceptability curves for the three treatment modalities.

More analyses will be performed in the project. First, more advanced statistical techniques will be applied to synthesize the available evidence. Second, other comparators will be examined (IMRT and protons for prostate, surgery for operable NSCLC patients). Third, the cost-effectiveness of particle therapy will be assessed for other disease stages such as stage III NSCLC. Finally, expected value of perfect information analyses will be performed to support decisions on whether it is worthwhile to perform additional reseach and for which parameters more evidence is most valuable. 


\section{REFERENCES}

1. Bentzen SM: Repopulation in radiation oncology: perspectives of clinical research. Int $\mathrm{J}$ Radiat Biol 79:581-5, 2003

2. Bentzen SM, Thames HD: Clinical evidence for tumor clonogen regeneration: interpretations of the data. Radiother Oncol 22:161-6, 1991

3. Bernier J, Bentzen SM: Altered fractionation and combined radio-chemotherapy approaches: pioneering new opportunities in head and neck oncology. Eur J Cancer 39:560-71, 2003

4. Davis AJ, Tannock IF: Tumor physiology and resistance to chemotherapy: repopulation and drug penetration. Cancer Treat Res 112:1-26, 2002

5. Davis AJ, Tannock JF: Repopulation of tumour cells between cycles of chemotherapy: a neglected factor. Lancet Oncol 1:86-93, 2000

6. Withers HR, Taylor JM, Maciejewski B: The hazard of accelerated tumor clonogen repopulation during radiotherapy. Acta Oncol 27:131-46, 1988

7. Wu L, Tannock IF: Repopulation in murine breast tumors during and after sequential treatments with cyclophosphamide and 5-fluorouracil. Cancer Res 63:2134-8, 2003

8. Stupp R, Monnerat C, Turrisi AT, 3rd, et al: Small cell lung cancer: state of the art and future perspectives. Lung Cancer 45:105-17, 2004

9. Warde P, Payne D: Does thoracic irradiation improve survival and local control in limited-stage smallcell carcinoma of the lung? A meta-analysis. J Clin Oncol 10:890-5, 1992

10. Bunn PA, Jr., Carney DN: Overview of chemotherapy for small cell lung cancer. Semin Oncol 24:S769-S7-74, 1997

11. De Ruysscher D, Vansteenkiste J: Chest radiotherapy in limited-stage small cell lung cancer: facts, questions, prospects. Radiother Oncol 55:1-9, 2000

12. Kelly K: New chemotherapy agents for small cell lung cancer. Chest 117:156S-162S, 2000

13. Pignon JP, Arriagada R, Ihde DC, et al: A meta-analysis of thoracic radiotherapy for small-cell lung cancer. N Engl J Med 327:1618-24, 1992

14. Zatloukal P, Petruzelka L, Zemanova M, et al: Concurrent versus sequential chemoradiotherapy with cisplatin and vinorelbine in locally advanced non-small cell lung cancer: a randomized study. Lung Cancer 46:87-98, 2004

15. Fournel P, Robinet G, Thomas $P$, et al: Randomized phase III trial of sequential chemoradiotherapy compared with concurrent chemoradiotherapy in locally advanced non-small-cell lung cancer: Groupe Lyon-Saint-Etienne d'Oncologie Thoracique-Groupe Francais de Pneumo-Cancerologie NPC 95-01 Study. J Clin Oncol 23:5910-7, 2005

16. Furuse K, Fukuoka M, Kawahara M, et al: Phase III study of concurrent versus sequential thoracic radiotherapy in combination with mitomycin, vindesine, and cisplatin in unresectable stage III nonsmall-cell lung cancer. J Clin Oncol 17:2692-9, 1999

17. Vokes EE, Crawford J, Bogart J, et al: Concurrent chemoradiotherapy for unresectable stage III nonsmall cell lung cancer. Clin Cancer Res 11:5045s-5050s, 2005

18. Jeremic B, Shibamoto $\mathrm{Y}$, Acimovic $\mathrm{L}$, et al: Initial versus delayed accelerated hyperfractionated radiation therapy and concurrent chemotherapy in limited small-cell lung cancer: a randomized study. J Clin Oncol 15:893-900, 1997

19. Murray N, Coy P, Pater JL, et al: Importance of timing for thoracic irradiation in the combined modality treatment of limited-stage small-cell lung cancer. The National Cancer Institute of Canada Clinical Trials Group. J Clin Oncol 11:336-44, 1993

20. Takada M, Fukuoka M, Kawahara M, et al: Phase III study of concurrent versus sequential thoracic radiotherapy in combination with cisplatin and etoposide for limited-stage small-cell lung cancer: results of the Japan Clinical Oncology Group Study 9104. J Clin Oncol 20:3054-60, 2002 


\section{6 | Chapter 9}

21. Skarlos DV, Samantas E, Briassoulis E, et al: Randomized comparison of early versus late hyperfractionated thoracic irradiation concurrently with chemotherapy in limited disease small-cell lung cancer: a randomized phase II study of the Hellenic Cooperative Oncology Group (HeCOG). Ann Oncol 12:12318, 2001

22. Spiro SG, James LE, Rudd RM, et al: Early compared with late radiotherapy in combined modality treatment for limited disease small-cell lung cancer: a London Lung Cancer Group multicenter randomized clinical trial and meta-analysis. J Clin Oncol 24:3823-30, 2006

23. Perry MC, Herndon JE, 3rd, Eaton WL, et al: Thoracic radiation therapy added to chemotherapy for small-cell lung cancer: an update of Cancer and Leukemia Group B Study 8083. J Clin Oncol 16:24667, 1998

24. Dagnelie PC, Pijls-Johannesma MC, Lambin P, et al: Impact of fatigue on overall quality of life in lung and breast cancer patients selected for high-dose radiotherapy. Ann Oncol 18:940-4, 2007

25. Langendijk JA, Aaronson NK, de Jong JM, et al: Prospective study on quality of life before and after radical radiotherapy in non-small-cell lung cancer. J Clin Oncol 19:2123-33, 2001

26. Langendijk JA, Aaronson NK, ten Velde GP, et al: Pretreatment quality of life of inoperable non-small cell lung cancer patients referred for primary radiotherapy. Acta Oncol 39:949-58, 2000

27. Siddiqui F, Kohl R, Swann S, et al: Gender differences in pretreatment quality of life in a prospective lung cancer trial. J Support Oncol 6:33-9, 2008

28. Jacot W, Colinet B, Bertrand D, et al: Quality of life and comorbidity score as prognostic determinants in non-small-cell lung cancer patients. Ann Oncol, 2008

29. Schild SE, Bogart JA: Innovations in the radiotherapy of non-small cell lung cancer. J Thorac Oncol 1:85-92, 2006

30. Pijls-Johannesma M, Grutters JP, Lambin P, et al: Particle therapy in lung cancer: Where do we stand? Cancer Treat Rev 34:259-67, 2008

31. Bentzen SM: Randomized controlled trials in health technology assessment: overkill or overdue? Radiother Oncol 86:142-7, 2008

32. Chang JY, Liu HH, Komaki R: Intensity modulated radiation therapy and proton radiotherapy for nonsmall cell lung cancer. Curr Oncol Rep 7:255-9, 2005

33. Orecchia R, Zurlo A, Loasses A, et al: Particle beam therapy (hadrontherapy): basis for interest and clinical experience. Eur J Cancer 34:459-68, 1998

34. Brada M, Pijls-Johannesma M, De Ruysscher D: Proton therapy in clinical practice: current clinical evidence. Journal of clinical oncology 25:965-70, 2007

35. Lodge M, Pijls-Johannesma M, Stirk L, et al: A systematic literature review of the clinical and costeffectiveness of hadron therapy in cancer>. Radiother Oncol 83:110-22, 2007

36. Olsen DR, Bruland OS, Frykholm G, et al: Proton therapy - A systematic review of clinical effectiveness. Radiother Oncol 83:123-32, 2007

37. Komaki R, Sejpal S, Wei X, et al: Reduction of bone marrow suppression for patients with stage III NSCLC treated by proton and chemotherapy compared with IMRT and chemotherapy, PTCOG 47. Jacksonville, Florida, 2008, pp 14 (O10)

38. Chang J, xx, xx: Does Proton Beam Radiotherapy (PBT) Reduce Treatment Related Pneumonitis (TRP) Compared to Intensity Modulated Radiation Therapy (IMRT) in Patients with Locally Advanced Non-Small Cell Lung Cancer (NSCLC) Treated with Concurrent Chemotherapy?, PTCOG 47. Jacsonville, Florida, 2008, pp xx (Oxx)

39. Krengli M, Hug EB, Adams JA, et al: Proton radiation therapy for retinoblastoma: comparison of various intraocular tumor locations and beam arrangements. Int J Radiat Oncol Biol Phys 61:583-93, 2005

40. St Clair WH, Adams JA, Bues M, et al: Advantage of protons compared to conventional X-ray or IMRT in the treatment of a pediatric patient with medulloblastoma. Int J Radiat Oncol Biol Phys 58:727-34, 2004

41. Weber DC, Trofimov AV, Delaney TF, et al: A treatment planning comparison of intensity modulated photon and proton therapy for paraspinal sarcomas. Int J Radiat Oncol Biol Phys 58:1596-606, 2004 
42. Baumert BG, Lomax AJ, Miltchev $\mathrm{V}$, et al: A comparison of dose distributions of proton and photon beams in stereotactic conformal radiotherapy of brain lesions. Int J Radiat Oncol Biol Phys 49:1439-49, 2001

43. Zurlo A, Lomax A, Hoess A, et al: The role of proton therapy in the treatment of large irradiation volumes: a comparative planning study of pancreatic and biliary tumors. Int $\mathrm{J}$ Radiat Oncol Biol Phys 48:277-88, 2000

44. Miralbell R, Crowell C, Suit HD: Potential improvement of three dimension treatment planning and proton therapy in the outcome of maxillary sinus cancer. Int J Radiat Oncol Biol Phys 22:305-10, 1992

45. Cozzi L, Fogliata A, Lomax A, et al: A treatment planning comparison of 3D conformal therapy, intensity modulated photon therapy and proton therapy for treatment of advanced head and neck tumours. Radiother Oncol 61:287-97, 2001

46. Lee $\mathrm{CH}$, Tait $\mathrm{D}$, Nahum AE, et al: Comparison of proton therapy and conformal X-ray therapy in nonsmall cell lung cancer (NSCLC). Br J Radiol 72:1078-84, 1999

47. Fogliata A, Bolsi A, Cozzi L: Critical appraisal of treatment techniques based on conventional photon beams, intensity modulated photon beams and proton beams for therapy of intact breast. Radiother Oncol 62:137-45, 2002

48. Isacsson $\mathrm{U}$, Montelius $\mathrm{A}$, Jung $\mathrm{B}$, et al: Comparative treatment planning between proton and $\mathrm{X}$-ray therapy in locally advanced rectal cancer. Radiother Oncol 41:263-72, 1996

49. Turrisi AT, 3rd, Kim K, Blum R, et al: Twice-daily compared with once-daily thoracic radiotherapy in limited small-cell lung cancer treated concurrently with cisplatin and etoposide. N Engl J Med 340:26571, 1999

50. Nakano T, Suzuki Y, Ohno T, et al: Carbon beam therapy overcomes the radiation resistance of uterine cervical cancer originating from hypoxia. Clin Cancer Res 12:2185-90, 2006

51. Bush DA, Slater JD, Garberoglio C, et al: A technique of partial breast irradiation utilizing proton beam radiotherapy: comparison with conformal x-ray therapy. Cancer J 13:114-8, 2007

52. Engelsman M, Rietzel E, Kooy HM: Four-dimensional proton treatment planning for lung tumors. Int $\mathrm{J}$ Radiat Oncol Biol Phys 64:1589-95, 2006

53. Kang $\mathrm{Y}$, Zhang $\mathrm{X}$, Chang JY, et al: 4D Proton treatment planning strategy for mobile lung tumors. Int $\mathrm{J}$ Radiat Oncol Biol Phys 67:906-14, 2007

54. Mori S, Endo M, Komatsu S, et al: Four-dimensional measurement of lung tumor displacement using 256-multi-slice CT-scanner. Lung Cancer 56:59-67, 2007

55. Drummond M, Sculpher M, Torrance G, et al: Methods for the Economic Evaluation of Health Care Programmes (ed third). Oxford, Oxford medical publications, 2005

56. Dowie J: Decision validity should determine whether a generic or condition-specific HRQOL measure is used in health care decisions. Health Econ 11:1-8, 2002

57. van Velden ME, Severens JL, Novak A: Economic evaluations of healthcare programmes and decision making: the influence of economic evaluations on different healthcare decision-making levels. Pharmacoeconomics 23:1075-82, 2005

58. Lagerwaard FJ, Haasbeek CJ, Smit EF, et al: Outcomes of risk-adapted fractionated stereotactic radiotherapy for stage I non-small-cell lung cancer. Int J Radiat Oncol Biol Phys 70:685-92, 2008

59. Nyman J, Johansson KA, Hulten U: Stereotactic hypofractionated radiotherapy for stage I non-small cell lung cancer--mature results for medically inoperable patients. Lung Cancer 51:97-103, 2006

60. Onishi H, Shirato H, Nagata $\mathrm{Y}$, et al: Hypofractionated stereotactic radiotherapy (HypoFXSRT) for stage I non-small cell lung cancer: updated results of 257 patients in a Japanese multi-institutional study. J Thorac Oncol 2:S94-100, 2007

61. Uematsu M, Shioda A, Suda A, et al: Computed tomography-guided frameless stereotactic radiotherapy for stage I non-small cell lung cancer: a 5-year experience. Int J Radiat Oncol Biol Phys 51:666-70, 2001

62. Koto M, Miyamoto T, Yamamoto N, et al: Local control and recurrence of stage I non-small cell lung cancer after carbon ion radiotherapy. Radiother Oncol 71:147-56, 2004 


\section{Chapter 9}

63. Zimmermann FB, Geinitz H, Schill S, et al: Stereotactic hypofractionated radiotherapy in stage I (T1-2 NO MO) non-small-cell lung cancer (NSCLC). Acta Oncol 45:796-801, 2006

64. Xia T, Li H, Sun Q, et al: Promising clinical outcome of stereotactic body radiation therapy for patients with inoperable Stage I/II non-small-cell lung cancer. Int J Radiat Oncol Biol Phys 66:117-25, 2006

65. Hoyer M, Roed H, Traberg Hansen A, et al: Phase II study on stereotactic body radiotherapy of colorectal metastases. Acta Oncol 45:823-30, 2006

66. RvZ-rapport: Zinnige en duurzame zorg: advies uitgebracht door de Raad voor de Volksgezondheid en Zorg aan de minister van Volksgezondheid, Welzijn en Sport. Zoetermeer, 2006

67. Bush DA, Slater JD, Shin BB, et al: Hypofractionated proton beam radiotherapy for stage I lung cancer. Chest 126:1198-203, 2004

68. Hata M, Tokuuye K, Kagei K, et al: Hypofractionated high-dose proton beam therapy for stage I nonsmall-cell lung cancer: preliminary results of a phase I/Il clinical study. Int J Radiat Oncol Biol Phys 68:786-93, 2007

69. Nihei K, Ogino T, Ishikura S, et al: High-dose proton beam therapy for Stage I non-small-cell lung cancer. Int J Radiat Oncol Biol Phys 65:107-11, 2006

70. MacManus R, Hicks R, Matthews J, et al: ? Proc Am Soc Clin Oncol Abstract, 2002

71. Miyamoto $\mathrm{T}$, Baba $\mathrm{M}$, Sugane $\mathrm{T}$, et al: Carbon ion radiotherapy for stage I non-small cell lung cancer using a regimen of four fractions during 1 week. J Thorac Oncol 2:916-26, 2007

72. Miyamoto T, Baba M, Yamamoto N, et al: Curative treatment of Stage I non-small-cell lung cancer with carbon ion beams using a hypofractionated regimen. Int J Radiat Oncol Biol Phys 67:750-8, 2007

73. Miyamoto $\mathrm{T}$, Yamamoto $\mathrm{N}$, Nishimura $\mathrm{H}$, et al: Carbon ion radiotherapy for stage I non-small cell lung cancer. Radiother Oncol 66:127-40, 2003

74. Lagerwaard FJ, Senan S, van Meerbeeck JP, et al: Has 3-D conformal radiotherapy (3D CRT) improved the local tumour control for stage I non-small cell lung cancer? Radiother Oncol 63:151-7, 2002

75. EuroQol-Group: EuroQol--a new facility for the measurement of health-related quality of life. Health Policy 16:199-208, 1990 
SUMMARY 
Radiotherapy (RT) is one of the most important curative and palliative treatment modalities for both non-small cell lung cancer (NSCLC) and small-cell lung cancer (SCLC). RT has made significant progress in the past years, due to more advanced technologies, better staging, insights in radiation (molecular) biology and combined modality approaches. It has consistently been shown that increased local tumor control by concurrent chemo-radiation (CTR) is translated in increased survival. Moreover, increasing the radiation dose leads to an increased local tumor control. Radiation doses of $60 \mathrm{~Gy}$ in 30 fractions of $2 \mathrm{~Gy}$ are effective in controlling post-operative microscopic disease, more than $100 \mathrm{~Gy}$ might be necessary to sterilize the tumor as a whole. Theoretically, a dose of $>84 \mathrm{~Gy}$ is necessary to obtain a chance for a definite local control in small tumors of $50 \%$. However, delivery of high-dose RT requires restrictions of the total tumor dose and tumor volume, since higher doses to the normal tissue will increase the risk of complications of the surrounding normal tissues. This will thus lead to more treatment related side effects which are expected to result in a reduction of a patients' Quality of Life (QoL). Since overall survival is improving, QoL is increasingly being recognized as a very important endpoint to evaluate the treatment effect. Worldwide, many research groups are investigating RT or CTR for lung cancer, with the aim to improve survival with preservation of a patients' QoL. The aim of this thesis is to examine curative radiation treatment for lung cancer in order to get insight in the balance between the beneficial and adverse effects of intensive radiotherapy both with respect to survival and QoL. The ultimate goal is to enable adequate decision making. For this latter, the role of new technologies in lung cancer is also investigated (Chapter1).

\section{Part 1}

The first chapters are dedicated to treatment factors that may have influence on the long-term survival. In Chapter $\mathbf{2}$ we investigated the most effective way of combining chest radiotherapy with chemotherapy for patients with Limited Disease (LD) -SCLC in order to improve long-term survival. We found that the timing of chest RT in the combined modality treatment with concurrent platinum based chemotherapy (CT) influences the survival rate in favor of early deliverance of chest RT ( $<30$ days after start CT). Besides, the studies with the highest reported dose intensity of chemotherapy seem to have the highest survival rates and show the largest differences between early and late chest radiotherapy. The same studies also report the highest compliance rates. It seems that in order to have a benefit of early chest radiotherapy, an effective systemic treatment as well as a two-year survival of at least $30 \%$ should be achieved. In Chapter $\mathbf{3}$ we analyzed the time factors for combined chemotherapy and radiotherapy in more detail, in order to obtain long-term survival of patients with LD-SCLC. We found that the relation between the timing of chest radiation and the start of any treatment until the end of radiotherapy (SER) and the 5-year survival was highly significant. This supports the occurrence of accelerated proliferation of tumor 
clonogens during concurrent chemotherapy and radiotherapy. As a consequence, the best survival of patients with fast proliferating tumors may be achieved when 2 or 3 full-dose cycles of chemotherapy and chest radiotherapy are delivered before accelerated tumor cell proliferation starts (approximately 30 days in case of LD-SCLC), whether triggered by chemotherapy or by chest radiation. Keeping all caveats for comparing local toxicity between studies in mind, severe pneumonitis and severe esophagitis were not significantly different between early and late chest radiotherapy, whether non-platinum chemotherapy was administered during chest radiotherapy or not, but a trend for a higher incidence of pneumonitis and severe esophagitis was observed when delivering chest radiotherapy early or with a short SER. In general, compliance rates of the studies with concurrent chemo-radiotherapy were high $(87 \%$ to $100 \%$ ), which implies that these schedules are well tolerated.

\section{Part 2}

In Part 2 of this thesis several QoL issues are discussed regarding lung cancer patients treated with radiotherapy. The aim of the study described in Chapter $\mathbf{4}$ was to determine the preferences for and acceptability of existing Quality of Life (QoL) questionnaires among cancer patients. Based on criteria like the percentage of missing values, items indicated by patients as being difficult and the preferences of patients for answer options, generally used questionnaires were examined. We found that patients prefer answer categories that have descriptors in words or a narrow range of answer options (preference for a 4-point scale rather than a 10-point scale). In Chapter 5 we evaluated the relative impact of different QoL domains/subscales on overall QoL in lung cancer patients preceding radiotherapy. Fatigue appeared by far to be the predominant contributor to patient-perceived overall QoL in lung patients preceding high-dose radiotherapy. In fact, fatigue alone explained a greater proportion of variance in overall QoL than the combined functional and symptoms subscales of all other domains (somatic, psychological, and social). This finding suggests that, in our study population, the impact of fatigue on overall QoL largely overruled the impact of any other functional and symptom subscales. Next to it we also analyzed the evolution of QoL in patients with lung cancer selected for curative radiotherapy or chemoradiation which we described in Chapter 6. Our results demonstrated that high-dose RT or concurrent chemo-radiation for the treatment of lung cancer only had a temporary and small influence on QoL. After fatigue, acute esophagitis had the largest impact on QoL.

\section{Part 3}

The final two chapters are dedicated to future possibilities of new radiotherapy technologies and the impact on lung cancer treatment regarding survival and QoL. Since from a theoretical point of view, charged particles should lead to superior results 
compared to photons, in Chapter 7 we searched for clinical evidence that protons or $\mathrm{C}$-ions are really beneficial to patients with lung cancer. We showed however, that for most indications, no firm conclusions could be drawn from the published literature about the clinical efficacy of this treatment modality. For lung cancer it was reported that proton therapy leads to equivalent outcome as modern photon therapy in selected patients. Because of above promising recent results as well as the overwhelming theoretical data on the beneficial properties of protons and light ions, further investment in the infrastructure needed to perform large trials in patients with different lung cancer stages, is warranted. Finally, Chapter $\mathbf{8}$ focuses on different costing issues related to particle therapy in order to detect future needs and to recommend a strategy that allows for the evidence-based determination of the cost-effectiveness of particle therapy as compared to conventional radiotherapy. Due to the present lack of current evidence on clinical efficacy and cost-effectiveness of particle therapy, we recommend performing model-based economic evaluations using a Markov model to investigate the efficiency of particle therapy in an adequate manner with a quality methodology.

Finally, areas for future research are discussed in Chapter 9. One of these areas includes a meta-analysis on individual patient data that is currently under way to explore the influence of many factors on survival, like the most adequate timing, the start of any treatment until the end of radiotherapy (SER), total irradiation dose, dose intensity of chemotherapy and the most adequate combinations of chemo regimens. Also, as many treatment possibilities are available and under development, determining the cost-effectiveness or cost-utility of those treatments has to be investigated. More specific, we recommend investigating in the effectiveness of new advanced radiotherapy technologies, like stereotactic body RT (SBRT) and particle therapy (PT). 
\begin{tabular}{l|l} 
& $\mathbf{6 3}$
\end{tabular}

\section{SAMENVATTING}


Radiotherapie (RT) speelt een belangrijke rol in de behandeling van zowel nietkleincellige (NSCLC) als kleincellige (SCLC) longkanker. De afgelopen jaren is op het gebied van RT een aanzienlijke vooruitgang geboekt, mede door de ontwikkeling van geavanceerde technologieën, betere tumor stadiëring, meer inzicht in (moleculaire) radiobiologie en in de combinatie van verschillende behandelingen. Zo is bijvoorbeeld duidelijk aangetoond dat een gecombineerde behandeling van chemotherapie en radiotherapie resulteert in een toename van de lokale tumor controle met als gevolg een betere overleving. De bestralingsdosis van $60 \mathrm{~Gy}$ in 30 fracties vanaf $2 \mathrm{~Gy}$ blijkt effectief voor de ziektecontrole van postoperatieve microscopische ziekteuitbreiding, maar er lijkt toch een dosis van meer dan 100 Gy noodzakelijk te zijn om de tumor in zijn geheel te kunnen vernietigen. Theoretisch is een dosis van >84 Gy noodzakelijk voor $50 \%$ kans op een definitieve locale controle bij kleine tumoren. Echter, het toedienen van hoge dosis RT wordt beperkt door een aantal beperkingen met betrekking tot de totale tumordosis en het tumorvolume, omdat een hogere bestraalde dosis op het gezonde weefsel het complicatierisico van het gezonde weefsel doet toenemen. Daardoor kunnen dan meer behandelingsgerelateerde neveneffecten optreden, die naar verwachting de kwaliteit van leven $(\mathrm{KvL})$ van de patiënt nadelig beïnvloeden. Als er vooruitgang wordt geboekt in de overleving, is het belangrijk om KvL te evalueren als gevolg van de behandeling.

Dit proefschrift beoogt meer inzicht te krijgen in de curatieve behandeling van longkanker met RT waarbij de positieve en negatieve effecten van intensieve RT worden onderzocht met betrekking tot zowel overleving als KvL. Het uiteindelijke doel is om met hulp van de bevindingen in dit proefschrift op een adequatere wijze medische beslissingen te kunnen nemen. Daarom zal ook de rol van nieuwe technologieën in longkanker worden meegenomen (Hoofdstuk 1).

\section{Deel 1}

De eerste hoofdstukken beschrijven de behandelingsfactoren die van invloed kunnen zijn op de lange termijn overleving. In Hoofdstuk 2 wordt gekeken naar wat de meest effectieve manier is, m.b.t. verbetering van lange termijn overleving, om thoracale radiotherapie (TRT) te combineren met chemotherapie (CT) bij patiënten met kleincellige longkanker met een beperkte ziekteuitbreiding (LD-SCLC). We vonden dat vroegtijdige ( $<30$ dagen na start van CT) TRT in de gecombineerde behandeling met platinum-based CT de overleving verbeterde. Daarnaast bleek dat de studies met de hoogst gerapporteerde dosisintensiteit van de CT de hoogste overlevingspercentages hadden en tevens het grootste verschil in overlevingspercentage tussen vroege en late toediening van RT. In dezelfde studies werden bovendien de hoogste percentages therapietrouw gerapporteerd. Uit deze resultaten zou opgemaakt kunnen worden dat om de voordelen van vroege toediening van RT tot uiting te zien komen, men afhankelijk is van een effectieve systemische behandeling, waarbij de pa- 
tiënten in een relatief goede conditie moeten verkeren ( 2 jaar overleving van tenminste $30 \%$ ). In Hoofdstuk 3 worden vervolgens de tijdsfactoren voor een gecombineerde behandeling met CT en BRT meer gedetailleerd geanalyseerd. We vonden een aanzienlijke significante relatie tussen het tijstip van de start van een behandeling tot aan het einde van de RT (SER) en de 5 -jaar overleving. Dit resultaat ondersteunt de hypothese dat er een versnelde celdeling optreedt na de start van cytotoxische therapie. Hiermee rekening houdend zou de beste overleving van patiënten met snel groeiende tumoren worden bereikt indien 2 of 3 cycli CT en TRT worden toegediend voor de start van deze versnelde celdeling (ongeveer 30 dagen in het geval van LD-SCLC). De lokale toxiciteit tussen groepen die behandeld werden met een korte of lange SER bleek niet significant te verschillen, er was echter een trend zichtbaar dat meer bestralingsgeïnduceerde longontsteking en slokdarmontsteking werd gezien in de groep met een korte SER. Over het algemeen blijken de percentages therapietrouw hoog te zijn in de studies waarbij CT en TRT gelijktijdig wordt toegediend ( $87 \%-100 \%)$, wat suggereert dat de behandeling goed tolereerbaar is.

\section{Deel 2}

In deel 2 van dit proefschrift worden verschillende aspecten van kwaliteit van leven $(\mathrm{KvL})$ bij longkankerpatiënten die radiotherapie ondergaan besproken. Het doel van de studie beschreven in Hoofdstuk 4 was om de voorkeur en de aanvaardbaarheid van bestaande KvL vragenlijsten te bepalen bij kankerpatiënten. Gebaseerd op voorafgestelde criteria zoals het percentage niet ingevulde items, het aantal door de patient moeilijk bevonden vragen en de voorkeur van de patiënt voor bepaalde categorieën, hebben we de meest gangbare KvL vragenlijsten voorgelegd aan een groep patiënten met longkanker. $\mathrm{Na}$ analyse bleek dat patiënten de voorkeur gaven aan voorgeschreven antwoordcategorieën met een zo kort mogelijke antwoord-range. Op basis van deze resultaten verwachtten we dat indien in toekomstig onderzoek naar $\mathrm{KvL}$ rekening wordt gehouden met deze voorkeur van de patiënt, de vragenlijsten beter zullen worden ingevuld en de analyses beter kunnen worden uitgevoerd. In Hoofdstuk 5 hebben we vervolgens de relatieve impact van verschillende KvL domeinen/subschalen geëvalueerd met betrekking tot de globale KvL bij longkankerpatiënten voor aanvang van radiotherapie. Het bleek dat binnen deze patiëntengroep de mate van vermoeidheid de meest overheersende rol speelt in de door de patiënt waargenomen globale KvL. Feitelijk bleek vermoeidheid afzonderlijk een groter deel van de globale $\mathrm{KvL}$ te voorspellen dan de totale combinatie van functionele en symptoomschalen van andere $\mathrm{KvL}$ domeinen (somatisch, psychologisch, sociaal). Deze bevinding suggereert dat binnen onze studiepopulatie, de impact van vermoeidheid op de globale $\mathrm{KvL}$ grotendeels de impact van iedere andere functionele en symptoom subschaal overheerst. Daarnaast hebben we ook nog gekeken naar het verloop van de KvL bij patiënten die curatief behandeld worden met radiotherapie of chemo- 
radiotherapie, zoals beschreven in Hoofdstuk 6. De resultaten laten zien dat de invloed van hoge dosis radiotherapie of gelijktijdige chemoradiotherapie in de behandeling van longkanker op de $\mathrm{KvL}$ beperkt is en slechts van tijdelijke aard. Ook hier kwam vermoeidheid als sterkste voorspeller van de algemene KvL naar voren, gevolgd door acute slokdarmontsteking.

\section{Deel 3}

De laatste 2 hoofdstukken richten zich op nieuwe technologieën binnen de radiotherapie in de behandeling van longkanker, waarbij vooral wordt gekeken naar het verschil in effecten ten opzichte van de huidige radiotherapiebehandeling in termen van overleving en de kwaliteit van leven. Omdat vanuit een theoretisch oogpunt, deeltjestherapie (protonen en koolstofionen) superieure resultaten verwacht worden in vergelijking met de huidige bestraling met fotonen, hebben we in Hoofdstuk 7 onderzocht of er wetenschappelijk bewijsmateriaal is, dat de klinische behandeling van longkanker met protonen en koolstofionen effectiever is dan een behandeling met fotonen. Het bleek echter, dat voor de meeste indicaties, geen duidelijke conclusie over de klinische effectiviteit kon worden getrokken aan de hand van de huidige gepubliceerde resultaten in de literatuur. De reden waarom momenteel nog geen duidelijke uitspraak kan worden gedaan over de effectiviteit van deeltjesbestraling is voornamelijk door het gebrek aan methodologisch goed opgezette vergelijkende studies. Hierdoor is het moeilijk om verschillende behandelingen te vergelijken. Vaak zijn bijvoorbeeld de patientengroepen verschillend van samenstelling of is de onderzoeksopzet zodanig gekozen dat de selectie van de patientenpopulatie niet onwillekeurig heeft plaats gevonden waardoor vertekening van de resultaten kan ontstaan. Verder dient men in ogenschouw te houden dat een groot deel van het onderzoek naar deeltjestherapie is uitgevoerd in een fysisch laboratorium, los van een ziekenhuis of radiotherapeutisch instituut. De eisen aan de apparatuur binnen een fysisch laboratorium zijn over het algemeen minder streng vergeleken met de eisen waaraan de apparatuur moet voldoen binnen een medische instelling voor de behandeling van patiënten. Het is daarom aannemelijk te denken dat de resultaten uit het verleden, verkregen vanuit een fysisch laboratorium, gunstiger zouden zijn geweest indien deze waren verkregen binnen een medische instelling.

In ons literatuuronderzoek vonden we dat de klinische uitkomsten (overleving en het voorkomen van neveneffecten van de behandeling) van longkanker patiënten die behandeld waren met protonen therapie gelijk waren aan die van de moderne fotonenbestraling (b.v. stereotactische radiotherapie). Daarnaast lijkt een behandeling met koolstofionen minder neveneffecten te veroorzaken. Door de relatief grote onzekerheid rondom de beschikbare resultaten van eerder onderzoek naar de effectiviteit van deeltjesbestraling en daarbij de relatief hoge kosten voor deze behandeling, kan deeltjesbestraling (nog) niet als standaardbehandeling van longkanker worden inge- 
voerd. Echter, gezien het potentiële voordeel zoals is aangetoond in een aanzienlijk aantal planningsstudies die het overweldigende theoretische voordeel van protonen en koolstofionen beschrijven, is het gerechtvaardigd om verder toekomstig onderzoek naar de effectiviteit van deeltjesbestraling te verrichten. Vervolgens richt Hoofdstuk 8 zich op de verschillende aspecten vande kosten van deeltjestherapie.

Tot slot wordt in Hoofdstuk 9 beschreven, welke aspecten relevant kunnen zijn voor toekomstig onderzoek. Zo is het bijvoorbeeld zinvol om, in navolging op de tijdsfactoren zoals besproken in Hoofdstuk 2 en 3, meer in detail de impact van verschillende behandelfactoren te onderzoeken in de (chemo)radiotherapiebehandeling voor longkanker. Onlangs is een studie opgestart waarbij een meta-analyse van individuele patiënten zal worden uitgevoerd. Hierbij zullen de tijdsfactoren en het tijdstip van de start van een behandeling tot aan het einde van de RT (SER) nader worden onderzocht en zal tevens gekeken worden naar de invloed van andere behandelfactoren op de overleving van longkankerpatiënten, zoals de totale bestralingsdosis, de dosisintensiteit van de chemotherapie en de verschillende chemotherapie schema's.

Daarnaast, omdat er vele technologieën in ontwikkeling zijn, is het goed om te bepalen wat de kosteneffectiviteit is van deze behandelingen en hoe investeringen het meest adequaat gemaakt kunnen worden. Zo zijn we binnen een huidig kosteneffectiviteitsproject verschillende technologieën in de behandeling van longkanker aan het onderzoeken, zoals bijvoorbeeld stereotactische radiotherapie en deeltjestherapie. 

DANKWOORD 

Het boekje is af. Bij het schrijven van een dankwoord loop je het risico mensen vergeten, daarom wil ik vanaf deze plek graag iedereen bedanken die direct of indirect betrokken is geweest bij mijn promotietraject.

Allereerst wil ik van de gelegenheid gebruik maken om de patiënten te bedanken die bereid waren (relatief lange) vragenlijsten in te vullen. Zonder hen was een groot deel van dit promotieonderzoek niet mogelijk geweest.

Vervolgens wil ik mijn dank uitspreken naar Huub Backes. Beste Huub, als mijn direct leidinggevende wil ik je bedanken voor alle ruimte en mogelijkheden die je me de afgelopen jaren hebt gegeven om mijn kennis en vaardigheden m.b.t. wetenschappelijk onderzoek te ontwikkelen.

Prof. dr. Ph. Lambin, promotor. Beste Philippe, er is menig brainstorm over de structuur van mijn promotie traject gevoerd. Je ideeën waren (zijn) onuitputtelijk. Als alle ideeën die je hebt geopperd zouden zijn uitgevoerd, zou er voor minimaal nog 5 proefschriften materiaal op tafel liggen. $\mathrm{Na}$ ieder overleg volgde er een nieuwe ideeenlijst. Wil je ze nog zien? In ieder geval hartelijk dank voor het in mij gestelde vertrouwen en voor je enthousiaste en prettige begeleiding.

Dr. D.K.M. De Ruysscher, copromotor. Dirk, jij bent vanaf het begin betrokken bij vrijwel al mijn onderzoeksactiviteiten. Ik heb veel van je geleerd in de afgelopen jaren, en nog steeds, waar ik je erg dankbaar voor ben. Jouw enthousiasme voor wetenschappelijk onderzoek is "besmettelijk" en werkt (in ieder geval bij mij) motiverend. Je adequate reacties op mijn vragen en je snelle kritische commentaar op de door mij aangeleverde manuscripten heb ik als zeer prettig ervaren. Ondanks je drukke agenda wist je altijd tijd vrij te maken als ik weer eens met een "vraagje" je kamer binnenliep. Het risico bestond dan echter dat jouw ideeëngenerator werd geactiveerd waarna ik vervolgens je kamer verliet met nieuwe vragen en op zoek kon gaan naar de ontbrekende antwoorden. Dit kwam echter meestal ten goede van de kwaliteit van het onderzoek. M.b.t. het genereren van ideeën verwacht ik een hoge correlatie met je landgenoot zoals hierboven genoemd. Zouden personen met Belgische genen een significant hoger ideeën-gehalte hebben dan hun Noorderburen met een Nederlandse genstructuur? Inmiddels hebben we binnen MAASTRO een redelijke mix van Belgische en Nederlandse medewerkers. We zouden het kunnen onderzoeken......, ideetje?

Dr. L.J. Boersma, copromotor. Beste Liesbeth, jij hebt een grote rol gespeeld in de afronding van mijn promotieonderzoek. Op een constructieve wijze wist je altijd uiteenlopende ideeën terug te brengen tot de kern. Jouw kritische blik en je vermogen om op een prettige wijze een soms "warrig" geheel te ontrafelen en te structureren, 
waren erg waardevol. Hartelijk dank hiervoor! Ik vond het fijn je als copromotor te mogen betrekken bij dit proefschrift.

De leden van de beoordelingscommissie, te weten prof.dr. E.F. Wouters, Dr. A.M. Dingemans, prof.dr. J.A. Langendijk, prof.dr.Y. Lievens en prof.dr. J.L. Severens, wil ik bedanken voor alle tijd gespendeerd aan het lezen en beoordelen van dit proefschrift. Beste Hans, jammer dat je niet op de promotiedag zelf aanwezig kunt zijn. Ik had graag een zeer kritische vraag van je willen beantwoorden. Yolande, helaas ben jij ook verhinderd op 9 december. Ik hoop ooit je ervaringen van $500 \mathrm{~km}$ stappen in de woestijn van je te mogen horen. Succes ermee!

Een groot deel van mijn promotieonderzoek bestond uit systematisch literatuuronderzoek. Philippe, Dirk, Johan, Arnold en Isabelle wil ik bedanken voor de fijne samenwerking bij de totstandkoming van onze Cochrane review en de daaropvolgende publicaties elders.

Mijn wetenschappelijke carrière is min of meer gestart bij de Capgroep Epidemiologie. Pieter, Ruud, Arno, Sandra, Anouk en Brigitte, het is uiteindelijk gelukt een aantal mooie publicaties over kwaliteit van leven bij kankerpatiënten te publiceren. Twee daarvan maken deel uit van dit proefschrift. Bedankt voor de vele leermomenten en voor jullie bijdrage aan dit uiteindelijke resultaat.

Ruim 2 jaar geleden werd mijn interesse gewekt voor Medical Technology Assessment tijdens een gesprek met Hans Severens. Beste Hans, ik ben je erg dankbaar dat je me hebt gestimuleerd deze weg te kiezen. Manuela Joore wil ik hiernaast bedanken voor waardevolle ondersteuning bij de lopende MTA projecten.

Op deze plek wil ik mijn collegae bedanken. De interesse, zoals door velen getoond, hebben mij gesteund in de motivatie van dit werk. Daarnaast wil ik Harald, Ruud, Cary en Janneke bedanken voor het meedenken en het nalezen van stukken bij de totstandkoming van dit boekje. Tevens bedankt voor jullie hulp bij de lay-out.

Cary, we hebben al een lang gezamenlijk verleden binnen MAASTRO. Sinds 1987 ben je mijn collega. Je relativeringsvermogen is geweldig. Zowel als collega maar ook als vriendin is jouw steun erg waardevol geweest.

Verder wil ik alle DCM-medewerkers bedanken voor hun getoonde interesse en voor hun begrip voor mijn regelmatige afwezigheid in de laatste maanden. Vanaf nu ben ik er weer voor de volle $100 \%$.

Geert en Angela, jullie wil ik bedanken voor alle informatie en tips mbt alle voorbereiding en logistiek rondom een promotie. Rianne en Simone, graag wil ik jullie bedanken voor de secretariële ondersteuning en het versturen van de boekjes. 
Rinus en René, paranimfen. Het doorlopen van een promotietraject is misschien wel te vergelijken met het trainen voor een marathon. Het is tijdrovend en gaat vaak gepaard met ups en downs, maar als je uiteindelijk je doel bereikt voelt dat supergeweldig. Onze gezamenlijk duurlopen hebben indirect bijgedragen aan de totstandkoming van dit proefschrift. Als ik aangaf geen tijd te hebben (of gewoon geen zin had) om mijn sportschoenen aan te trekken wisten jullie me toch vaak te overtuigen dat 'het goed voor me was' een rondje met jullie mee te lopen. Jullie hadden gelijk. Rinus en René, bedankt voor alles in de afronding van mijn proefschrift. Trainen voor een marathon zat er dit jaar niet in. Volgend jaar staan de zaken er anders voor, dan loop ik met jullie mee!

Tot slot wil ik nog mijn vrienden en familie bedanken die gedurende dit promotietraject volop belangstelling hebben getoond.

Theo, Riny, Eef en Til, de laatste tijd was er weinig ruimte om gezellige avondjes te organiseren. Na 9 december gaan we er weer een paar inplannen!

Ine, ook al zien we elkaar niet zo vaak, ik kan me geen betere vriendin wensen. De momenten die we samen doorbrengen zijn zeer waardevol en hoop daarom dat er nog veel volgen in de toekomst.

Elfie en Theo, zus en broer. Elfie, zo nu en dan een weekendje samen relaxen doet wonderen, dat houden we erin! Theo, op 9 december ben je voor je werk in Ghana, heel ver weg. Jammer dat je er niet bij kunt zijn, we zullen je missen.

Speciale dank gaat uit naar mijn ouders. Lieve pap en mam, jullie hebben me altijd door dik en dun gesteund. Dankjewel voor deze altijd onvoorwaardelijke ondersteuning en belangstelling. Jullie stonden altijd voor ons klaar, niks was jullie teveel. In de afronding van mijn promotieonderzoek hebben jullie meegedacht over de stellingen en de kaft van mijn boekje. Ik heb dit als erg bijzonder ervaren.

Ralph, promotieonderzoek is 'vet' en het halen van de eindstreep 'kiks'. Een soortgelijk gevoel zal je ervaren als je over een tijdje je huidige studie kan afronden. Denk hierbij aan het spreekwoord 'zuinigheid met vlijt bouwt huizen als kastelen'.

Hey Mirthe en Lotte, ik hoop niet dat ik jullie mogelijke toekomstige interesse voor wetenschappelijk onderzoek heb doen dalen. Jullie hebben me herhaaldelijk laten weten later nooit mijn werk te willen doen. Maar..... het boekje is klaar. De binnenkant is van mij, de creatieve buitenkant van jullie. Mooi gedaan! Het zegt jullie waarschijnlijk nu nog niet zoveel, maar ik hoop dat jullie ooit de waarde van dit boekje duidelijk zal worden. Voor mij is een ding is duidelijk, jullie spelen een grote rol in mijn kwaliteit van leven. Daar is geen verder onderzoek voor nodig.

Lieve Raymond, ons boekje af. Zonder jou was het me nooit gelukt. Naast een intensieve verbouwing, hield jij de boel thuis draaiende en hebt me de ruimte gegeven om dit werk af te maken. Bedankt voor al je vertrouwen en geduld. Nu gaan we feesten op de goede afloop van ons werk. Proost! 

CURRICULUM VITAE 

Madelon Johannesma was born on April 22, 1965 in Sittard, The Netherlands. After completing secondary school at the former Albert Schweitzer comprehensive school (at current Graaf Huyn College) in Geleen, she started in 1984 with an apprenticeship for X-ray technician at the former Hospital "De Goddelijke Voorzienigheid" (at current Maasland Hospital) in Sittard, and graduated in 1987. In the same year she started a second apprenticeship for radiation technologist at the former "Radiotherapeutisch Instituut Limburg" (at current MAASTRO clinic) and graduated in 1989. From 1989 until 2003 she worked at MAASTRO as a radiation technologist with several additional functions such as Brachytherapy technologist and Coordinator Practice and Education.

Simultaneously, in 1999 she started studying Health education and Health promotion at the faculty of Health Sciences of Maastricht University, which resulted in a Master degree in January 2003. Subsequently she studied Epidemiology: Methods and Techniques of research in Health Sciences, also at the Maastricht University, resulting in a second Master degree in 2004. In 2005 she was registered as Epidemiologist-A in the national register.

From 2003 until current Madelon is working as a researcher within MAASTRO and in addition she holds a post as Head of the Data Centre since 2005. During this period she obtained within the two-year MPhil Health Sciences Research Master (HSRM) two certificates in Health Technology Assessment (General Principles of Economic Evaluations and Cost-effectiveness Modeling Methods) at the University of Maastricht.

Within her current position she is coordinator of two ongoing projects in the field of particle therapy, (1) cost-effectiveness of particle therapy and (2) in silico clinical trial on photons, protons and C-ions.

Part of her research activities during the last five years resulted in the present thesis. Madelon is married with Raymond Pijls and together they have two daughters, Mirthe and Lotte. 

PUBLICATIONS 


\section{PUBLICATIONS}

De Ruysscher D, Botterweck A, Dirx M, Pijls-Johannesma M, et al. Eligibility for concurrent chemotherapy and radiotherapy of locally advanced lung cancer patients: a prospective, population-based study. Ann Oncol, in press, 2008 Aug 20.

Pijls-Johannesma M, Pommier P and Lievens $\mathrm{Y}$. Cost-effectiveness of particle ther apy: Current evidence and future needs. Radiother Oncol, in press, 2008 Aug 14.

Brada M, De Ruysscher D and Pijls-Johannesma M. Evidence for Proton Therapy. J Clin Oncol 2008; 26(15):2592-2593.

Beijer S, Kempen GI, Pijls-Johannesma M, de Graeff A, Dagnelie PC. Determinants of overall quality of life in preterminal cancer patients. Int J Cancer 2008;123(1):2325.

Pijls-Johannesma M, Grutters JP, Lambin P, Ruysscher DD. Particle therapy in lung cancer: Where do we stand? Cancer Treat Rev 2008;34(3):259-67.

Pijls-Johannesma M, De Ruysscher D, Vansteenkiste J, Kester A, Rutten I, Lambin $P$. Timing of chest radiotherapy in patients with limited stage small cell lung cancer: a systematic review and meta-analysis of randomised controlled trials. Cancer Treat Rev 2007;33(5):461-73.

Lodge M, Pijls-Johannesma M, Stirk L, Munro AJ, De Ruysscher D, Jefferson T. A systematic literature review of the clinical and cost-effectiveness of hadron therapy in cancer. Radiother Oncol 2007;83(2):110-22.

Brada M, Pijls-Johannesma M, De Ruysscher D. Proton therapy in clinical practice: current clinical evidence. Journal of clinical oncology 2007;25(8):965-70.

Dagnelie P, Pijls-Johannesma M, Lambin P, Beijer S, De Ruysscher D, Kempen G. Impact of fatigue on overall quality of life in lung and breast cancer patients selected for high-dose radiotherapy. Annals of Oncology, 2007.

Pijls-Johannesma M, De Ruysscher D, Dekker AL, Lambin P. Potential advantages of proton and ion therapy in the treatment of cancer. Nederlands tijdschrift voor On cologie 2007; Augustus.

Perrier L, Combs S, Auberger T, Zucca L, Näslund I, Pijls-Johannesma M, et al. A Decision-Making Tool for a Costly Innovative Technology: the case of Carbon lon radiotherapy. Journal d"Economie Médicale 2007;25(7-8):367-380. 
De Ruysscher D, Dehing C, Bremer RH, Bentzen S, Koppe F, Pijls-Johannesma M, et al. Maximal neutropenia during chemotherapy and radiotherapy is significantly associated with the development of acute radiation-induced dysphagia in lung can cer patients. Annals of Oncology 2007.

Mul VE, van Geest AJ, Pijls-Johannesma M, Theys J, Verschueren TA, Jager JJ, et al. Radiation-induced bullous pemphigoid: a systematic review of an unusual radia tion side effect. Radiotherapy and oncology 2007;82(1):5-9.

Dagnelie PC, Pijls-Johannesma M, Pijpe A, Boumans BJ, Skrabanja AT, Lambin P, et al. Psychometric properties of the revised Piper Fatigue Scale in Dutch cancer patients were satisfactory. Journal of clinical epidemiology 2006;59(6):642-9.

De Ruysscher D, Bremer RH, Koppe F, Wanders S, van Haren E, Hochstenbag M, Geeraedts W, Pitz C, Simons J, ten Velde G, Dohmen J, Snoep G, Boersma L, Ver schueren T, van Baardwijk A, Dehing C, Pijls M, Minken A and Lambin P. Omission of elective node irradiation on basis of CT-scans in patients with LD small cell lung cancer: a phase II trial. Radiotherapy and oncology 2006;80(3):307-12.

De Ruysscher D, Pijls-Johannesma M, Bentzen SM, Minken A, Wanders R, Lut gens $L$, et al. Time between the first day of chemotherapy and the last day of chest radiation is the most important predictor of survival in limited-disease small-cell lung cancer. Journal of clinical oncology 2006;24(7):1057-63.

De Ruysscher D, Pijls-Johannesma M, Vansteenkiste J, Kester A, Rutten I, Lambin $P$. Systematic review and meta-analysis of randomised, controlled trials of the timing of chest radiotherapy in patients with limited-stage, small-cell lung cancer. Annals of oncology 2006;17(4):543-52.

Pijls-Johannesma MC, de Ruysscher DK, Dekker AL, Lambin P. [Protons and ions in the treatment of cancer; a systematic review of the literature]. Nederlands tijdschrift voor geneeskunde 2006;150(44):2435-41.

Pijls-Johannesma M, De Ruysscher D, Lambin P, Rutten I, Vansteenkiste JF. Early versus late chest radiotherapy for limited stage small cell lung cancer. Cochrane database of systematic reviews (Online) 2005(1):CD004700.

Pijls-Johannesma M, Pijpe A, Kempen GI, Lambin P, Dagnelie PC. Health related quality of life assessment instruments: a prospective study on preference and ac ceptability among cancer patients referred for radiotherapy. European journal of can cer (Oxford, England 2005;41(15):2250-6. 


\section{PUBLISHED ABSTRACTS}

Grutters J, Pijls-Johannesma M, De Ruysscher D, et al: Modeling the costeffectiveness of a new and expensive treatment modality in lung cancer: the case of particle therapy, ISPOR, Athens 2008

Peeters A, Grutters JPC, Pijls-johannesma M, et al: How costly is radiotherapy with particles? Cost analysis of external beam radiotherapy with carbon ions, protons and conventional photons, ISPOR, Athens, 2008

Peeters A, Joore MA, Pijls-Johannesma M, et al: Carbon ion therapy for prostate cancer seems cost-effective in the long term when quality of life is taken into account, but more evidence is still needed, ESTRO27. Göteborg, Sweden, 2008

Grutters J, Pijls-Johannesma M, Joore M, et al: Particle therapy appears to be a cost-effective treatment modality as opposed to conventional 3D radiotherapy and stereotactic body radiotherapy for inoperable stage I non-small cell lung cancer pa tients, but more evidence is needed, ESTRO27. Götebrog, Sweden, 2008

Pijls-Johannesma M, Dekker A, Qamhiyeh S, et al: International, multicentric In Silico clinical trial in lung, prostate and head\&neck cancer, comparing photons, pro tons and C-ion therapy treatment: a multicentric planning study based on a reference dataset of patients: Evaluation of feasibility, ESTRO27. Göteborg, Sweden, 2008

Pijls-Johannesma M, Mastrigt Gv, Ruysscher DD, et al: A systematic review of the methodology of phase I radiation dose escalation trials in patients with lung cancer, ESTRO27. Góteborg, Sweden, 2008

Lambin Ph, Mastrigt Gv, Ruysscher DD, Pijls-Johannesma M, et al: Preliminary results of a Cochrane review on the adjuvant effects of EPO on radiation therapy in patients with Head and Neck cancer, ESTRO 27. Göteborg, Sweden, 2008

Lutgens LCHW, Zee Jvd, Haas-Kock DFMd, Mastrigt Gv, Pijls-Johannesma et al: The role of hyperthermia in the treatment of locally advanced cervix carcinoma with radiotherapy: a systematic review. Preliminary results on behalf of the Cochrane Gy naecological Cancer Review Group, ESTRO27. Göteborg, Sweden, 2008

Haas-Kock DFMd, Buijsen J, Mastrigt GAv, Pijls-Johannesma et al: The role of hy perthermia in pre-operative radiotherapy in rectal cancer: a systematic review, ESTRO27. Göteborg, Sweden, 2008 
Engelsman M, Rasch C, Pijls-Johannesma M, et al: Preliminary results of an inter national multi-modality in-silico trial for lung cancer, ESTRO27. Göteborg, Sweden, 2008

Houben R, Eskandarian M, Pijls-Johannesma M, et al: Measurement of common toxicity of patients treated with radiotherapy for prostate carcinoma: Psychometric properties of self-report questionnaires measuring radiation cystitis, radiation proctitis and erectile function, ESTRO 27. Göteborg, Sweden, 2008

Qamhiyeh S, Pijls-Johannesma M, Baumert BG, et al: In Silico clinical trial in lung, prostate and head\&neck cancer, comparing photons, protons and C-ion therapy treatment: a multicentric planning study based on a reference dataset of patients: Evaluation of feasibility, PTCOG. Jacksonville, Florida, 2008

Grutters J, Peeters A, Pijls-Johannesma et al: Modeling the cost-effectiveness of proton and carbon-ion treatment for lung and prostate cancer: do we need more evi dence?, PTCOG 47. Jacksonville, Florida, 2008, pp 63(P21)

Pijls-Johannesma M, Lambin P, Ruysscher DD: Protons and C-ions for the treat ment of non-small cell lung cancer (NSCLC): What is published the evidence?, IASLC. Seoul, 2007

Pijls-Johannesma M, Ruysscher DD, Lambin P: Cost-effectiveness of particle therapy: current evidence and future needs, ECCO14. Barcelona, 2007

Simons P, Pijls-Johannesma M, Kempen GIJM, et al: What is the best method to evaluate the Quality of Life during curative radiotherapy? A comparison between the SEIQoL-DW and the EORTC-QLQ questionnaires, ESTRO25. Leipzig, 2006

Ruysscher DD, Dehing C, Bentzen SM, Pijls-Johannesma, et al: The maximal neu tropenia during concurrent chemotherapy and radiotherapy is the strongest predictor for the development of acute radiation-induced esophagitis in lung cancer patients: involvement of bone marrow epithelial stem cells?, ESTRO25. Leipzig, 2006

Pijls-Johannesma M, Seghers K, Houben R, et al: The of QoL of patients with lung cancer treated with high dose radiotherapy or concurrent chemo-radiation only shows a temporary, reversible decline: a longitudinal data analysis of symptoms, function ing and esophageal toxicity to global QoL, ESTRO25. Leipzig, 2006

Pijls-Johannesma M, Lodge M, Stirk L, et al: A systematic literature review of the clinical and cost effectiveness of hadron therapy in cancer, ESTRO25. Leipzig, 2006 
Dagnelie PC, Pijls-Johannesma M, Lambin P, et al: Determinants of Overall Quality of Life in Cancer Patients Selected for High-Dose Radiotherapy ESTRO25. Leipzig, 2006

Macias V, Pijls-Johannesma M, Marcos-Pascua P, et al: High Dose Hypofraction ated 3D Conformal Radiotherapy In Localised Prostate Cancer: Preliminary re sults, ESTRO25. Leipzig, 2006

Feldman-Stewart D, Capirci C, Abacioglu U, Pijls-Johannesma M, et al: Information Needs of Early-Stage Prostate Cancer Patients: A comparison of seven European Countries and Canada, ESTRO25. Leipzig, 2006

Feldman-Stewart D, Capirci C, Brennenstuhl S, Pijls-Johannesma M, et al: Decision Making Needs of Early-Stage Prostate Cancer Patients: A comparison of seven European Countries and Canada, 28th Annual Meeting of the Society for Medical Decision Making. Cambridge, Society for Medical Decision Making, Boston, 2006

Pijls-Johannesma M, Ruysscher DD, Rutten I, et al: Early versus late chest radio therapy for limited stage small cell lung cancer: a systematic review and metaanalysis, IASLC. Barcelona, 2005

Pijls-Johannesma M, Ruysscher DD, Rutten I, et al: Early versus late chest radio therapy for limited stage small cell lung cancer: A systematic Review and Metaanalysis, ESTRO22. Amsterdam, 2004

Pijls-Johannesma M, Kempen GIJM, Dagnelie PC: Fatigue in lung- and breast can cer patients prior to radiotherapy ESTRO21. Praha, 2003

Pijls-Johannesma M, Kempen GIJM, Skrabanja ATP, et al: Fatigue in lung- and breast cancer patients prior to radiotherapy EORTC. Tübingen, Germany, 2003

Pijls M, Willems D, Braeken V, et al: Measurement of xerostomy in head and neck cancer patients ESTRO21. Geneve, 2003

Pijls M, Willems D, Braeken V, et al: Comparison of three questionnaires to quantify xerostomy in head and neck cancer patients treated by radiotherapy, ESTRO20.

Praha, 2002 